\title{
Allosteric Signal Amplification Sensing Using a Bisthiourea-Binaphthyl-Polythiophene Conjugate: A Positive Homotropic Allosterim Case
}

\author{
Tomoaki Tsuchiya ${ }^{\dagger}$ and Gaku Fukuhara $*,+,+$ \\ ${ }^{\dagger}$ Department of Chemistry, Tokyo Institute of Technology, 2-12-1 Ookayama, Meguro-ku, Tokyo 152-8551, \\ Japan \\ $₫$ JST, PRESTO, 4-1-8 Honcho, Kawaguchi, Saitama 332-0012, Japan \\ E-mail: gaku@chem.titech.ac.jp
}

Table of Contents

page

Theoretical Calculations of Monomer Analogs

Synthesis and Characterization of Bisthiourea-Binaphthyl-Polythiophene S32-44

Conjugate

Summary of Titration Data and Curve Fittings for M and P Chemosensors

Comparison Titration Curves

Waveform Separation Spectra

Theoretical Calculations of Oligomer Analogs

Comparison ${ }^{1} \mathrm{H}$ NMR Spectra 


\section{Theoretical Calculations of Monomer Analogs}

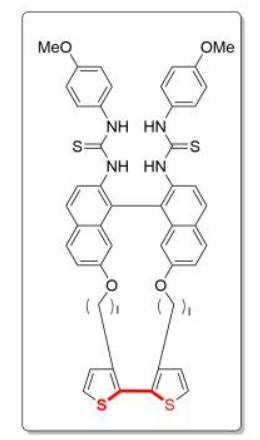

(e)
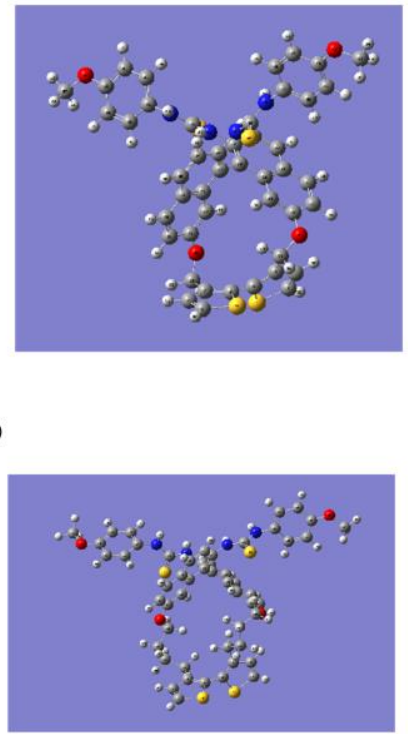

(c)
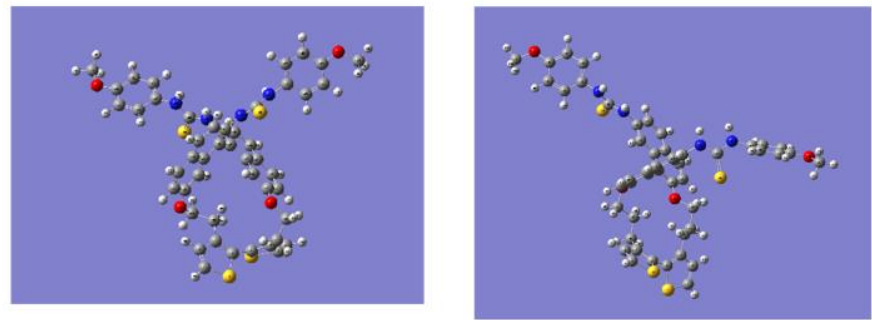

(f)

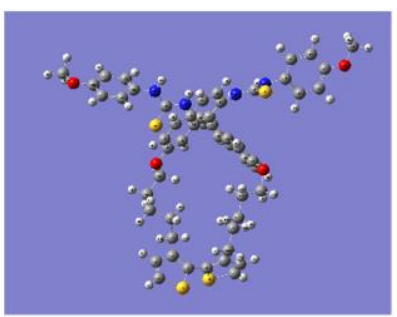

Figure S1. (a) Chemical structure of the monomer (M) analog; the oxyethylene chains and the chloro-groups were omitted for simplified calculations. Optimized structures of the $\mathbf{M}$ analogs by Gaussian 16 calculation (HF/6-31G); (b) $l=1$, (c) $l=2$, (d) $l=3$, (e) $l=4$, and (f) $l=5$. The number of imaginary frequencies was not involved during the calculation due to a high running cost.

(a)

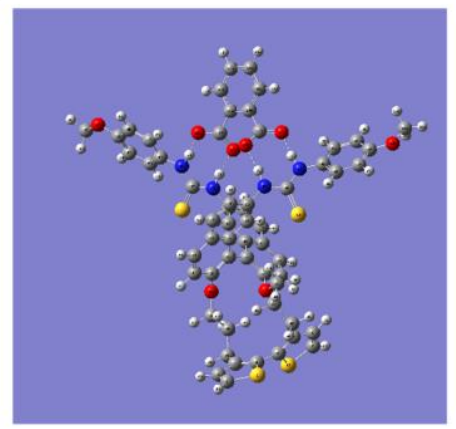

(b)

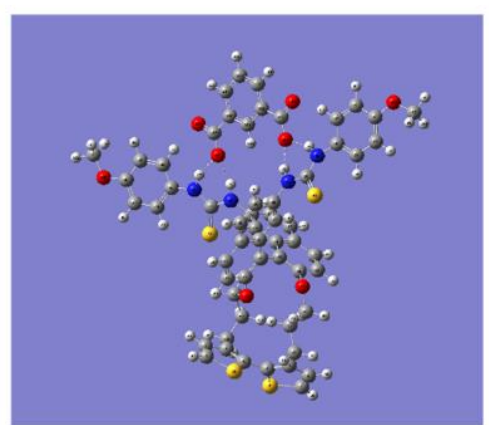

(c)

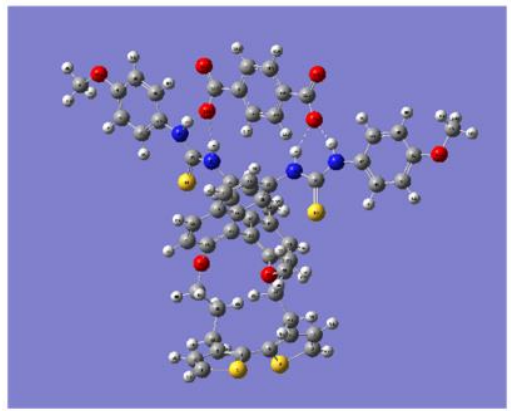

Figure S2. Optimized structures of the $\mathbf{M}$ analog $(l=3)$ in the presence of (a) phthalate, (b) isophthalate, and (c) terephthalate by Gaussian 16 calculation (HF/6-31G); counter cation was omitted for clarity. The number of imaginary frequencies was not involved during the calculation due to a high running cost. 
Table S1. Dihedral Angles Obtained from M Analogs Optimized using Gaussian 16

Calculations $^{a}$

\begin{tabular}{ccccc}
\hline \multirow{2}{*}{$\begin{array}{c}\text { Number of } \\
\text { methylene chains }(l)\end{array}$} & Dihedral angle $/^{\circ} b$ & \multicolumn{3}{c}{ Dihedral angle of $\mathbf{M}$ analog $(l=3)$ with guests ${ }^{\mathbf{o}}$} \\
\cline { 3 - 5 } & 119.82 & phthalate $^{c}$ & isophthalate $^{c}$ & terephthalate $^{c}$ \\
\hline 1 & 99.93 & - & - & - \\
3 & 64.84 & - & - & - \\
4 & 87.19 & 121.99 & 122.38 & 123.06 \\
5 & 92.71 & - & - & - \\
& & - & - & -
\end{tabular}

${ }^{a}$ Basis set: HF/6-31G. ${ }^{b} \mathrm{~S}-\mathrm{C}-\mathrm{C}-\mathrm{S}$ bond in the bithiophene unit (red color in Figure S1a). ${ }^{c}$ Counter cation was omitted for simplicity.

\section{Summary of Structure Optimization}

$\mathbf{M}$ analogs $;=1$

Z-matrices

\begin{tabular}{|c|c|c|c|c|c|c|c|c|c|c|}
\hline $\mathrm{C}$ & 0 & 1 & 0 & 1 & 0 & 1 & 0 & 0 & 0 & \# \\
\hline $\mathrm{C}$ & 1.339259 & 1 & 0 & 1 & 0 & 1 & 1 & 0 & 0 & \# \\
\hline $\mathrm{C}$ & 1.446127 & 1 & 114.3455 & 1 & 0 & 1 & 2 & 1 & 0 & \# \\
\hline $\mathrm{C}$ & 1.353085 & 1 & 113.2685 & 1 & 0.515836 & 1 & 3 & 2 & 1 & \# \\
\hline$S$ & 1.78043 & 1 & 111.8012 & 1 & -0.8271 & 1 & 1 & 2 & 3 & \# \\
\hline $\mathrm{C}$ & 1.459283 & 1 & 129.8958 & 1 & 173.6196 & 1 & 4 & 3 & 2 & \# \\
\hline $\mathrm{C}$ & 1.355023 & 1 & 131.2263 & 1 & 131.9819 & 1 & 6 & 4 & 3 & \# \\
\hline $\mathrm{C}$ & 1.4468 & 1 & 113.0718 & 1 & 175.022 & 1 & 7 & 6 & 4 & \# \\
\hline $\mathrm{C}$ & 1.339652 & 1 & 114.829 & 1 & 0.287816 & 1 & 8 & 7 & 6 & \# \\
\hline$S$ & 1.778078 & 1 & 111.3254 & 1 & -0.46326 & 1 & 9 & 8 & 7 & \# \\
\hline $\mathrm{C}$ & 3.636899 & 1 & 135.6264 & 1 & -121.244 & 1 & 2 & 1 & 5 & \# \\
\hline $\mathrm{C}$ & 1.35802 & 1 & 129.8224 & 1 & 19.62462 & 1 & 11 & 2 & 1 & \# \\
\hline $\mathrm{C}$ & 1.419237 & 1 & 120.9115 & 1 & 83.71714 & 1 & 12 & 11 & 2 & \# \\
\hline $\mathrm{C}$ & 1.411249 & 1 & 119.1354 & 1 & 3.811306 & 1 & 13 & 12 & 11 & \# \\
\hline $\mathrm{C}$ & 1.42246 & 1 & 118.8322 & 1 & -2.32486 & 1 & 14 & 13 & 12 & \# \\
\hline $\mathrm{C}$ & 1.360059 & 1 & 120.1144 & 1 & -2.2698 & 1 & 15 & 14 & 13 & \# \\
\hline $\mathrm{C}$ & 1.414813 & 1 & 121.6967 & 1 & -175.266 & 1 & 13 & 12 & 11 & \# \\
\hline $\mathrm{C}$ & 1.357824 & 1 & 121.2368 & 1 & 176.2126 & 1 & 17 & 13 & 12 & \# \\
\hline $\mathrm{C}$ & 1.416483 & 1 & 119.9142 & 1 & 1.106028 & 1 & 18 & 17 & 13 & \# \\
\hline $\mathrm{C}$ & 1.379657 & 1 & 120.8209 & 1 & 3.544393 & 1 & 19 & 18 & 17 & \# \\
\hline $\mathrm{C}$ & 3.036123 & 1 & 108.6298 & 1 & -63.2344 & 1 & 7 & 6 & 4 & \# \\
\hline $\mathrm{C}$ & 1.362531 & 1 & 130.1843 & 1 & -0.80849 & 1 & 21 & 7 & 6 & \# \\
\hline $\mathrm{C}$ & 1.416559 & 1 & 120.4994 & 1 & -111.332 & 1 & 22 & 21 & 7 & \# \\
\hline
\end{tabular}




\begin{tabular}{|c|c|c|c|c|c|c|c|c|c|c|}
\hline $\mathrm{C}$ & 1.412956 & 1 & 118.7795 & 1 & -1.53673 & 1 & 23 & 22 & 21 & \# \\
\hline $\mathrm{C}$ & 1.415124 & 1 & 118.9857 & 1 & -2.87954 & 1 & 24 & 23 & 22 & \# \\
\hline $\mathrm{C}$ & 1.361889 & 1 & 121.174 & 1 & 3.799277 & 1 & 25 & 24 & 23 & \# \\
\hline $\mathrm{C}$ & 1.433999 & 1 & 121.7965 & 1 & 173.9754 & 1 & 23 & 22 & 21 & \# \\
\hline $\mathrm{C}$ & 1.376683 & 1 & 119.1085 & 1 & -171.1 & 1 & 27 & 23 & 22 & \# \\
\hline $\mathrm{C}$ & 1.418725 & 1 & 121.0445 & 1 & -6.29113 & 1 & 28 & 27 & 23 & \# \\
\hline $\mathrm{C}$ & 1.35613 & 1 & 119.9636 & 1 & 3.487835 & 1 & 29 & 28 & 27 & \# \\
\hline $\mathrm{O}$ & 1.382546 & 1 & 123.4176 & 1 & -175.255 & 1 & 16 & 15 & 14 & \# \\
\hline $\mathrm{O}$ & 1.377649 & 1 & 53.92899 & 1 & 95.85357 & 1 & 21 & 7 & 6 & \# \\
\hline $\mathrm{N}$ & 1.421233 & 1 & 120.3889 & 1 & 178.7903 & 1 & 19 & 18 & 17 & \# \\
\hline $\mathrm{N}$ & 1.421958 & 1 & 119.1129 & 1 & 178.2484 & 1 & 28 & 27 & 23 & \# \\
\hline $\mathrm{C}$ & 1.359089 & 1 & 129.9021 & 1 & 43.27317 & 1 & 33 & 19 & 18 & \# \\
\hline $\mathrm{C}$ & 1.358523 & 1 & 128.6372 & 1 & -134.713 & 1 & 34 & 28 & 27 & \# \\
\hline $\mathrm{N}$ & 1.349887 & 1 & 112.315 & 1 & 178.9712 & 1 & 35 & 33 & 19 & \# \\
\hline S & 1.73227 & 1 & 125.4602 & 1 & 0.292015 & 1 & 35 & 33 & 19 & \# \\
\hline $\mathrm{N}$ & 1.348502 & 1 & 112.6359 & 1 & 178.8046 & 1 & 36 & 34 & 28 & \# \\
\hline S & 1.732856 & 1 & 124.8191 & 1 & 0.029086 & 1 & 36 & 34 & 28 & \# \\
\hline $\mathrm{C}$ & 1.430564 & 1 & 126.4094 & 1 & -177.586 & 1 & 37 & 35 & 33 & \# \\
\hline $\mathrm{C}$ & 1.430751 & 1 & 126.3727 & 1 & -177.485 & 1 & 39 & 36 & 34 & \# \\
\hline $\mathrm{C}$ & 1.381456 & 1 & 120.4069 & 1 & 82.96244 & 1 & 41 & 37 & 35 & \# \\
\hline $\mathrm{C}$ & 1.388773 & 1 & 120.4843 & 1 & 177.6549 & 1 & 43 & 41 & 37 & \# \\
\hline $\mathrm{C}$ & 1.386593 & 1 & 119.4887 & 1 & -0.22573 & 1 & 44 & 43 & 41 & \# \\
\hline $\mathrm{C}$ & 1.390609 & 1 & 120.2298 & 1 & 0.123699 & 1 & 45 & 44 & 43 & \# \\
\hline $\mathrm{C}$ & 1.379729 & 1 & 119.8107 & 1 & 0.200479 & 1 & 46 & 45 & 44 & \# \\
\hline $\mathrm{C}$ & 1.390061 & 1 & 119.878 & 1 & -98.9912 & 1 & 42 & 39 & 36 & \# \\
\hline $\mathrm{C}$ & 1.379689 & 1 & 120.2759 & 1 & -177.375 & 1 & 48 & 42 & 39 & \# \\
\hline $\mathrm{C}$ & 1.39068 & 1 & 119.8133 & 1 & -0.4054 & 1 & 49 & 48 & 42 & \# \\
\hline $\mathrm{C}$ & 1.38657 & 1 & 120.2345 & 1 & 0.185985 & 1 & 50 & 49 & 48 & \# \\
\hline $\mathrm{C}$ & 1.381431 & 1 & 120.3672 & 1 & 83.33097 & 1 & 42 & 39 & 36 & \# \\
\hline $\mathrm{C}$ & 1.441479 & 1 & 121.9614 & 1 & 44.00464 & 1 & 31 & 16 & 15 & \# \\
\hline $\mathrm{C}$ & 1.440293 & 1 & 122.606 & 1 & 26.19886 & 1 & 32 & 21 & 7 & \# \\
\hline $\mathrm{H}$ & 1.066498 & 1 & 128.3014 & 1 & 178.9668 & 1 & 1 & 2 & 3 & \# \\
\hline $\mathrm{H}$ & 1.067794 & 1 & 123.434 & 1 & 179.4889 & 1 & 2 & 1 & 5 & \# \\
\hline $\mathrm{H}$ & 1.071123 & 1 & 122.3714 & 1 & -179.856 & 1 & 8 & 7 & 6 & \# \\
\hline $\mathrm{H}$ & 1.066382 & 1 & 128.4864 & 1 & 179.1279 & 1 & 9 & 8 & 7 & \# \\
\hline $\mathrm{H}$ & 1.070888 & 1 & 73.63112 & 1 & -98.5185 & 1 & 11 & 2 & 1 & \# \\
\hline $\mathrm{H}$ & 1.07338 & 1 & 120.266 & 1 & -94.4755 & 1 & 12 & 11 & 2 & \# \\
\hline $\mathrm{H}$ & 1.073237 & 1 & 119.0164 & 1 & -2.59994 & 1 & 17 & 13 & 12 & \# \\
\hline
\end{tabular}




\begin{tabular}{|c|c|c|c|c|c|c|c|c|c|}
\hline $\mathrm{H}$ & 1.066543 & 1 & 120.7862 & 1 & -177.846 & 1 & 18 & 17 & 13 \\
\hline $\mathrm{H}$ & 1.073523 & 1 & 118.8043 & 1 & -177.662 & 1 & 25 & 24 & 23 \\
\hline $\mathrm{H}$ & 1.070056 & 1 & 120.1796 & 1 & 179.2571 & 1 & 26 & 25 & 24 \\
\hline $\mathrm{H}$ & 1.067412 & 1 & 119.0627 & 1 & -177.575 & 1 & 29 & 28 & 27 \\
\hline $\mathrm{H}$ & 1.07327 & 1 & 119.8909 & 1 & 179.9198 & 1 & 30 & 29 & 28 \\
\hline $\mathrm{H}$ & 0.995453 & 1 & 113.5534 & 1 & -150.919 & 1 & 33 & 19 & 18 \\
\hline $\mathrm{H}$ & 0.995459 & 1 & 114.2704 & 1 & 31.03235 & 1 & 34 & 28 & 27 \\
\hline $\mathrm{H}$ & 0.996212 & 1 & 118.0754 & 1 & 3.224353 & 1 & 37 & 35 & 33 \\
\hline $\mathrm{H}$ & 0.99625 & 1 & 118.0627 & 1 & 3.033672 & 1 & 39 & 36 & 34 \\
\hline $\mathrm{H}$ & 1.071805 & 1 & 119.5442 & 1 & -3.28309 & 1 & 43 & 41 & 37 \\
\hline $\mathrm{H}$ & 1.07026 & 1 & 119.3326 & 1 & 179.5078 & 1 & 44 & 43 & 41 \\
\hline $\mathrm{H}$ & 1.070487 & 1 & 118.6194 & 1 & 179.623 & 1 & 46 & 45 & 44 \\
\hline $\mathrm{H}$ & 1.072309 & 1 & 120.172 & 1 & 178.8901 & 1 & 47 & 46 & 45 \\
\hline $\mathrm{H}$ & 1.072293 & 1 & 119.5382 & 1 & 3.292048 & 1 & 48 & 42 & 39 \\
\hline $\mathrm{H}$ & 1.070485 & 1 & 121.5679 & 1 & -179.834 & 1 & 49 & 48 & 42 \\
\hline $\mathrm{H}$ & 1.070245 & 1 & 121.1871 & 1 & -179.618 & 1 & 51 & 50 & 49 \\
\hline $\mathrm{H}$ & 1.07184 & 1 & 119.5587 & 1 & -3.23095 & 1 & 52 & 42 & 39 \\
\hline $\mathrm{H}$ & 1.078893 & 1 & 110.8415 & 1 & -51.1697 & 1 & 53 & 31 & 16 \\
\hline $\mathrm{H}$ & 1.078435 & 1 & 103.5724 & 1 & -167.075 & 1 & 53 & 31 & 16 \\
\hline $\mathrm{H}$ & 1.075407 & 1 & 103.1634 & 1 & -174.121 & 1 & 54 & 32 & 21 \\
\hline $\mathrm{H}$ & 1.081863 & 1 & 109.4796 & 1 & 71.11311 & 1 & 54 & 32 & 21 \\
\hline $\mathrm{C}$ & 2.445545 & 1 & 145.5546 & 1 & -177.346 & 1 & 50 & 49 & 48 \\
\hline $\mathrm{H}$ & 1.075988 & 1 & 133.9329 & 1 & -2.44389 & 1 & 83 & 50 & 49 \\
\hline $\mathrm{H}$ & 1.082036 & 1 & 95.11044 & 1 & 121.3936 & 1 & 83 & 50 & 49 \\
\hline $\mathrm{H}$ & 1.082214 & 1 & 96.55796 & 1 & -127.948 & 1 & 83 & 50 & 49 \\
\hline $\mathrm{C}$ & 2.445569 & 1 & 94.19387 & 1 & 178.625 & 1 & 45 & 44 & 43 \\
\hline $\mathrm{H}$ & 1.07599 & 1 & 133.9322 & 1 & 179.6783 & 1 & 87 & 45 & 44 \\
\hline $\mathrm{H}$ & 1.082043 & 1 & 95.06385 & 1 & -56.542 & 1 & 87 & 45 & 44 \\
\hline $\mathrm{H}$ & 1.082233 & 1 & 96.60973 & 1 & 54.11599 & 1 & 87 & 45 & 44 \\
\hline $\mathrm{O}$ & 1.369984 & 1 & 115.8165 & 1 & -179.993 & 1 & 50 & 49 & 48 \\
\hline $\mathrm{O}$ & 1.370039 & 1 & 123.9499 & 1 & -179.675 & 1 & 45 & 44 & 43 \\
\hline $\mathrm{H}$ & 1.06893 & 1 & 118.4369 & 1 & 65.50371 & 1 & 22 & 21 & 7 \\
\hline $\mathrm{H}$ & 1.068851 & 1 & 119.37 & 1 & 179.9649 & 1 & 15 & 14 & 13 \\
\hline & 0 & 0 & 0 & 0 & 0 & 0 & 0 & 0 & 0 \\
\hline
\end{tabular}

Total energy $=-3869.133040$ Hartree 
$\mathbf{M}$ analogs; $l=2$

Z-matrices

\begin{tabular}{|c|c|c|c|c|c|c|c|c|c|c|}
\hline $\mathrm{C}$ & 0 & 1 & 0 & 1 & 0 & 1 & 0 & 0 & 0 & \# \\
\hline $\mathrm{C}$ & 1.340161 & 1 & 0 & 1 & 0 & 1 & 1 & 0 & 0 & \# \\
\hline $\mathrm{C}$ & 1.45014 & 1 & 114.2711 & 1 & 0 & 1 & 2 & 1 & 0 & $\#$ \\
\hline $\mathrm{C}$ & 1.351911 & 1 & 113.0198 & 1 & -0.4242 & 1 & 3 & 2 & 1 & \# \\
\hline S & 1.782132 & 1 & 112.0103 & 1 & 0.047378 & 1 & 1 & 2 & 3 & \# \\
\hline $\mathrm{C}$ & 1.461295 & 1 & 129.6281 & 1 & 178.8729 & 1 & 4 & 3 & 2 & \# \\
\hline $\mathrm{C}$ & 1.351324 & 1 & 130.0028 & 1 & 105.6835 & 1 & 6 & 4 & 3 & \# \\
\hline $\mathrm{C}$ & 1.450628 & 1 & 113.0512 & 1 & 175.9455 & 1 & 7 & 6 & 4 & \# \\
\hline $\mathrm{C}$ & 1.339849 & 1 & 114.6139 & 1 & 0.67018 & 1 & 8 & 7 & 6 & $\#$ \\
\hline$S$ & 1.780807 & 1 & 111.6488 & 1 & -0.62997 & 1 & 9 & 8 & 7 & \# \\
\hline $\mathrm{C}$ & 4.463037 & 1 & 138.7672 & 1 & -132.878 & 1 & 2 & 1 & 5 & $\#$ \\
\hline $\mathrm{C}$ & 1.357485 & 1 & 141.1784 & 1 & 60.19414 & 1 & 11 & 2 & 1 & \# \\
\hline $\mathrm{C}$ & 1.421565 & 1 & 121.106 & 1 & 77.82352 & 1 & 12 & 11 & 2 & $\#$ \\
\hline $\mathrm{C}$ & 1.409486 & 1 & 118.8544 & 1 & 2.595609 & 1 & 13 & 12 & 11 & \# \\
\hline $\mathrm{C}$ & 1.42142 & 1 & 119.0771 & 1 & -0.36056 & 1 & 14 & 13 & 12 & \# \\
\hline $\mathrm{C}$ & 1.361392 & 1 & 120.2856 & 1 & -3.16313 & 1 & 15 & 14 & 13 & $\#$ \\
\hline $\mathrm{C}$ & 1.414241 & 1 & 122.1319 & 1 & -176.154 & 1 & 13 & 12 & 11 & $\#$ \\
\hline $\mathrm{C}$ & 1.359466 & 1 & 121.3238 & 1 & 175.7628 & 1 & 17 & 13 & 12 & \# \\
\hline $\mathrm{C}$ & 1.415878 & 1 & 119.8687 & 1 & -0.00408 & 1 & 18 & 17 & 13 & \# \\
\hline $\mathrm{C}$ & 1.378113 & 1 & 120.7275 & 1 & 4.023735 & 1 & 19 & 18 & 17 & $\#$ \\
\hline $\mathrm{C}$ & 3.725141 & 1 & 104.9092 & 1 & -75.7719 & 1 & 7 & 6 & 4 & $\#$ \\
\hline $\mathrm{C}$ & 1.36706 & 1 & 139.3414 & 1 & 22.81924 & 1 & 21 & 7 & 6 & \# \\
\hline $\mathrm{C}$ & 1.41372 & 1 & 120.7941 & 1 & -107.042 & 1 & 22 & 21 & 7 & \# \\
\hline $\mathrm{C}$ & 1.413284 & 1 & 118.8572 & 1 & -2.77106 & 1 & 23 & 22 & 21 & $\#$ \\
\hline $\mathrm{C}$ & 1.413513 & 1 & 118.7608 & 1 & -0.14619 & 1 & 24 & 23 & 22 & $\#$ \\
\hline $\mathrm{C}$ & 1.36404 & 1 & 121.5438 & 1 & 2.201428 & 1 & 25 & 24 & 23 & $\#$ \\
\hline $\mathrm{C}$ & 1.436578 & 1 & 121.8638 & 1 & 173.1815 & 1 & 23 & 22 & 21 & \# \\
\hline $\mathrm{C}$ & 1.373004 & 1 & 119.0873 & 1 & -173.786 & 1 & 27 & 23 & 22 & $\#$ \\
\hline $\mathrm{C}$ & 1.419092 & 1 & 121.3137 & 1 & -5.06943 & 1 & 28 & 27 & 23 & \# \\
\hline $\mathrm{C}$ & 1.355503 & 1 & 119.8963 & 1 & 3.870579 & 1 & 29 & 28 & 27 & $\#$ \\
\hline $\mathrm{O}$ & 1.372209 & 1 & 124.4031 & 1 & -174.511 & 1 & 16 & 15 & 14 & \# \\
\hline $\mathrm{O}$ & 1.372239 & 1 & 56.7726 & 1 & 114.5316 & 1 & 21 & 7 & 6 & $\#$ \\
\hline $\mathrm{N}$ & 1.421739 & 1 & 120.8786 & 1 & 179.9397 & 1 & 19 & 18 & 17 & \# \\
\hline $\mathrm{N}$ & 1.423981 & 1 & 119.3102 & 1 & 177.8261 & 1 & 28 & 27 & 23 & $\#$ \\
\hline $\mathrm{C}$ & 1.358713 & 1 & 130.2024 & 1 & 41.83422 & 1 & 33 & 19 & 18 & $\#$ \\
\hline $\mathrm{C}$ & 1.357459 & 1 & 127.4861 & 1 & -126.994 & 1 & 34 & 28 & 27 & \# \\
\hline
\end{tabular}




\begin{tabular}{|c|c|c|c|c|c|c|c|c|c|c|}
\hline $\mathrm{N}$ & 1.350479 & 1 & 112.3078 & 1 & 179.2116 & 1 & 35 & 33 & 19 & \# \\
\hline S & 1.732095 & 1 & 125.5518 & 1 & 0.570321 & 1 & 35 & 33 & 19 & \# \\
\hline $\mathrm{N}$ & 1.347498 & 1 & 113.0085 & 1 & 178.287 & 1 & 36 & 34 & 28 & \# \\
\hline S & 1.733839 & 1 & 124.2776 & 1 & -0.72282 & 1 & 36 & 34 & 28 & \# \\
\hline $\mathrm{C}$ & 1.430523 & 1 & 126.3968 & 1 & -177.684 & 1 & 37 & 35 & 33 & \# \\
\hline $\mathrm{C}$ & 1.431088 & 1 & 126.2741 & 1 & -177.131 & 1 & 39 & 36 & 34 & \# \\
\hline $\mathrm{C}$ & 1.381356 & 1 & 120.3753 & 1 & 84.53977 & 1 & 41 & 37 & 35 & \# \\
\hline $\mathrm{C}$ & 1.38891 & 1 & 120.5008 & 1 & 177.6196 & 1 & 43 & 41 & 37 & $\#$ \\
\hline $\mathrm{C}$ & 1.386512 & 1 & 119.4767 & 1 & -0.17667 & 1 & 44 & 43 & 41 & $\#$ \\
\hline $\mathrm{C}$ & 1.390707 & 1 & 120.2296 & 1 & 0.109382 & 1 & 45 & 44 & 43 & \# \\
\hline $\mathrm{C}$ & 1.379604 & 1 & 119.8167 & 1 & 0.170806 & 1 & 46 & 45 & 44 & \# \\
\hline $\mathrm{C}$ & 1.39071 & 1 & 120.0159 & 1 & 89.2997 & 1 & 42 & 39 & 36 & $\#$ \\
\hline $\mathrm{C}$ & 1.378985 & 1 & 120.2086 & 1 & 177.5079 & 1 & 48 & 42 & 39 & \# \\
\hline $\mathrm{C}$ & 1.391288 & 1 & 119.8693 & 1 & 0.121034 & 1 & 49 & 48 & 42 & \# \\
\hline $\mathrm{C}$ & 1.386085 & 1 & 120.2507 & 1 & -0.01445 & 1 & 50 & 49 & 48 & \# \\
\hline $\mathrm{C}$ & 1.380761 & 1 & 120.2111 & 1 & -92.9815 & 1 & 42 & 39 & 36 & \# \\
\hline $\mathrm{C}$ & 1.443797 & 1 & 122.6245 & 1 & 9.024124 & 1 & 31 & 16 & 15 & \# \\
\hline $\mathrm{C}$ & 1.435797 & 1 & 126.9012 & 1 & 51.50711 & 1 & 32 & 21 & 7 & \# \\
\hline $\mathrm{C}$ & 1.511243 & 1 & 123.6824 & 1 & -178.549 & 1 & 3 & 2 & 1 & \# \\
\hline $\mathrm{C}$ & 1.508855 & 1 & 125.3418 & 1 & -1.50007 & 1 & 7 & 6 & 4 & \# \\
\hline $\mathrm{H}$ & 1.066783 & 1 & 128.0559 & 1 & 179.7389 & 1 & 1 & 2 & 3 & \# \\
\hline $\mathrm{H}$ & 1.068757 & 1 & 123.45 & 1 & -179.328 & 1 & 2 & 1 & 5 & \# \\
\hline $\mathrm{H}$ & 1.072154 & 1 & 122.6748 & 1 & -178.419 & 1 & 8 & 7 & 6 & \# \\
\hline $\mathrm{H}$ & 1.066895 & 1 & 128.1974 & 1 & 179.9439 & 1 & 9 & 8 & 7 & \# \\
\hline $\mathrm{H}$ & 1.070805 & 1 & 71.46627 & 1 & -58.2181 & 1 & 11 & 2 & 1 & \# \\
\hline $\mathrm{H}$ & 1.073481 & 1 & 120.1164 & 1 & -100.477 & 1 & 12 & 11 & 2 & \# \\
\hline $\mathrm{H}$ & 1.073376 & 1 & 119.0386 & 1 & -3.26195 & 1 & 17 & 13 & 12 & \# \\
\hline $\mathrm{H}$ & 1.066465 & 1 & 120.7275 & 1 & -178.329 & 1 & 18 & 17 & 13 & \# \\
\hline $\mathrm{H}$ & 1.07359 & 1 & 118.7745 & 1 & -179.878 & 1 & 25 & 24 & 23 & \# \\
\hline $\mathrm{H}$ & 1.068723 & 1 & 119.5799 & 1 & 176.7911 & 1 & 26 & 25 & 24 & \# \\
\hline $\mathrm{H}$ & 1.068282 & 1 & 118.9374 & 1 & -177.992 & 1 & 29 & 28 & 27 & \# \\
\hline $\mathrm{H}$ & 1.073306 & 1 & 120.018 & 1 & 178.9576 & 1 & 30 & 29 & 28 & \# \\
\hline $\mathrm{H}$ & 0.995704 & 1 & 113.2848 & 1 & -152.583 & 1 & 33 & 19 & 18 & $\#$ \\
\hline $\mathrm{H}$ & 0.995528 & 1 & 114.8709 & 1 & 38.33784 & 1 & 34 & 28 & 27 & \# \\
\hline $\mathrm{H}$ & 0.99613 & 1 & 118.075 & 1 & 3.30489 & 1 & 37 & 35 & 33 & \# \\
\hline $\mathrm{H}$ & 0.996224 & 1 & 118.0766 & 1 & 2.339026 & 1 & 39 & 36 & 34 & \# \\
\hline $\mathrm{H}$ & 1.071864 & 1 & 119.5267 & 1 & -3.2825 & 1 & 43 & 41 & 37 & \# \\
\hline $\mathrm{H}$ & 1.070276 & 1 & 119.3483 & 1 & 179.5452 & 1 & 44 & 43 & 41 & \# \\
\hline
\end{tabular}




\begin{tabular}{|c|c|c|c|c|c|c|c|c|c|}
\hline $\mathrm{H}$ & 1.070484 & 1 & 118.6113 & 1 & 179.5985 & 1 & 46 & 45 & 44 \\
\hline $\mathrm{H}$ & 1.072258 & 1 & 120.1823 & 1 & 178.8886 & 1 & 47 & 46 & 45 \\
\hline $\mathrm{H}$ & 1.071983 & 1 & 119.4999 & 1 & -3.27956 & 1 & 48 & 42 & 39 \\
\hline $\mathrm{H}$ & 1.070428 & 1 & 121.5338 & 1 & 179.6838 & 1 & 49 & 48 & 42 \\
\hline $\mathrm{H}$ & 1.070301 & 1 & 121.2009 & 1 & 179.6242 & 1 & 51 & 50 & 49 \\
\hline $\mathrm{H}$ & 1.072183 & 1 & 119.5761 & 1 & 3.243785 & 1 & 52 & 42 & 39 \\
\hline $\mathrm{H}$ & 1.081998 & 1 & 109.2442 & 1 & -54.0118 & 1 & 53 & 31 & 16 \\
\hline $\mathrm{H}$ & 1.077857 & 1 & 103.9059 & 1 & -169.355 & 1 & 53 & 31 & 16 \\
\hline $\mathrm{H}$ & 1.079524 & 1 & 104.7159 & 1 & 134.871 & 1 & 54 & 32 & 21 \\
\hline $\mathrm{H}$ & 1.080657 & 1 & 111.1862 & 1 & 18.54334 & 1 & 54 & 32 & 21 \\
\hline $\mathrm{H}$ & 1.082818 & 1 & 108.56 & 1 & 95.87907 & 1 & 55 & 3 & 2 \\
\hline $\mathrm{H}$ & 1.083465 & 1 & 109.2009 & 1 & -149.021 & 1 & 55 & 3 & 2 \\
\hline $\mathrm{H}$ & 1.080198 & 1 & 110.2509 & 1 & -0.71942 & 1 & 56 & 7 & 6 \\
\hline $\mathrm{H}$ & 1.087982 & 1 & 108.4891 & 1 & 116.163 & 1 & 56 & 7 & 6 \\
\hline $\mathrm{C}$ & 2.445559 & 1 & 145.5189 & 1 & 177.5054 & 1 & 50 & 49 & 48 \\
\hline $\mathrm{H}$ & 1.075971 & 1 & 133.9244 & 1 & 2.495495 & 1 & 89 & 50 & 49 \\
\hline $\mathrm{H}$ & 1.082274 & 1 & 96.56976 & 1 & 127.9977 & 1 & 89 & 50 & 49 \\
\hline $\mathrm{H}$ & 1.082095 & 1 & 95.12625 & 1 & -121.338 & 1 & 89 & 50 & 49 \\
\hline $\mathrm{C}$ & 2.445615 & 1 & 94.20574 & 1 & 178.576 & 1 & 45 & 44 & 43 \\
\hline $\mathrm{H}$ & 1.075982 & 1 & 133.9277 & 1 & 179.6609 & 1 & 93 & 45 & 44 \\
\hline $\mathrm{H}$ & 1.082051 & 1 & 95.0509 & 1 & -56.5815 & 1 & 93 & 45 & 44 \\
\hline $\mathrm{H}$ & 1.082243 & 1 & 96.62872 & 1 & 54.08016 & 1 & 93 & 45 & 44 \\
\hline $\mathrm{O}$ & 1.369861 & 1 & 115.7985 & 1 & -179.824 & 1 & 50 & 49 & 48 \\
\hline $\mathrm{O}$ & 1.369997 & 1 & 123.9556 & 1 & -179.682 & 1 & 45 & 44 & 43 \\
\hline $\mathrm{H}$ & 1.069162 & 1 & 118.2995 & 1 & 70.33293 & 1 & 22 & 21 & 7 \\
\hline $\mathrm{H}$ & 1.067073 & 1 & 118.7364 & 1 & 179.1809 & 1 & 15 & 14 & 13 \\
\hline & 0 & 0 & 0 & 0 & 0 & 0 & 0 & 0 & 0 \\
\hline
\end{tabular}

Total energy $=-3947.181060$ Hartree 
$\mathbf{M}$ analogs; $l=3$

Z-matrices

\begin{tabular}{|c|c|c|c|c|c|c|c|c|c|c|}
\hline $\mathrm{C}$ & 0 & 1 & 0 & 1 & 0 & 1 & 0 & 0 & 0 & \# \\
\hline $\mathrm{C}$ & 1.339687 & 1 & 0 & 1 & 0 & 1 & 1 & 0 & 0 & \# \\
\hline $\mathrm{C}$ & 1.449385 & 1 & 114.9039 & 1 & 0 & 1 & 2 & 1 & 0 & \# \\
\hline $\mathrm{C}$ & 1.353696 & 1 & 112.8741 & 1 & -0.22692 & 1 & 3 & 2 & 1 & \# \\
\hline S & 1.778116 & 1 & 111.5546 & 1 & 0.138277 & 1 & 1 & 2 & 3 & \# \\
\hline $\mathrm{C}$ & 1.462044 & 1 & 131.7026 & 1 & 178.2611 & 1 & 4 & 3 & 2 & \# \\
\hline $\mathrm{C}$ & 1.353676 & 1 & 131.2078 & 1 & 71.03156 & 1 & 6 & 4 & 3 & \# \\
\hline $\mathrm{C}$ & 1.451161 & 1 & 112.7301 & 1 & 175.2172 & 1 & 7 & 6 & 4 & \# \\
\hline $\mathrm{C}$ & 1.339307 & 1 & 114.8367 & 1 & 0.675494 & 1 & 8 & 7 & 6 & $\#$ \\
\hline$S$ & 1.778193 & 1 & 111.6891 & 1 & -0.15803 & 1 & 9 & 8 & 7 & \# \\
\hline $\mathrm{C}$ & 5.386259 & 1 & 117.5066 & 1 & -161.96 & 1 & 3 & 2 & 1 & \# \\
\hline $\mathrm{C}$ & 1.359403 & 1 & 148.8459 & 1 & 63.72032 & 1 & 11 & 3 & 2 & \# \\
\hline $\mathrm{C}$ & 1.415535 & 1 & 120.8437 & 1 & 113.7568 & 1 & 12 & 11 & 3 & \# \\
\hline $\mathrm{C}$ & 1.412186 & 1 & 118.9966 & 1 & -0.25094 & 1 & 13 & 12 & 11 & \# \\
\hline $\mathrm{C}$ & 1.428934 & 1 & 119.7586 & 1 & 1.37285 & 1 & 14 & 13 & 12 & \# \\
\hline $\mathrm{C}$ & 1.369306 & 1 & 118.9755 & 1 & -1.33772 & 1 & 15 & 14 & 13 & \# \\
\hline $\mathrm{C}$ & 1.419504 & 1 & 121.6817 & 1 & 179.8644 & 1 & 13 & 12 & 11 & \# \\
\hline $\mathrm{C}$ & 1.358657 & 1 & 121.4082 & 1 & 179.8706 & 1 & 17 & 13 & 12 & \# \\
\hline $\mathrm{C}$ & 1.414838 & 1 & 119.28 & 1 & -1.12383 & 1 & 18 & 17 & 13 & \# \\
\hline $\mathrm{C}$ & 1.361894 & 1 & 120.7785 & 1 & 1.201307 & 1 & 19 & 18 & 17 & \# \\
\hline $\mathrm{C}$ & 2.497606 & 1 & 114.5584 & 1 & -152.415 & 1 & 15 & 14 & 13 & \# \\
\hline $\mathrm{C}$ & 1.366351 & 1 & 30.6518 & 1 & -110.525 & 1 & 21 & 15 & 14 & \# \\
\hline $\mathrm{C}$ & 1.433562 & 1 & 118.6117 & 1 & -179.81 & 1 & 22 & 21 & 15 & \# \\
\hline $\mathrm{C}$ & 1.412695 & 1 & 120.0456 & 1 & 0.432624 & 1 & 23 & 22 & 21 & \# \\
\hline $\mathrm{C}$ & 1.417973 & 1 & 118.9579 & 1 & -1.58333 & 1 & 24 & 23 & 22 & \# \\
\hline $\mathrm{C}$ & 1.357007 & 1 & 120.4584 & 1 & 1.014008 & 1 & 25 & 24 & 23 & \# \\
\hline $\mathrm{C}$ & 1.420463 & 1 & 121.8717 & 1 & 179.7011 & 1 & 23 & 22 & 21 & \# \\
\hline $\mathrm{C}$ & 1.360144 & 1 & 120.968 & 1 & -178.029 & 1 & 27 & 23 & 22 & \# \\
\hline $\mathrm{C}$ & 1.41182 & 1 & 120.9151 & 1 & -0.67659 & 1 & 28 & 27 & 23 & \# \\
\hline $\mathrm{C}$ & 1.36037 & 1 & 119.4818 & 1 & -0.27502 & 1 & 29 & 28 & 27 & \# \\
\hline $\mathrm{C}$ & 1.511502 & 1 & 120.4368 & 1 & -179.296 & 1 & 3 & 2 & 1 & \# \\
\hline $\mathrm{O}$ & 1.374215 & 1 & 117.6393 & 1 & -175.884 & 1 & 16 & 15 & 14 & \# \\
\hline $\mathrm{O}$ & 1.381035 & 1 & 89.46698 & 1 & 67.90338 & 1 & 21 & 15 & 14 & \# \\
\hline $\mathrm{N}$ & 1.426856 & 1 & 120.6647 & 1 & 176.7116 & 1 & 19 & 18 & 17 & \# \\
\hline $\mathrm{N}$ & 1.43235 & 1 & 119.9426 & 1 & -178.05 & 1 & 28 & 27 & 23 & \# \\
\hline $\mathrm{C}$ & 1.351509 & 1 & 128.3926 & 1 & 57.03629 & 1 & 34 & 19 & 18 & \# \\
\hline
\end{tabular}




\begin{tabular}{|c|c|c|c|c|c|c|c|c|c|c|}
\hline $\mathrm{C}$ & 1.349079 & 1 & 126.3155 & 1 & -90.4329 & 1 & 35 & 28 & 27 & \# \\
\hline $\mathrm{N}$ & 1.351209 & 1 & 113.2141 & 1 & -179.824 & 1 & 36 & 34 & 19 & \# \\
\hline$S$ & 1.733747 & 1 & 124.0255 & 1 & 0.723908 & 1 & 36 & 34 & 19 & \# \\
\hline $\mathrm{N}$ & 1.349495 & 1 & 113.8432 & 1 & -179.758 & 1 & 37 & 35 & 28 & \# \\
\hline$S$ & 1.736584 & 1 & 123.0676 & 1 & 0.077885 & 1 & 37 & 35 & 28 & \# \\
\hline $\mathrm{C}$ & 1.429883 & 1 & 126.4482 & 1 & -178.884 & 1 & 38 & 36 & 34 & \# \\
\hline $\mathrm{C}$ & 1.430349 & 1 & 126.4872 & 1 & -179.503 & 1 & 40 & 37 & 35 & \# \\
\hline $\mathrm{C}$ & 1.381621 & 1 & 120.5184 & 1 & 81.22064 & 1 & 42 & 38 & 36 & $\#$ \\
\hline $\mathrm{C}$ & 1.388734 & 1 & 120.5164 & 1 & 177.6752 & 1 & 44 & 42 & 38 & \# \\
\hline $\mathrm{C}$ & 1.386589 & 1 & 119.4977 & 1 & -0.28306 & 1 & 45 & 44 & 42 & \# \\
\hline $\mathrm{C}$ & 1.39036 & 1 & 120.2179 & 1 & 0.174615 & 1 & 46 & 45 & 44 & \# \\
\hline $\mathrm{C}$ & 1.379967 & 1 & 119.8026 & 1 & 0.216941 & 1 & 47 & 46 & 45 & \# \\
\hline $\mathrm{C}$ & 1.390207 & 1 & 119.7718 & 1 & 100.5971 & 1 & 43 & 40 & 37 & $\#$ \\
\hline $\mathrm{C}$ & 1.379857 & 1 & 120.3275 & 1 & 177.3567 & 1 & 49 & 43 & 40 & \# \\
\hline $\mathrm{C}$ & 1.390513 & 1 & 119.8103 & 1 & 0.467415 & 1 & 50 & 49 & 43 & \# \\
\hline $\mathrm{C}$ & 1.386615 & 1 & 120.2105 & 1 & -0.22047 & 1 & 51 & 50 & 49 & \# \\
\hline $\mathrm{C}$ & 1.381522 & 1 & 120.5534 & 1 & -81.7236 & 1 & 43 & 40 & 37 & \# \\
\hline $\mathrm{C}$ & 1.450729 & 1 & 125.1781 & 1 & -141.623 & 1 & 32 & 16 & 15 & \# \\
\hline $\mathrm{C}$ & 1.45136 & 1 & 120.0275 & 1 & 119.6072 & 1 & 33 & 21 & 15 & \# \\
\hline $\mathrm{C}$ & 1.520521 & 1 & 107.223 & 1 & -139.689 & 1 & 54 & 32 & 16 & \# \\
\hline $\mathrm{C}$ & 1.522641 & 1 & 110.5164 & 1 & -89.4567 & 1 & 55 & 33 & 21 & \# \\
\hline $\mathrm{C}$ & 1.511014 & 1 & 125.2891 & 1 & -4.22686 & 1 & 7 & 6 & 4 & \# \\
\hline $\mathrm{H}$ & 1.066781 & 1 & 128.2266 & 1 & -179.757 & 1 & 1 & 2 & 3 & \# \\
\hline $\mathrm{H}$ & 1.071412 & 1 & 123.0752 & 1 & 179.9396 & 1 & 2 & 1 & 5 & \# \\
\hline $\mathrm{H}$ & 1.070187 & 1 & 122.6503 & 1 & -177.707 & 1 & 8 & 7 & 6 & \# \\
\hline $\mathrm{H}$ & 1.066774 & 1 & 128.1327 & 1 & -179.574 & 1 & 9 & 8 & 7 & \# \\
\hline $\mathrm{H}$ & 1.07077 & 1 & 51.36276 & 1 & -21.9916 & 1 & 11 & 3 & 2 & \# \\
\hline $\mathrm{H}$ & 1.073567 & 1 & 120.1348 & 1 & -65.3502 & 1 & 12 & 11 & 3 & \# \\
\hline $\mathrm{H}$ & 1.07347 & 1 & 118.6909 & 1 & -0.37992 & 1 & 17 & 13 & 12 & \# \\
\hline $\mathrm{H}$ & 1.06886 & 1 & 121.1934 & 1 & -179.727 & 1 & 18 & 17 & 13 & \# \\
\hline $\mathrm{H}$ & 1.070846 & 1 & 119.6762 & 1 & -178.722 & 1 & 20 & 19 & 18 & \# \\
\hline $\mathrm{H}$ & 1.073448 & 1 & 119.1094 & 1 & -178.649 & 1 & 25 & 24 & 23 & \# \\
\hline $\mathrm{H}$ & 1.071895 & 1 & 120.7763 & 1 & -178.532 & 1 & 26 & 25 & 24 & \# \\
\hline $\mathrm{H}$ & 1.0697 & 1 & 119.7031 & 1 & 0.845693 & 1 & 27 & 23 & 22 & \# \\
\hline $\mathrm{H}$ & 1.071782 & 1 & 119.2467 & 1 & 178.9269 & 1 & 29 & 28 & 27 & \# \\
\hline $\mathrm{H}$ & 1.073441 & 1 & 120.2237 & 1 & 179.8831 & 1 & 30 & 29 & 28 & \# \\
\hline $\mathrm{H}$ & 1.079714 & 1 & 109.5836 & 1 & 121.461 & 1 & 31 & 3 & 2 & \# \\
\hline $\mathrm{H}$ & 1.086371 & 1 & 107.8343 & 1 & 6.208713 & 1 & 31 & 3 & 2 & \# \\
\hline
\end{tabular}




\begin{tabular}{|c|c|c|c|c|c|c|c|c|c|c|}
\hline $\mathrm{H}$ & 0.995439 & 1 & 114.1505 & 1 & -128.507 & 1 & 34 & 19 & 18 & \# \\
\hline $\mathrm{H}$ & 0.996081 & 1 & 115.56 & 1 & 89.4206 & 1 & 35 & 28 & 27 & \# \\
\hline $\mathrm{H}$ & 0.995892 & 1 & 117.9883 & 1 & 2.109817 & 1 & 38 & 36 & 34 & \# \\
\hline $\mathrm{H}$ & 0.996045 & 1 & 117.928 & 1 & -0.27839 & 1 & 40 & 37 & 35 & \# \\
\hline $\mathrm{H}$ & 1.071673 & 1 & 119.5129 & 1 & -3.37472 & 1 & 44 & 42 & 38 & \# \\
\hline $\mathrm{H}$ & 1.070297 & 1 & 119.33 & 1 & 179.4418 & 1 & 45 & 44 & 42 & \# \\
\hline $\mathrm{H}$ & 1.070525 & 1 & 118.6281 & 1 & 179.5566 & 1 & 47 & 46 & 45 & \# \\
\hline $\mathrm{H}$ & 1.072383 & 1 & 120.1613 & 1 & 178.77 & 1 & 48 & 47 & 46 & \# \\
\hline $\mathrm{H}$ & 1.072395 & 1 & 119.5053 & 1 & -3.29137 & 1 & 49 & 43 & 40 & \# \\
\hline $\mathrm{H}$ & 1.070516 & 1 & 121.569 & 1 & 179.8697 & 1 & 50 & 49 & 43 & \# \\
\hline $\mathrm{H}$ & 1.07028 & 1 & 121.1658 & 1 & 179.5806 & 1 & 52 & 51 & 50 & \# \\
\hline $\mathrm{H}$ & 1.07166 & 1 & 119.5376 & 1 & 3.310212 & 1 & 53 & 43 & 40 & \# \\
\hline $\mathrm{H}$ & 1.079512 & 1 & 107.4408 & 1 & 99.9969 & 1 & 54 & 32 & 16 & \# \\
\hline $\mathrm{H}$ & 1.081995 & 1 & 110.2405 & 1 & -18.0908 & 1 & 54 & 32 & 16 & \# \\
\hline $\mathrm{H}$ & 1.079068 & 1 & 105.0193 & 1 & 149.9861 & 1 & 55 & 33 & 21 & \# \\
\hline $\mathrm{H}$ & 1.08021 & 1 & 108.9573 & 1 & 33.59835 & 1 & 55 & 33 & 21 & \# \\
\hline $\mathrm{H}$ & 1.081664 & 1 & 108.6591 & 1 & -60.4328 & 1 & 56 & 54 & 32 & \# \\
\hline $\mathrm{H}$ & 1.085864 & 1 & 108.7932 & 1 & -176.849 & 1 & 56 & 54 & 32 & \# \\
\hline $\mathrm{H}$ & 1.079333 & 1 & 108.5348 & 1 & 50.21801 & 1 & 57 & 55 & 33 & \# \\
\hline $\mathrm{H}$ & 1.084252 & 1 & 107.7449 & 1 & -65.3406 & 1 & 57 & 55 & 33 & \# \\
\hline $\mathrm{H}$ & 1.087839 & 1 & 109.1514 & 1 & 130.4664 & 1 & 58 & 7 & 6 & \# \\
\hline $\mathrm{H}$ & 1.082042 & 1 & 108.6958 & 1 & 15.08358 & 1 & 58 & 7 & 6 & \# \\
\hline $\mathrm{C}$ & 2.44569 & 1 & 145.5559 & 1 & 177.1592 & 1 & 51 & 50 & 49 & \# \\
\hline $\mathrm{H}$ & 1.075982 & 1 & 133.9322 & 1 & 2.589815 & 1 & 97 & 51 & 50 & \# \\
\hline $\mathrm{H}$ & 1.082232 & 1 & 96.60079 & 1 & 128.1384 & 1 & 97 & 51 & 50 & \# \\
\hline $\mathrm{H}$ & 1.082042 & 1 & 95.07495 & 1 & -121.202 & 1 & 97 & 51 & 50 & \# \\
\hline $\mathrm{C}$ & 2.445458 & 1 & 94.18891 & 1 & 178.4746 & 1 & 46 & 45 & 44 & \# \\
\hline $\mathrm{H}$ & 1.076037 & 1 & 133.963 & 1 & 179.6405 & 1 & 101 & 46 & 45 & \# \\
\hline $\mathrm{H}$ & 1.082058 & 1 & 94.96459 & 1 & -56.6816 & 1 & 101 & 46 & 45 & \# \\
\hline $\mathrm{H}$ & 1.08227 & 1 & 96.69818 & 1 & 53.95717 & 1 & 101 & 46 & 45 & \# \\
\hline $\mathrm{O}$ & 1.370084 & 1 & 115.8293 & 1 & 179.957 & 1 & 51 & 50 & 49 & \# \\
\hline $\mathrm{O}$ & 1.370557 & 1 & 123.9436 & 1 & -179.619 & 1 & 46 & 45 & 44 & \# \\
\hline & 0 & 0 & 0 & 0 & 0 & 0 & 0 & 0 & 0 & \\
\hline
\end{tabular}

Total energy $=-4025.215433$ Hartree 
M analogs; $l=4$

Z-matrices

\begin{tabular}{|c|c|c|c|c|c|c|c|c|c|c|}
\hline $\mathrm{C}$ & 0 & 1 & 0 & 1 & 0 & 1 & 0 & 0 & 0 & \# \\
\hline $\mathrm{C}$ & 1.340526 & 1 & 0 & 1 & 0 & 1 & 1 & 0 & 0 & \# \\
\hline $\mathrm{C}$ & 1.450322 & 1 & 114.5641 & 1 & 0 & 1 & 2 & 1 & 0 & \# \\
\hline $\mathrm{C}$ & 1.352773 & 1 & 112.6913 & 1 & 0.712849 & 1 & 3 & 2 & 1 & \# \\
\hline$S$ & 1.779672 & 1 & 111.862 & 1 & -0.37911 & 1 & 1 & 2 & 3 & \# \\
\hline $\mathrm{C}$ & 1.460887 & 1 & 129.2602 & 1 & 174.3365 & 1 & 4 & 3 & 2 & \# \\
\hline $\mathrm{C}$ & 1.3494 & 1 & 130.621 & 1 & 93.47397 & 1 & 6 & 4 & 3 & \# \\
\hline $\mathrm{C}$ & 1.451115 & 1 & 113.1395 & 1 & 179.3376 & 1 & 7 & 6 & 4 & \# \\
\hline $\mathrm{C}$ & 1.338886 & 1 & 114.5516 & 1 & -0.16723 & 1 & 8 & 7 & 6 & \# \\
\hline$S$ & 1.783526 & 1 & 111.7588 & 1 & -0.00743 & 1 & 9 & 8 & 7 & \# \\
\hline $\mathrm{C}$ & 6.705485 & 1 & 88.10865 & 1 & -104.847 & 1 & 3 & 2 & 1 & \# \\
\hline $\mathrm{C}$ & 1.356398 & 1 & 139.8417 & 1 & 127.5521 & 1 & 11 & 3 & 2 & \# \\
\hline $\mathrm{C}$ & 1.421627 & 1 & 121.0582 & 1 & 56.34052 & 1 & 12 & 11 & 3 & \# \\
\hline $\mathrm{C}$ & 1.411562 & 1 & 118.8761 & 1 & 1.801913 & 1 & 13 & 12 & 11 & \# \\
\hline $\mathrm{C}$ & 1.422839 & 1 & 119.1761 & 1 & -1.52943 & 1 & 14 & 13 & 12 & \# \\
\hline $\mathrm{C}$ & 1.363166 & 1 & 120.0993 & 1 & -0.36661 & 1 & 15 & 14 & 13 & \# \\
\hline $\mathrm{C}$ & 1.415432 & 1 & 121.6484 & 1 & -177.628 & 1 & 13 & 12 & 11 & \# \\
\hline $\mathrm{C}$ & 1.358499 & 1 & 121.0269 & 1 & 177.9389 & 1 & 17 & 13 & 12 & \# \\
\hline $\mathrm{C}$ & 1.413494 & 1 & 119.7446 & 1 & 0.594526 & 1 & 18 & 17 & 13 & \# \\
\hline $\mathrm{C}$ & 1.375049 & 1 & 121.3691 & 1 & 2.100528 & 1 & 19 & 18 & 17 & \# \\
\hline $\mathrm{C}$ & 4.30885 & 1 & 101.199 & 1 & -126.257 & 1 & 20 & 19 & 18 & \# \\
\hline $\mathrm{C}$ & 1.362806 & 1 & 0.37864 & 1 & -27.3773 & 1 & 21 & 20 & 19 & \# \\
\hline $\mathrm{C}$ & 1.422254 & 1 & 120.1596 & 1 & -91.5108 & 1 & 22 & 21 & 20 & \# \\
\hline $\mathrm{C}$ & 1.411025 & 1 & 119.256 & 1 & -1.02411 & 1 & 23 & 22 & 21 & \# \\
\hline $\mathrm{C}$ & 1.421006 & 1 & 118.8011 & 1 & -1.24554 & 1 & 24 & 23 & 22 & \# \\
\hline $\mathrm{C}$ & 1.356752 & 1 & 121.0412 & 1 & 1.941141 & 1 & 25 & 24 & 23 & \# \\
\hline $\mathrm{C}$ & 1.431149 & 1 & 121.538 & 1 & 176.1604 & 1 & 23 & 22 & 21 & \# \\
\hline $\mathrm{C}$ & 1.374988 & 1 & 119.1918 & 1 & -174.814 & 1 & 27 & 23 & 22 & \# \\
\hline $\mathrm{C}$ & 1.414032 & 1 & 121.321 & 1 & -4.01148 & 1 & 28 & 27 & 23 & \# \\
\hline $\mathrm{C}$ & 1.358306 & 1 & 119.7829 & 1 & 2.619206 & 1 & 29 & 28 & 27 & \# \\
\hline $\mathrm{O}$ & 1.364731 & 1 & 124.5742 & 1 & -177.266 & 1 & 16 & 15 & 14 & \# \\
\hline $\mathrm{O}$ & 1.366745 & 1 & 124.9786 & 1 & 68.54997 & 1 & 21 & 20 & 19 & \# \\
\hline $\mathrm{N}$ & 1.425369 & 1 & 119.4689 & 1 & 179.0564 & 1 & 19 & 18 & 17 & \# \\
\hline $\mathrm{N}$ & 1.424494 & 1 & 119.0495 & 1 & 178.8762 & 1 & 28 & 27 & 23 & \# \\
\hline $\mathrm{C}$ & 1.354261 & 1 & 127.4338 & 1 & 58.82877 & 1 & 33 & 19 & 18 & \# \\
\hline $\mathrm{C}$ & 1.355311 & 1 & 127.4816 & 1 & -124.975 & 1 & 34 & 28 & 27 & \# \\
\hline
\end{tabular}




\begin{tabular}{|c|c|c|c|c|c|c|c|c|c|c|}
\hline $\mathrm{N}$ & 1.346886 & 1 & 113.2944 & 1 & 177.2203 & 1 & 35 & 33 & 19 & \# \\
\hline S & 1.737575 & 1 & 124.0481 & 1 & -2.12055 & 1 & 35 & 33 & 19 & \# \\
\hline $\mathrm{N}$ & 1.346967 & 1 & 113.2229 & 1 & 176.5001 & 1 & 36 & 34 & 28 & \# \\
\hline S & 1.736547 & 1 & 124.1671 & 1 & -2.82251 & 1 & 36 & 34 & 28 & \# \\
\hline $\mathrm{C}$ & 1.430971 & 1 & 126.3683 & 1 & -177.168 & 1 & 37 & 35 & 33 & \# \\
\hline $\mathrm{C}$ & 1.431081 & 1 & 126.2799 & 1 & -176.675 & 1 & 39 & 36 & 34 & \# \\
\hline $\mathrm{C}$ & 1.381468 & 1 & 120.2204 & 1 & 84.37337 & 1 & 41 & 37 & 35 & \# \\
\hline $\mathrm{C}$ & 1.388891 & 1 & 120.4558 & 1 & 177.6603 & 1 & 43 & 41 & 37 & $\#$ \\
\hline $\mathrm{C}$ & 1.386586 & 1 & 119.4731 & 1 & -0.14765 & 1 & 44 & 43 & 41 & $\#$ \\
\hline $\mathrm{C}$ & 1.390818 & 1 & 120.2458 & 1 & 0.105513 & 1 & 45 & 44 & 43 & \# \\
\hline $\mathrm{C}$ & 1.379619 & 1 & 119.8236 & 1 & 0.144074 & 1 & 46 & 45 & 44 & \# \\
\hline $\mathrm{C}$ & 1.390989 & 1 & 119.9836 & 1 & 86.86543 & 1 & 42 & 39 & 36 & \# \\
\hline $\mathrm{C}$ & 1.378821 & 1 & 120.1684 & 1 & 177.5075 & 1 & 48 & 42 & 39 & \# \\
\hline $\mathrm{C}$ & 1.391487 & 1 & 119.8837 & 1 & 0.076788 & 1 & 49 & 48 & 42 & \# \\
\hline $\mathrm{C}$ & 1.385939 & 1 & 120.2616 & 1 & 0.021052 & 1 & 50 & 49 & 48 & \# \\
\hline $\mathrm{C}$ & 1.38051 & 1 & 120.2036 & 1 & -95.4183 & 1 & 42 & 39 & 36 & \# \\
\hline $\mathrm{H}$ & 1.066827 & 1 & 127.9798 & 1 & 179.4426 & 1 & 1 & 2 & 3 & \# \\
\hline $\mathrm{H}$ & 1.069666 & 1 & 122.387 & 1 & 179.5277 & 1 & 2 & 1 & 5 & \# \\
\hline $\mathrm{H}$ & 1.071235 & 1 & 121.9825 & 1 & 179.667 & 1 & 8 & 7 & 6 & \# \\
\hline $\mathrm{H}$ & 1.066923 & 1 & 128.2222 & 1 & 179.8917 & 1 & 9 & 8 & 7 & \# \\
\hline $\mathrm{H}$ & 1.07076 & 1 & 83.48345 & 1 & -6.53906 & 1 & 11 & 3 & 2 & \# \\
\hline $\mathrm{H}$ & 1.073501 & 1 & 120.1884 & 1 & -122.578 & 1 & 12 & 11 & 3 & \# \\
\hline $\mathrm{H}$ & 1.073309 & 1 & 118.9781 & 1 & -1.29521 & 1 & 17 & 13 & 12 & \# \\
\hline $\mathrm{H}$ & 1.068611 & 1 & 121.2148 & 1 & -178.282 & 1 & 18 & 17 & 13 & \# \\
\hline $\mathrm{H}$ & 1.073485 & 1 & 118.7816 & 1 & -179.142 & 1 & 25 & 24 & 23 & \# \\
\hline $\mathrm{H}$ & 1.07084 & 1 & 122.0187 & 1 & 179.0547 & 1 & 26 & 25 & 24 & \# \\
\hline $\mathrm{H}$ & 1.068396 & 1 & 119.0223 & 1 & -178.698 & 1 & 29 & 28 & 27 & \# \\
\hline $\mathrm{H}$ & 1.073307 & 1 & 119.9924 & 1 & 179.5115 & 1 & 30 & 29 & 28 & \# \\
\hline $\mathrm{H}$ & 0.995682 & 1 & 114.7922 & 1 & -134.012 & 1 & 33 & 19 & 18 & \# \\
\hline $\mathrm{H}$ & 0.995679 & 1 & 114.6805 & 1 & 41.27516 & 1 & 34 & 28 & 27 & \# \\
\hline $\mathrm{H}$ & 0.996178 & 1 & 118.0299 & 1 & 2.012159 & 1 & 37 & 35 & 33 & \# \\
\hline $\mathrm{H}$ & 0.996154 & 1 & 118.0752 & 1 & 1.469607 & 1 & 39 & 36 & 34 & $\#$ \\
\hline $\mathrm{H}$ & 1.072077 & 1 & 119.5454 & 1 & -3.20852 & 1 & 43 & 41 & 37 & \# \\
\hline $\mathrm{H}$ & 1.070253 & 1 & 119.3368 & 1 & 179.5775 & 1 & 44 & 43 & 41 & \# \\
\hline $\mathrm{H}$ & 1.070475 & 1 & 118.606 & 1 & 179.5981 & 1 & 46 & 45 & 44 & \# \\
\hline $\mathrm{H}$ & 1.072211 & 1 & 120.2164 & 1 & 178.9332 & 1 & 47 & 46 & 45 & \# \\
\hline $\mathrm{H}$ & 1.072059 & 1 & 119.4938 & 1 & -3.35661 & 1 & 48 & 42 & 39 & \# \\
\hline $\mathrm{H}$ & 1.070426 & 1 & 121.5209 & 1 & 179.6507 & 1 & 49 & 48 & 42 & \# \\
\hline
\end{tabular}




\begin{tabular}{|c|c|c|c|c|c|c|c|c|c|c|}
\hline $\mathrm{H}$ & 1.070308 & 1 & 121.2095 & 1 & 179.6105 & 1 & 51 & 50 & 49 & \# \\
\hline $\mathrm{H}$ & 1.072234 & 1 & 119.5834 & 1 & 3.275401 & 1 & 52 & 42 & 39 & \# \\
\hline $\mathrm{C}$ & 2.445546 & 1 & 145.5137 & 1 & 177.5652 & 1 & 50 & 49 & 48 & \# \\
\hline $\mathrm{H}$ & 1.075979 & 1 & 133.9337 & 1 & 2.478085 & 1 & 77 & 50 & 49 & \# \\
\hline $\mathrm{H}$ & 1.082284 & 1 & 96.55438 & 1 & 127.9663 & 1 & 77 & 50 & 49 & \# \\
\hline $\mathrm{H}$ & 1.082104 & 1 & 95.13947 & 1 & -121.376 & 1 & 77 & 50 & 49 & \# \\
\hline $\mathrm{C}$ & 2.445667 & 1 & 94.19109 & 1 & 178.6928 & 1 & 45 & 44 & 43 & \# \\
\hline $\mathrm{H}$ & 1.07597 & 1 & 133.9214 & 1 & 179.6819 & 1 & 81 & 45 & 44 & \# \\
\hline $\mathrm{H}$ & 1.082034 & 1 & 95.11272 & 1 & -56.4884 & 1 & 81 & 45 & 44 & \# \\
\hline $\mathrm{H}$ & 1.082211 & 1 & 96.56616 & 1 & 54.17341 & 1 & 81 & 45 & 44 & \# \\
\hline $\mathrm{O}$ & 1.369973 & 1 & 115.7905 & 1 & -179.8 & 1 & 50 & 49 & 48 & \# \\
\hline $\mathrm{O}$ & 1.369859 & 1 & 123.948 & 1 & -179.7 & 1 & 45 & 44 & 43 & \# \\
\hline $\mathrm{H}$ & 1.067995 & 1 & 120.8776 & 1 & 86.97923 & 1 & 22 & 21 & 20 & \# \\
\hline $\mathrm{H}$ & 1.068835 & 1 & 119.2036 & 1 & 179.9312 & 1 & 15 & 14 & 13 & \# \\
\hline $\mathrm{C}$ & 1.505966 & 1 & 126.1189 & 1 & -2.06112 & 1 & 7 & 6 & 4 & \# \\
\hline $\mathrm{H}$ & 1.084177 & 1 & 107.8638 & 1 & -123.783 & 1 & 89 & 7 & 6 & \# \\
\hline $\mathrm{H}$ & 1.083377 & 1 & 109.9876 & 1 & -7.6425 & 1 & 89 & 7 & 6 & \# \\
\hline $\mathrm{C}$ & 1.515876 & 1 & 123.937 & 1 & 178.3718 & 1 & 3 & 2 & 1 & \# \\
\hline $\mathrm{H}$ & 1.083182 & 1 & 108.6957 & 1 & 148.2038 & 1 & 92 & 3 & 2 & \# \\
\hline $\mathrm{H}$ & 1.087267 & 1 & 108.3846 & 1 & -97.5995 & 1 & 92 & 3 & 2 & \# \\
\hline $\mathrm{C}$ & 1.442227 & 1 & 122.6832 & 1 & 13.17783 & 1 & 31 & 16 & 15 & $\#$ \\
\hline $\mathrm{H}$ & 1.082565 & 1 & 109.5316 & 1 & -72.1651 & 1 & 95 & 31 & 16 & \# \\
\hline $\mathrm{H}$ & 1.083134 & 1 & 108.8916 & 1 & 46.87634 & 1 & 95 & 31 & 16 & \# \\
\hline $\mathrm{C}$ & 1.541076 & 1 & 116.1459 & 1 & 23.54784 & 1 & 92 & 3 & 2 & \# \\
\hline $\mathrm{H}$ & 1.086917 & 1 & 107.5482 & 1 & -178.451 & 1 & 98 & 92 & 3 & \# \\
\hline $\mathrm{H}$ & 1.085529 & 1 & 109.0209 & 1 & -64.4739 & 1 & 98 & 92 & 3 & \# \\
\hline $\mathrm{C}$ & 1.518203 & 1 & 105.4138 & 1 & 167.1303 & 1 & 95 & 31 & 16 & \# \\
\hline $\mathrm{H}$ & 1.084412 & 1 & 107.7261 & 1 & 60.91116 & 1 & 101 & 95 & 31 & \# \\
\hline $\mathrm{H}$ & 1.082046 & 1 & 107.6623 & 1 & -53.7626 & 1 & 101 & 95 & 31 & \# \\
\hline $\mathrm{C}$ & 1.446447 & 1 & 124.0167 & 1 & -4.26873 & 1 & 32 & 21 & 20 & \# \\
\hline $\mathrm{H}$ & 1.078745 & 1 & 103.0407 & 1 & -159.636 & 1 & 104 & 32 & 21 & \# \\
\hline $\mathrm{H}$ & 1.082078 & 1 & 109.5975 & 1 & -44.334 & 1 & 104 & 32 & 21 & $\#$ \\
\hline $\mathrm{C}$ & 1.543898 & 1 & 113.7727 & 1 & 115.0521 & 1 & 89 & 7 & 6 & \# \\
\hline $\mathrm{H}$ & 1.084527 & 1 & 108.4122 & 1 & -77.6533 & 1 & 107 & 89 & 7 & \# \\
\hline $\mathrm{H}$ & 1.083611 & 1 & 109.79 & 1 & 38.36905 & 1 & 107 & 89 & 7 & \# \\
\hline $\mathrm{C}$ & 1.529765 & 1 & 113.6695 & 1 & 81.44507 & 1 & 104 & 32 & 21 & \# \\
\hline $\mathrm{H}$ & 1.086194 & 1 & 106.1672 & 1 & 167.968 & 1 & 110 & 104 & 32 & \# \\
\hline $\mathrm{H}$ & 1.084702 & 1 & 110.7246 & 1 & -77.5204 & 1 & 110 & 104 & 32 & \# \\
\hline
\end{tabular}


0

$0 \quad 0$

0

0

0

0

0

$\begin{array}{ll}0 & 0\end{array}$

Total energy $=-4103.263843$ Hartree 
$\mathbf{M}$ analogs; $l=5$

Z-matrices

\begin{tabular}{|c|c|c|c|c|c|c|c|c|c|}
\hline $\mathrm{C}$ & 0 & 1 & 0 & 1 & 0 & 1 & 0 & 0 & $0 \#$ \\
\hline $\mathrm{C}$ & 1.339867 & 1 & 0 & 1 & 0 & 1 & 1 & 0 & 0\# \\
\hline $\mathrm{C}$ & 1.450879 & & 114.5571 & 1 & 0 & 1 & 2 & 1 & 0\# \\
\hline $\mathrm{C}$ & 1.351525 & & 112.838 & 1 & 0.202177 & 1 & 3 & 2 & $1 \#$ \\
\hline S & 1.781466 & & 111.8388 & 1 & -0.24373 & 1 & 1 & 2 & $3 \#$ \\
\hline $\mathrm{C}$ & 1.46093 & & 129.7104 & 1 & 177.5068 & 1 & 4 & 3 & $2 \#$ \\
\hline $\mathrm{C}$ & 1.350347 & & 129.8826 & 1 & 97.60499 & 1 & 6 & 4 & $3 \#$ \\
\hline $\mathrm{C}$ & 1.450311 & 1 & 113.0264 & 1 & 177.8651 & 1 & 7 & 6 & 4\# \\
\hline $\mathrm{C}$ & 1.339653 & 1 & 114.5639 & 1 & 0.070118 & 1 & 8 & 7 & $6 \#$ \\
\hline S & 1.782071 & 1 & 111.7347 & 1 & -0.13121 & 1 & 9 & 8 & 7\# \\
\hline $\mathrm{C}$ & 9.961672 & & 93.04082 & 1 & -42.6449 & 1 & 7 & 6 & 4\# \\
\hline $\mathrm{C}$ & 1.356497 & 1 & 121.7514 & 1 & -169.796 & 1 & 11 & 7 & 6\# \\
\hline $\mathrm{C}$ & 1.421667 & 1 & 121.1119 & 1 & 48.25728 & 1 & 12 & 11 & 7\# \\
\hline $\mathrm{C}$ & 1.411 & 1 & 118.9586 & 1 & 1.135857 & 1 & 13 & 12 & $11 \#$ \\
\hline $\mathrm{C}$ & 1.422872 & & 119.051 & 1 & -0.79844 & 1 & 14 & 13 & $12 \#$ \\
\hline $\mathrm{C}$ & 1.363343 & 1 & 120.1827 & 1 & -0.54051 & 1 & 15 & 14 & $13 \#$ \\
\hline $\mathrm{C}$ & 1.414389 & 1 & 121.7476 & 1 & -178.964 & 1 & 13 & 12 & $11 \#$ \\
\hline $\mathrm{C}$ & 1.358801 & 1 & 121.2693 & 1 & 178.8783 & 1 & 17 & 13 & $12 \#$ \\
\hline $\mathrm{C}$ & 1.414536 & & 119.774 & 1 & -0.12947 & 1 & 18 & 17 & $13 \#$ \\
\hline $\mathrm{C}$ & 1.377917 & 1 & 121.0247 & 1 & 2.342071 & 1 & 19 & 18 & $17 \#$ \\
\hline $\mathrm{C}$ & 4.326219 & 1 & 101.5812 & 1 & -126.737 & 1 & 20 & 19 & $18 \#$ \\
\hline $\mathrm{C}$ & 1.362031 & 1 & 0.296877 & 1 & 137.8522 & 1 & 21 & 20 & $19 \#$ \\
\hline $\mathrm{C}$ & 1.419878 & 1 & 120.3792 & 1 & 72.36456 & 1 & 22 & 21 & $20 \#$ \\
\hline $\mathrm{C}$ & 1.413353 & 1 & 118.7978 & 1 & -0.25898 & 1 & 23 & 22 & $21 \#$ \\
\hline $\mathrm{C}$ & 1.417736 & 1 & 119.1318 & 1 & -2.01491 & 1 & 24 & 23 & $22 \#$ \\
\hline $\mathrm{C}$ & 1.360569 & 1 & 120.988 & 1 & 2.132448 & 1 & 25 & 24 & $23 \#$ \\
\hline $\mathrm{C}$ & 1.434372 & 1 & 121.9694 & 1 & 176.9255 & 1 & 23 & 22 & $21 \#$ \\
\hline $\mathrm{C}$ & 1.374046 & 1 & 119.0877 & 1 & -174.524 & 1 & 27 & 23 & $22 \#$ \\
\hline $\mathrm{C}$ & 1.416307 & 1 & 121.3837 & 1 & -4.04945 & 1 & 28 & 27 & $23 \#$ \\
\hline $\mathrm{C}$ & 1.356481 & 1 & 119.8846 & 1 & 2.419666 & 1 & 29 & 28 & $27 \#$ \\
\hline $\mathrm{O}$ & 1.366182 & 1 & 124.4382 & 1 & -177.697 & 1 & 16 & 15 & $14 \#$ \\
\hline $\mathrm{O}$ & 1.381003 & 1 & 119.4765 & 1 & 66.6313 & 1 & 21 & 20 & $19 \#$ \\
\hline$N$ & 1.421592 & 1 & 120.3562 & 1 & 178.1015 & 1 & 19 & 18 & $17 \#$ \\
\hline $\mathrm{N}$ & 1.423975 & 1 & 119.0758 & 1 & 179.1148 & 1 & 28 & 27 & $23 \#$ \\
\hline $\mathrm{C}$ & 1.357124 & 1 & 129.2697 & 1 & 48.55988 & 1 & 33 & 19 & \\
\hline
\end{tabular}




\begin{tabular}{|c|c|c|c|c|c|c|c|c|}
\hline $\mathrm{C}$ & 1.354826 & $1 \quad 127.4852$ & 1 & -125.332 & 1 & 34 & 28 & $27 \#$ \\
\hline $\mathrm{N}$ & 1.349414 & 1112.6703 & 1 & 177.2532 & 1 & 35 & 33 & $19 \#$ \\
\hline S & 1.73261 & 1124.9934 & 1 & -1.81204 & 1 & 35 & 33 & $19 \#$ \\
\hline $\mathrm{N}$ & 1.346456 & $1 \quad 113.2682$ & 1 & 176.2508 & 1 & 36 & 34 & $28 \#$ \\
\hline S & 1.73765 & 1124.1316 & 1 & -3.10692 & 1 & 36 & 34 & $28 \#$ \\
\hline $\mathrm{C}$ & 1.430592 & $1 \quad 126.2748$ & 1 & -176.973 & 1 & 37 & 35 & $33 \#$ \\
\hline $\mathrm{C}$ & 1.431136 & $\begin{array}{ll}1 & 126.2887\end{array}$ & 1 & -176.656 & 1 & 39 & 36 & $34 \#$ \\
\hline $\mathrm{C}$ & 1.380499 & 1120.1089 & 1 & -97.7316 & 1 & 41 & 37 & $35 \#$ \\
\hline $\mathrm{C}$ & 1.390049 & $1 \quad 120.5504$ & 1 & -177.32 & 1 & 43 & 41 & $37 \#$ \\
\hline $\mathrm{C}$ & 1.385699 & $1 \quad 119.3802$ & 1 & -0.24455 & 1 & 44 & 43 & $41 \#$ \\
\hline $\mathrm{C}$ & 1.391521 & $1 \quad 120.2583$ & 1 & 0.042271 & 1 & 45 & 44 & $43 \#$ \\
\hline $\mathrm{C}$ & 1.378687 & $1 \quad 119.8992$ & 1 & 0.069307 & 1 & 46 & 45 & $44 \#$ \\
\hline $\mathrm{C}$ & 1.390973 & $1 \quad 119.9615$ & 1 & 87.273 & 1 & 42 & 39 & $36 \#$ \\
\hline $\mathrm{C}$ & 1.378855 & $1 \quad 120.164$ & 1 & 177.5126 & 1 & 48 & 42 & $39 \#$ \\
\hline $\mathrm{C}$ & 1.391471 & $\begin{array}{ll}1 & 119.8819\end{array}$ & 1 & 0.084775 & 1 & 49 & 48 & $42 \#$ \\
\hline $\mathrm{C}$ & 1.385971 & 1120.2637 & 1 & 0.008528 & 1 & 50 & 49 & $48 \#$ \\
\hline $\mathrm{C}$ & 1.380506 & $1 \quad 120.2168$ & 1 & -95.016 & 1 & 42 & 39 & $36 \#$ \\
\hline $\mathrm{H}$ & 1.066897 & 1128.0719 & 1 & -179.993 & 1 & 1 & 2 & $3 \#$ \\
\hline $\mathrm{H}$ & 1.06996 & 1122.62 & 1 & 179.325 & 1 & 2 & 1 & $5 \#$ \\
\hline $\mathrm{H}$ & 1.071545 & $\begin{array}{ll}1 & 122.4728\end{array}$ & 1 & -179.487 & 1 & 8 & 7 & $6 \#$ \\
\hline $\mathrm{H}$ & 1.066889 & $1 \quad 128.1735$ & 1 & -179.856 & 1 & 9 & 8 & 7\# \\
\hline $\mathrm{H}$ & 1.070773 & $\begin{array}{ll}1 & 100.9869\end{array}$ & 1 & 50.84886 & 1 & 11 & 7 & $6 \#$ \\
\hline $\mathrm{H}$ & 1.073548 & 1120.1756 & 1 & -130.981 & 1 & 12 & 11 & 7\# \\
\hline $\mathrm{H}$ & 1.073384 & $1 \quad 118.97$ & 1 & -0.95453 & 1 & 17 & 13 & $12 \#$ \\
\hline $\mathrm{H}$ & 1.067021 & 1120.9803 & 1 & -179.115 & 1 & 18 & 17 & $13 \#$ \\
\hline $\mathrm{H}$ & 1.073532 & 1118.7638 & 1 & -178.252 & 1 & 25 & 24 & $23 \#$ \\
\hline $\mathrm{H}$ & 1.071855 & $1 \quad 120.955$ & 1 & -179.398 & 1 & 26 & 25 & $24 \#$ \\
\hline $\mathrm{H}$ & 1.068383 & 1118.9395 & 1 & -178.578 & 1 & 29 & 28 & $27 \#$ \\
\hline $\mathrm{H}$ & 1.073215 & $1 \quad 120.041$ & 1 & 179.8439 & 1 & 30 & 29 & $28 \#$ \\
\hline $\mathrm{H}$ & 0.995547 & 1113.7295 & 1 & -144.232 & 1 & 33 & 19 & $18 \#$ \\
\hline $\mathrm{H}$ & 0.995864 & $1 \quad 114.5758$ & 1 & 40.6875 & 1 & 34 & 28 & $27 \#$ \\
\hline $\mathrm{H}$ & 0.996018 & $1 \quad 118.1371$ & 1 & 1.437209 & 1 & 37 & 35 & $33 \#$ \\
\hline $\mathrm{H}$ & 0.996231 & 1118.0585 & 1 & 1.293162 & 1 & 39 & 36 & $34 \#$ \\
\hline $\mathrm{H}$ & 1.072338 & $\begin{array}{ll}1 & 119.5688\end{array}$ & 1 & 3.389458 & 1 & 43 & 41 & $37 \#$ \\
\hline $\mathrm{H}$ & 1.070342 & $\begin{array}{ll}1 & 119.3961\end{array}$ & 1 & -179.803 & 1 & 44 & 43 & $41 \#$ \\
\hline $\mathrm{H}$ & 1.070429 & $1 \quad 118.5873$ & 1 & -179.487 & 1 & 46 & 45 & $44 \#$ \\
\hline $\mathrm{H}$ & 1.071866 & 1120.3433 & 1 & -178.993 & 1 & 47 & 46 & $45 \#$ \\
\hline
\end{tabular}




\begin{tabular}{|c|c|c|c|c|c|c|c|c|c|}
\hline $\mathrm{H}$ & 1.07209 & 1 & 119.4966 & 1 & -3.31503 & 1 & 48 & 42 & $39 \#$ \\
\hline $\mathrm{H}$ & 1.070427 & 1 & 121.52 & 1 & 179.6752 & 1 & 49 & 48 & $42 \#$ \\
\hline $\mathrm{H}$ & 1.070306 & 1 & 121.2092 & 1 & 179.626 & 1 & 51 & 50 & $49 \#$ \\
\hline $\mathrm{H}$ & 1.072214 & 1 & 119.5842 & 1 & 3.249497 & 1 & 52 & 42 & $39 \#$ \\
\hline $\mathrm{C}$ & 2.445553 & 1 & 145.5189 & 1 & 177.6839 & 1 & 50 & 49 & $48 \#$ \\
\hline $\mathrm{H}$ & 1.07598 & 1 & 133.9348 & 1 & 2.346621 & 1 & 77 & 50 & $49 \#$ \\
\hline $\mathrm{H}$ & 1.082281 & 1 & 96.51947 & 1 & 127.7935 & 1 & 77 & 50 & $49 \#$ \\
\hline $\mathrm{H}$ & 1.082109 & 1 & 95.17417 & 1 & -121.549 & 1 & 77 & 50 & $49 \#$ \\
\hline $\mathrm{C}$ & 2.445321 & 1 & 94.19933 & 1 & -178.359 & 1 & 45 & 44 & $43 \#$ \\
\hline $\mathrm{H}$ & 1.076021 & 1 & 133.9628 & 1 & -179.599 & 1 & 81 & 45 & $44 \#$ \\
\hline $\mathrm{H}$ & 1.082331 & 1 & 96.64804 & 1 & -53.9831 & 1 & 81 & 45 & 44\# \\
\hline $\mathrm{H}$ & 1.082128 & 1 & 95.03523 & 1 & 56.65793 & 1 & 81 & 45 & $44 \#$ \\
\hline $\mathrm{O}$ & 1.369977 & 1 & 115.7901 & 1 & -179.817 & 1 & 50 & 49 & $48 \#$ \\
\hline $\mathrm{O}$ & 1.370377 & 1 & 123.9457 & 1 & 179.8285 & 1 & 45 & 44 & $43 \#$ \\
\hline $\mathrm{H}$ & 1.069901 & 1 & 118.9551 & 1 & -108.598 & 1 & 22 & 21 & $20 \#$ \\
\hline $\mathrm{H}$ & 1.068478 & 1 & 119.0929 & 1 & 179.4668 & 1 & 15 & 14 & $13 \#$ \\
\hline $\mathrm{C}$ & 2.535548 & 1 & 130.7651 & 1 & -45.7815 & 1 & 7 & 6 & 4\# \\
\hline $\mathrm{H}$ & 1.086229 & 1 & 91.59985 & 1 & -27.8022 & 1 & 89 & 7 & $6 \#$ \\
\hline $\mathrm{C}$ & 2.562382 & 1 & 104.6417 & 1 & -147.916 & 1 & 3 & 2 & $1 \#$ \\
\hline $\mathrm{H}$ & 1.085249 & 1 & 131.5397 & 1 & 178.8248 & 1 & 91 & 3 & $2 \#$ \\
\hline $\mathrm{C}$ & 1.440387 & 1 & 122.0127 & 1 & 9.565219 & 1 & 31 & 16 & $15 \#$ \\
\hline $\mathrm{H}$ & 1.082729 & 1 & 109.4938 & 1 & -71.9471 & 1 & 93 & 31 & $16 \#$ \\
\hline $\mathrm{H}$ & 1.082754 & 1 & 108.7141 & 1 & 46.79581 & 1 & 93 & 31 & $16 \#$ \\
\hline $\mathrm{C}$ & 1.539057 & 1 & 114.0998 & 1 & -30.4927 & 1 & 91 & 3 & $2 \#$ \\
\hline $\mathrm{H}$ & 1.087449 & 1 & 109.1152 & 1 & -108.64 & 1 & 96 & 91 & $3 \#$ \\
\hline $\mathrm{H}$ & 1.08462 & 1 & 109.6543 & 1 & 7.03 & 1 & 96 & 91 & $3 \#$ \\
\hline $\mathrm{C}$ & 1.51811 & 1 & 106.2723 & 1 & 166.9783 & 1 & 93 & 31 & $16 \#$ \\
\hline $\mathrm{H}$ & 1.084098 & 1 & 108.2489 & 1 & 60.40937 & 1 & 99 & 93 & $31 \#$ \\
\hline $\mathrm{H}$ & 1.085013 & 1 & 108.1016 & 1 & -54.9473 & 1 & 99 & 93 & $31 \#$ \\
\hline $\mathrm{C}$ & 1.449343 & 1 & 119.1134 & 1 & 116.734 & 1 & 32 & 21 & $20 \#$ \\
\hline $\mathrm{H}$ & 1.082143 & 1 & 109.0832 & 1 & 48.45414 & 1 & 102 & 32 & $21 \#$ \\
\hline $\mathrm{H}$ & 1.078372 & 1 & 104.4483 & 1 & 164.3247 & 1 & 102 & 32 & $21 \#$ \\
\hline $\mathrm{C}$ & 1.535376 & 1 & 145.977 & 1 & 101.5571 & 1 & 89 & 7 & $6 \#$ \\
\hline $\mathrm{H}$ & 1.086096 & 1 & 108.7932 & 1 & -58.6648 & 1 & 105 & 89 & 7\# \\
\hline $\mathrm{H}$ & 1.088439 & 1 & 109.6199 & 1 & 56.95441 & 1 & 105 & 89 & 7\# \\
\hline $\mathrm{C}$ & 1.524006 & 1 & 111.6645 & 1 & -75.3992 & 1 & 102 & 32 & $21 \#$ \\
\hline $\mathrm{H}$ & 1.084242 & 1 & 108.1496 & 1 & -62.9549 & 1 & 108 & 102 & $32 \#$ \\
\hline
\end{tabular}




\begin{tabular}{|c|c|c|c|c|c|c|c|c|}
\hline $\mathrm{H}$ & 1.085472 & $\begin{array}{ll}1 & 109.3985\end{array}$ & & 52.78884 & 1 & 108 & 102 & $32 \#$ \\
\hline $\mathrm{H}$ & 1.085361 & 187.99809 & 1 & -134.116 & 1 & 89 & 7 & $6 \#$ \\
\hline $\mathrm{C}$ & 1.508056 & $1 \quad 125.48$ & 1 & -2.50877 & 1 & 7 & 6 & 4\# \\
\hline $\mathrm{H}$ & 1.082659 & 1109.8046 & 1 & 10.05712 & 1 & 112 & 7 & $6 \#$ \\
\hline $\mathrm{H}$ & 1.087465 & $\begin{array}{ll}1 & 108.8889\end{array}$ & 1 & 126.5993 & 1 & 112 & 7 & $6 \#$ \\
\hline $\mathrm{H}$ & 1.085992 & 179.34164 & 1 & 76.32795 & 1 & 91 & 3 & $2 \#$ \\
\hline $\mathrm{C}$ & 1.510153 & $\begin{array}{ll}1 & 122.8127\end{array}$ & 1 & -179.152 & 1 & 3 & 2 & $1 \#$ \\
\hline $\mathrm{H}$ & 1.081222 & 1108.4477 & 1 & -173.219 & 1 & 116 & 3 & $2 \#$ \\
\hline $\mathrm{H}$ & 1.086798 & $\begin{array}{ll}1 & 109.3295\end{array}$ & 1 & -57.6929 & 1 & 116 & 3 & $2 \#$ \\
\hline & 0 & 0 & 0 & 0 & 0 & 0 & 0 & 0 \\
\hline
\end{tabular}

Total energy $=-4181.295910$ Hartree 
M analogs; $l=3$ with phthalate

Z-matrices

\begin{tabular}{|c|c|c|c|c|c|c|c|c|c|}
\hline $\mathrm{C}$ & 0 & 1 & 0 & 1 & 0 & 1 & 0 & 0 & 0 \\
\hline $\mathrm{C}$ & 1.339347 & 1 & 0 & 1 & 0 & 1 & 1 & 0 & 0 \\
\hline $\mathrm{C}$ & 1.449717 & 1 & 115.0824 & 1 & 0 & 1 & 2 & 1 & 0 \\
\hline $\mathrm{C}$ & 1.355062 & 1 & 112.838 & 1 & -0.62623 & 1 & 3 & 2 & 1 \\
\hline S & 1.780267 & 1 & 111.4053 & 1 & -0.26636 & 1 & 1 & 2 & 3 \\
\hline $\mathrm{C}$ & 1.459548 & 1 & 130.0218 & 1 & 178.6838 & 1 & 4 & 3 & 2 \\
\hline $\mathrm{C}$ & 1.353224 & 1 & 130.4344 & 1 & 125.5033 & 1 & 6 & 4 & 3 \\
\hline $\mathrm{C}$ & 1.449462 & 1 & 112.7816 & 1 & -179.744 & 1 & 7 & 6 & 4 \\
\hline $\mathrm{C}$ & 1.338799 & 1 & 115.0553 & 1 & -0.62317 & 1 & 8 & 7 & 6 \\
\hline$S$ & 1.780563 & 1 & 111.4644 & 1 & -0.1337 & 1 & 9 & 8 & 7 \\
\hline $\mathrm{C}$ & 5.731898 & 1 & 92.01569 & 1 & -108.579 & 1 & 3 & 2 & 1 \\
\hline $\mathrm{C}$ & 1.358022 & 1 & 138.2978 & 1 & 77.59434 & 1 & 11 & 3 & 2 \\
\hline $\mathrm{C}$ & 1.416079 & 1 & 120.6122 & 1 & 86.84458 & 1 & 12 & 11 & 3 \\
\hline $\mathrm{C}$ & 1.414546 & 1 & 119.1208 & 1 & 1.250509 & 1 & 13 & 12 & 11 \\
\hline $\mathrm{C}$ & 1.435062 & 1 & 119.7794 & 1 & -0.32826 & 1 & 14 & 13 & 12 \\
\hline $\mathrm{C}$ & 1.367235 & 1 & 118.51 & 1 & -1.41273 & 1 & 15 & 14 & 13 \\
\hline $\mathrm{C}$ & 1.418039 & 1 & 121.6127 & 1 & -178.631 & 1 & 13 & 12 & 11 \\
\hline $\mathrm{C}$ & 1.359456 & 1 & 120.9275 & 1 & 178.7913 & 1 & 17 & 13 & 12 \\
\hline $\mathrm{C}$ & 1.413626 & 1 & 120.0231 & 1 & 0.17766 & 1 & 18 & 17 & 13 \\
\hline $\mathrm{C}$ & 1.36085 & 1 & 120.301 & 1 & 1.114968 & 1 & 19 & 18 & 17 \\
\hline $\mathrm{C}$ & 2.4864 & 1 & 124.828 & 1 & -147.395 & 1 & 15 & 14 & 13 \\
\hline $\mathrm{C}$ & 1.366897 & 1 & 31.28652 & 1 & -92.2434 & 1 & 21 & 15 & 14 \\
\hline $\mathrm{C}$ & 1.435729 & 1 & 118.5161 & 1 & -179.181 & 1 & 22 & 21 & 15 \\
\hline $\mathrm{C}$ & 1.414194 & 1 & 119.3984 & 1 & 1.043024 & 1 & 23 & 22 & 21 \\
\hline $\mathrm{C}$ & 1.416518 & 1 & 119.4288 & 1 & 0.074741 & 1 & 24 & 23 & 22 \\
\hline $\mathrm{C}$ & 1.358079 & 1 & 120.5804 & 1 & -0.88605 & 1 & 25 & 24 & 23 \\
\hline $\mathrm{C}$ & 1.422844 & 1 & 122.4038 & 1 & -177.544 & 1 & 23 & 22 & 21 \\
\hline $\mathrm{C}$ & 1.361117 & 1 & 121.2257 & 1 & 177.8561 & 1 & 27 & 23 & 22 \\
\hline $\mathrm{C}$ & 1.414527 & 1 & 120.2329 & 1 & 1.540963 & 1 & 28 & 27 & 23 \\
\hline $\mathrm{C}$ & 1.357943 & 1 & 120.0604 & 1 & -0.90027 & 1 & 29 & 28 & 27 \\
\hline $\mathrm{C}$ & 1.51734 & 1 & 120.4939 & 1 & 178.4056 & 1 & 3 & 2 & 1 \\
\hline $\mathrm{O}$ & 1.382681 & 1 & 119.7464 & 1 & -178.614 & 1 & 16 & 15 & 14 \\
\hline $\mathrm{O}$ & 1.390345 & 1 & 89.99511 & 1 & 88.09207 & 1 & 21 & 15 & 14 \\
\hline $\mathrm{N}$ & 1.418779 & 1 & 118.6106 & 1 & 176.7941 & 1 & 19 & 18 & 17 \\
\hline $\mathrm{N}$ & 1.423509 & 1 & 120.3962 & 1 & -172.78 & 1 & 28 & 27 & 23 \\
\hline $\mathrm{C}$ & 1.337762 & 1 & 126.3484 & 1 & 107.3367 & 1 & 34 & 19 & 18 \\
\hline
\end{tabular}




\begin{tabular}{|c|c|c|c|c|c|c|c|c|c|c|}
\hline $\mathrm{C}$ & 1.339259 & 1 & 126.0646 & 1 & -100.721 & 1 & 35 & 28 & 27 & \# \\
\hline $\mathrm{N}$ & 1.342533 & 1 & 113.4363 & 1 & 175.0898 & 1 & 36 & 34 & 19 & \# \\
\hline$S$ & 1.775078 & 1 & 121.8012 & 1 & -4.70943 & 1 & 36 & 34 & 19 & \# \\
\hline $\mathrm{N}$ & 1.34416 & 1 & 113.5381 & 1 & 179.6899 & 1 & 37 & 35 & 28 & \# \\
\hline S & 1.770392 & 1 & 121.0176 & 1 & 0.316768 & 1 & 37 & 35 & 28 & \# \\
\hline $\mathrm{C}$ & 1.411175 & 1 & 131.0247 & 1 & 179.3656 & 1 & 38 & 36 & 34 & $\#$ \\
\hline $\mathrm{C}$ & 1.410916 & 1 & 131.5916 & 1 & 179.7743 & 1 & 40 & 37 & 35 & \# \\
\hline $\mathrm{C}$ & 1.39115 & 1 & 116.1935 & 1 & 148.241 & 1 & 42 & 38 & 36 & \# \\
\hline $\mathrm{C}$ & 1.388792 & 1 & 121.075 & 1 & 176.8402 & 1 & 44 & 42 & 38 & \# \\
\hline $\mathrm{C}$ & 1.383902 & 1 & 119.6866 & 1 & 0.097455 & 1 & 45 & 44 & 42 & \# \\
\hline $\mathrm{C}$ & 1.385586 & 1 & 119.7588 & 1 & -0.75562 & 1 & 46 & 45 & 44 & $\#$ \\
\hline $\mathrm{C}$ & 1.383813 & 1 & 120.5427 & 1 & 0.244269 & 1 & 47 & 46 & 45 & \# \\
\hline $\mathrm{C}$ & 1.402035 & 1 & 115.7176 & 1 & 155.623 & 1 & 43 & 40 & 37 & \# \\
\hline $\mathrm{C}$ & 1.379205 & 1 & 120.9227 & 1 & 177.8103 & 1 & 49 & 43 & 40 & \# \\
\hline $\mathrm{C}$ & 1.388056 & 1 & 120.1077 & 1 & 0.019922 & 1 & 50 & 49 & 43 & \# \\
\hline $\mathrm{C}$ & 1.380683 & 1 & 119.6625 & 1 & -0.76991 & 1 & 51 & 50 & 49 & \# \\
\hline $\mathrm{C}$ & 1.386619 & 1 & 125.7854 & 1 & -28 & 1 & 43 & 40 & 37 & \# \\
\hline $\mathrm{C}$ & 1.439964 & 1 & 119.6551 & 1 & 124.5095 & 1 & 32 & 16 & 15 & \# \\
\hline $\mathrm{C}$ & 1.448519 & 1 & 118.9485 & 1 & -82.9331 & 1 & 33 & 21 & 15 & \# \\
\hline $\mathrm{C}$ & 1.517673 & 1 & 107.8938 & 1 & -137.846 & 1 & 54 & 32 & 16 & \# \\
\hline $\mathrm{C}$ & 1.518919 & 1 & 107.3538 & 1 & 174.0409 & 1 & 55 & 33 & 21 & $\#$ \\
\hline $\mathrm{C}$ & 1.514814 & 1 & 127.0672 & 1 & 2.62498 & 1 & 7 & 6 & 4 & \# \\
\hline $\mathrm{H}$ & 1.066802 & 1 & 128.382 & 1 & 178.926 & 1 & 1 & 2 & 3 & $\#$ \\
\hline $\mathrm{H}$ & 1.071676 & 1 & 123.0483 & 1 & -179.806 & 1 & 2 & 1 & 5 & \# \\
\hline $\mathrm{H}$ & 1.071324 & 1 & 121.8664 & 1 & 178.8017 & 1 & 8 & 7 & 6 & $\#$ \\
\hline $\mathrm{H}$ & 1.06672 & 1 & 128.3651 & 1 & 179.0782 & 1 & 9 & 8 & 7 & \# \\
\hline $\mathrm{H}$ & 1.072607 & 1 & 69.83913 & 1 & -36.819 & 1 & 11 & 3 & 2 & \# \\
\hline $\mathrm{H}$ & 1.074287 & 1 & 120.414 & 1 & -93.3248 & 1 & 12 & 11 & 3 & $\#$ \\
\hline $\mathrm{H}$ & 1.074394 & 1 & 118.7058 & 1 & -1.23492 & 1 & 17 & 13 & 12 & $\#$ \\
\hline $\mathrm{H}$ & 1.071326 & 1 & 121.4706 & 1 & 179.7408 & 1 & 18 & 17 & 13 & \# \\
\hline $\mathrm{H}$ & 1.068851 & 1 & 118.8014 & 1 & 178.693 & 1 & 20 & 19 & 18 & $\#$ \\
\hline $\mathrm{H}$ & 1.074293 & 1 & 118.8946 & 1 & 179.6273 & 1 & 25 & 24 & 23 & \# \\
\hline $\mathrm{H}$ & 1.071971 & 1 & 121.8868 & 1 & 179.8104 & 1 & 26 & 25 & 24 & \# \\
\hline $\mathrm{H}$ & 1.068973 & 1 & 120.0345 & 1 & -0.74892 & 1 & 27 & 23 & 22 & $\#$ \\
\hline $\mathrm{H}$ & 1.071027 & 1 & 118.5239 & 1 & 179.6278 & 1 & 29 & 28 & 27 & \# \\
\hline $\mathrm{H}$ & 1.074401 & 1 & 120.3657 & 1 & -179.424 & 1 & 30 & 29 & 28 & $\#$ \\
\hline $\mathrm{H}$ & 1.082706 & 1 & 109.381 & 1 & -123.861 & 1 & 31 & 3 & 2 & \# \\
\hline $\mathrm{H}$ & 1.086654 & 1 & 107.7374 & 1 & -8.9958 & 1 & 31 & 3 & 2 & \# \\
\hline
\end{tabular}




\begin{tabular}{|c|c|c|c|c|c|c|c|c|c|c|}
\hline $\mathrm{H}$ & 1.027514 & 1 & 113.7762 & 1 & -74.2615 & 1 & 34 & 19 & 18 & \# \\
\hline $\mathrm{H}$ & 1.023686 & 1 & 113.9745 & 1 & 83.07781 & 1 & 35 & 28 & 27 & \# \\
\hline $\mathrm{H}$ & 1.023031 & 1 & 116.6123 & 1 & -8.50928 & 1 & 38 & 36 & 34 & \# \\
\hline $\mathrm{H}$ & 1.022099 & 1 & 116.5299 & 1 & -5.2289 & 1 & 40 & 37 & 35 & \# \\
\hline $\mathrm{H}$ & 1.072281 & 1 & 118.6656 & 1 & -1.59037 & 1 & 44 & 42 & 38 & \# \\
\hline $\mathrm{H}$ & 1.071495 & 1 & 118.8201 & 1 & -179.84 & 1 & 45 & 44 & 42 & \# \\
\hline $\mathrm{H}$ & 1.07191 & 1 & 118.7336 & 1 & -179.373 & 1 & 47 & 46 & 45 & \# \\
\hline $\mathrm{H}$ & 1.066837 & 1 & 119.9015 & 1 & 179.9502 & 1 & 48 & 47 & 46 & \# \\
\hline $\mathrm{H}$ & 1.0721 & 1 & 118.5953 & 1 & -0.67985 & 1 & 49 & 43 & 40 & \# \\
\hline $\mathrm{H}$ & 1.071716 & 1 & 120.9349 & 1 & -179.97 & 1 & 50 & 49 & 43 & \# \\
\hline $\mathrm{H}$ & 1.071891 & 1 & 121.2913 & 1 & -179.449 & 1 & 52 & 51 & 50 & \# \\
\hline $\mathrm{H}$ & 1.065944 & 1 & 119.9319 & 1 & 3.22027 & 1 & 53 & 43 & 40 & \# \\
\hline $\mathrm{H}$ & 1.081506 & 1 & 109.7594 & 1 & -16.6998 & 1 & 54 & 32 & 16 & \# \\
\hline $\mathrm{H}$ & 1.085309 & 1 & 108.3841 & 1 & 101.7405 & 1 & 54 & 32 & 16 & \# \\
\hline $\mathrm{H}$ & 1.07851 & 1 & 109.3223 & 1 & 53.79313 & 1 & 55 & 33 & 21 & \# \\
\hline $\mathrm{H}$ & 1.083997 & 1 & 109.2602 & 1 & -64.0402 & 1 & 55 & 33 & 21 & \# \\
\hline $\mathrm{H}$ & 1.084206 & 1 & 108.1157 & 1 & -51.4842 & 1 & 56 & 54 & 32 & \# \\
\hline $\mathrm{H}$ & 1.07903 & 1 & 108.4155 & 1 & 64.72593 & 1 & 56 & 54 & 32 & \# \\
\hline $\mathrm{H}$ & 1.082127 & 1 & 108.0359 & 1 & -40.3004 & 1 & 57 & 55 & 33 & \# \\
\hline $\mathrm{H}$ & 1.086152 & 1 & 108.1098 & 1 & -155.786 & 1 & 57 & 55 & 33 & \# \\
\hline $\mathrm{H}$ & 1.085853 & 1 & 107.5136 & 1 & 161.0798 & 1 & 58 & 7 & 6 & \# \\
\hline $\mathrm{H}$ & 1.082849 & 1 & 109.288 & 1 & 46.42425 & 1 & 58 & 7 & 6 & \# \\
\hline $\mathrm{C}$ & 2.439155 & 1 & 146.42 & 1 & 178.9269 & 1 & 51 & 50 & 49 & \# \\
\hline $\mathrm{H}$ & 1.078345 & 1 & 135.3794 & 1 & 0.096761 & 1 & 97 & 51 & 50 & \# \\
\hline $\mathrm{H}$ & 1.083776 & 1 & 95.8701 & 1 & 125.534 & 1 & 97 & 51 & 50 & \# \\
\hline $\mathrm{H}$ & 1.083644 & 1 & 95.22989 & 1 & -124.597 & 1 & 97 & 51 & 50 & \# \\
\hline $\mathrm{C}$ & 2.439436 & 1 & 93.78519 & 1 & 179.0778 & 1 & 46 & 45 & 44 & \# \\
\hline $\mathrm{H}$ & 1.078285 & 1 & 135.3365 & 1 & 179.9297 & 1 & 101 & 46 & 45 & \# \\
\hline $\mathrm{H}$ & 1.083452 & 1 & 95.22903 & 1 & -55.4078 & 1 & 101 & 46 & 45 & \# \\
\hline $\mathrm{H}$ & 1.083489 & 1 & 95.85471 & 1 & 54.49362 & 1 & 101 & 46 & 45 & \# \\
\hline $\mathrm{O}$ & 1.390103 & 1 & 116.3195 & 1 & 179.5499 & 1 & 51 & 50 & 49 & \# \\
\hline $\mathrm{O}$ & 1.38956 & 1 & 123.9067 & 1 & 179.7316 & 1 & 46 & 45 & 44 & \# \\
\hline $\mathrm{C}$ & 5.507898 & 1 & 121.8833 & 1 & 1.848199 & 1 & 38 & 36 & 34 & \# \\
\hline $\mathrm{C}$ & 1.393316 & 1 & 61.25904 & 1 & -27.8694 & 1 & 107 & 38 & 36 & \# \\
\hline $\mathrm{C}$ & 1.396626 & 1 & 119.111 & 1 & -177.238 & 1 & 108 & 107 & 38 & \# \\
\hline $\mathrm{C}$ & 1.393401 & 1 & 119.0125 & 1 & -2.71521 & 1 & 109 & 108 & 107 & \# \\
\hline $\mathrm{C}$ & 1.38498 & 1 & 121.4377 & 1 & 1.69459 & 1 & 110 & 109 & 108 & \# \\
\hline $\mathrm{C}$ & 1.384914 & 1 & 177.2832 & 1 & 165.9776 & 1 & 107 & 38 & 36 & \# \\
\hline
\end{tabular}




$\begin{array}{rrrrrrrrrrr}\mathrm{H} & 1.071722 & 1 & 56.75773 & 1 & 151.1625 & 1 & 107 & 38 & 36 & \# \\ \mathrm{H} & 1.075105 & 1 & 120.012 & 1 & 179.713 & 1 & 111 & 110 & 109 & \# \\ \mathrm{H} & 1.075241 & 1 & 119.997 & 1 & -14.6371 & 1 & 112 & 107 & 38 & \# \\ \mathrm{C} & 1.50757 & 1 & 116.0523 & 1 & 7.118239 & 1 & 108 & 107 & 38 & \# \\ \mathrm{C} & 1.509839 & 1 & 124.5201 & 1 & 171.9831 & 1 & 109 & 108 & 107 & \# \\ \mathrm{O} & 1.255877 & 1 & 118.6066 & 1 & -35.8178 & 1 & 117 & 109 & 108 & \# \\ \mathrm{O} & 1.272482 & 1 & 116.8056 & 1 & 150.3147 & 1 & 117 & 109 & 108 & \# \\ \mathrm{O} & 1.257754 & 1 & 118.218 & 1 & 130.0018 & 1 & 116 & 108 & 107 & \# \\ \mathrm{O} & 1.270865 & 1 & 117.113 & 1 & -43.5314 & 1 & 116 & 108 & 107 & \# \\ \mathrm{H} & 1.071153 & 1 & 117.7857 & 1 & -178.534 & 1 & 110 & 109 & 108 & \# \\ & 0 & 0 & 0 & 0 & 0 & 0 & 0 & 0 & 0 & 0\end{array}$

Total energy $=-4629.813776$ Hartree 
$\mathbf{M}$ analogs; $l=3$ with isophthalate

Z-matrices

\begin{tabular}{|c|c|c|c|c|c|c|c|c|c|}
\hline $\mathrm{C}$ & 0 & 1 & 0 & 1 & 0 & 1 & 0 & 0 & 0 \\
\hline $\mathrm{C}$ & 1.339355 & 1 & 0 & 1 & 0 & 1 & 1 & 0 & 0 \\
\hline $\mathrm{C}$ & 1.449651 & 1 & 115.0702 & 1 & 0 & 1 & 2 & 1 & 0 \\
\hline $\mathrm{C}$ & 1.355226 & 1 & 112.8453 & 1 & -0.60598 & 1 & 3 & 2 & 1 \\
\hline S & 1.780108 & 1 & 111.4108 & 1 & -0.28213 & 1 & 1 & 2 & 3 \\
\hline $\mathrm{C}$ & 1.459742 & 1 & 130.0565 & 1 & 178.5942 & 1 & 4 & 3 & 2 \\
\hline $\mathrm{C}$ & 1.353413 & 1 & 130.4467 & 1 & 126.1913 & 1 & 6 & 4 & 3 \\
\hline $\mathrm{C}$ & 1.449349 & 1 & 112.7882 & 1 & -179.983 & 1 & 7 & 6 & 4 \\
\hline $\mathrm{C}$ & 1.338886 & 1 & 115.0585 & 1 & -0.57415 & 1 & 8 & 7 & 6 \\
\hline$S$ & 1.780104 & 1 & 111.4513 & 1 & -0.16874 & 1 & 9 & 8 & 7 \\
\hline $\mathrm{C}$ & 5.731726 & 1 & 91.5173 & 1 & -108.324 & 1 & 3 & 2 & 1 \\
\hline $\mathrm{C}$ & 1.358543 & 1 & 136.8172 & 1 & 79.35818 & 1 & 11 & 3 & 2 \\
\hline $\mathrm{C}$ & 1.415948 & 1 & 120.5937 & 1 & 85.08556 & 1 & 12 & 11 & 3 \\
\hline $\mathrm{C}$ & 1.413424 & 1 & 119.0812 & 1 & 1.961524 & 1 & 13 & 12 & 11 \\
\hline $\mathrm{C}$ & 1.433706 & 1 & 119.8251 & 1 & -1.3004 & 1 & 14 & 13 & 12 \\
\hline $\mathrm{C}$ & 1.367073 & 1 & 118.5723 & 1 & -0.91899 & 1 & 15 & 14 & 13 \\
\hline $\mathrm{C}$ & 1.419572 & 1 & 121.6126 & 1 & -177.04 & 1 & 13 & 12 & 11 \\
\hline $\mathrm{C}$ & 1.358387 & 1 & 121.0105 & 1 & 177.5723 & 1 & 17 & 13 & 12 \\
\hline $\mathrm{C}$ & 1.415046 & 1 & 119.9416 & 1 & 0.73416 & 1 & 18 & 17 & 13 \\
\hline $\mathrm{C}$ & 1.36057 & 1 & 120.3129 & 1 & 1.010945 & 1 & 19 & 18 & 17 \\
\hline $\mathrm{C}$ & 2.487816 & 1 & 122.4525 & 1 & -146.798 & 1 & 15 & 14 & 13 \\
\hline $\mathrm{C}$ & 1.36693 & 1 & 31.19371 & 1 & -95.7039 & 1 & 21 & 15 & 14 \\
\hline $\mathrm{C}$ & 1.434251 & 1 & 118.6057 & 1 & -179.621 & 1 & 22 & 21 & 15 \\
\hline $\mathrm{C}$ & 1.413427 & 1 & 119.4879 & 1 & -0.18718 & 1 & 23 & 22 & 21 \\
\hline $\mathrm{C}$ & 1.416689 & 1 & 119.344 & 1 & 0.146472 & 1 & 24 & 23 & 22 \\
\hline $\mathrm{C}$ & 1.358544 & 1 & 120.5964 & 1 & 0.039776 & 1 & 25 & 24 & 23 \\
\hline $\mathrm{C}$ & 1.422762 & 1 & 122.285 & 1 & 179.6182 & 1 & 23 & 22 & 21 \\
\hline $\mathrm{C}$ & 1.361791 & 1 & 121.2581 & 1 & 179.9094 & 1 & 27 & 23 & 22 \\
\hline $\mathrm{C}$ & 1.415473 & 1 & 120.1978 & 1 & 0.25878 & 1 & 28 & 27 & 23 \\
\hline $\mathrm{C}$ & 1.357933 & 1 & 119.9965 & 1 & -0.00703 & 1 & 29 & 28 & 27 \\
\hline $\mathrm{C}$ & 1.517171 & 1 & 120.4997 & 1 & 178.495 & 1 & 3 & 2 & 1 \\
\hline $\mathrm{O}$ & 1.381268 & 1 & 119.6935 & 1 & -178.282 & 1 & 16 & 15 & 14 \\
\hline $\mathrm{O}$ & 1.389507 & 1 & 90.00699 & 1 & 86.86031 & 1 & 21 & 15 & 14 \\
\hline $\mathrm{N}$ & 1.420223 & 1 & 119.6053 & 1 & 177.9455 & 1 & 19 & 18 & 17 \\
\hline $\mathrm{N}$ & 1.421514 & 1 & 119.89 & 1 & -175.478 & 1 & 28 & 27 & 23 \\
\hline $\mathrm{C}$ & 1.346425 & 1 & 125.8694 & 1 & 83.43894 & 1 & 34 & 19 & 18 \\
\hline
\end{tabular}




\begin{tabular}{|c|c|c|c|c|c|c|c|c|c|c|}
\hline $\mathrm{C}$ & 1.345992 & 1 & 126.4029 & 1 & -108.775 & 1 & 35 & 28 & 27 & $\#$ \\
\hline $\mathrm{N}$ & 1.33834 & 1 & 112.5593 & 1 & 179.8236 & 1 & 36 & 34 & 19 & $\#$ \\
\hline S & 1.768319 & 1 & 121.191 & 1 & -0.75495 & 1 & 36 & 34 & 19 & \# \\
\hline $\mathrm{N}$ & 1.340884 & 1 & 112.7079 & 1 & -179.891 & 1 & 37 & 35 & 28 & \# \\
\hline S & 1.765029 & 1 & 121.499 & 1 & -0.74713 & 1 & 37 & 35 & 28 & \# \\
\hline $\mathrm{C}$ & 1.413086 & 1 & 132.4915 & 1 & 177.2895 & 1 & 38 & 36 & 34 & $\#$ \\
\hline $\mathrm{C}$ & 1.414007 & 1 & 131.1754 & 1 & 179.9062 & 1 & 40 & 37 & 35 & \# \\
\hline $\mathrm{C}$ & 1.392629 & 1 & 115.679 & 1 & -154.76 & 1 & 42 & 38 & 36 & \# \\
\hline $\mathrm{C}$ & 1.388064 & 1 & 120.7351 & 1 & -177.484 & 1 & 44 & 42 & 38 & $\#$ \\
\hline $\mathrm{C}$ & 1.384642 & 1 & 119.8749 & 1 & -0.11713 & 1 & 45 & 44 & 42 & $\#$ \\
\hline $\mathrm{C}$ & 1.384734 & 1 & 119.7459 & 1 & 0.629011 & 1 & 46 & 45 & 44 & \# \\
\hline $\mathrm{C}$ & 1.384805 & 1 & 120.56 & 1 & -0.15911 & 1 & 47 & 46 & 45 & \# \\
\hline $\mathrm{C}$ & 1.399704 & 1 & 116.3835 & 1 & -149.626 & 1 & 43 & 40 & 37 & $\#$ \\
\hline $\mathrm{C}$ & 1.38067 & 1 & 120.3856 & 1 & -177.412 & 1 & 49 & 43 & 40 & $\#$ \\
\hline $\mathrm{C}$ & 1.38792 & 1 & 120.2252 & 1 & -0.10043 & 1 & 50 & 49 & 43 & $\#$ \\
\hline $\mathrm{C}$ & 1.381787 & 1 & 119.8513 & 1 & 0.860234 & 1 & 51 & 50 & 49 & \# \\
\hline $\mathrm{C}$ & 1.38538 & 1 & 124.4994 & 1 & 34.3996 & 1 & 43 & 40 & 37 & \# \\
\hline $\mathrm{C}$ & 1.442067 & 1 & 119.7518 & 1 & 122.9076 & 1 & 32 & 16 & 15 & $\#$ \\
\hline $\mathrm{C}$ & 1.447986 & 1 & 119.7344 & 1 & -83.8497 & 1 & 33 & 21 & 15 & \# \\
\hline $\mathrm{C}$ & 1.517891 & 1 & 108.043 & 1 & -134.507 & 1 & 54 & 32 & 16 & \# \\
\hline $\mathrm{C}$ & 1.519088 & 1 & 107.3404 & 1 & 173.5764 & 1 & 55 & 33 & 21 & \# \\
\hline $\mathrm{C}$ & 1.51488 & 1 & 127.0997 & 1 & 2.357162 & 1 & 7 & 6 & 4 & \# \\
\hline $\mathrm{H}$ & 1.06678 & 1 & 128.3896 & 1 & 178.988 & 1 & 1 & 2 & 3 & \# \\
\hline $\mathrm{H}$ & 1.071673 & 1 & 123.0463 & 1 & -179.89 & 1 & 2 & 1 & 5 & \# \\
\hline $\mathrm{H}$ & 1.07137 & 1 & 121.8788 & 1 & 179.0074 & 1 & 8 & 7 & 6 & \# \\
\hline $\mathrm{H}$ & 1.066719 & 1 & 128.3812 & 1 & 179.1662 & 1 & 9 & 8 & 7 & \# \\
\hline $\mathrm{H}$ & 1.072552 & 1 & 71.30419 & 1 & -36.4352 & 1 & 11 & 3 & 2 & $\#$ \\
\hline $\mathrm{H}$ & 1.074089 & 1 & 120.4149 & 1 & -94.6422 & 1 & 12 & 11 & 3 & \# \\
\hline $\mathrm{H}$ & 1.074151 & 1 & 118.6617 & 1 & -1.69397 & 1 & 17 & 13 & 12 & $\#$ \\
\hline $\mathrm{H}$ & 1.070959 & 1 & 121.4663 & 1 & -178.795 & 1 & 18 & 17 & 13 & \# \\
\hline $\mathrm{H}$ & 1.069778 & 1 & 118.8396 & 1 & 179.2406 & 1 & 20 & 19 & 18 & $\#$ \\
\hline $\mathrm{H}$ & 1.074119 & 1 & 118.9018 & 1 & 179.7527 & 1 & 25 & 24 & 23 & \# \\
\hline $\mathrm{H}$ & 1.071973 & 1 & 121.8246 & 1 & 178.8015 & 1 & 26 & 25 & 24 & $\#$ \\
\hline $\mathrm{H}$ & 1.069705 & 1 & 119.8629 & 1 & -0.25918 & 1 & 27 & 23 & 22 & \# \\
\hline $\mathrm{H}$ & 1.070525 & 1 & 118.6003 & 1 & 179.6482 & 1 & 29 & 28 & 27 & $\#$ \\
\hline $\mathrm{H}$ & 1.074156 & 1 & 120.2685 & 1 & -179.967 & 1 & 30 & 29 & 28 & \# \\
\hline $\mathrm{H}$ & 1.082352 & 1 & 109.4552 & 1 & -125.099 & 1 & 31 & 3 & 2 & $\#$ \\
\hline $\mathrm{H}$ & 1.086616 & 1 & 107.7877 & 1 & -10.1765 & 1 & 31 & 3 & 2 & \# \\
\hline
\end{tabular}




\begin{tabular}{|c|c|c|c|c|c|c|c|c|c|c|}
\hline $\mathrm{H}$ & 1.012733 & 1 & 117.3566 & 1 & -95.2082 & 1 & 34 & 19 & 18 & \# \\
\hline $\mathrm{H}$ & 1.011984 & 1 & 116.941 & 1 & 74.6506 & 1 & 35 & 28 & 27 & \# \\
\hline $\mathrm{H}$ & 1.018463 & 1 & 113.4591 & 1 & 2.421113 & 1 & 38 & 36 & 34 & \# \\
\hline $\mathrm{H}$ & 1.01563 & 1 & 114.0607 & 1 & 5.216773 & 1 & 40 & 37 & 35 & \# \\
\hline $\mathrm{H}$ & 1.076233 & 1 & 119.4641 & 1 & 1.411124 & 1 & 44 & 42 & 38 & \# \\
\hline $\mathrm{H}$ & 1.071324 & 1 & 118.5698 & 1 & 179.7737 & 1 & 45 & 44 & 42 & \# \\
\hline $\mathrm{H}$ & 1.071915 & 1 & 118.7863 & 1 & 179.4182 & 1 & 47 & 46 & 45 & \# \\
\hline $\mathrm{H}$ & 1.066375 & 1 & 119.8338 & 1 & 179.918 & 1 & 48 & 47 & 46 & \# \\
\hline $\mathrm{H}$ & 1.075607 & 1 & 119.1085 & 1 & 1.226081 & 1 & 49 & 43 & 40 & \# \\
\hline $\mathrm{H}$ & 1.071529 & 1 & 120.7981 & 1 & 179.874 & 1 & 50 & 49 & 43 & \# \\
\hline $\mathrm{H}$ & 1.071718 & 1 & 121.3672 & 1 & 179.3572 & 1 & 52 & 51 & 50 & \# \\
\hline $\mathrm{H}$ & 1.066848 & 1 & 119.9975 & 1 & -3.35863 & 1 & 53 & 43 & 40 & \# \\
\hline $\mathrm{H}$ & 1.081708 & 1 & 109.7219 & 1 & -13.2234 & 1 & 54 & 32 & 16 & \# \\
\hline $\mathrm{H}$ & 1.08471 & 1 & 108.1042 & 1 & 105.0905 & 1 & 54 & 32 & 16 & \# \\
\hline $\mathrm{H}$ & 1.078639 & 1 & 109.3467 & 1 & 53.51784 & 1 & 55 & 33 & 21 & \# \\
\hline $\mathrm{H}$ & 1.084346 & 1 & 109.2672 & 1 & -64.5959 & 1 & 55 & 33 & 21 & \# \\
\hline $\mathrm{H}$ & 1.084114 & 1 & 108.0967 & 1 & -51.6314 & 1 & 56 & 54 & 32 & \# \\
\hline $\mathrm{H}$ & 1.079206 & 1 & 108.524 & 1 & 64.64596 & 1 & 56 & 54 & 32 & \# \\
\hline $\mathrm{H}$ & 1.082139 & 1 & 107.9513 & 1 & -40.1728 & 1 & 57 & 55 & 33 & \# \\
\hline $\mathrm{H}$ & 1.085975 & 1 & 108.0915 & 1 & -155.637 & 1 & 57 & 55 & 33 & \# \\
\hline $\mathrm{H}$ & 1.085875 & 1 & 107.5283 & 1 & 161.2798 & 1 & 58 & 7 & 6 & \# \\
\hline $\mathrm{H}$ & 1.082684 & 1 & 109.3413 & 1 & 46.63695 & 1 & 58 & 7 & 6 & \# \\
\hline $\mathrm{C}$ & 2.439747 & 1 & 146.3765 & 1 & -179.517 & 1 & 51 & 50 & 49 & $\#$ \\
\hline $\mathrm{H}$ & 1.078284 & 1 & 135.2989 & 1 & 0.560588 & 1 & 97 & 51 & 50 & \# \\
\hline $\mathrm{H}$ & 1.083643 & 1 & 95.43336 & 1 & 125.4394 & 1 & 97 & 51 & 50 & \# \\
\hline $\mathrm{H}$ & 1.08373 & 1 & 95.74989 & 1 & -124.664 & 1 & 97 & 51 & 50 & \# \\
\hline $\mathrm{C}$ & 2.439338 & 1 & 93.75366 & 1 & -179.956 & 1 & 46 & 45 & 44 & \# \\
\hline $\mathrm{H}$ & 1.07826 & 1 & 135.2994 & 1 & 179.9568 & 1 & 101 & 46 & 45 & \# \\
\hline $\mathrm{H}$ & 1.083284 & 1 & 95.42293 & 1 & -55.1196 & 1 & 101 & 46 & 45 & \# \\
\hline $\mathrm{H}$ & 1.083351 & 1 & 95.64003 & 1 & 54.7809 & 1 & 101 & 46 & 45 & \# \\
\hline $\mathrm{O}$ & 1.389256 & 1 & 116.3123 & 1 & -179.477 & 1 & 51 & 50 & 49 & \# \\
\hline $\mathrm{O}$ & 1.388883 & 1 & 123.8962 & 1 & -179.714 & 1 & 46 & 45 & 44 & \# \\
\hline $\mathrm{C}$ & 6.095288 & 1 & 111.4628 & 1 & -8.45064 & 1 & 44 & 42 & 38 & \# \\
\hline $\mathrm{C}$ & 1.39082 & 1 & 57.63383 & 1 & -4.91703 & 1 & 107 & 44 & 42 & \# \\
\hline $\mathrm{C}$ & 1.392069 & 1 & 119.3198 & 1 & -176.507 & 1 & 108 & 107 & 44 & \# \\
\hline $\mathrm{C}$ & 1.391357 & 1 & 120.6605 & 1 & 0.190369 & 1 & 109 & 108 & 107 & \# \\
\hline $\mathrm{C}$ & 1.391069 & 1 & 119.2671 & 1 & -0.0084 & 1 & 110 & 109 & 108 & \# \\
\hline $\mathrm{C}$ & 1.386312 & 1 & 176.2884 & 1 & -63.7179 & 1 & 107 & 44 & 42 & \# \\
\hline
\end{tabular}




$\begin{array}{rrrrrrrrrrr}\mathrm{H} & 1.071482 & 1 & 60.64073 & 1 & 171.4574 & 1 & 107 & 44 & 42 & \# \\ \mathrm{H} & 1.069342 & 1 & 119.7453 & 1 & -179.94 & 1 & 109 & 108 & 107 & \# \\ \mathrm{H} & 1.071347 & 1 & 118.1128 & 1 & 179.7993 & 1 & 111 & 110 & 109 & \# \\ \mathrm{H} & 1.075105 & 1 & 120.0487 & 1 & -123.035 & 1 & 112 & 107 & 44 & \# \\ \mathrm{C} & 1.505342 & 1 & 118.9837 & 1 & 3.342883 & 1 & 108 & 107 & 44 & \# \\ \mathrm{C} & 1.506957 & 1 & 121.8318 & 1 & 179.8351 & 1 & 110 & 109 & 108 & \# \\ \mathrm{O} & 1.255403 & 1 & 117.7413 & 1 & -177.772 & 1 & 118 & 110 & 109 & \# \\ \mathrm{O} & 1.277332 & 1 & 117.9152 & 1 & 2.510659 & 1 & 118 & 110 & 109 & \# \\ \mathrm{O} & 1.278834 & 1 & 117.5712 & 1 & -179.044 & 1 & 117 & 108 & 107 & \# \\ \mathrm{O} & 1.254623 & 1 & 118.2362 & 1 & 0.599685 & 1 & 117 & 108 & 107 & \# \\ & 0 & 0 & 0 & 0 & 0 & 0 & 0 & 0 & 0 & 0\end{array}$

Total energy $=-4629.841368$ Hartree 
M analogs; $l=3$ with terephthalate

Z-matrices

\begin{tabular}{|c|c|c|c|c|c|c|c|c|c|}
\hline $\mathrm{C}$ & 0 & 1 & 0 & 1 & 0 & 1 & 0 & 0 & 0 \\
\hline $\mathrm{C}$ & 1.339316 & 1 & 0 & 1 & 0 & 1 & 1 & 0 & 0 \\
\hline $\mathrm{C}$ & 1.4496 & 1 & 115.0703 & 1 & 0 & 1 & 2 & 1 & 0 \\
\hline $\mathrm{C}$ & 1.355589 & 1 & 112.8612 & 1 & -0.57616 & 1 & 3 & 2 & 1 \\
\hline$S$ & 1.780036 & 1 & 111.4105 & 1 & -0.2852 & 1 & 1 & 2 & 3 \\
\hline $\mathrm{C}$ & 1.460199 & 1 & 130.1306 & 1 & 178.4526 & 1 & 4 & 3 & 2 \\
\hline $\mathrm{C}$ & 1.353786 & 1 & 130.5863 & 1 & 127.2589 & 1 & 6 & 4 & 3 \\
\hline $\mathrm{C}$ & 1.449753 & 1 & 112.7772 & 1 & 179.8718 & 1 & 7 & 6 & 4 \\
\hline $\mathrm{C}$ & 1.338797 & 1 & 115.1105 & 1 & -0.65175 & 1 & 8 & 7 & 6 \\
\hline$S$ & 1.779597 & 1 & 111.42 & 1 & -0.17403 & 1 & 9 & 8 & 7 \\
\hline $\mathrm{C}$ & 5.69187 & 1 & 89.16369 & 1 & -108.291 & 1 & 3 & 2 & 1 \\
\hline $\mathrm{C}$ & 1.357518 & 1 & 131.8985 & 1 & 82.81652 & 1 & 11 & 3 & 2 \\
\hline $\mathrm{C}$ & 1.416103 & 1 & 120.5299 & 1 & 80.72378 & 1 & 12 & 11 & 3 \\
\hline $\mathrm{C}$ & 1.414192 & 1 & 119.3257 & 1 & 2.761023 & 1 & 13 & 12 & 11 \\
\hline $\mathrm{C}$ & 1.435669 & 1 & 119.5284 & 1 & -1.91501 & 1 & 14 & 13 & 12 \\
\hline $\mathrm{C}$ & 1.366814 & 1 & 118.5494 & 1 & -1.15721 & 1 & 15 & 14 & 13 \\
\hline $\mathrm{C}$ & 1.418792 & 1 & 121.225 & 1 & -175.773 & 1 & 13 & 12 & 11 \\
\hline $\mathrm{C}$ & 1.357613 & 1 & 120.9784 & 1 & 176.4808 & 1 & 17 & 13 & 12 \\
\hline $\mathrm{C}$ & 1.415163 & 1 & 120.0117 & 1 & 1.347279 & 1 & 18 & 17 & 13 \\
\hline $\mathrm{C}$ & 1.362105 & 1 & 120.2904 & 1 & 1.36635 & 1 & 19 & 18 & 17 \\
\hline $\mathrm{C}$ & 2.484892 & 1 & 117.6852 & 1 & -147.127 & 1 & 15 & 14 & 13 \\
\hline $\mathrm{C}$ & 1.367846 & 1 & 31.387 & 1 & -104.986 & 1 & 21 & 15 & 14 \\
\hline $\mathrm{C}$ & 1.434916 & 1 & 118.7121 & 1 & -178.469 & 1 & 22 & 21 & 15 \\
\hline $\mathrm{C}$ & 1.413504 & 1 & 119.2546 & 1 & -1.72304 & 1 & 23 & 22 & 21 \\
\hline $\mathrm{C}$ & 1.416514 & 1 & 119.5028 & 1 & -0.18851 & 1 & 24 & 23 & 22 \\
\hline $\mathrm{C}$ & 1.358331 & 1 & 120.6144 & 1 & 1.559125 & 1 & 25 & 24 & 23 \\
\hline $\mathrm{C}$ & 1.424276 & 1 & 122.5154 & 1 & 176.2504 & 1 & 23 & 22 & 21 \\
\hline $\mathrm{C}$ & 1.364928 & 1 & 121.3131 & 1 & -177.674 & 1 & 27 & 23 & 22 \\
\hline $\mathrm{C}$ & 1.417107 & 1 & 120.0198 & 1 & -1.05612 & 1 & 28 & 27 & 23 \\
\hline $\mathrm{C}$ & 1.357162 & 1 & 120.0035 & 1 & 0.885169 & 1 & 29 & 28 & 27 \\
\hline $\mathrm{C}$ & 1.516737 & 1 & 120.5517 & 1 & 178.6988 & 1 & 3 & 2 & 1 \\
\hline $\mathrm{O}$ & 1.382096 & 1 & 119.9654 & 1 & -177.223 & 1 & 16 & 15 & 14 \\
\hline $\mathrm{O}$ & 1.386805 & 1 & 89.48731 & 1 & 80.48877 & 1 & 21 & 15 & 14 \\
\hline $\mathrm{N}$ & 1.422797 & 1 & 118.6101 & 1 & 178.4944 & 1 & 19 & 18 & 17 \\
\hline $\mathrm{N}$ & 1.419228 & 1 & 119.7445 & 1 & -175.684 & 1 & 28 & 27 & 23 \\
\hline $\mathrm{C}$ & 1.346671 & 1 & 126.1837 & 1 & 84.41894 & 1 & 34 & 19 & 18 \\
\hline
\end{tabular}




\begin{tabular}{|c|c|c|c|c|c|c|c|c|c|c|}
\hline $\mathrm{C}$ & 1.350195 & 1 & 127.8394 & 1 & -123.664 & 1 & 35 & 28 & 27 & \# \\
\hline $\mathrm{N}$ & 1.339833 & 1 & 112.2484 & 1 & -174.415 & 1 & 36 & 34 & 19 & \# \\
\hline S & 1.766794 & 1 & 121.5255 & 1 & 4.683601 & 1 & 36 & 34 & 19 & \# \\
\hline $\mathrm{N}$ & 1.340673 & 1 & 111.9552 & 1 & -175.379 & 1 & 37 & 35 & 28 & \# \\
\hline S & 1.763867 & 1 & 121.7273 & 1 & 4.401406 & 1 & 37 & 35 & 28 & \# \\
\hline $\mathrm{C}$ & 1.408512 & 1 & 133.275 & 1 & 177.6044 & 1 & 38 & 36 & 34 & \# \\
\hline $\mathrm{C}$ & 1.408584 & 1 & 133.4829 & 1 & -179.823 & 1 & 40 & 37 & 35 & \# \\
\hline $\mathrm{C}$ & 1.393283 & 1 & 115.0759 & 1 & -159.707 & 1 & 42 & 38 & 36 & \# \\
\hline $\mathrm{C}$ & 1.386775 & 1 & 121.1956 & 1 & -177.939 & 1 & 44 & 42 & 38 & \# \\
\hline $\mathrm{C}$ & 1.384792 & 1 & 119.643 & 1 & -0.09967 & 1 & 45 & 44 & 42 & \# \\
\hline $\mathrm{C}$ & 1.384784 & 1 & 119.6616 & 1 & 0.445842 & 1 & 46 & 45 & 44 & \# \\
\hline $\mathrm{C}$ & 1.384782 & 1 & 120.7937 & 1 & -0.09022 & 1 & 47 & 46 & 45 & \# \\
\hline $\mathrm{C}$ & 1.404192 & 1 & 114.9404 & 1 & -165.772 & 1 & 43 & 40 & 37 & \# \\
\hline $\mathrm{C}$ & 1.377898 & 1 & 120.8135 & 1 & -178.628 & 1 & 49 & 43 & 40 & \# \\
\hline $\mathrm{C}$ & 1.389151 & 1 & 120.1338 & 1 & 0.004884 & 1 & 50 & 49 & 43 & \# \\
\hline $\mathrm{C}$ & 1.379912 & 1 & 119.666 & 1 & 0.417565 & 1 & 51 & 50 & 49 & \# \\
\hline $\mathrm{C}$ & 1.386104 & 1 & 126.4555 & 1 & 16.45174 & 1 & 43 & 40 & 37 & \# \\
\hline $\mathrm{C}$ & 1.445101 & 1 & 119.5452 & 1 & 117.8566 & 1 & 32 & 16 & 15 & \# \\
\hline $\mathrm{C}$ & 1.449849 & 1 & 121.4445 & 1 & -89.5883 & 1 & 33 & 21 & 15 & \# \\
\hline $\mathrm{C}$ & 1.518805 & 1 & 108.9363 & 1 & -122.288 & 1 & 54 & 32 & 16 & \# \\
\hline $\mathrm{C}$ & 1.519641 & 1 & 106.7935 & 1 & 171.9256 & 1 & 55 & 33 & 21 & \# \\
\hline $\mathrm{C}$ & 1.515218 & 1 & 127.3819 & 1 & 2.266555 & 1 & 7 & 6 & 4 & \# \\
\hline $\mathrm{H}$ & 1.066781 & 1 & 128.3967 & 1 & 178.9805 & 1 & 1 & 2 & 3 & \# \\
\hline $\mathrm{H}$ & 1.071738 & 1 & 123.0486 & 1 & -179.927 & 1 & 2 & 1 & 5 & \# \\
\hline $\mathrm{H}$ & 1.071393 & 1 & 121.8269 & 1 & 179.0629 & 1 & 8 & 7 & 6 & \# \\
\hline $\mathrm{H}$ & 1.066738 & 1 & 128.4005 & 1 & 179.2191 & 1 & 9 & 8 & 7 & \# \\
\hline $\mathrm{H}$ & 1.072494 & 1 & 75.98857 & 1 & -36.8319 & 1 & 11 & 3 & 2 & \# \\
\hline $\mathrm{H}$ & 1.073928 & 1 & 120.5048 & 1 & -98.6877 & 1 & 12 & 11 & 3 & \# \\
\hline $\mathrm{H}$ & 1.074 & 1 & 118.6628 & 1 & -2.37512 & 1 & 17 & 13 & 12 & \# \\
\hline $\mathrm{H}$ & 1.071229 & 1 & 121.4103 & 1 & -178.372 & 1 & 18 & 17 & 13 & \# \\
\hline $\mathrm{H}$ & 1.070318 & 1 & 119.0253 & 1 & 178.8258 & 1 & 20 & 19 & 18 & \# \\
\hline $\mathrm{H}$ & 1.074132 & 1 & 118.8697 & 1 & -179.802 & 1 & 25 & 24 & 23 & \# \\
\hline $\mathrm{H}$ & 1.072069 & 1 & 121.7936 & 1 & 177.3775 & 1 & 26 & 25 & 24 & \# \\
\hline $\mathrm{H}$ & 1.070425 & 1 & 119.6297 & 1 & 1.126708 & 1 & 27 & 23 & 22 & \# \\
\hline $\mathrm{H}$ & 1.069576 & 1 & 118.7565 & 1 & -179.602 & 1 & 29 & 28 & 27 & \# \\
\hline $\mathrm{H}$ & 1.07419 & 1 & 120.1311 & 1 & 179.8937 & 1 & 30 & 29 & 28 & \# \\
\hline $\mathrm{H}$ & 1.081342 & 1 & 109.556 & 1 & -129.704 & 1 & 31 & 3 & 2 & \# \\
\hline $\mathrm{H}$ & 1.086905 & 1 & 107.9227 & 1 & -14.6586 & 1 & 31 & 3 & 2 & \# \\
\hline
\end{tabular}




\begin{tabular}{|c|c|c|c|c|c|c|c|c|c|c|}
\hline $\mathrm{H}$ & 1.013949 & 1 & 117.6953 & 1 & -88.4913 & 1 & 34 & 19 & 18 & \# \\
\hline $\mathrm{H}$ & 1.01137 & 1 & 116.4551 & 1 & 60.94135 & 1 & 35 & 28 & 27 & \# \\
\hline $\mathrm{H}$ & 1.020524 & 1 & 113.8375 & 1 & 2.964044 & 1 & 38 & 36 & 34 & \# \\
\hline $\mathrm{H}$ & 1.022458 & 1 & 113.6979 & 1 & 3.67468 & 1 & 40 & 37 & 35 & \# \\
\hline $\mathrm{H}$ & 1.073976 & 1 & 119.02 & 1 & 1.508952 & 1 & 44 & 42 & 38 & \# \\
\hline $\mathrm{H}$ & 1.07119 & 1 & 118.7799 & 1 & 179.8818 & 1 & 45 & 44 & 42 & \# \\
\hline $\mathrm{H}$ & 1.071825 & 1 & 118.6703 & 1 & 179.5781 & 1 & 47 & 46 & 45 & \# \\
\hline $\mathrm{H}$ & 1.066129 & 1 & 119.9224 & 1 & -179.966 & 1 & 48 & 47 & 46 & \# \\
\hline $\mathrm{H}$ & 1.075067 & 1 & 119.492 & 1 & 0.895094 & 1 & 49 & 43 & 40 & \# \\
\hline $\mathrm{H}$ & 1.071346 & 1 & 120.7952 & 1 & 179.9958 & 1 & 50 & 49 & 43 & \# \\
\hline $\mathrm{H}$ & 1.07185 & 1 & 121.2733 & 1 & 179.6423 & 1 & 52 & 51 & 50 & \# \\
\hline $\mathrm{H}$ & 1.065707 & 1 & 120.0558 & 1 & -1.94658 & 1 & 53 & 43 & 40 & \# \\
\hline $\mathrm{H}$ & 1.082405 & 1 & 109.6762 & 1 & -0.39502 & 1 & 54 & 32 & 16 & \# \\
\hline $\mathrm{H}$ & 1.083481 & 1 & 107.1677 & 1 & 117.415 & 1 & 54 & 32 & 16 & \# \\
\hline $\mathrm{H}$ & 1.076961 & 1 & 109.5789 & 1 & 52.06951 & 1 & 55 & 33 & 21 & \# \\
\hline $\mathrm{H}$ & 1.083997 & 1 & 109.3349 & 1 & -66.2649 & 1 & 55 & 33 & 21 & \# \\
\hline $\mathrm{H}$ & 1.084052 & 1 & 108.0292 & 1 & -52.951 & 1 & 56 & 54 & 32 & \# \\
\hline $\mathrm{H}$ & 1.079241 & 1 & 108.9008 & 1 & 63.5646 & 1 & 56 & 54 & 32 & \# \\
\hline $\mathrm{H}$ & 1.082265 & 1 & 107.6559 & 1 & -37.3158 & 1 & 57 & 55 & 33 & \# \\
\hline $\mathrm{H}$ & 1.085468 & 1 & 108.1954 & 1 & -152.814 & 1 & 57 & 55 & 33 & \# \\
\hline $\mathrm{H}$ & 1.085858 & 1 & 107.3572 & 1 & 160.1213 & 1 & 58 & 7 & 6 & \# \\
\hline $\mathrm{H}$ & 1.082523 & 1 & 109.4927 & 1 & 45.4925 & 1 & 58 & 7 & 6 & \# \\
\hline $\mathrm{C}$ & 2.439208 & 1 & 146.3567 & 1 & -179.901 & 1 & 51 & 50 & 49 & \# \\
\hline $\mathrm{H}$ & 1.078141 & 1 & 135.2952 & 1 & 0.423966 & 1 & 97 & 51 & 50 & \# \\
\hline $\mathrm{H}$ & 1.083672 & 1 & 95.53484 & 1 & 125.4301 & 1 & 97 & 51 & 50 & \# \\
\hline $\mathrm{H}$ & 1.083704 & 1 & 95.61321 & 1 & -124.662 & 1 & 97 & 51 & 50 & \# \\
\hline $\mathrm{C}$ & 2.439803 & 1 & 93.81669 & 1 & 179.968 & 1 & 46 & 45 & 44 & \# \\
\hline $\mathrm{H}$ & 1.077974 & 1 & 135.2069 & 1 & 179.9739 & 1 & 101 & 46 & 45 & \# \\
\hline $\mathrm{H}$ & 1.08328 & 1 & 95.4424 & 1 & -55.1571 & 1 & 101 & 46 & 45 & \# \\
\hline $\mathrm{H}$ & 1.083334 & 1 & 95.68373 & 1 & 54.8191 & 1 & 101 & 46 & 45 & \# \\
\hline $\mathrm{O}$ & 1.388733 & 1 & 116.2739 & 1 & -179.73 & 1 & 51 & 50 & 49 & \# \\
\hline $\mathrm{O}$ & 1.387604 & 1 & 123.933 & 1 & -179.776 & 1 & 46 & 45 & 44 & \# \\
\hline $\mathrm{C}$ & 3.855874 & 1 & 93.31174 & 1 & -102.994 & 1 & 34 & 19 & 18 & \# \\
\hline $\mathrm{C}$ & 1.389653 & 1 & 98.75759 & 1 & 132.8299 & 1 & 107 & 34 & 19 & \# \\
\hline $\mathrm{C}$ & 1.391271 & 1 & 118.7735 & 1 & -149.042 & 1 & 108 & 107 & 34 & \# \\
\hline $\mathrm{C}$ & 1.388129 & 1 & 120.4912 & 1 & 1.380258 & 1 & 109 & 108 & 107 & \# \\
\hline $\mathrm{C}$ & 1.391603 & 1 & 120.5378 & 1 & -0.09993 & 1 & 110 & 109 & 108 & \# \\
\hline $\mathrm{C}$ & 1.38276 & 1 & 129.7151 & 1 & -10.2937 & 1 & 107 & 34 & 19 & \# \\
\hline
\end{tabular}




$\begin{array}{rrrrrrrrrrr}\mathrm{H} & 1.070026 & 1 & 36.77964 & 1 & -99.1399 & 1 & 107 & 34 & 19 & \# \\ \mathrm{H} & 1.07208 & 1 & 118.4874 & 1 & -179.068 & 1 & 109 & 108 & 107 & \# \\ \mathrm{H} & 1.069071 & 1 & 120.1174 & 1 & -42.8702 & 1 & 112 & 107 & 34 & \# \\ \mathrm{C} & 1.509512 & 1 & 119.9971 & 1 & 29.9276 & 1 & 108 & 107 & 34 & \# \\ \mathrm{C} & 1.508674 & 1 & 121.0593 & 1 & 178.9648 & 1 & 111 & 110 & 109 & \# \\ \mathrm{O} & 1.246695 & 1 & 119.2774 & 1 & 5.992398 & 1 & 117 & 111 & 110 & \# \\ \mathrm{O} & 1.283999 & 1 & 115.8229 & 1 & -174.053 & 1 & 117 & 111 & 110 & \# \\ \mathrm{O} & 1.287448 & 1 & 115.1077 & 1 & -12.9298 & 1 & 116 & 108 & 107 & \# \\ \mathrm{O} & 1.243509 & 1 & 119.4994 & 1 & 167.8826 & 1 & 116 & 108 & 107 & \# \\ \mathrm{H} & 1.071908 & 1 & 121.0586 & 1 & 179.5387 & 1 & 110 & 109 & 108 & \# \\ & 0 & 0 & 0 & 0 & 0 & 0 & 0 & 0 & 0 & 0\end{array}$

Total energy $=-4629.834405$ Hartree 


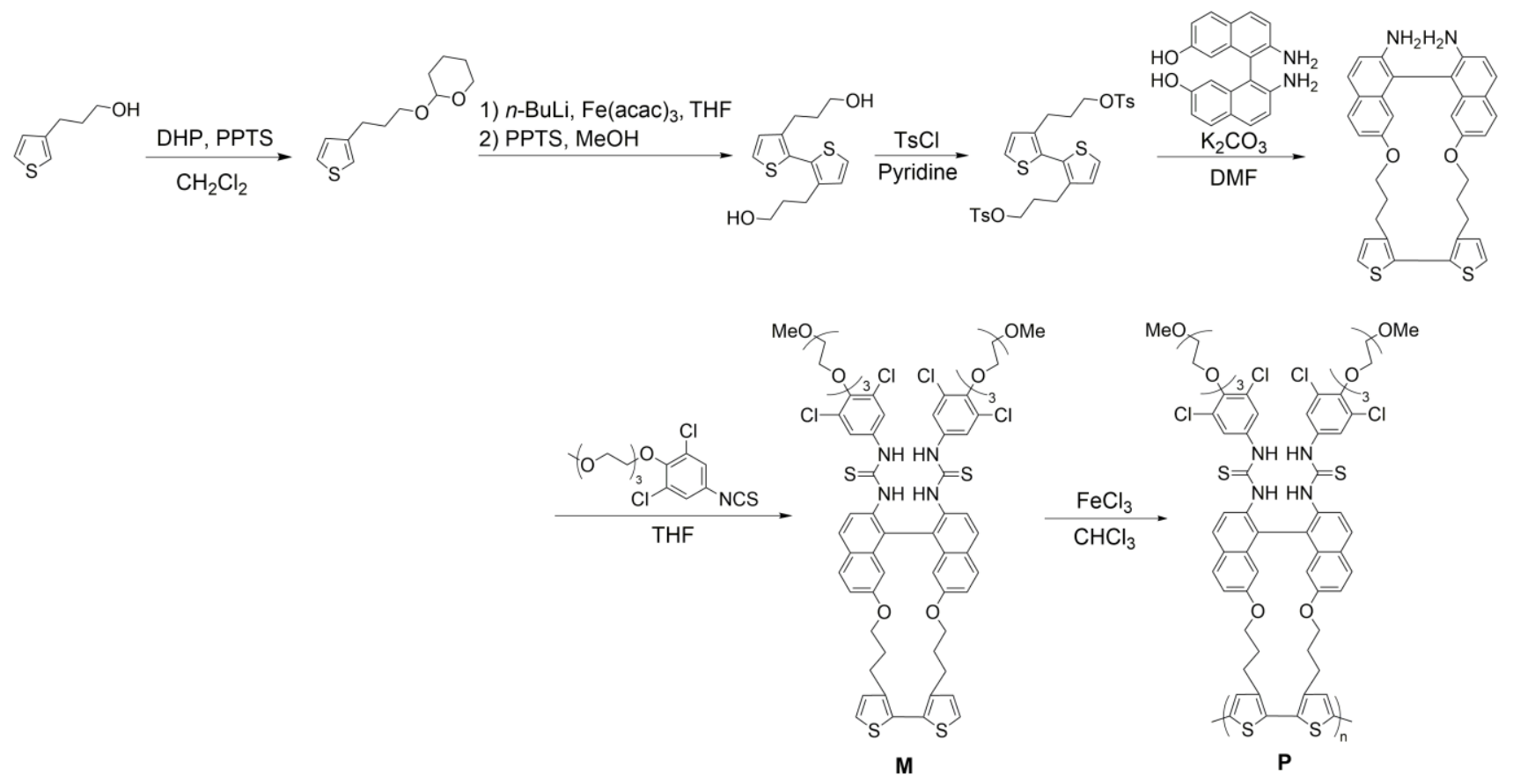

Figure S3. Synthetic scheme for $\mathbf{M}$ and polythiophene $(\mathbf{P})$ chemosensors. 

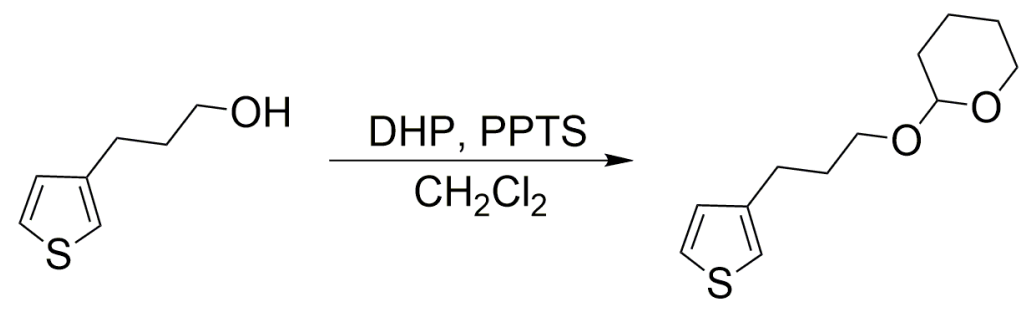

(a)

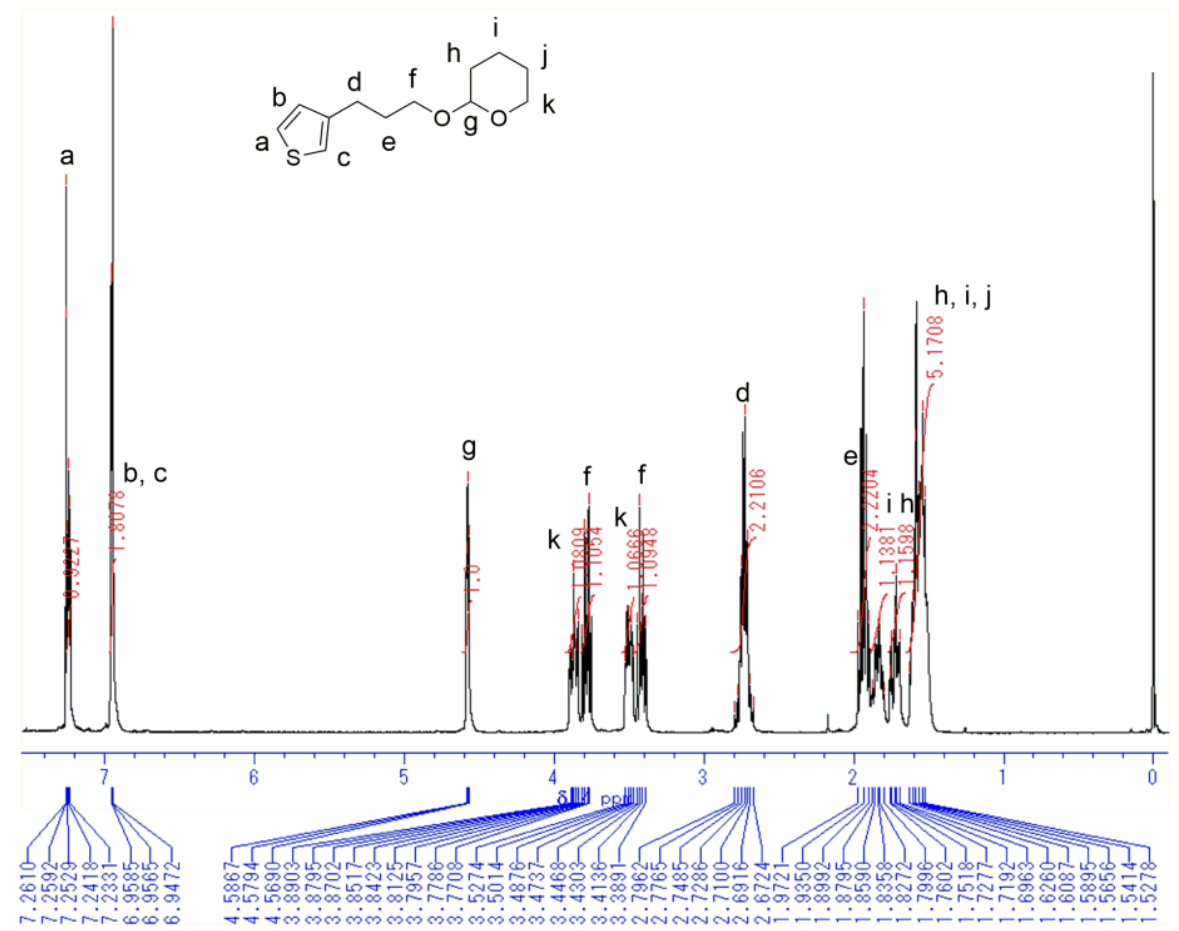

(b)

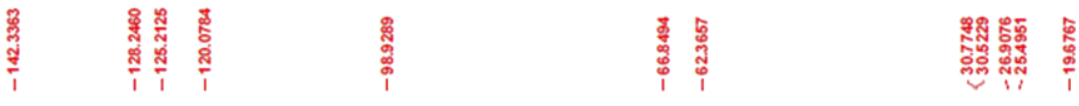

$$
\begin{aligned}
& \overbrace{5}^{3} \overbrace{6}^{7} \overbrace{8}^{10}
\end{aligned}
$$

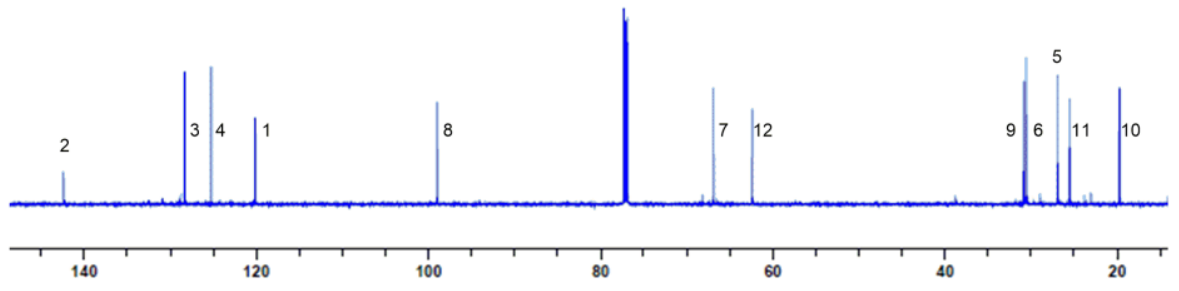

Figure S4. (a) ${ }^{1} \mathrm{H} \quad(400 \quad \mathrm{MHz})$ and $\quad$ (b) ${ }^{13} \mathrm{C} \quad \mathrm{NMR} \quad$ spectra $\quad\left(\begin{array}{llll}100 & \mathrm{MHz}\end{array}\right)$ of 3-(3-(2-tetrahydropyranyloxy)propyl)thiophene in $\mathrm{CDCl}_{3}$. 

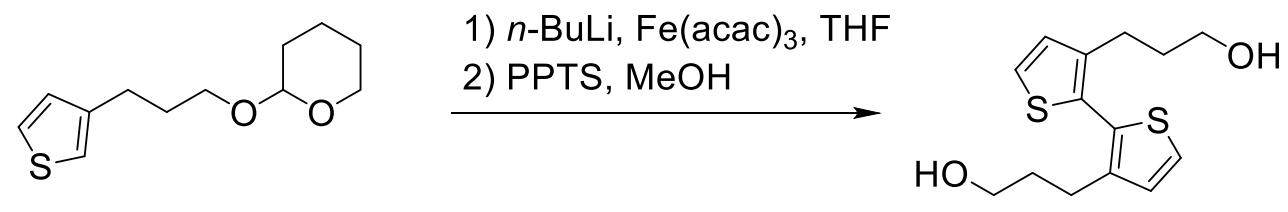

(a)

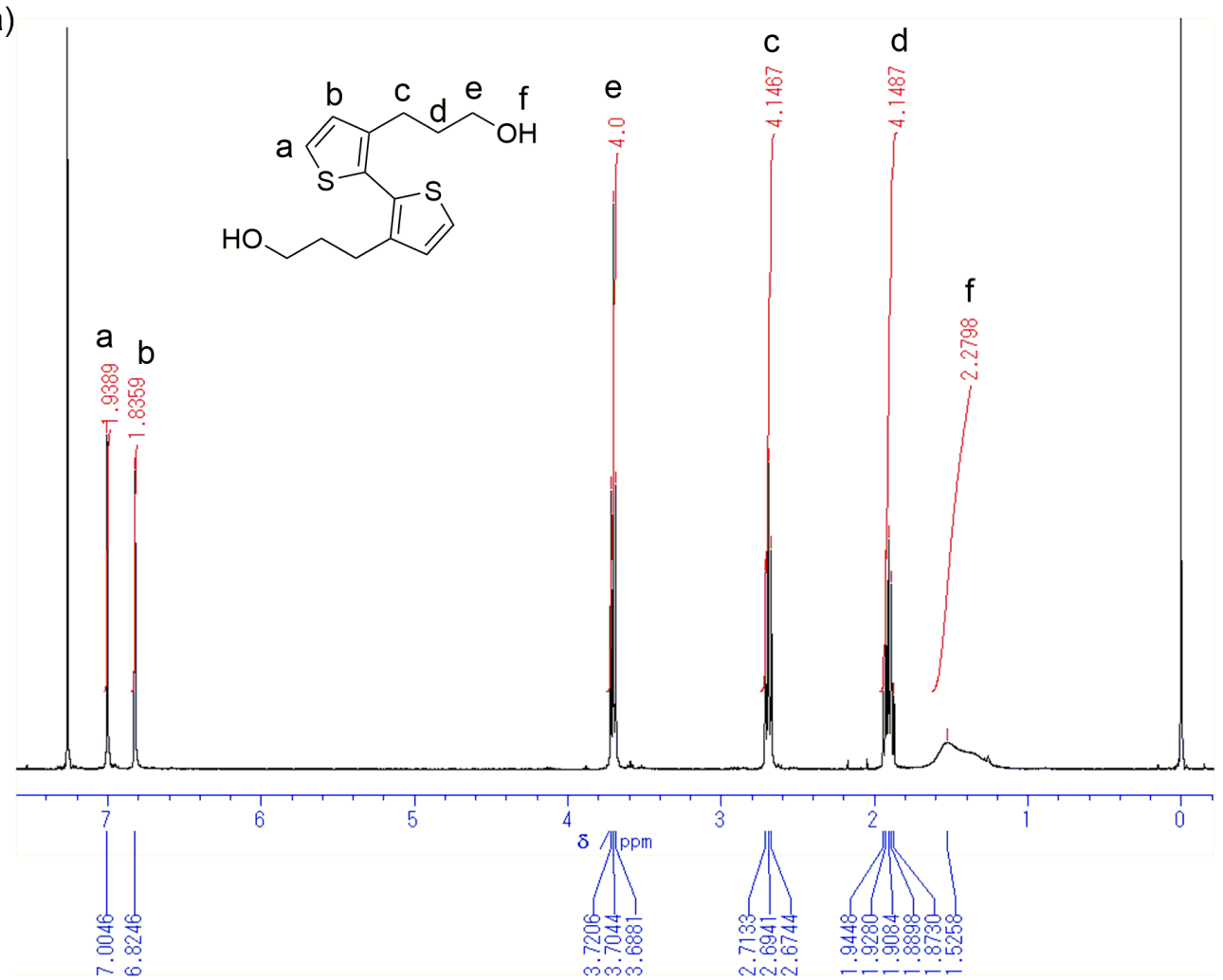

(b)

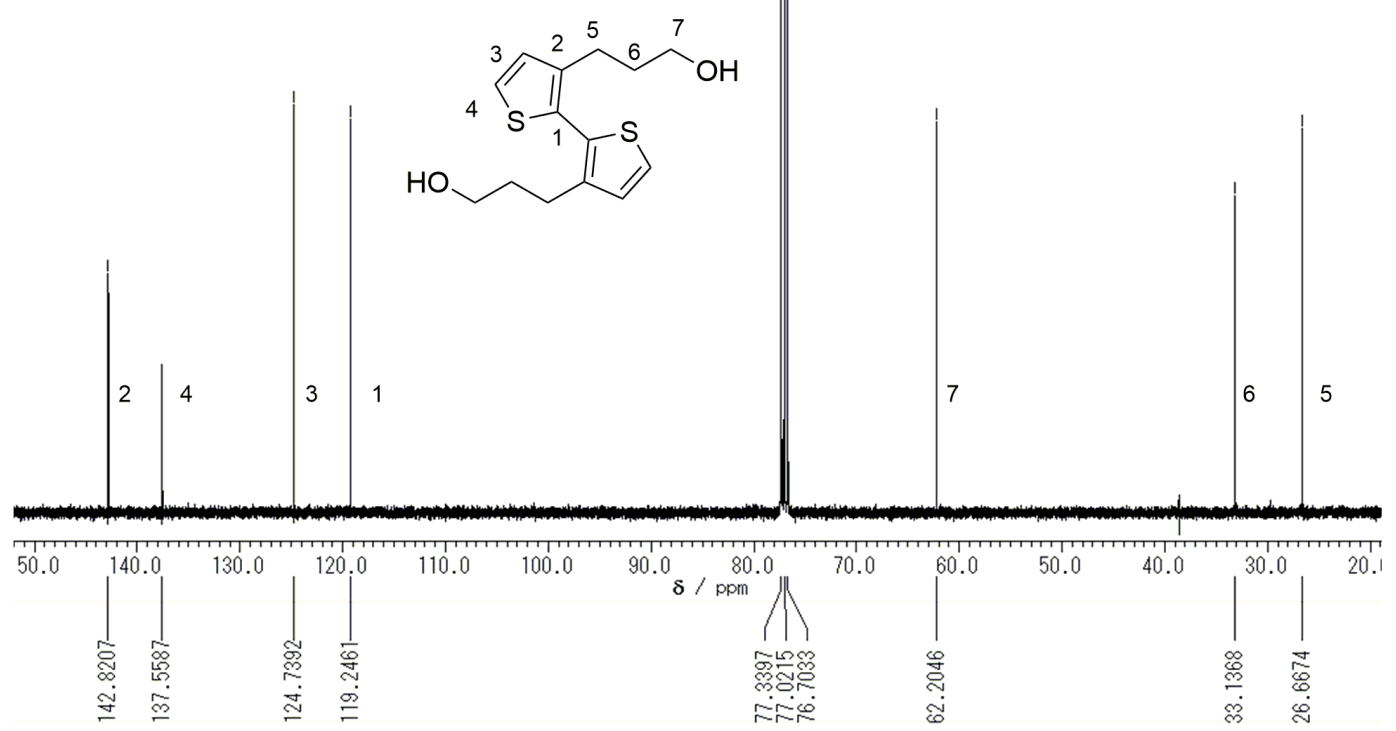

Figure S5. (a) ${ }^{1} \mathrm{H} \quad(400 \quad \mathrm{MHz})$ and $\quad$ (b) ${ }^{13} \mathrm{C} \quad \mathrm{NMR} \quad$ spectra $\quad\left(\begin{array}{lllll}100 & \mathrm{MHz}\end{array}\right)$ of 3,3'-(dihydroxypropyl)bithiophene in $\mathrm{CDCl}_{3}$. 
<smiles>OCCCc1ccsc1-c1sccc1CCCO[AsH2]OCCCc1ccsc1-c1sccc1CCC[OH2+]</smiles>

(a)

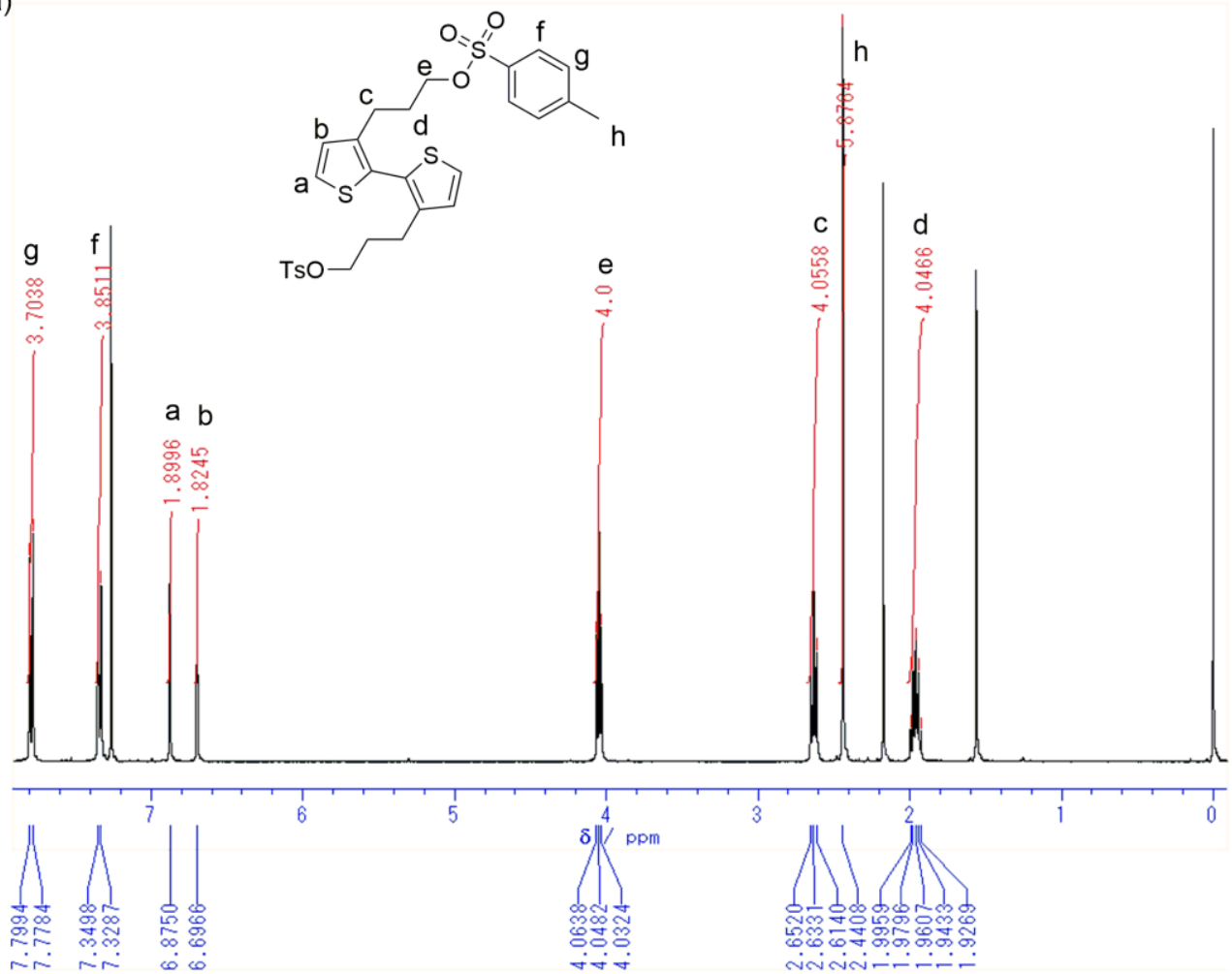

(b)

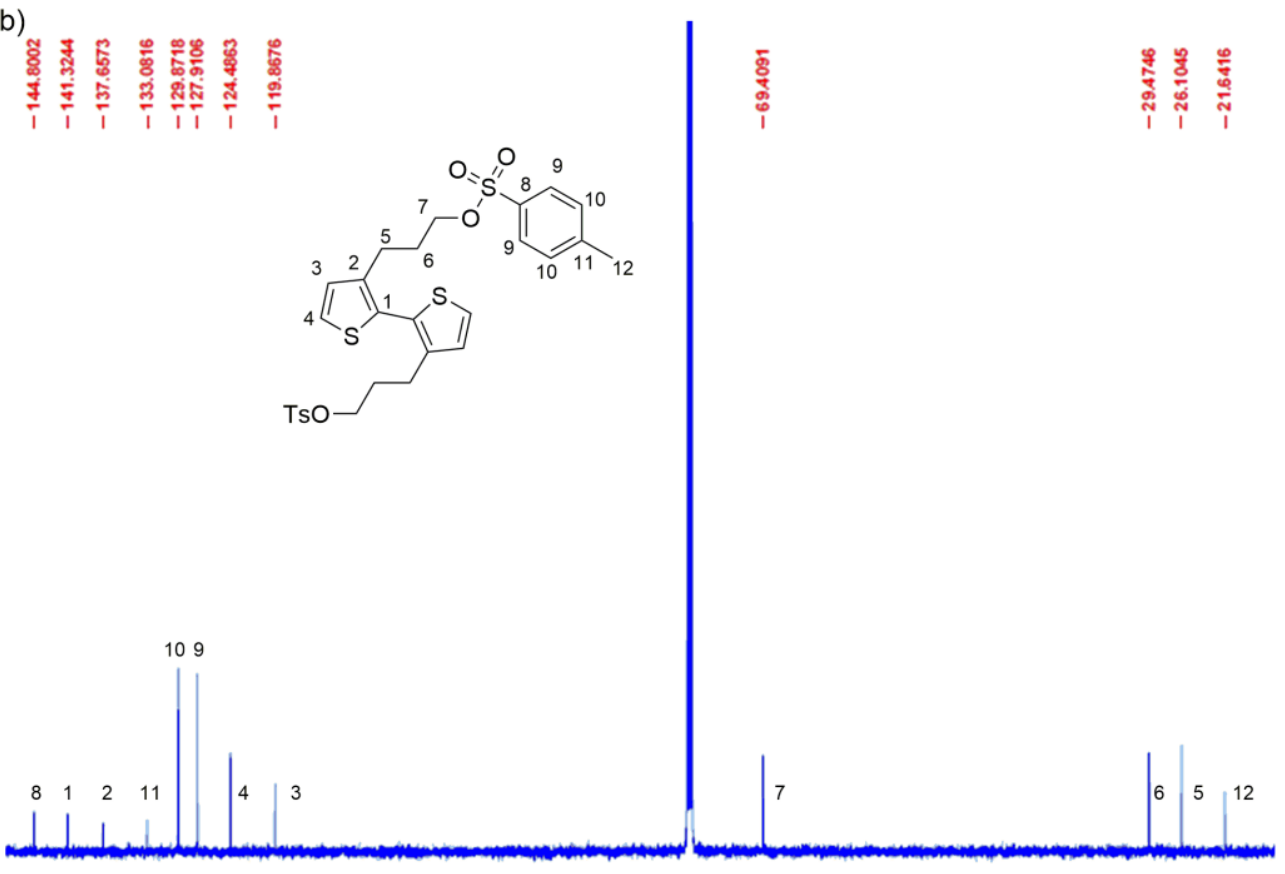

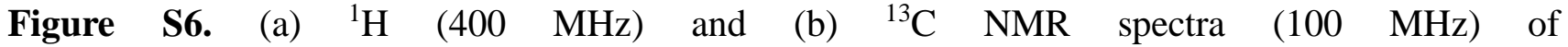
3,3'-(ditosylpropyl)bithiophene in $\mathrm{CDCl}_{3}$. 
<smiles></smiles>

(a)

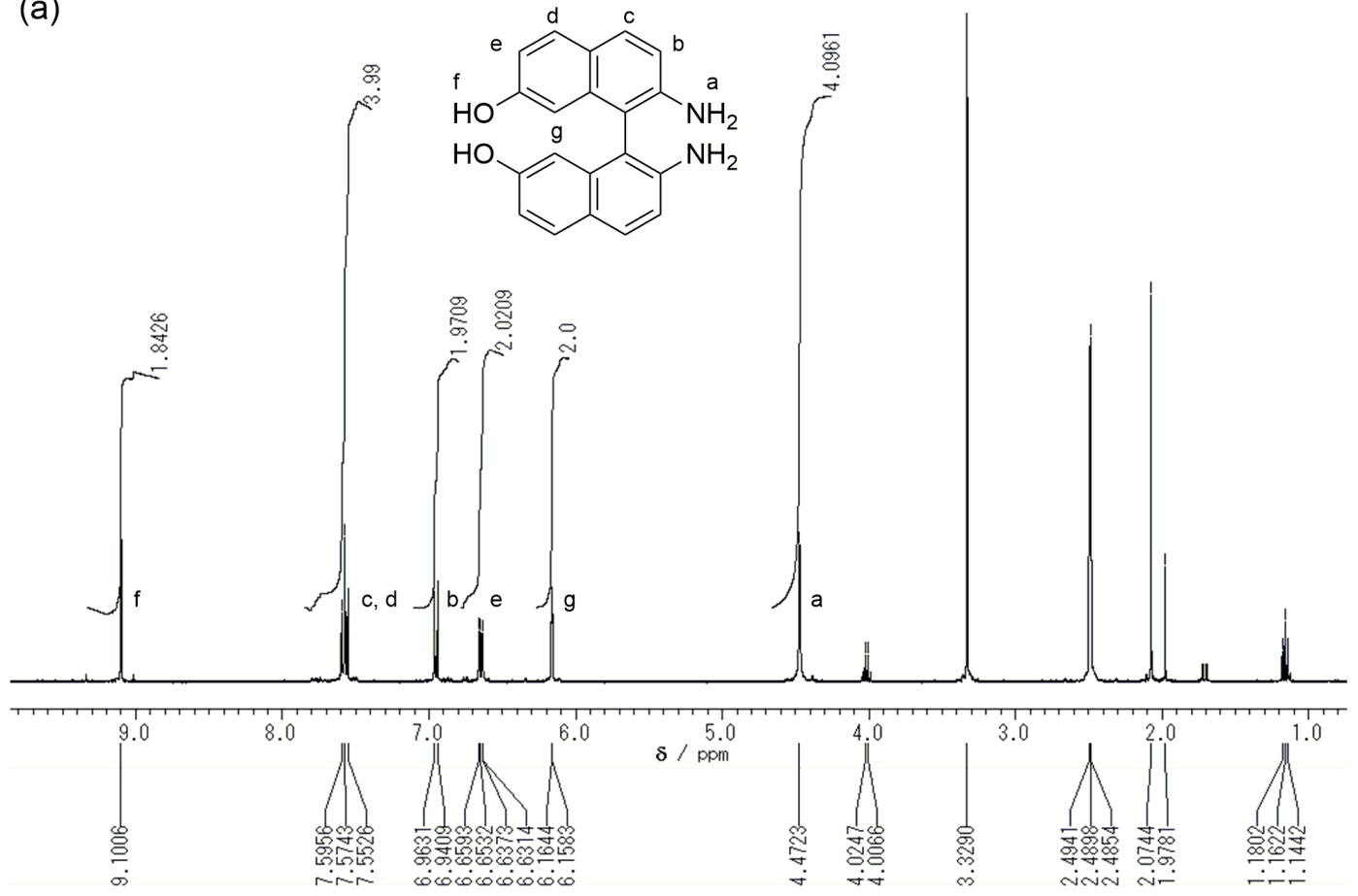

(b)
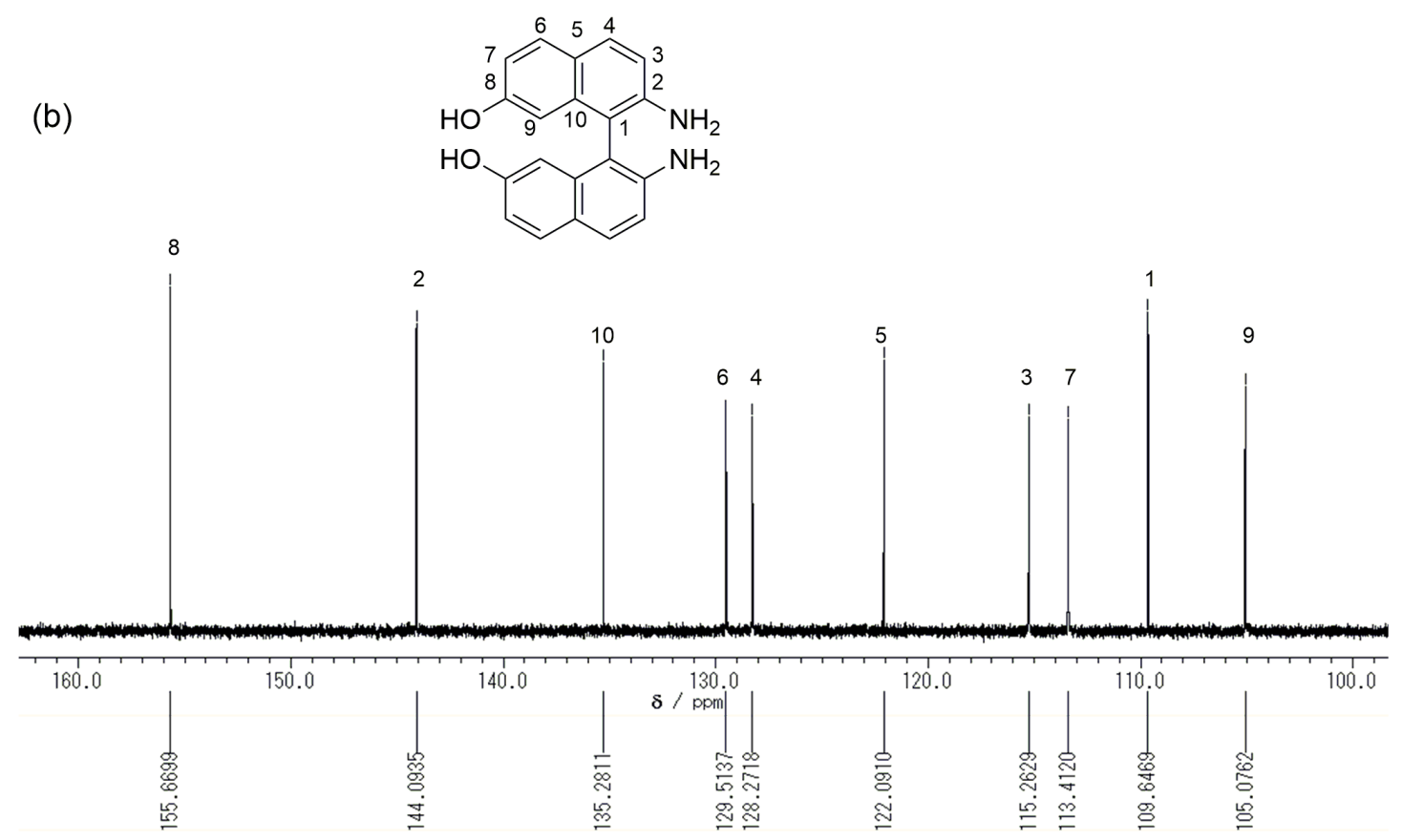

Figure S7. (a) ${ }^{1} \mathrm{H} \quad(400 \quad \mathrm{MHz})$ and $\quad$ (b) ${ }^{13} \mathrm{C} \quad \mathrm{NMR} \quad$ spectra $\quad\left(\begin{array}{lllll}100 & \mathrm{MHz}\end{array}\right)$ of 2,2'-diamino-[1,1'-binaphthalene]-7,7'-diol in DMSO- $d_{6}$. 


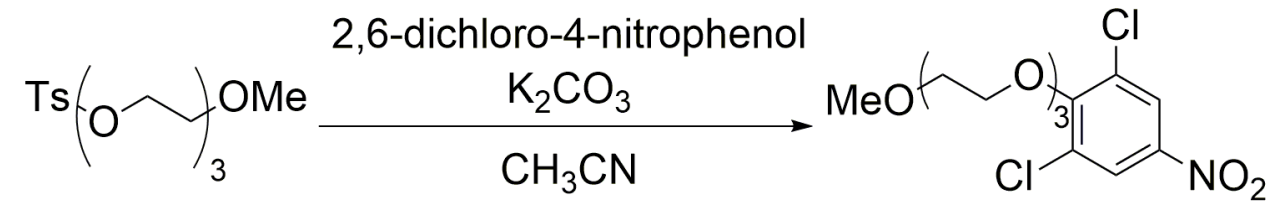

(a)
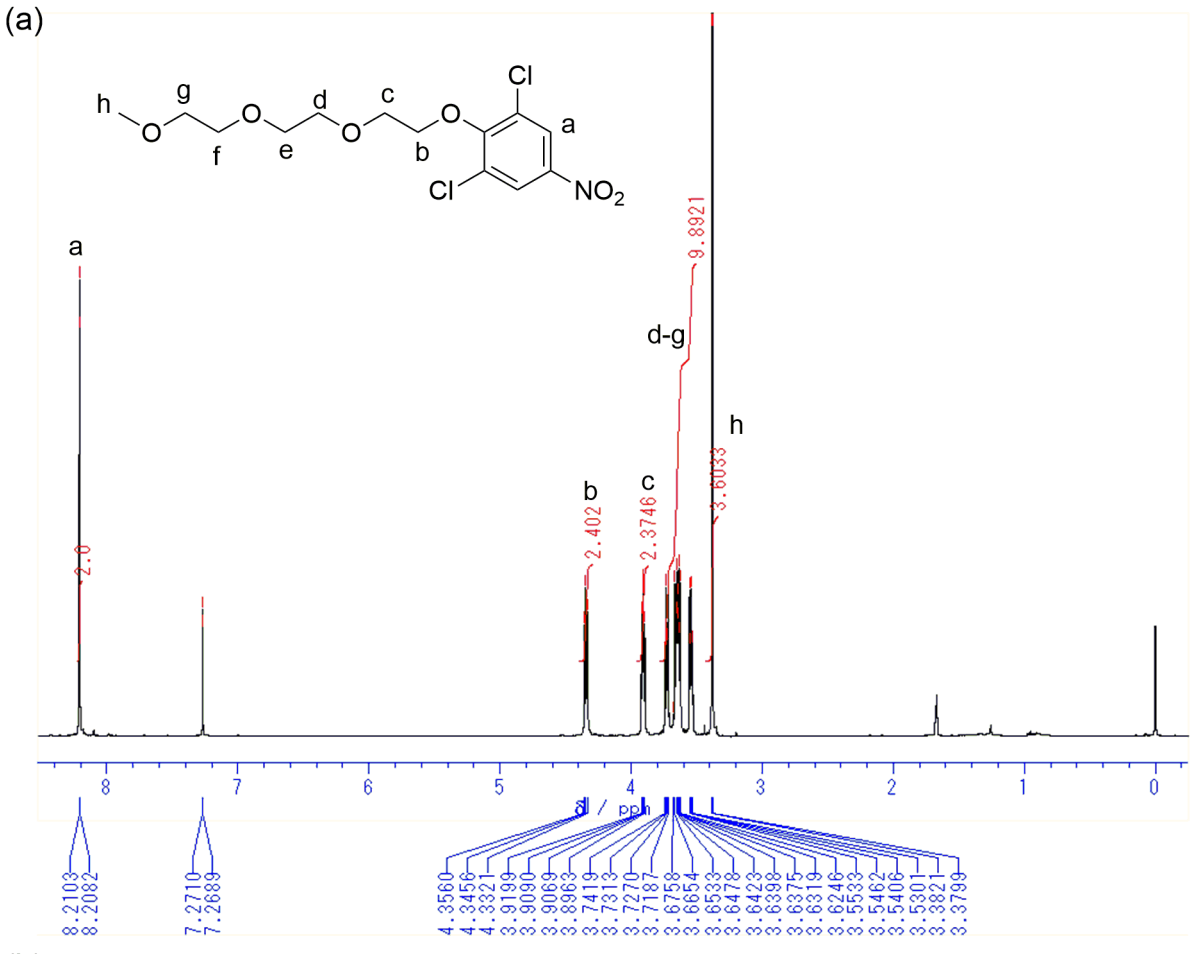

(b)
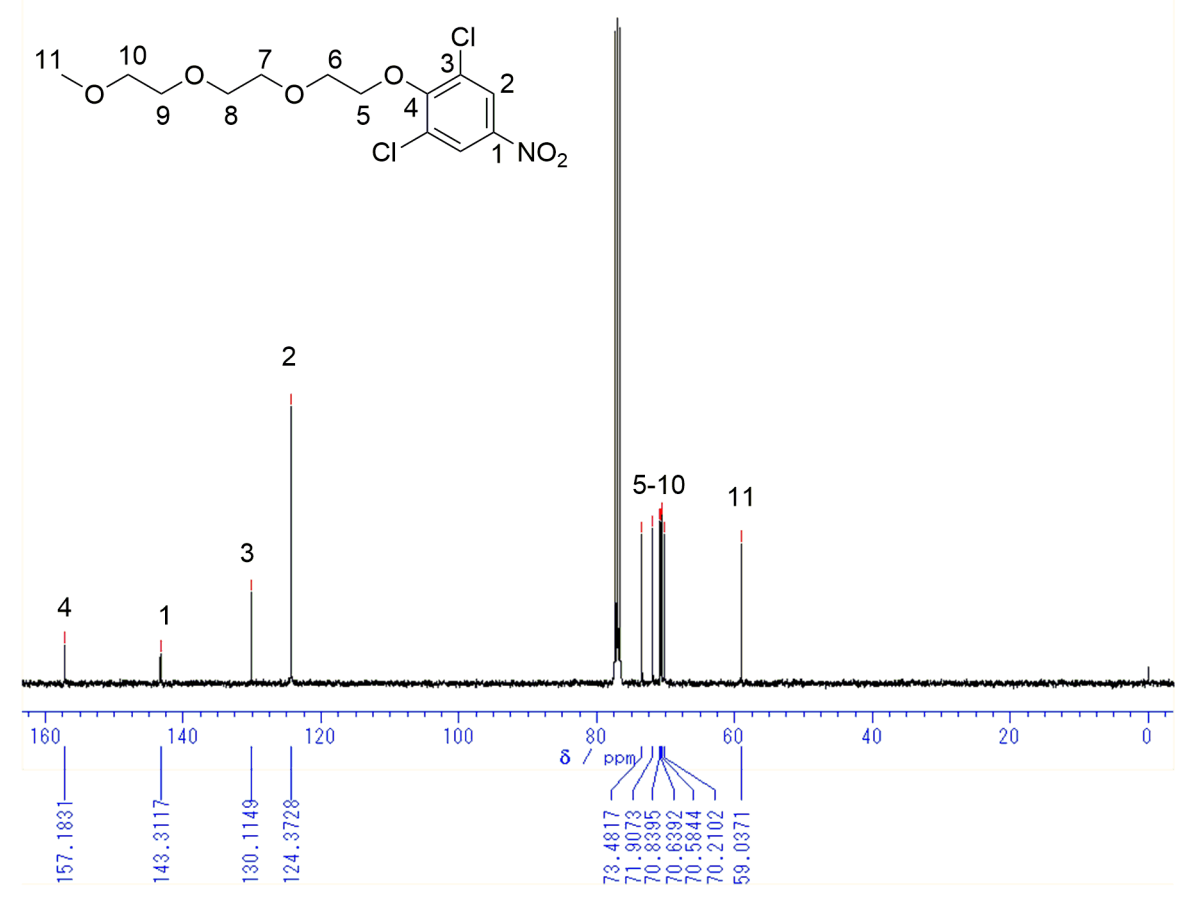

Figure S8. (a) ${ }^{1} \mathrm{H} \quad\left(\begin{array}{llllllll}400 & \mathrm{MHz}) & \text { and } & \text { (b) }{ }^{13} \mathrm{C} & \mathrm{NMR} & \text { spectra } & (100 & \mathrm{MHz}\end{array}\right)$ of 1-(2-(2-(2-methoxy)ethoxy)ethoxy)ethoxy-2,6-dichloro-4-nitrobenzene in $\mathrm{CDCl}_{3}$. 
<smiles>COCCOc1c(Cl)cc(N)cc1Cl</smiles>

(a)

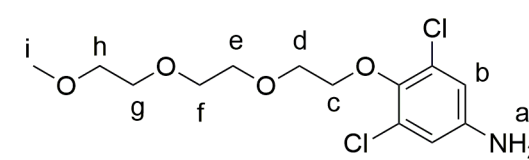

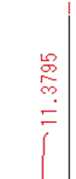

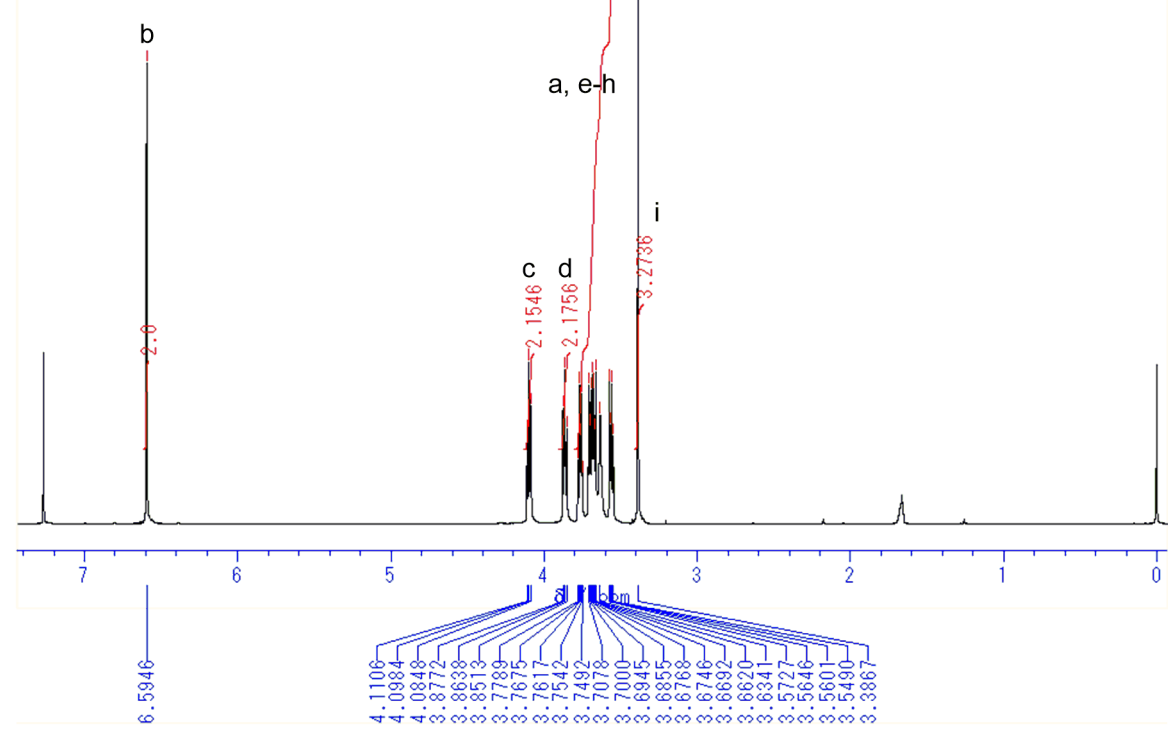

(b)
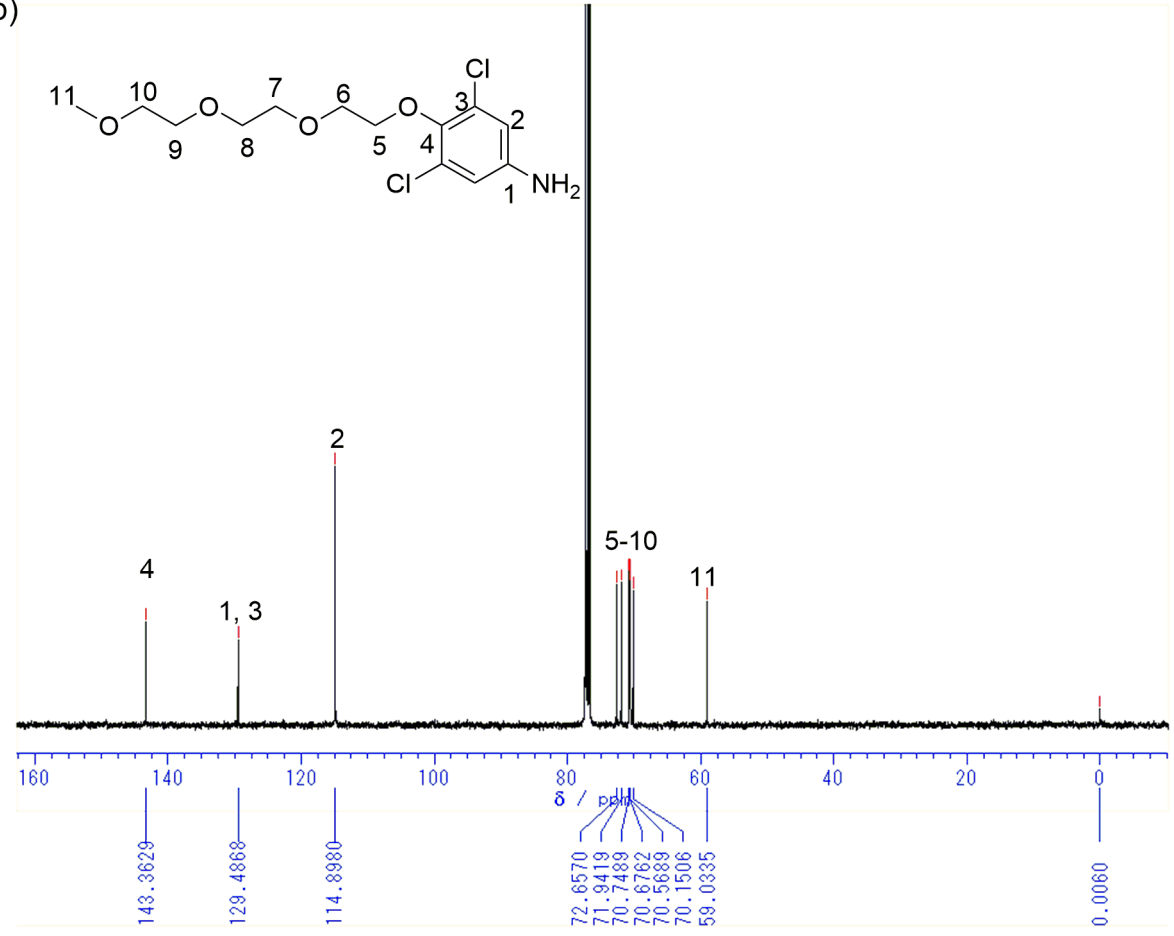

Figure S9. (a) ${ }^{1} \mathrm{H} \quad(400 \quad \mathrm{MHz})$ and $\quad$ (b) ${ }^{13} \mathrm{C} \quad \mathrm{NMR} \quad$ spectra $\quad\left(\begin{array}{lllll}100 & \mathrm{MHz}\end{array}\right)$ of 3,5-dichloro-4-(2-(2-(2-methoxyethoxy)ethoxy)ethoxy)aniline in $\mathrm{CDCl}_{3}$. 
<smiles>COCCOc1c(Cl)cc(N)cc1Cl</smiles>

(a)

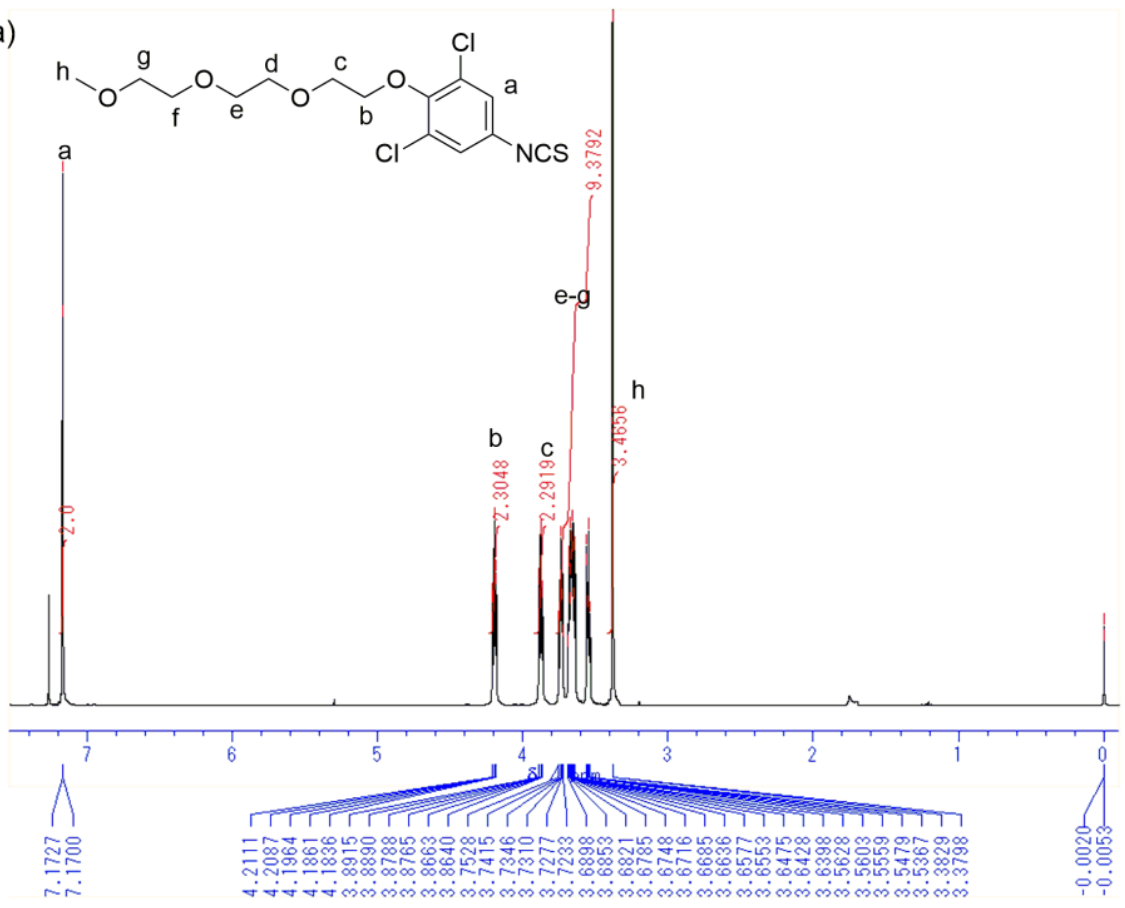

(b)
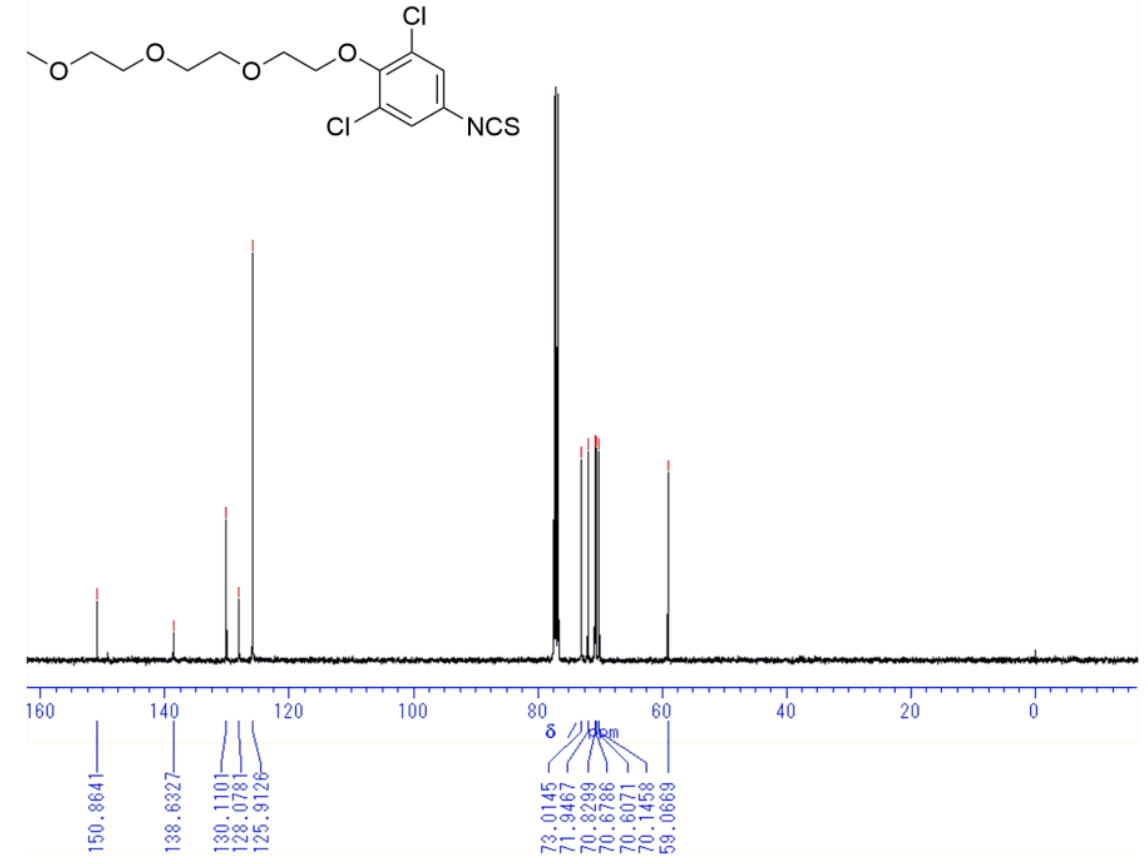

Figure S10. (a) ${ }^{1} \mathrm{H}(400 \mathrm{MHz})$ and (b) ${ }^{13} \mathrm{C}$ NMR spectra $(100 \mathrm{MHz})$ of

1,3-dichloro-5-isothiocyanato-2-(2-(2-(2-methoxyethoxy)ethoxy)ethoxy)benzene in $\mathrm{CDCl}_{3}$. 


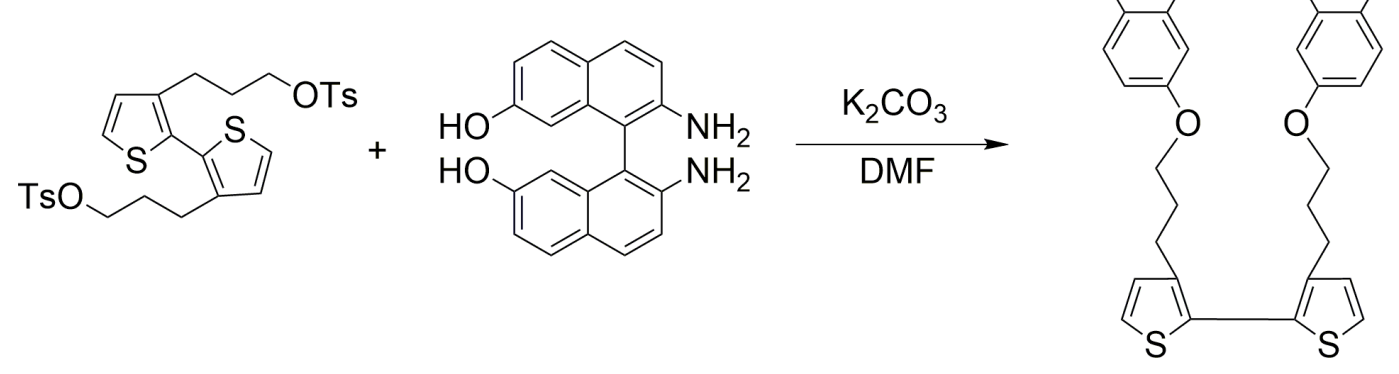

(a)

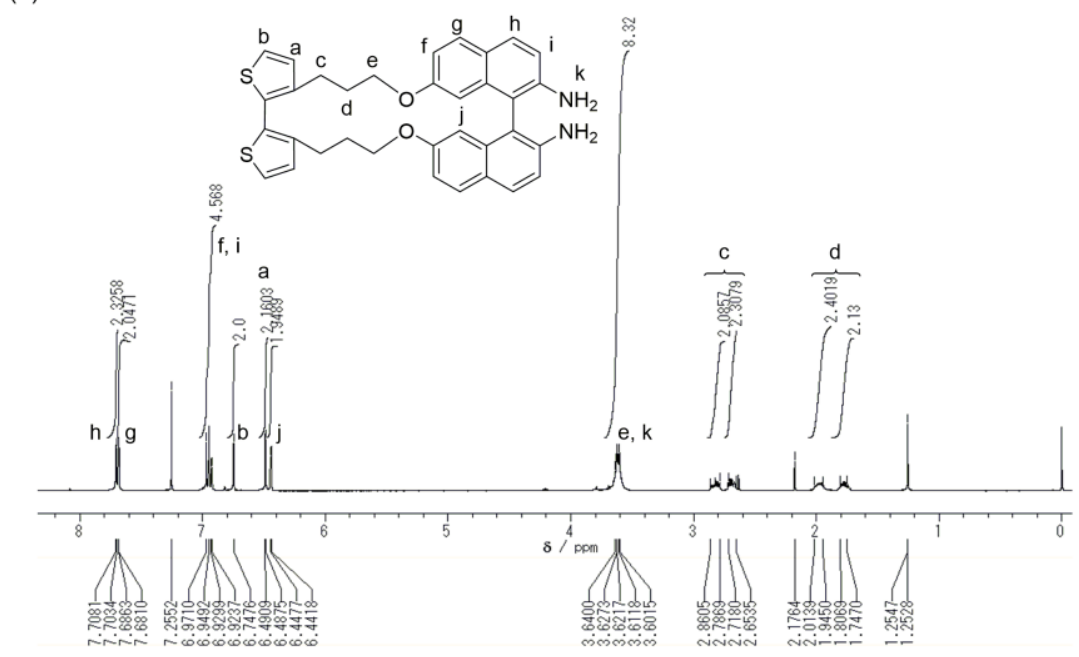

(b)

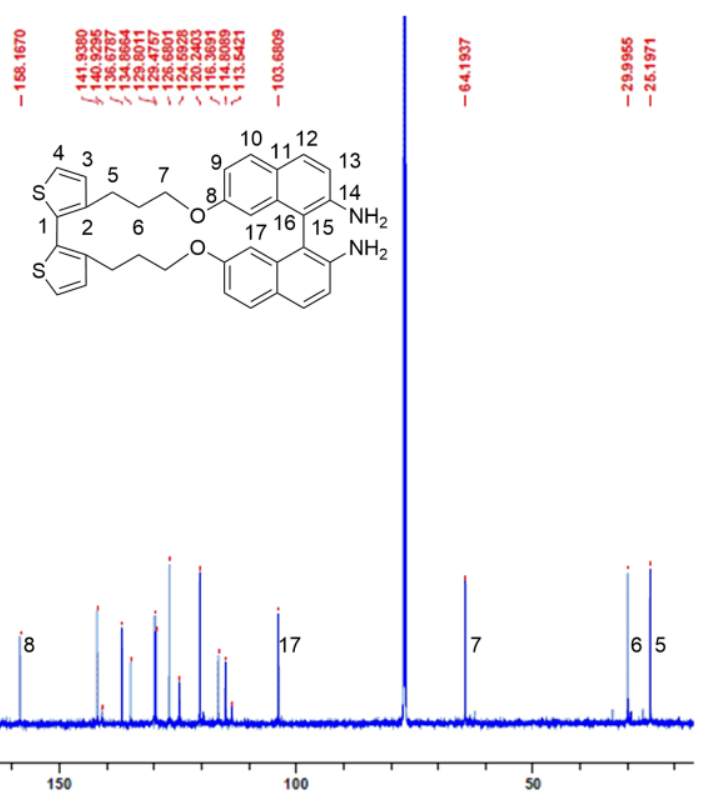

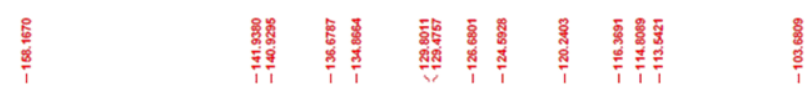

Figure S11. (a) ${ }^{1} \mathrm{H}(400 \mathrm{MHz})$ and (b) ${ }^{13} \mathrm{C}$ NMR spectra (100 MHz) of binaphthyl-bithiophene conjugate in $\mathrm{CDCl}_{3}$. 

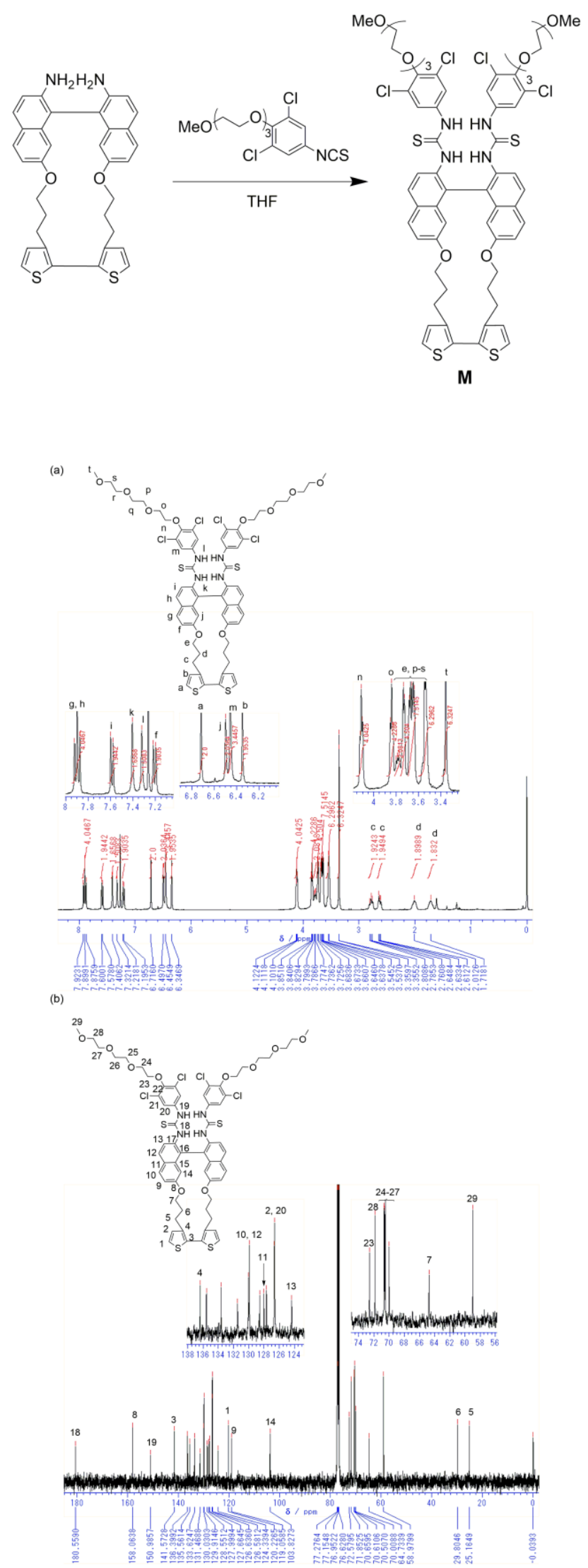

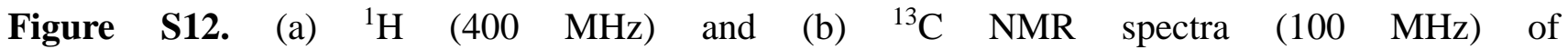
bisthiourea-binaphthyl-bithiophene conjugate $(\mathbf{M})$ in $\mathrm{CDCl}_{3}$. 
(a)

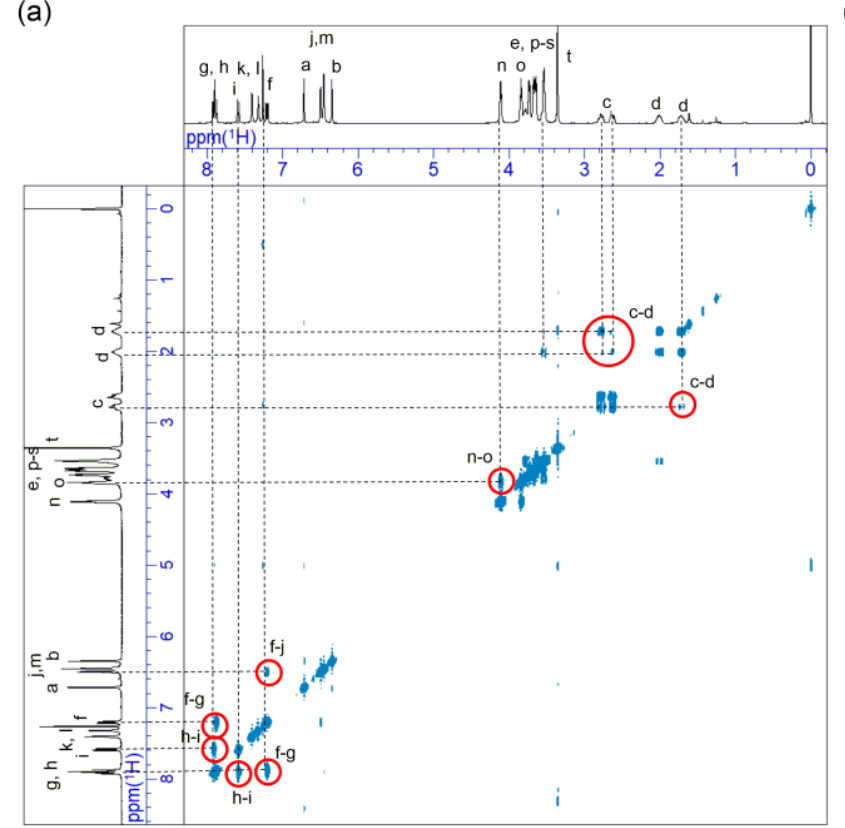

(b)

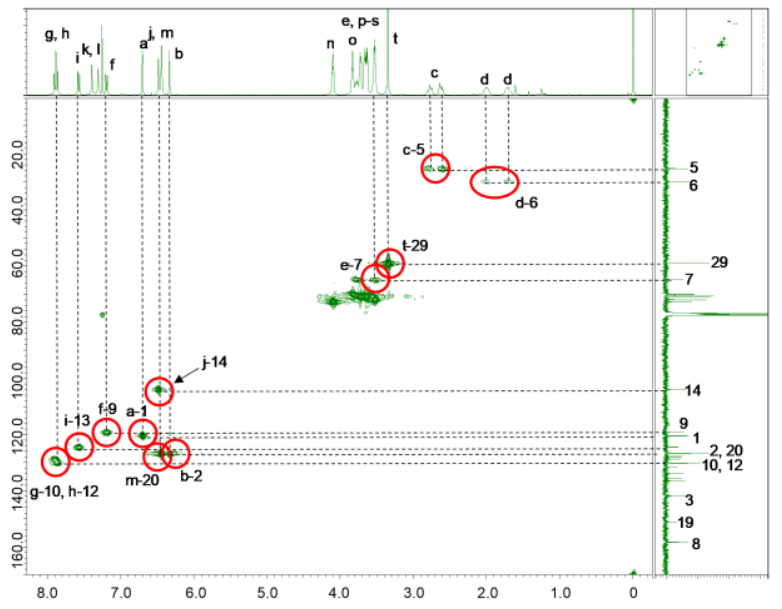

(c)

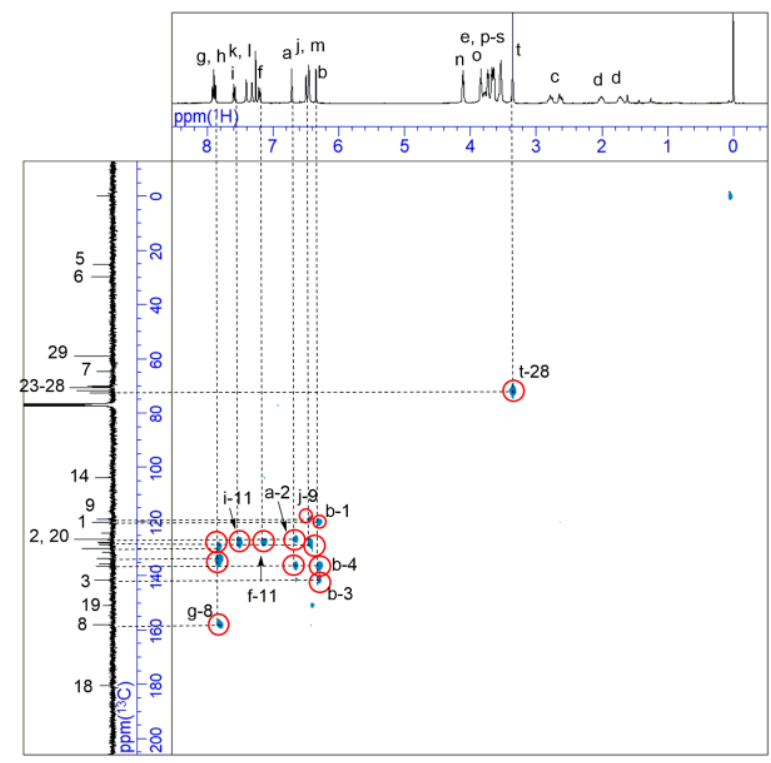

Figure S13. (a) COSY (400 MHz), (b) HMQC (100 MHz), and (c) HMBC spectra (100 MHz) of M in $\mathrm{CDCl}_{3}$.

(b) $13 \mathrm{C}\{1 \mathrm{H}\} \mathrm{NMR}\left(100 \mathrm{MHz}, \mathrm{CDCl}_{3}\right) \delta 130.0,129.9,128.0,127.7,124.3,120.2,119.1,103.8,64.7$, 59.0 .

(c) $13 \mathrm{C}\{1 \mathrm{H}\} \mathrm{NMR}\left(100 \mathrm{MHz}, \mathrm{CDCl}_{3}\right) \delta 158.1,141.6,136.4,133.6,130.0,129.9,128.0,126.6$, 120.2, 119.1 71.9. 


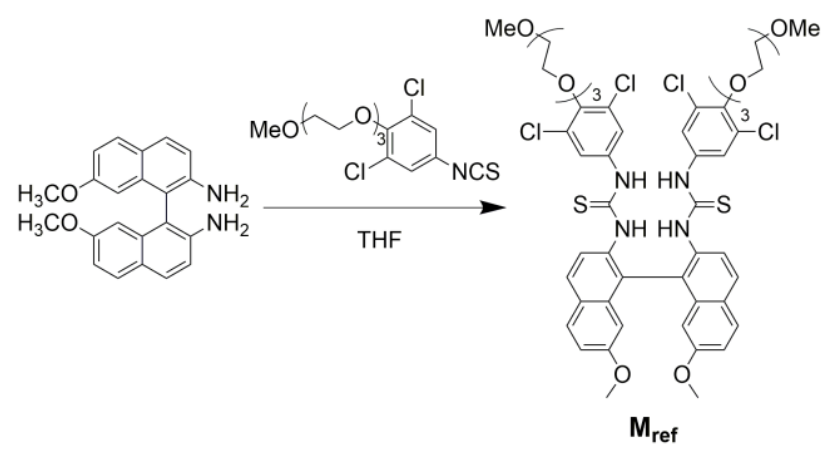

(a)

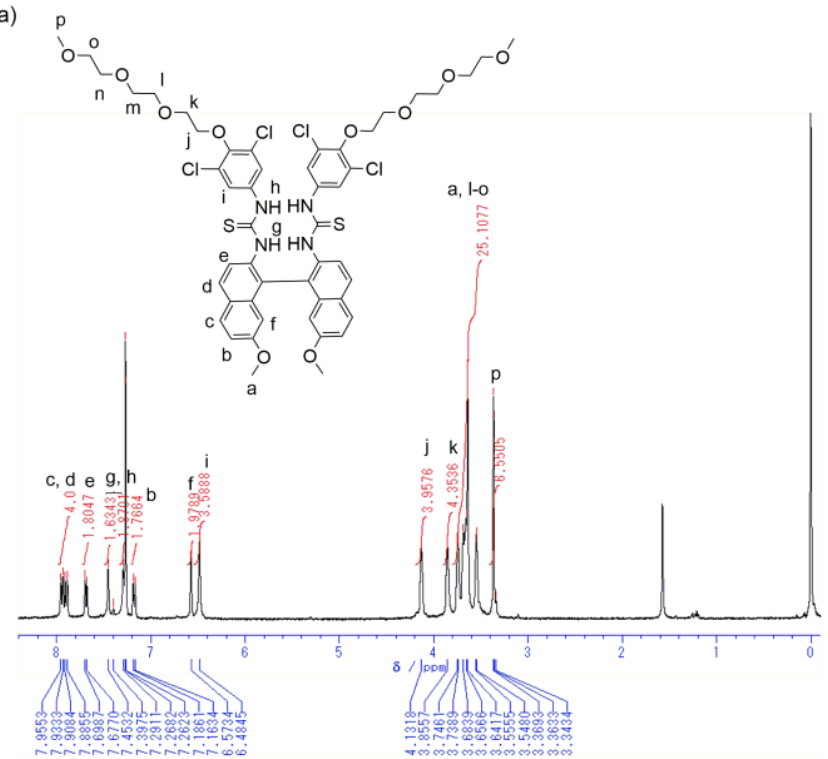

(b)

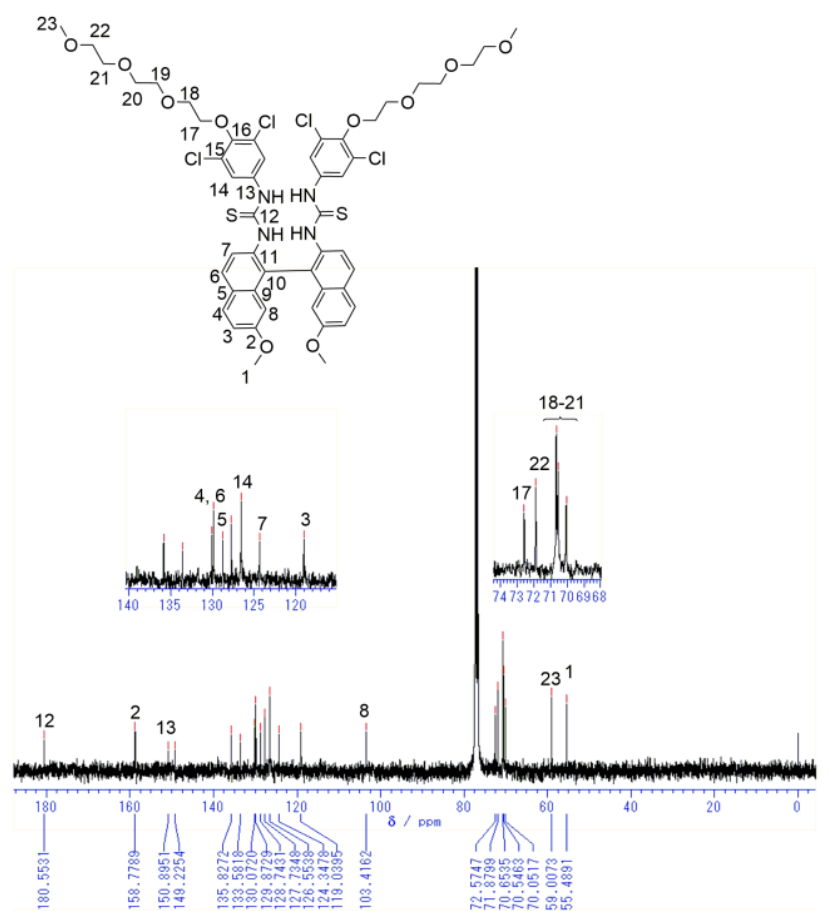

Figure S14. (a) ${ }^{1} \mathrm{H}(400 \mathrm{MHz})$ and (b) ${ }^{13} \mathrm{C}$ NMR spectra $(100 \mathrm{MHz})$ of $\mathbf{M}_{\text {ref }}$ in $\mathrm{CDCl}_{3}$. 

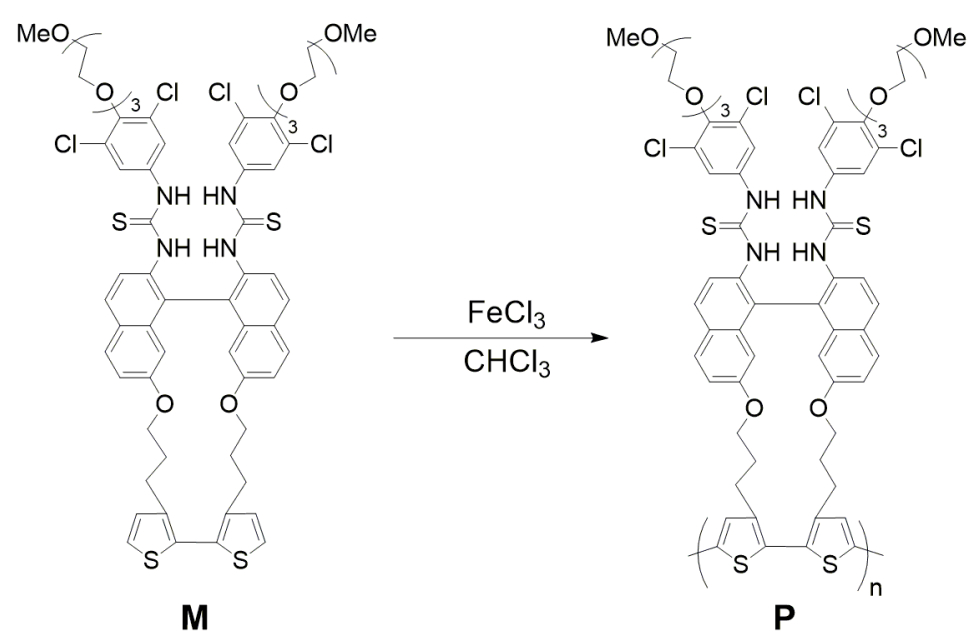

(a)

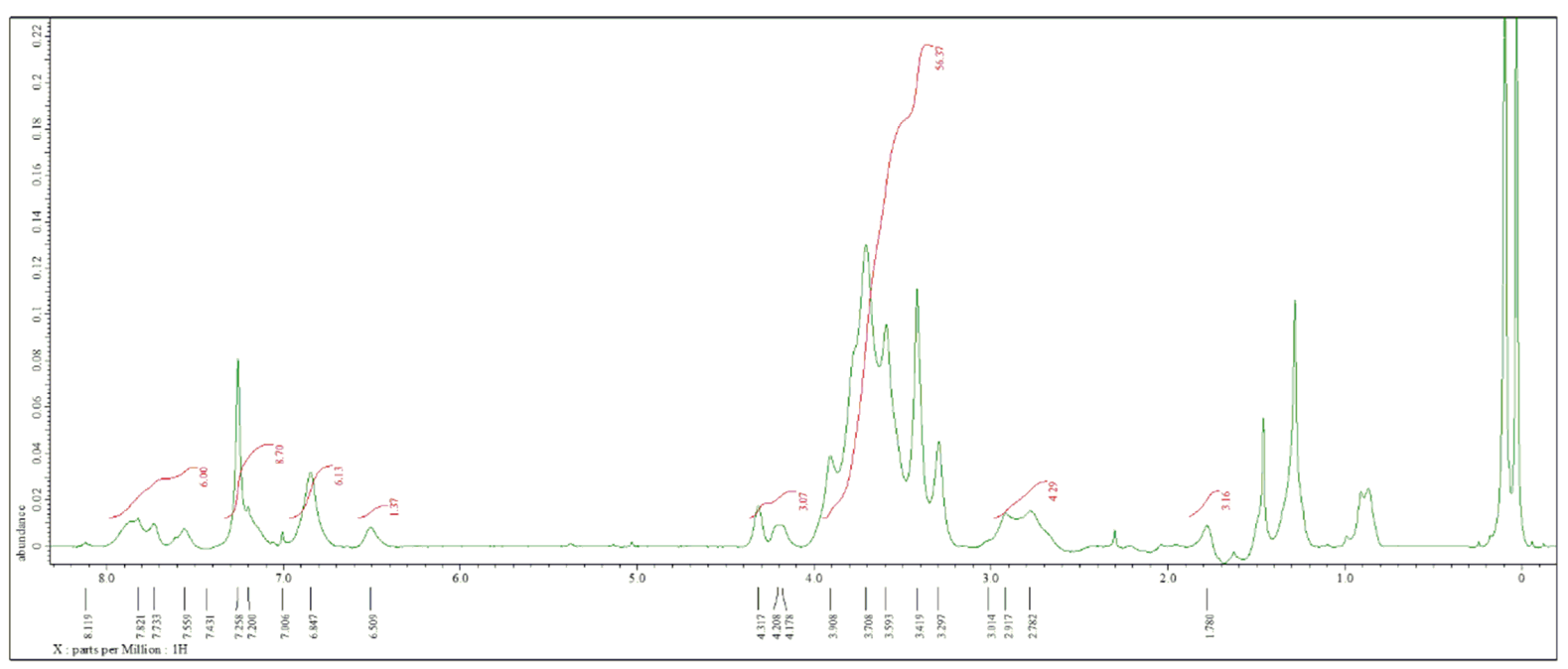

(b)

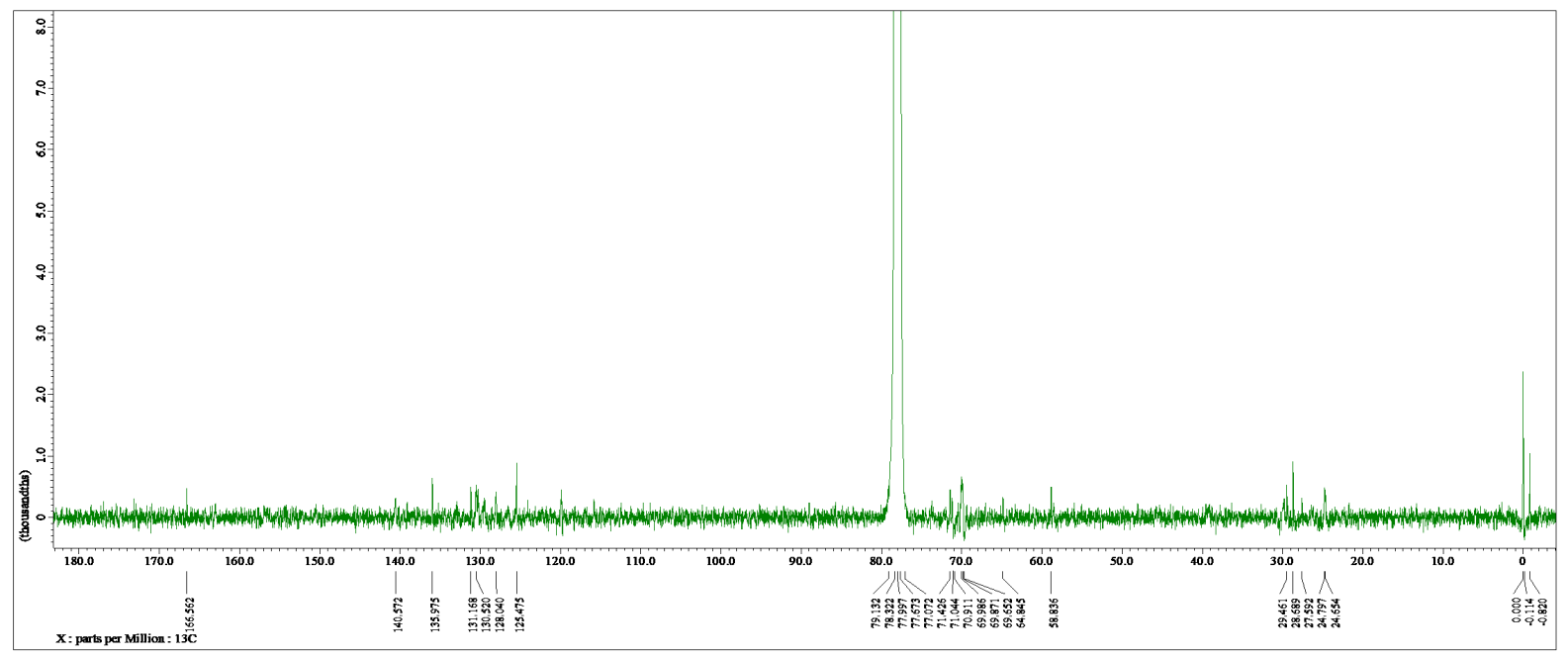

Figure S15. (a) ${ }^{1} \mathrm{H}(400 \mathrm{MHz})$ and (b) ${ }^{13} \mathrm{C}$ NMR spectra $(100 \mathrm{MHz})$ of $\mathbf{P}$ in $\mathrm{CDCl}_{3}$. 
(a)

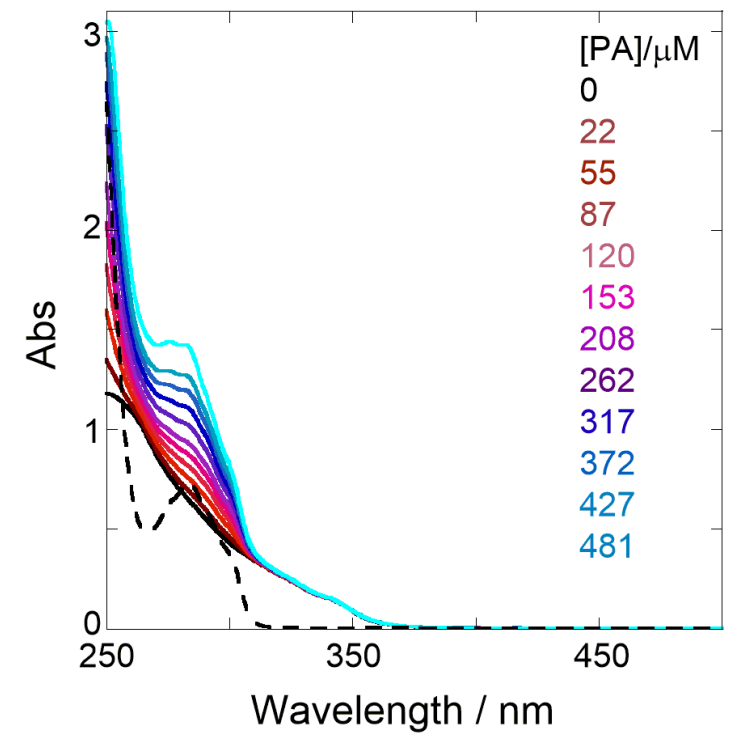

(b)

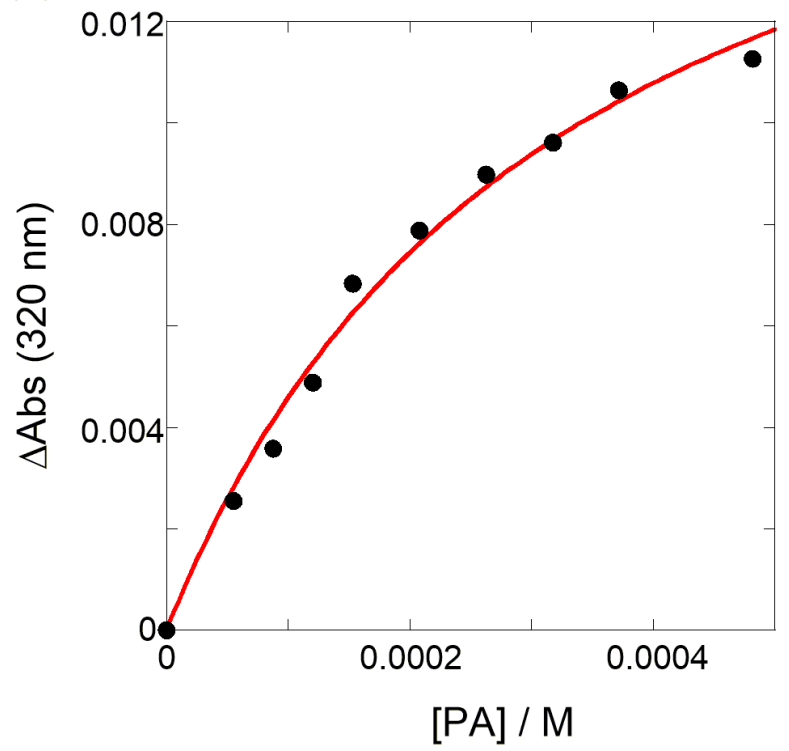

Figure S16. (a) UV/vis spectral changes of a chloroform solution ( $<2$ vol.\% DMF) of $\mathbf{M}(17.0 \mu \mathrm{M}$, black) upon gradual addition of PA $\left(22-481 \mu \mathrm{M}\right.$, colored lines) at $15{ }^{\circ} \mathrm{C}$; the dotted line shows the UV spectrum of PA only. (b) Non-linear least-squares fitting, assuming 1:1 stoichiometry, to determine the binding constant at $15^{\circ} \mathrm{C}$.

(a)

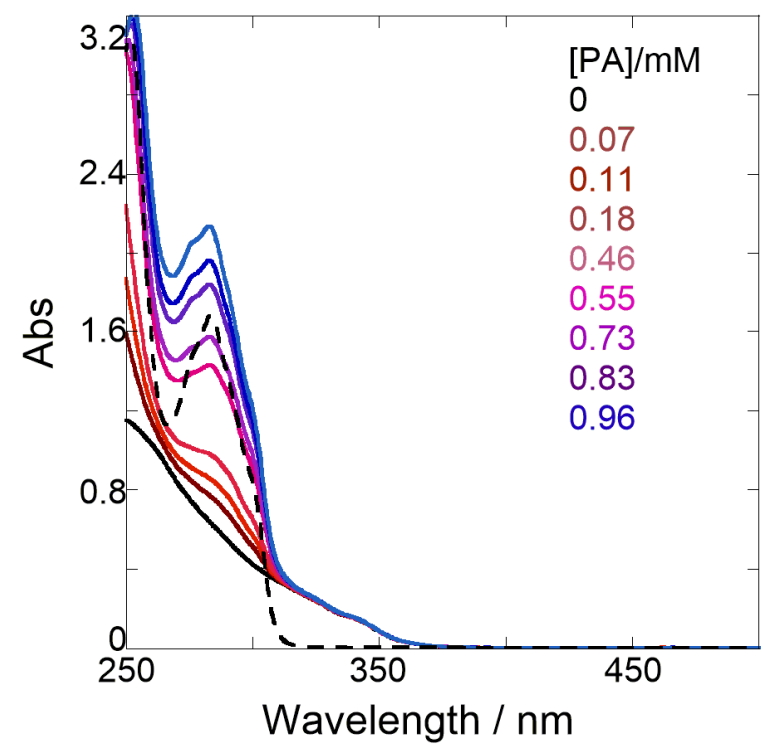

(b)

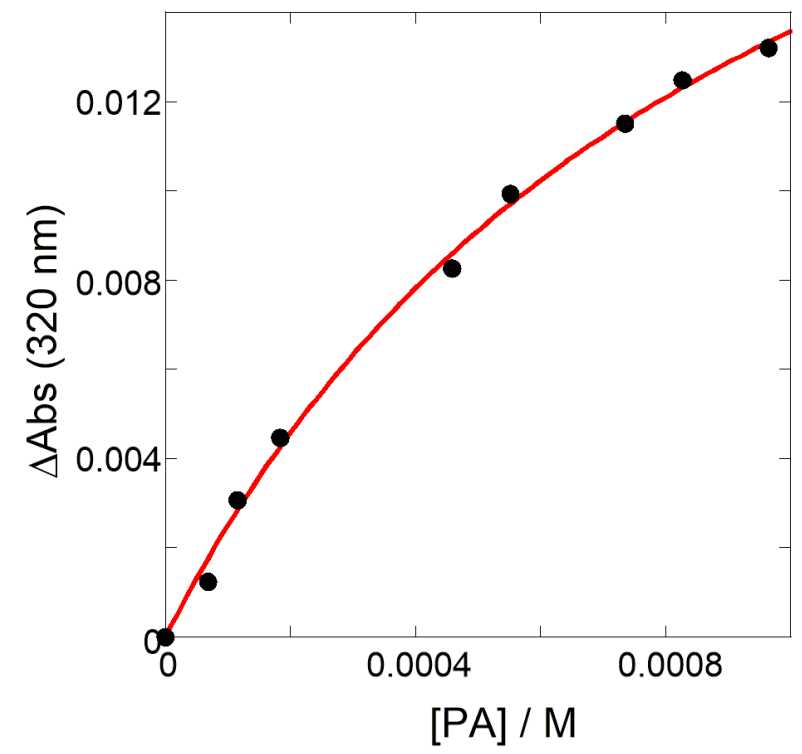

Figure S17. (a) UV/vis spectral changes of a chloroform solution ( $<2$ vol.\% DMF) of $\mathbf{M}(17.2 \mu \mathrm{M}$, black) upon gradual addition of PA $\left(0.07-0.96 \mathrm{mM}\right.$, colored lines) at $20^{\circ} \mathrm{C}$; the dotted line shows the UV spectrum of PA only. (b) Non-linear least-squares fitting, assuming 1:1 stoichiometry, to determine the binding constant at $20^{\circ} \mathrm{C}$. 
(a)

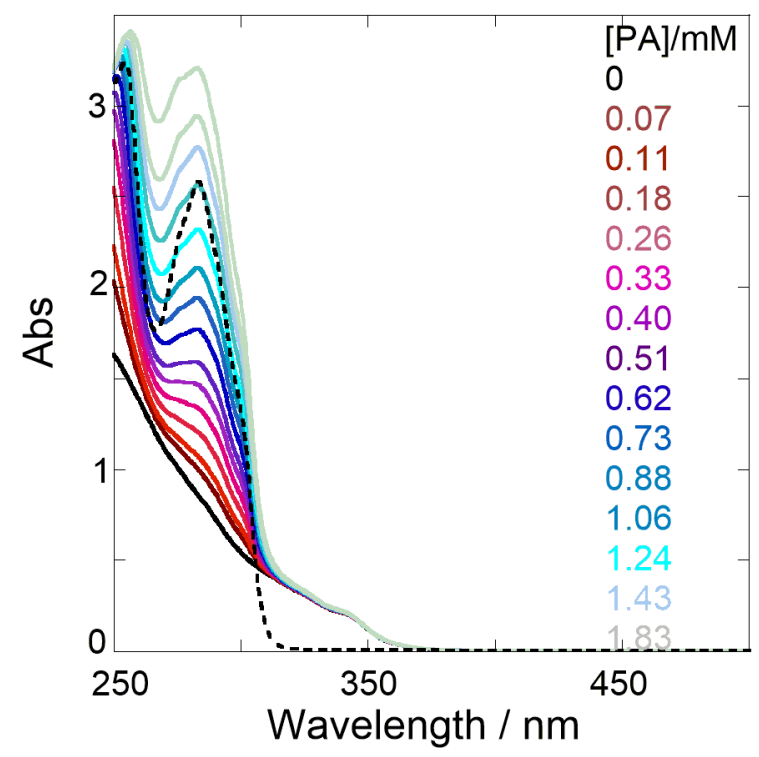

(b)

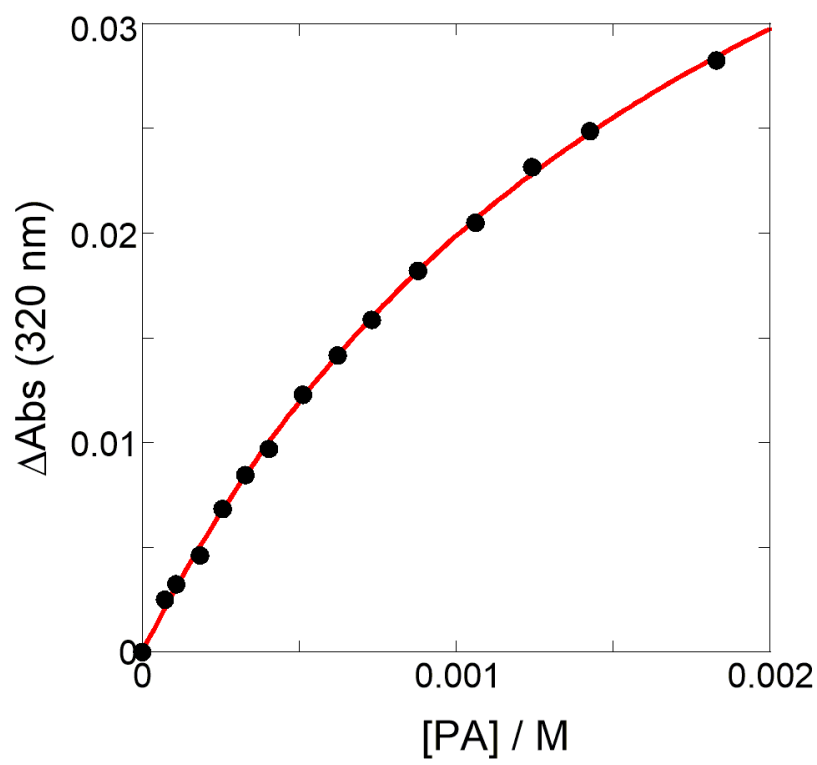

Figure S18. (a) UV/vis spectral changes of a chloroform solution ( $<2$ vol.\% DMF) of M ( $21.9 \mu \mathrm{M}$, black) upon gradual addition of PA $\left(0.07-1.83 \mathrm{mM}\right.$, colored lines) at $25^{\circ} \mathrm{C}$; the dotted line shows the UV spectrum of PA only. (b) Non-linear least-squares fitting, assuming 1:1 stoichiometry, to determine the binding constant at $25^{\circ} \mathrm{C}$.

(a)

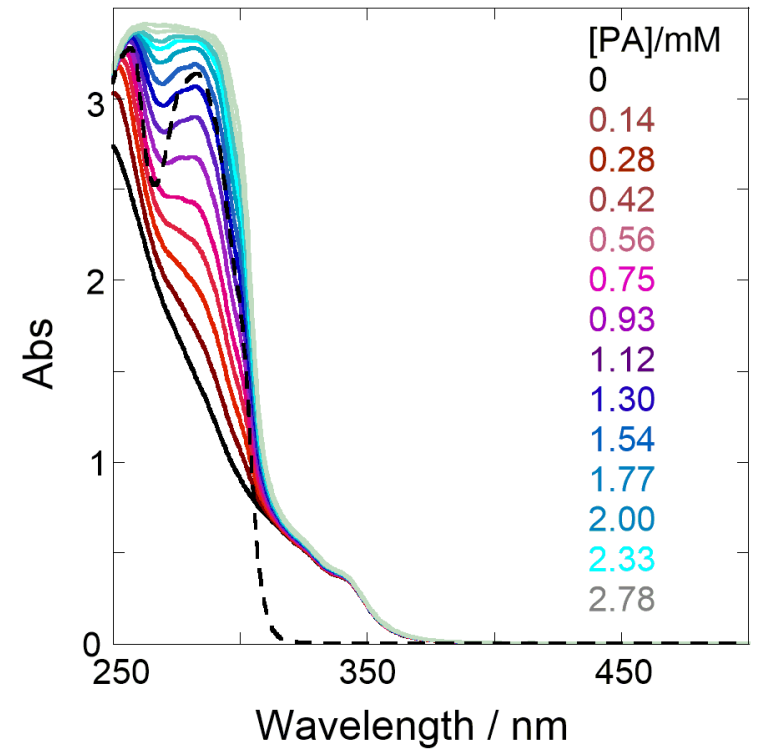

(b)

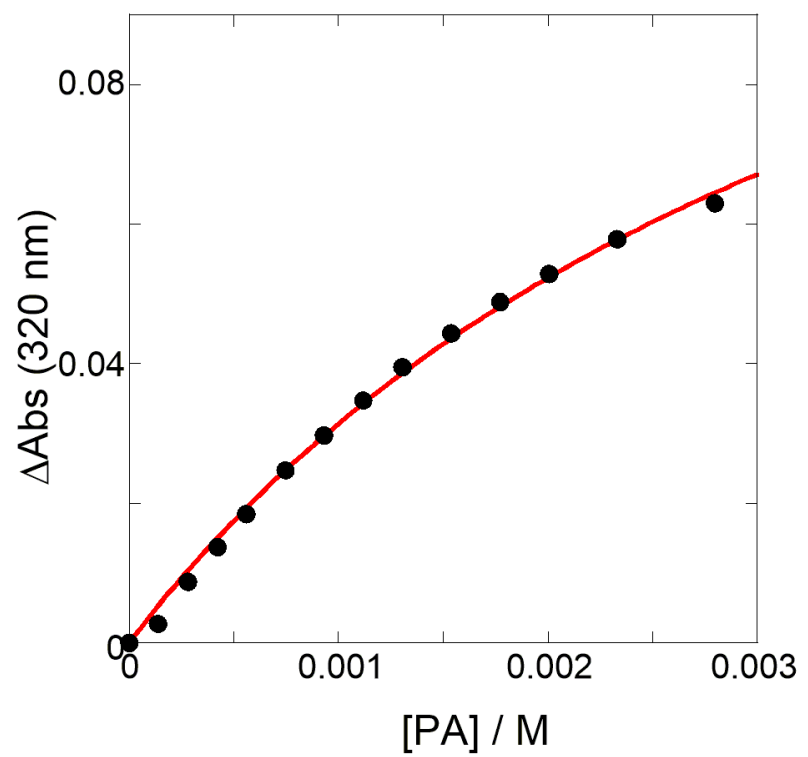

Figure S19. (a) UV/vis spectral changes of a chloroform solution ( $<2$ vol.\% DMF) of M (36.5 $\mu \mathrm{M}$, black) upon gradual addition of PA $\left(0.14-2.78 \mathrm{mM}\right.$, colored lines) at $35^{\circ} \mathrm{C}$; the dotted line shows the UV spectrum of PA only. (b) Non-linear least-squares fitting, assuming 1:1 stoichiometry, to determine the binding constant at $35^{\circ} \mathrm{C}$. 
(a)

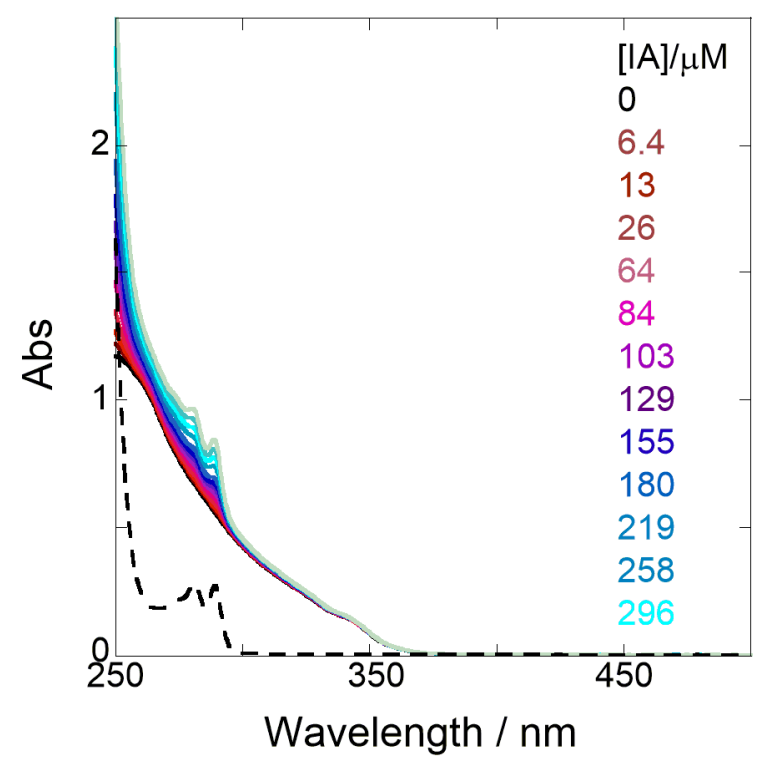

(b)

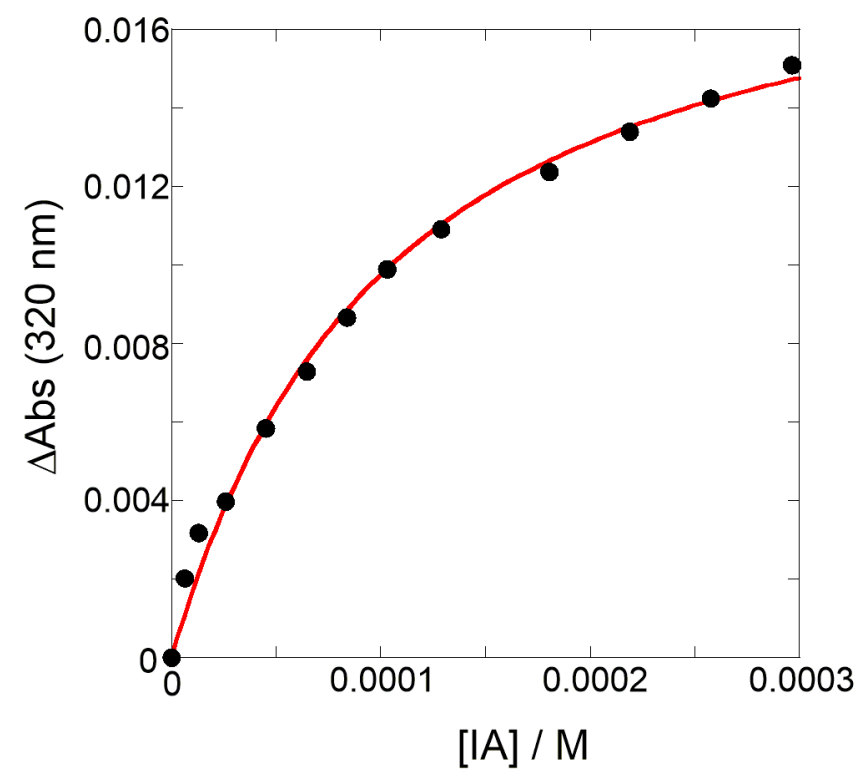

Figure S20. (a) UV/vis spectral changes of a chloroform solution ( $<2$ vol.\% DMF) of M (17.0 $\mu \mathrm{M}$, black) upon gradual addition of IA $\left(6.4-296 \mu \mathrm{M}\right.$, colored lines) at $15{ }^{\circ} \mathrm{C}$; the dotted line shows the UV spectrum of IA only. (b) Non-linear least-squares fitting, assuming 1:1 stoichiometry, to determine the binding constant at $15^{\circ} \mathrm{C}$.

(a)

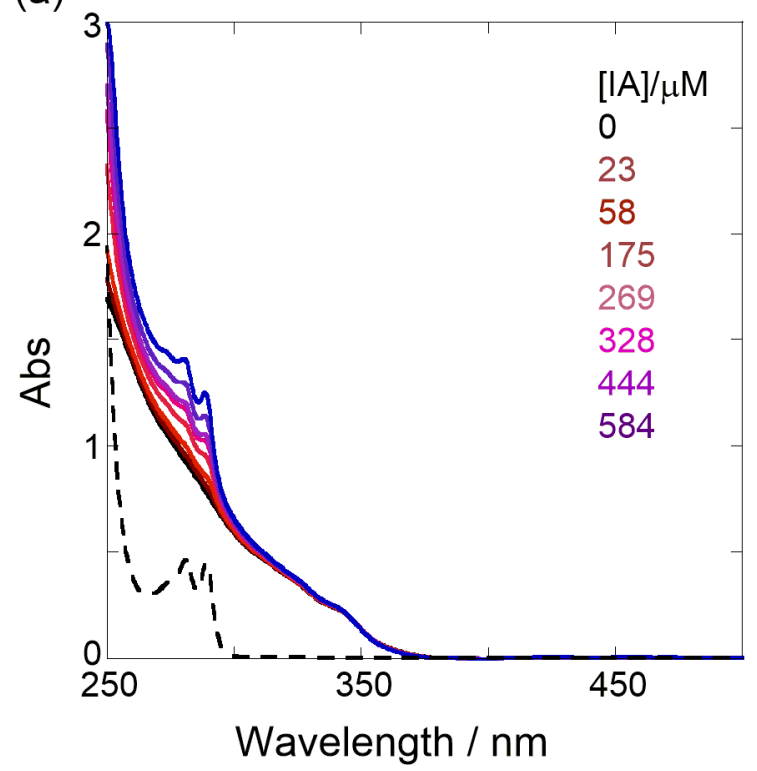

(b)

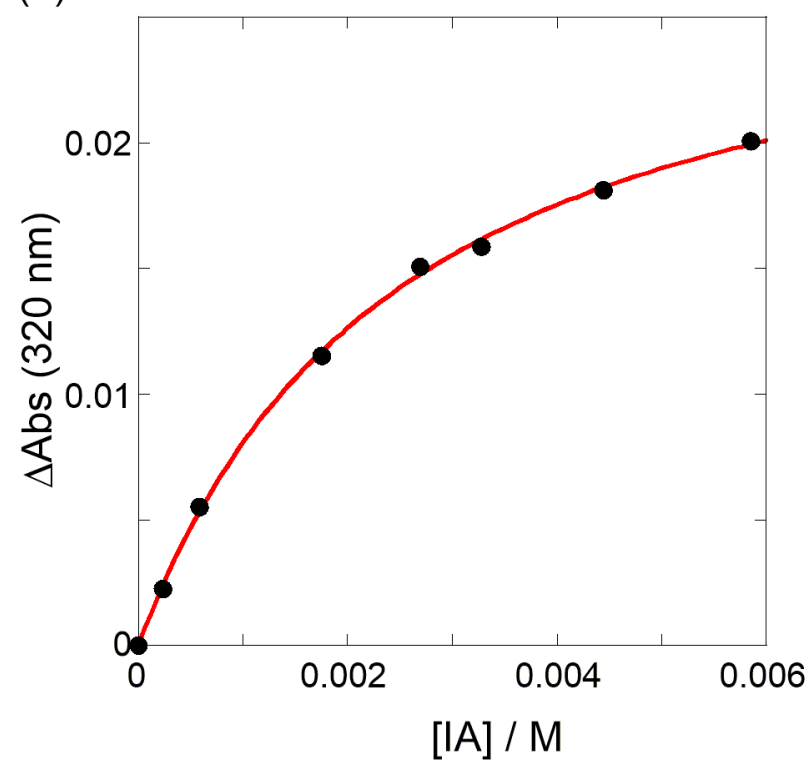

Figure S21. (a) UV/vis spectral changes of a chloroform solution ( $<2$ vol.\% DMF) of $\mathbf{M}(25.1 \mu \mathrm{M}$, black) upon gradual addition of IA (23-584 $\mu \mathrm{M}$, colored lines) at $25{ }^{\circ} \mathrm{C}$; the dotted line shows the UV spectrum of IA only. (b) Non-linear least-squares fitting, assuming 1:1 stoichiometry, to determine the binding constant at $25^{\circ} \mathrm{C}$. 
(a)

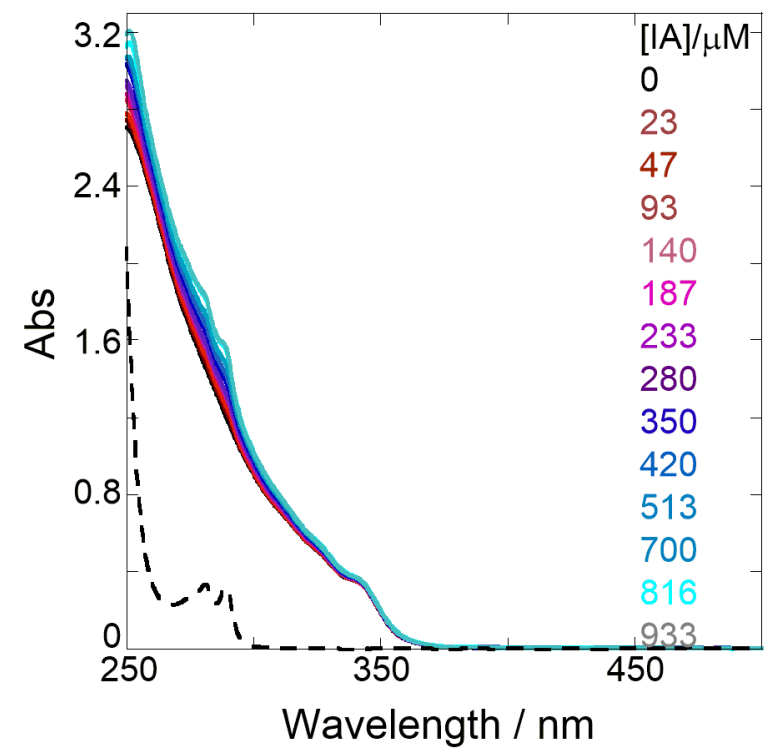

(b)

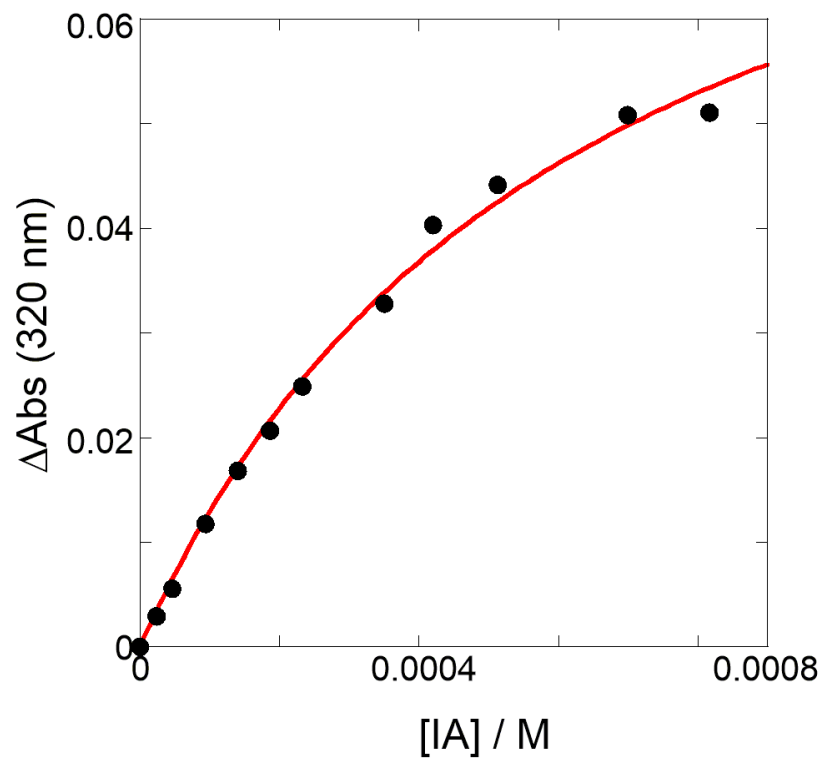

Figure S22. (a) UV/vis spectral changes of a chloroform solution ( $<2$ vol.\% DMF) of M (36.1 $\mu \mathrm{M}$, black) upon gradual addition of IA $\left(23-933 \mu \mathrm{M}\right.$, colored lines) at $30{ }^{\circ} \mathrm{C}$; the dotted line shows the UV spectrum of IA only. (b) Non-linear least-squares fitting, assuming 1:1 stoichiometry, to determine the binding constant at $30^{\circ} \mathrm{C}$.

(a)

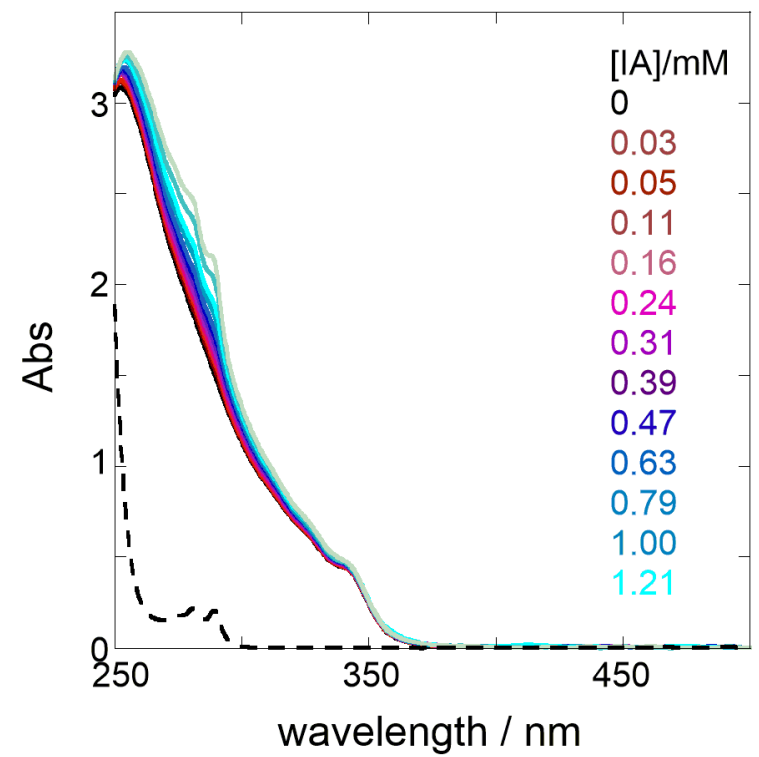

(b)

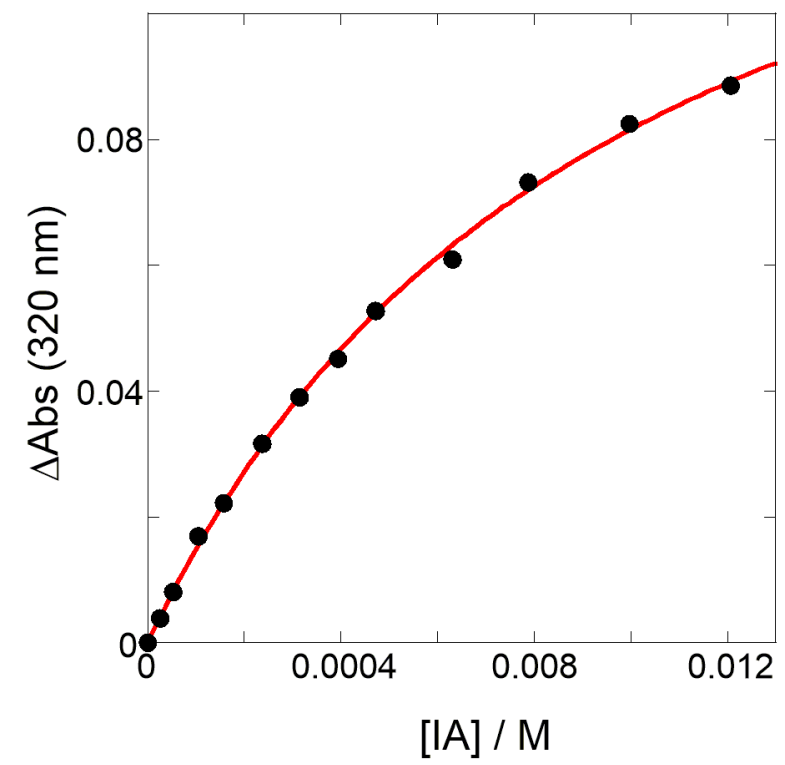

Figure S23. (a) UV/vis spectral changes of a chloroform solution (<2 vol.\% DMF) of $\mathbf{M}(45.7 \mu \mathrm{M}$, black) upon gradual addition of IA $\left(0.03-1.21 \mathrm{mM}\right.$, colored lines) at $35{ }^{\circ} \mathrm{C}$; the dotted line shows the UV spectrum of IA only. (b) Non-linear least-squares fitting, assuming 1:1 stoichiometry, to determine the binding constant at $35^{\circ} \mathrm{C}$. 
(a)

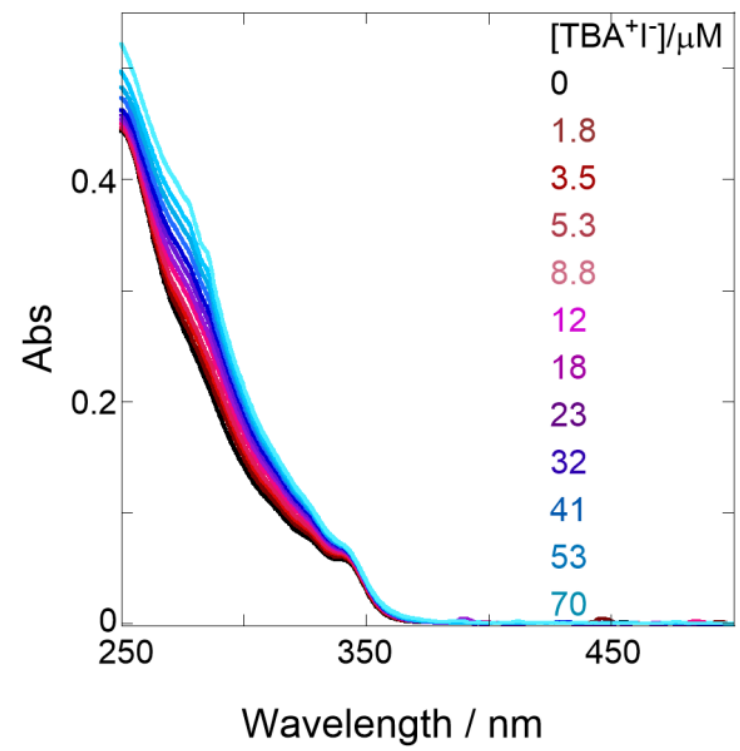

(b)

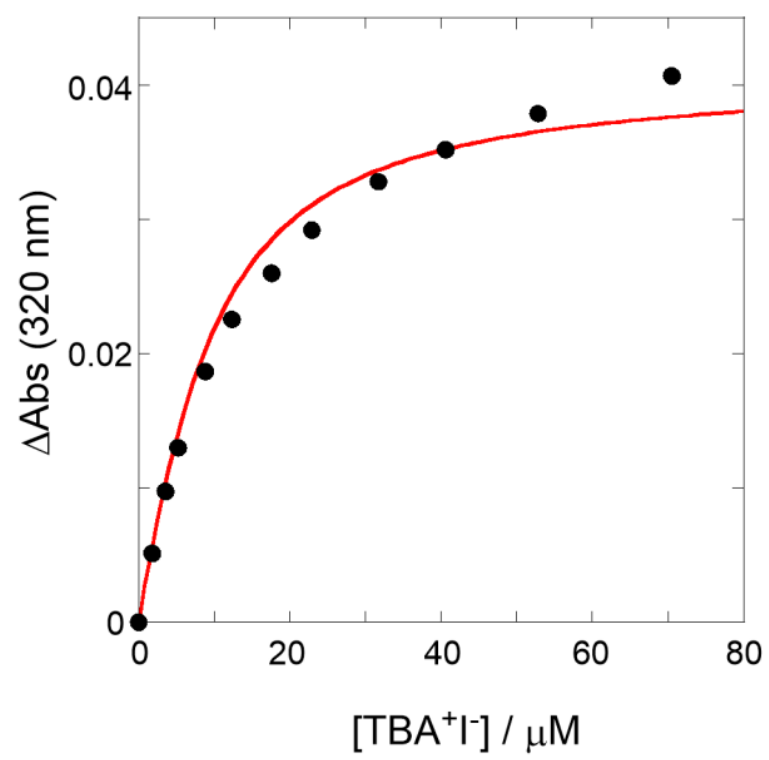

Figure S24. (a) UV/vis spectral changes of a chloroform solution of $\mathbf{M}(6.53 \mu \mathrm{M}$, black) upon gradual addition of $\mathrm{TBA}^{+} \mathrm{I}^{-}\left(1.8-70 \mu \mathrm{M}\right.$, colored lines) at $20^{\circ} \mathrm{C}$. (b) Non-linear least-squares fitting, assuming $1: 1$ stoichiometry, to determine the binding constant at $20^{\circ} \mathrm{C}$.

(a)

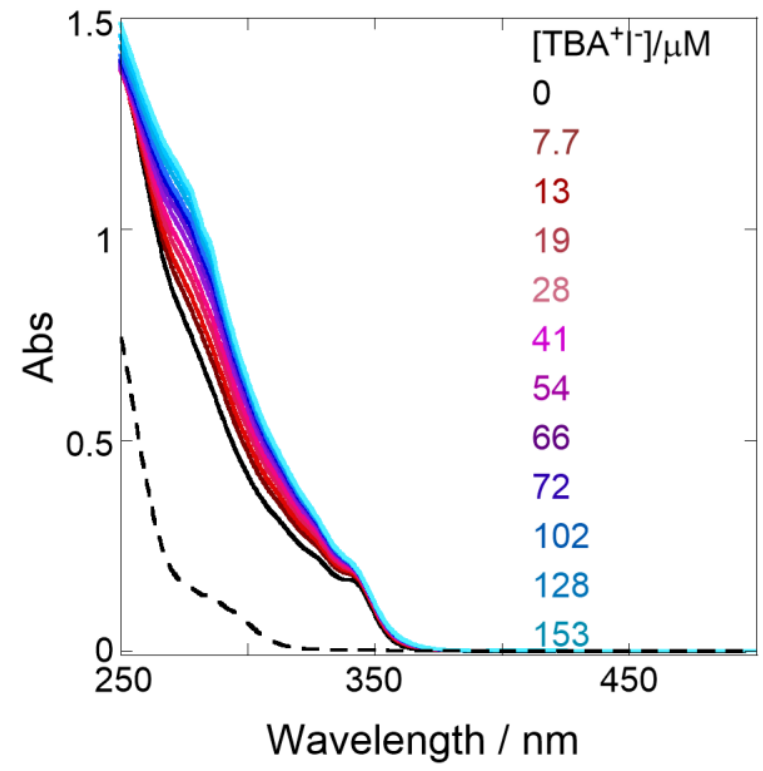

(b)

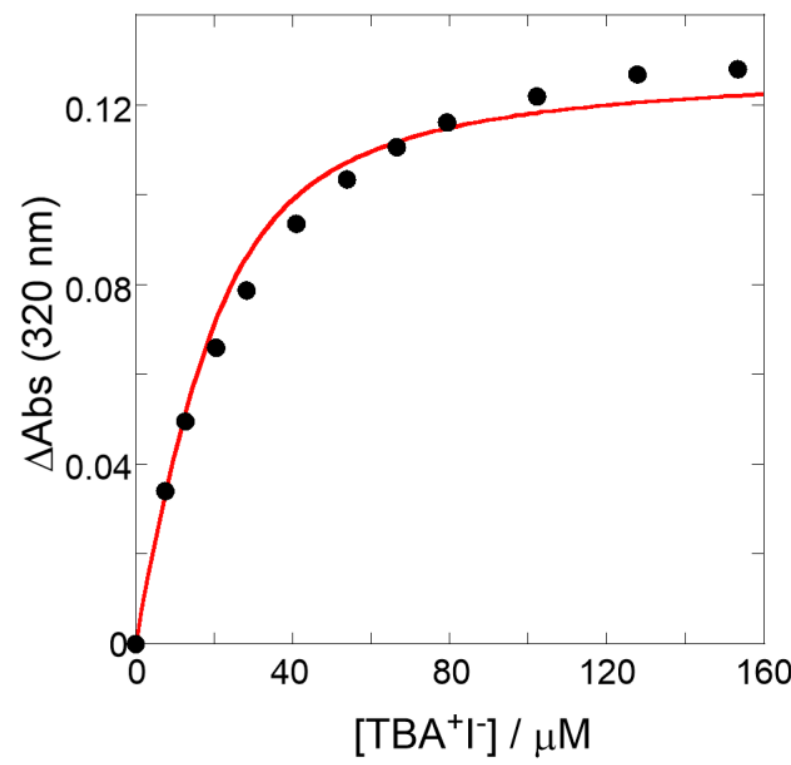

Figure S25. (a) UV/vis spectral changes of a chloroform solution of $\mathbf{M}(19.3 \mu \mathrm{M}$, black) upon gradual addition of $\mathrm{TBA}^{+} \mathrm{I}^{-}\left(7.7-153 \mu \mathrm{M}\right.$, colored lines) at $30{ }^{\circ} \mathrm{C}$; the dotted line shows the UV spectrum of $\mathrm{TBA}^{+} \mathrm{I}^{-}$only. (b) Non-linear least-squares fitting, assuming 1:1 stoichiometry, to determine the binding constant at $30^{\circ} \mathrm{C}$. 
(a)

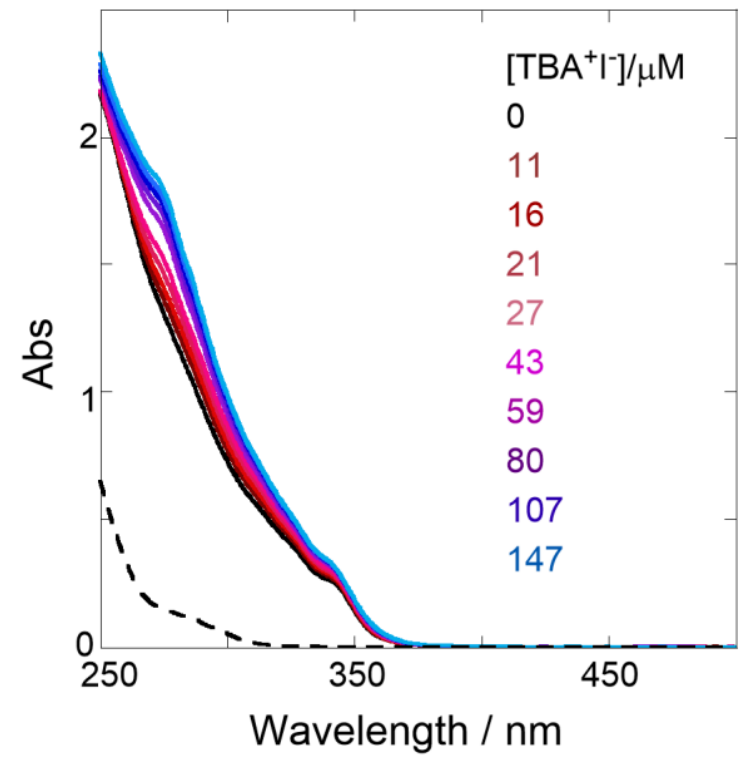

(b)

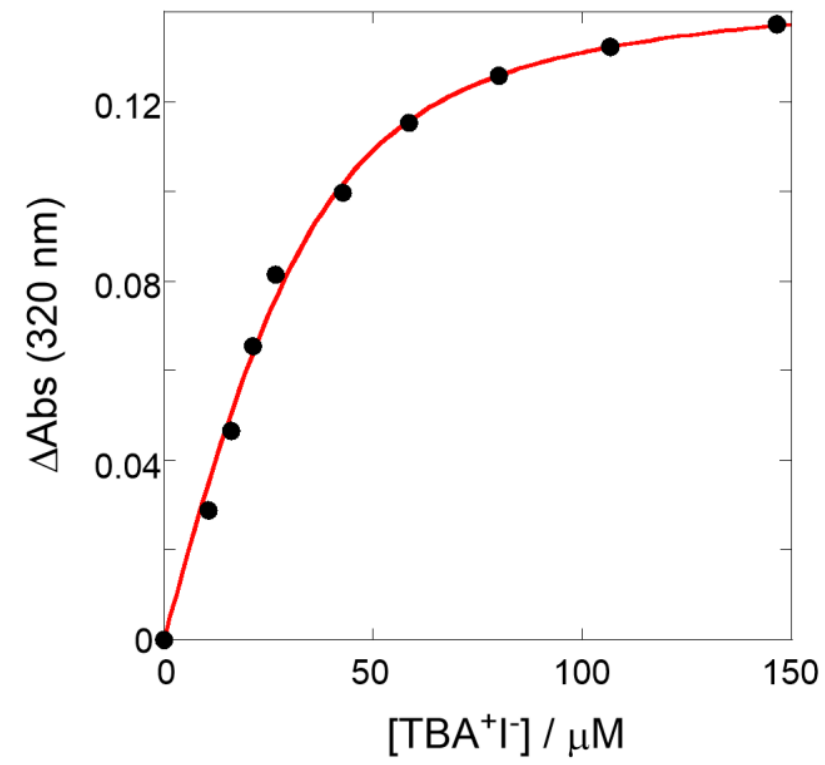

Figure S26. (a) UV/vis spectral changes of a chloroform solution of $\mathbf{M}(33.7 \mu \mathrm{M}$, black) upon gradual addition of $\mathrm{TBA}^{+} \mathrm{I}^{-}\left(11-147 \mu \mathrm{M}\right.$, colored lines) at $35{ }^{\circ} \mathrm{C}$; the dotted line shows the UV spectrum of $\mathrm{TBA}^{+} \mathrm{I}^{-}$only. (b) Non-linear least-squares fitting, assuming 1:1 stoichiometry, to determine the binding constant at $35^{\circ} \mathrm{C}$.

(a)

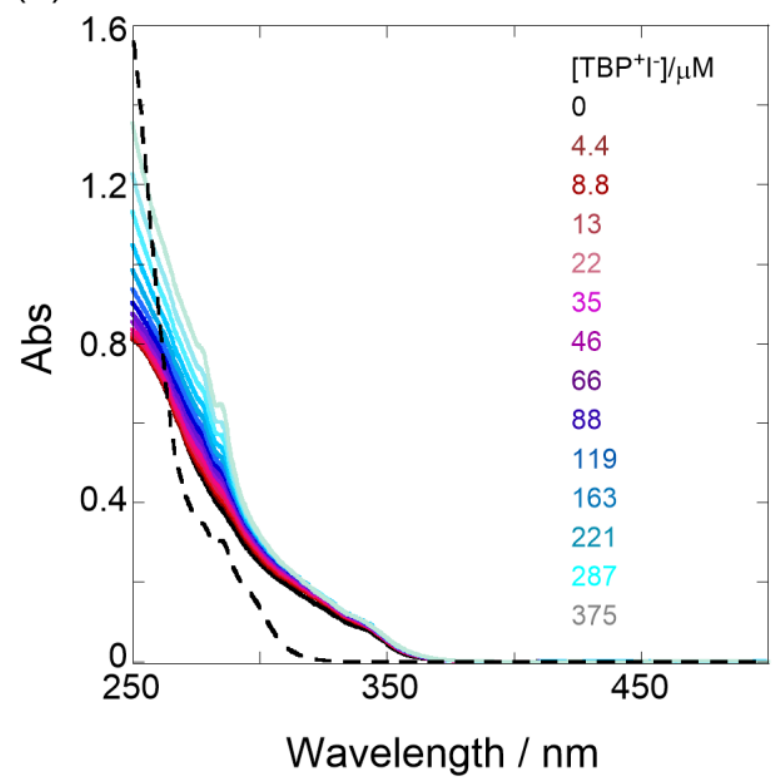

(b)

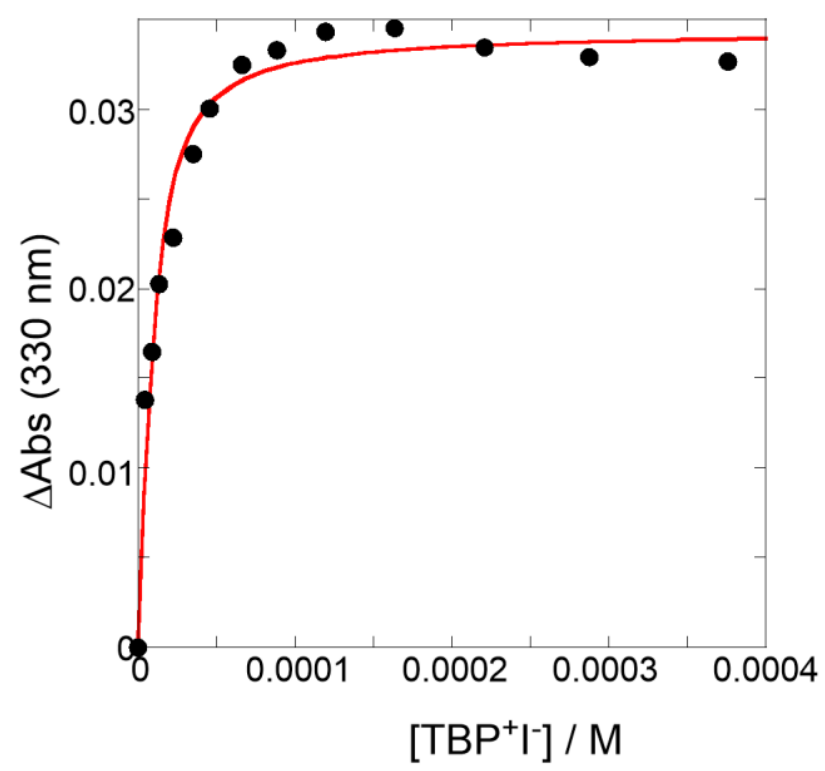

Figure S27. (a) UV/vis spectral changes of a chloroform solution of $\mathbf{M}(9.70 \mu \mathbf{M}$, black) upon gradual addition of $\mathrm{TBP}^{+} \mathrm{I}^{-}\left(4.4-375 \mu \mathrm{M}\right.$, colored lines) at $25{ }^{\circ} \mathrm{C}$; the dotted line shows the UV spectrum of $\mathrm{TBP}^{+} \mathrm{I}^{-}$only. (b) Non-linear least-squares fitting, assuming 1:1 stoichiometry, to determine the binding constant at $25^{\circ} \mathrm{C}$. 
(a)

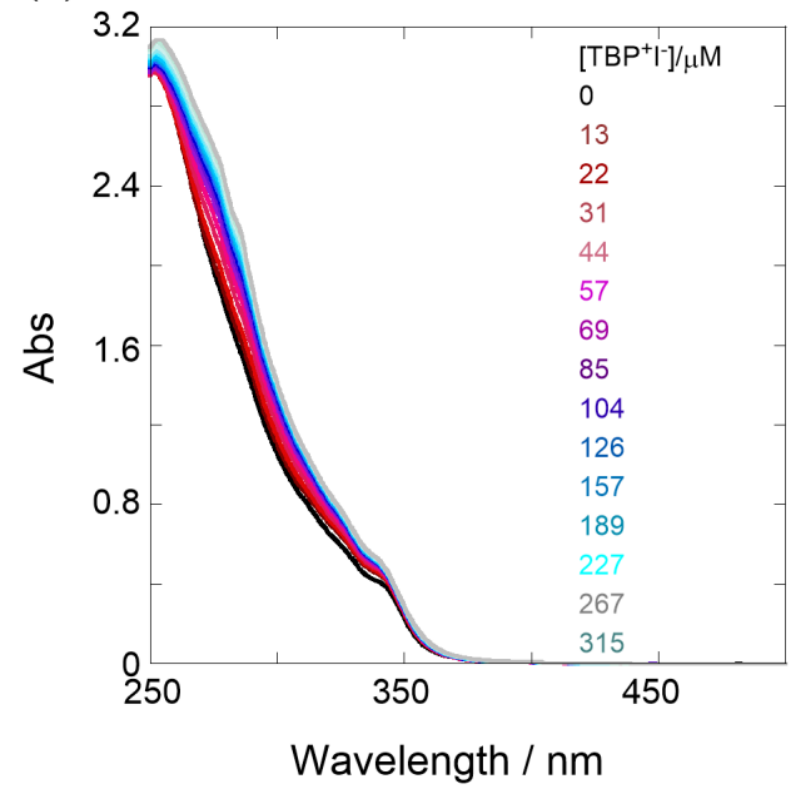

(b)

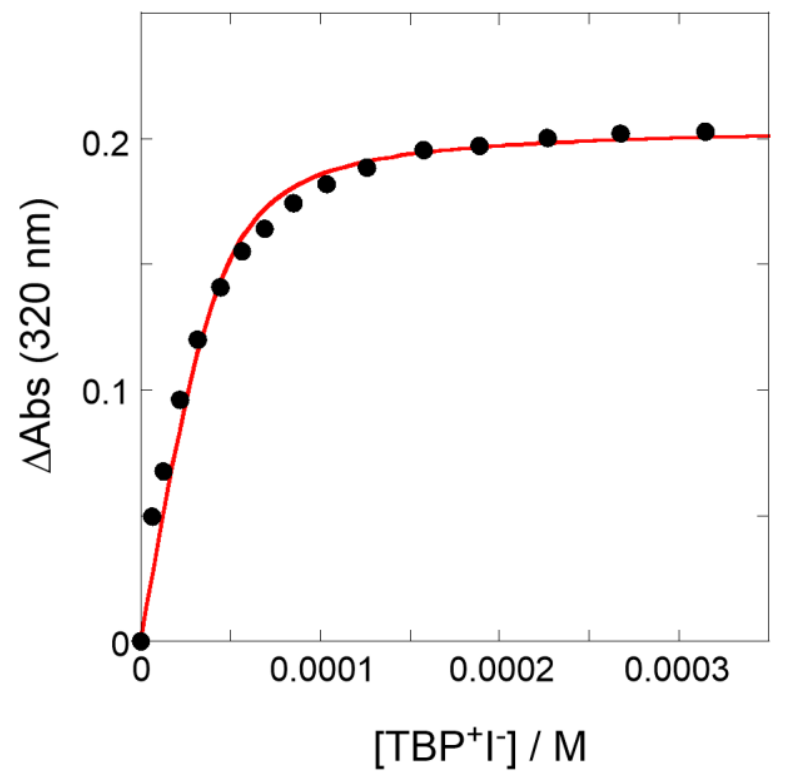

Figure S28. (a) UV/vis spectral changes of a chloroform solution of $\mathbf{M}(42.1 \mu \mathbf{M}$, black) upon gradual addition of $\mathrm{TBP}^{+} \mathrm{I}^{-}\left(13-315 \mu \mathrm{M}\right.$, colored lines) at $35^{\circ} \mathrm{C}$. (b) Non-linear least-squares fitting, assuming 1:1 stoichiometry, to determine the binding constant at $35^{\circ} \mathrm{C}$.

(a)

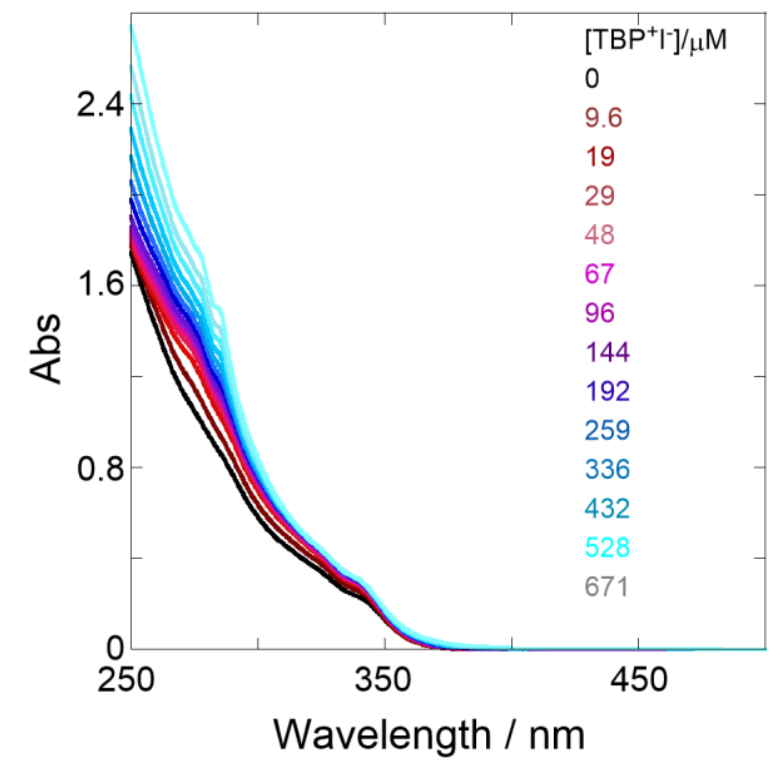

(b)

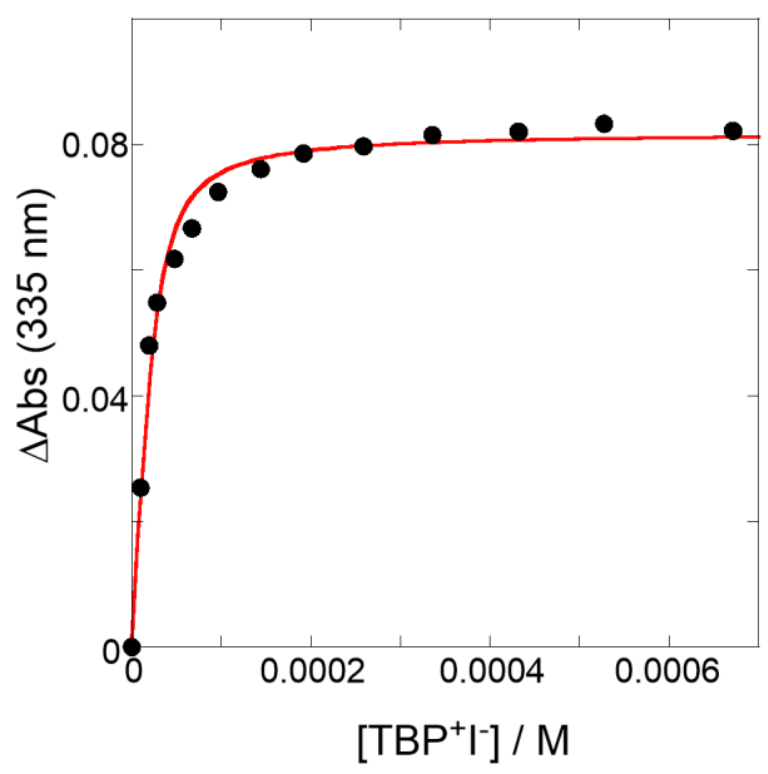

Figure S29. (a) UV/vis spectral changes of a chloroform solution of $\mathbf{M}(25.1 \mu \mathrm{M}$, black) upon gradual addition of $\mathrm{TBP}^{+} \mathrm{I}^{-}\left(9.6-671 \mu \mathrm{M}\right.$, colored lines) at $40{ }^{\circ} \mathrm{C}$. (b) Non-linear least-squares fitting, assuming 1:1 stoichiometry, to determine the binding constant at $40{ }^{\circ} \mathrm{C}$. 
(a)

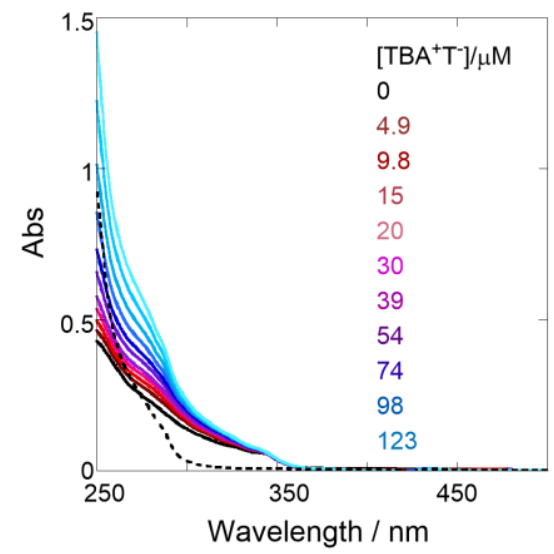

(b)

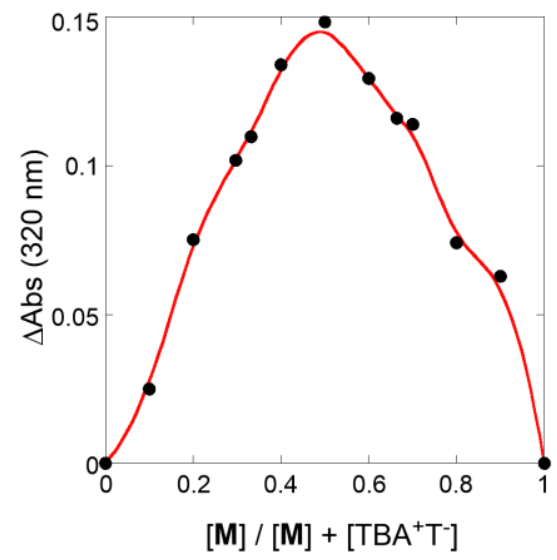

Figure S30. (a) UV/vis spectral changes of a chloroform solution of $\mathbf{M}(5.25 \mu \mathrm{M}$, black) upon gradual addition of $\mathrm{TBA}^{+} \mathrm{T}^{-}\left(4.9-123 \mu \mathrm{M}\right.$, colored lines) at $25^{\circ} \mathrm{C}$; the dotted line shows the UV spectrum of $\mathrm{TBP}^{+} \mathrm{T}^{-}$only. (b) Job plot of the absorbance changes at $320 \mathrm{~nm}$ upon complexation of $\mathrm{TBA}^{+} \mathrm{T}^{-}$with $\mathrm{M}$ in $\mathrm{CHCl}_{3}$ at $25^{\circ} \mathrm{C}$.

(a)

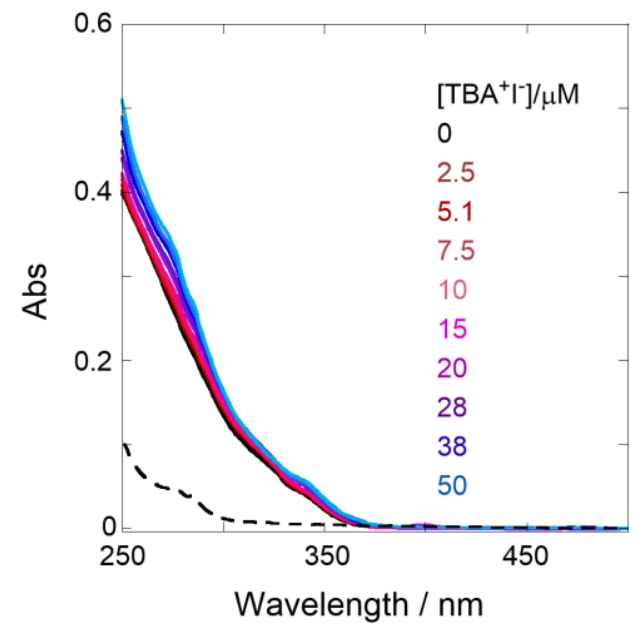

(b)

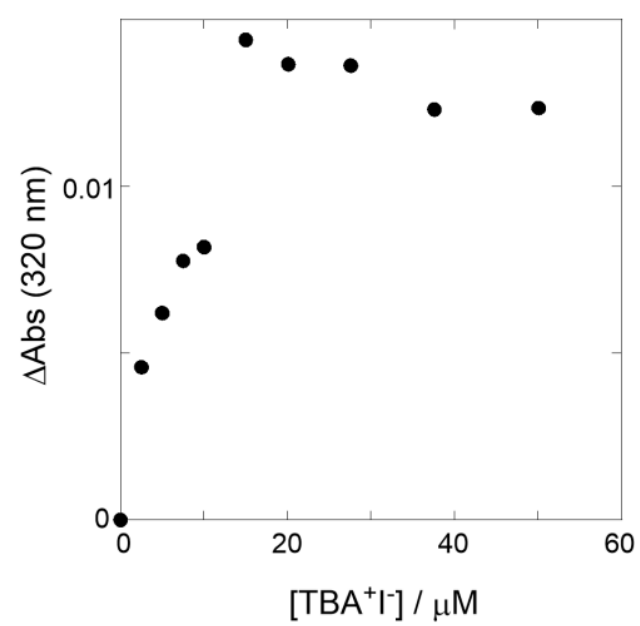

Figure S31. (a) UV/vis spectral changes of a chloroform solution of Mref (5.29 $\mu \mathrm{M}$, black) upon gradual addition of $\mathrm{TBA}^{+} \mathrm{I}^{-}\left(2.5-50 \mu \mathrm{M}\right.$, colored lines) at $25{ }^{\circ} \mathrm{C}$; the dotted line shows the UV spectrum of $\mathrm{TBA}^{+} \mathrm{I}^{-}$only. (b) Absorbance plots at $320 \mathrm{~nm}$ as a function of $\mathrm{TBA}^{+} \mathrm{I}^{-}$concentration at $25{ }^{\circ} \mathrm{C}$.

Discussion: With respect to the selectivity, based on the fluctuated Job plot and titration data of $\mathrm{TBA}^{+} \mathrm{T}^{-}$(Figure S30 in SI), some higher complex species besides the 1:1 complex of $\mathrm{TBA}^{+} \mathrm{T}^{-}$with $\mathbf{M}$ existed, suggesting the importance of each bond angle (o-, $\left.m^{-} v s p^{-}\right)$in the benzene core of the analyte. More importantly, in employing the corresponding reference host (Mref, Figure 2e) on behalf of $\mathbf{M}$ for the complexation experiment, the UV/vis titration data deviated from the 1:1 composite model (Figure S31 in SI), strongly indicating that a certain degree of fixation by the bithiophene scaffold was quite significant for this clipping complexation mode by the tweeze unit. 
(a)

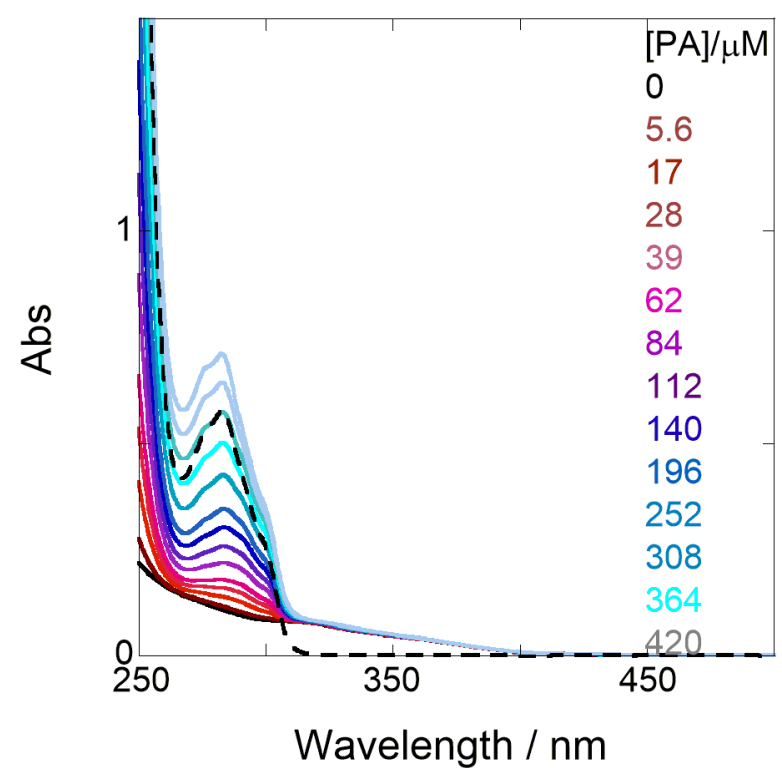

(b)

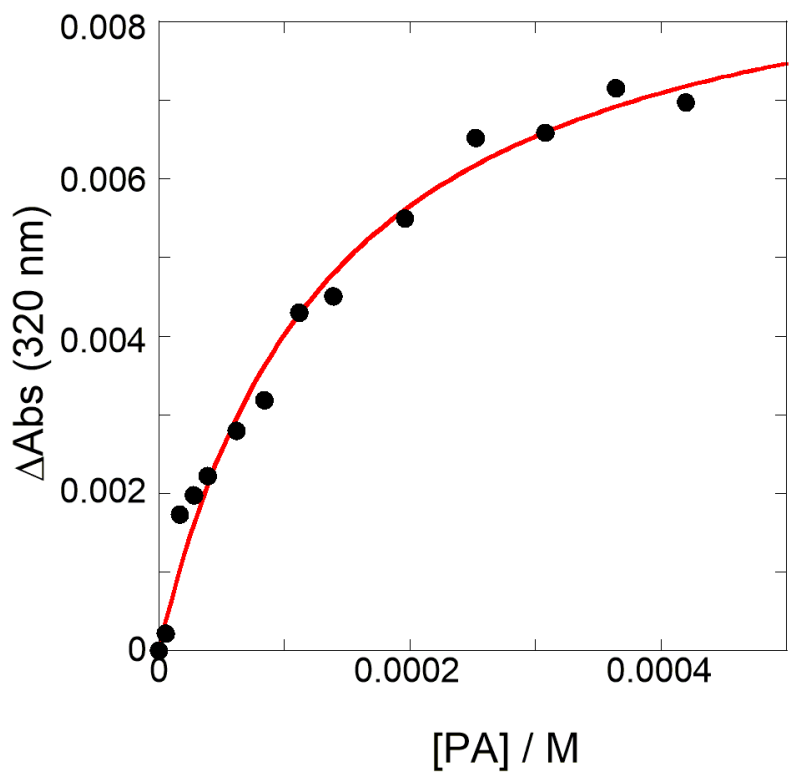

Figure S32. (a) UV/vis spectral changes of a chloroform solution ( $<2$ vol. $\%$ DMF) of $\mathbf{P}(5.54 \mu \mathrm{M}$ in monomer unit, black) upon gradual addition of PA $\left(5.6-420 \mu \mathrm{M}\right.$, colored lines) at $25^{\circ} \mathrm{C}$; the dotted line shows the UV spectrum of PA only. (b) Non-linear least-squares fitting, assuming 1:1 stoichiometry, to determine the binding constant at $25^{\circ} \mathrm{C}$.

(a)

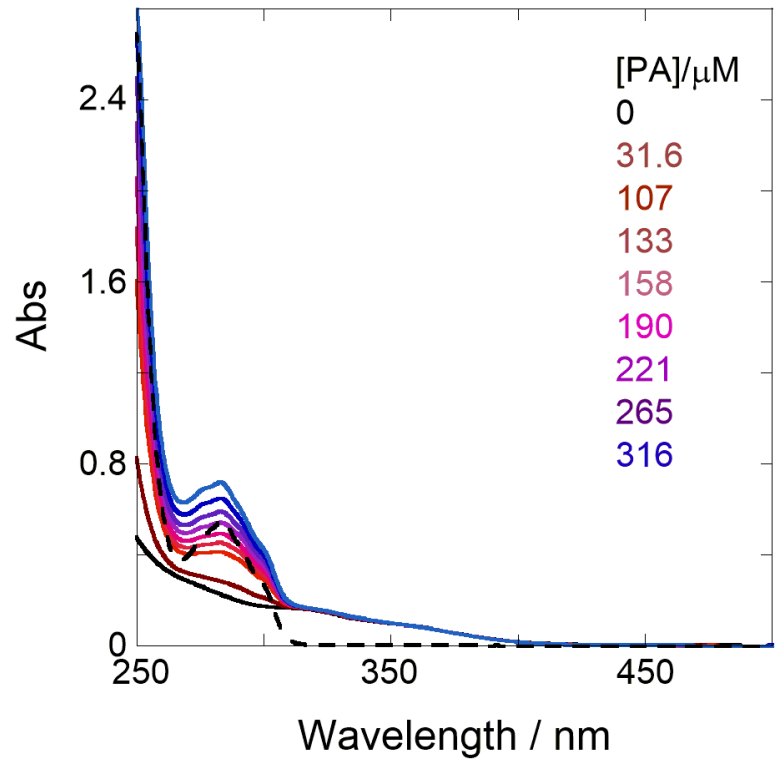

(b)

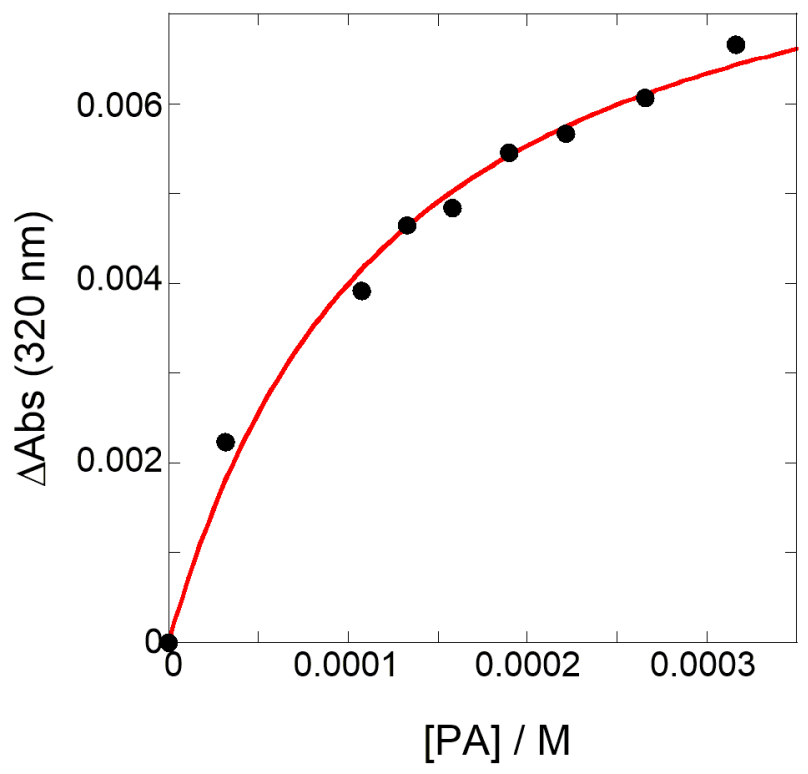

Figure S33. (a) UV/vis spectral changes of a chloroform solution ( $<2$ vol.\% DMF) of $\mathbf{P}(10.4 \mu \mathrm{M}$ in monomer unit, black) upon gradual addition of PA $\left(31.6-316 \mu \mathrm{M}\right.$, colored lines) at $30{ }^{\circ} \mathrm{C}$; the dotted line shows the UV spectrum of PA only. (b) Non-linear least-squares fitting, assuming 1:1 stoichiometry, to determine the binding constant at $30{ }^{\circ} \mathrm{C}$. 
(a)

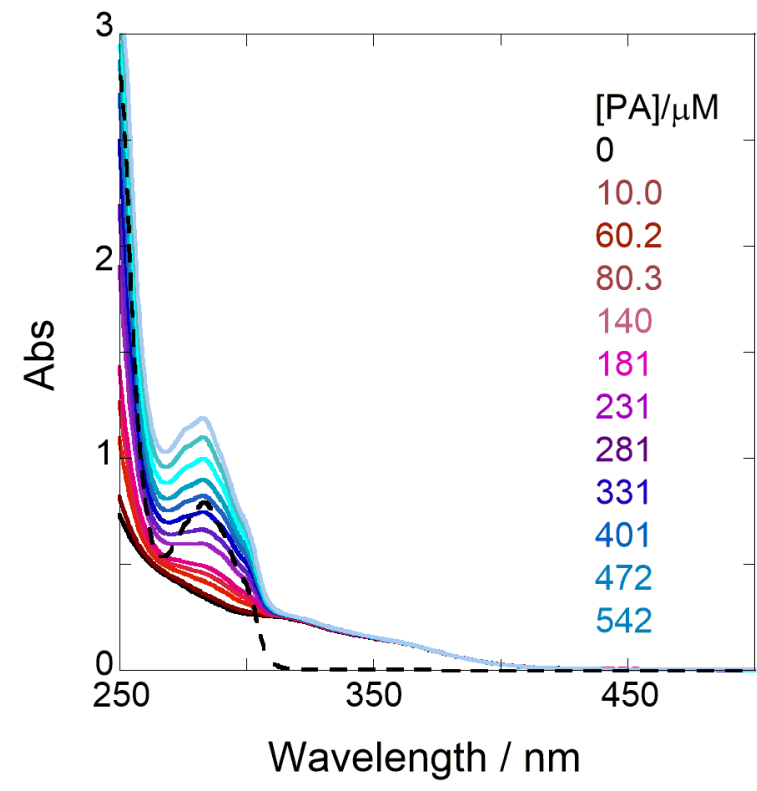

(b)

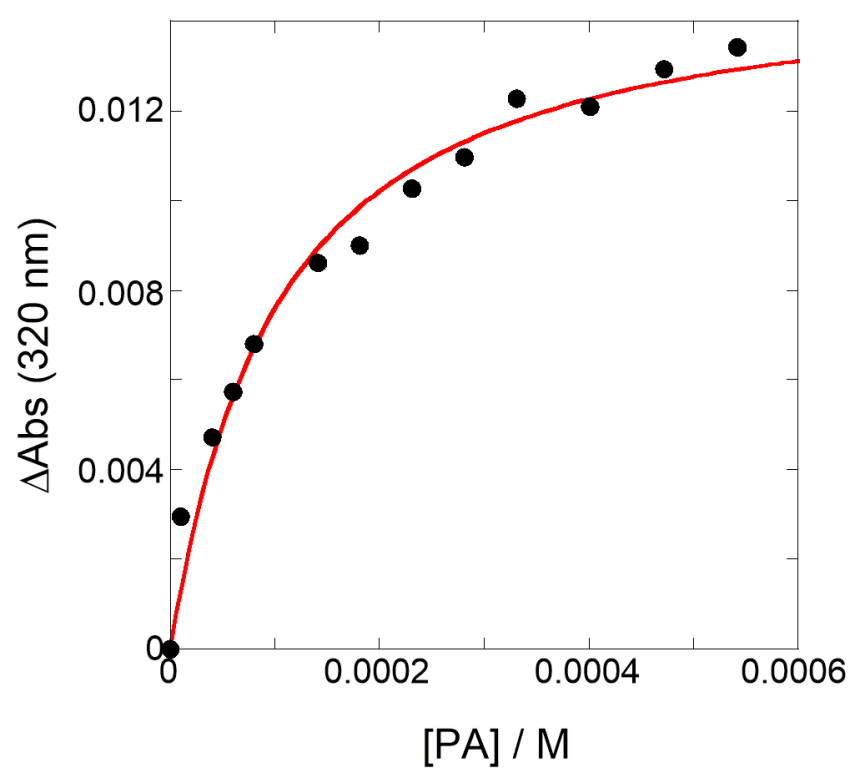

Figure S34. (a) UV/vis spectral changes of a chloroform solution ( $<2$ vol.\% DMF) of $\mathbf{P}(15.5 \mu \mathrm{M}$ in monomer unit, black) upon gradual addition of PA (10.0-542 $\mu \mathrm{M}$, colored lines) at $35{ }^{\circ} \mathrm{C}$; the dotted line shows the UV spectrum of PA only. (b) Non-linear least-squares fitting, assuming 1:1 stoichiometry, to determine the binding constant at $35{ }^{\circ} \mathrm{C}$.

(a)

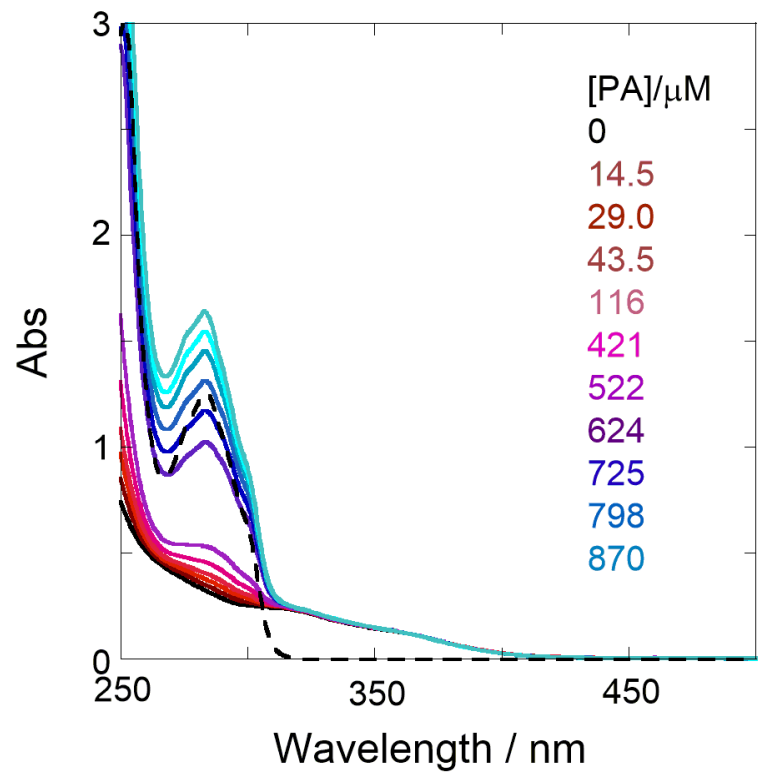

(b)

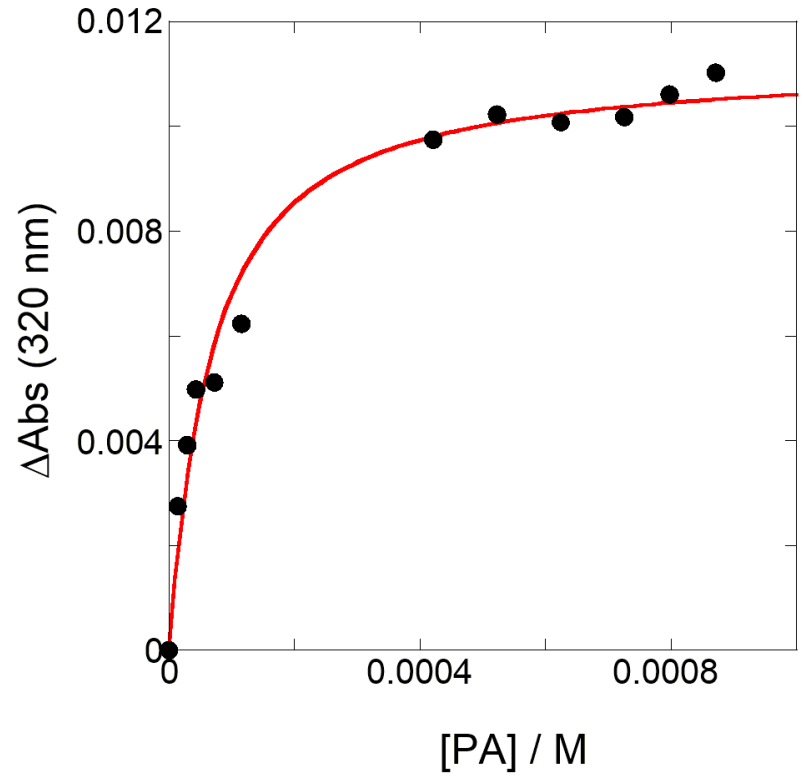

Figure S35. (a) UV/vis spectral changes of a chloroform solution ( $<2$ vol. $\%$ DMF $)$ of $\mathbf{P}(15.5 \mu \mathrm{M}$ in monomer unit, black) upon gradual addition of PA $(14.5-870 \mu \mathrm{M}$, colored lines $)$ at $40{ }^{\circ} \mathrm{C}$; the dotted line shows the UV spectrum of PA only. (b) Non-linear least-squares fitting, assuming 1:1 stoichiometry, to determine the binding constant at $40{ }^{\circ} \mathrm{C}$. 
(a)

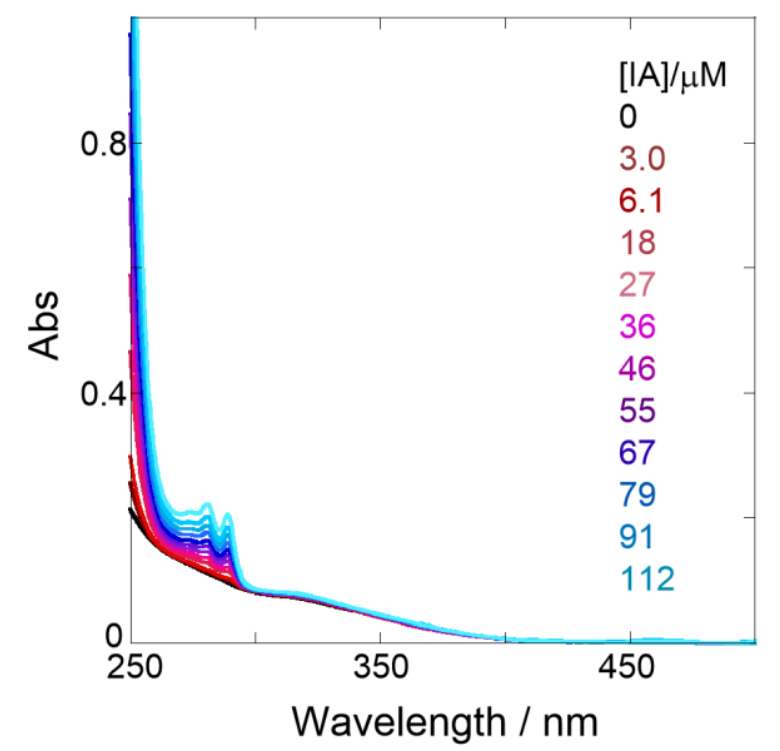

(c)

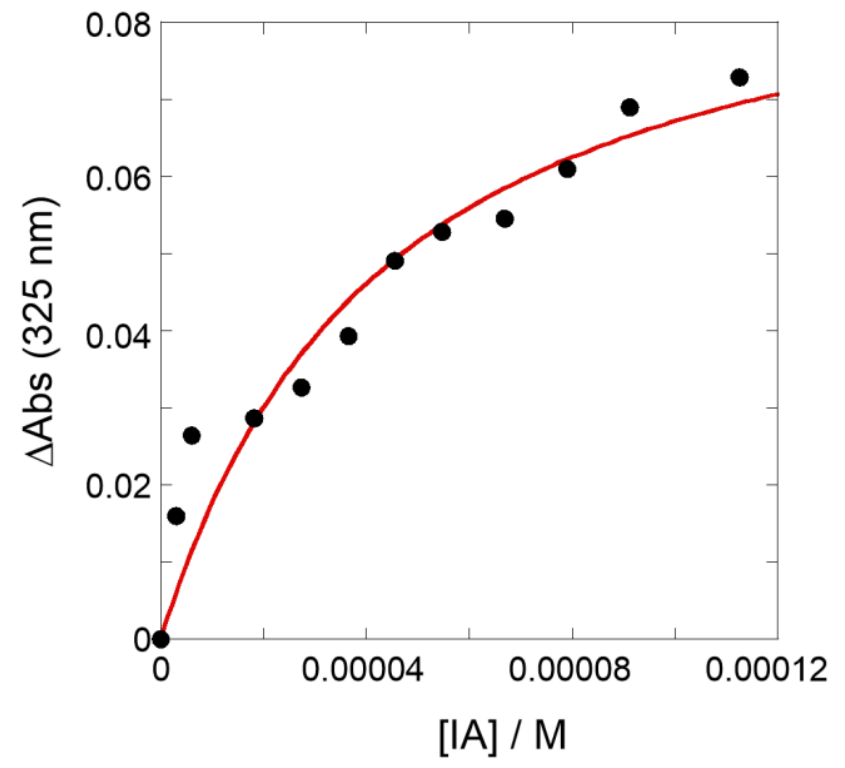

(b)

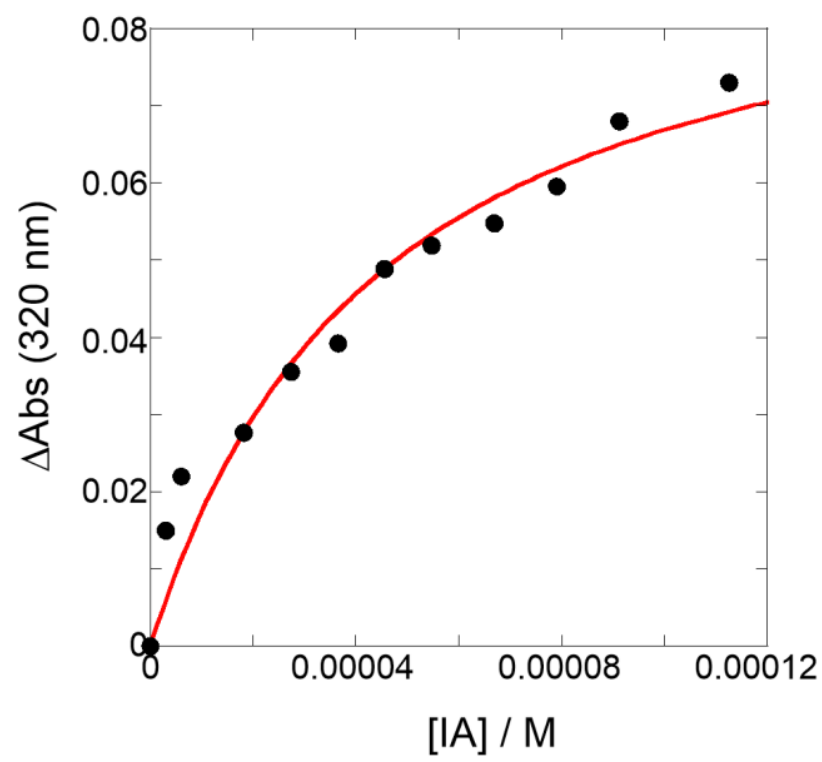

(d)

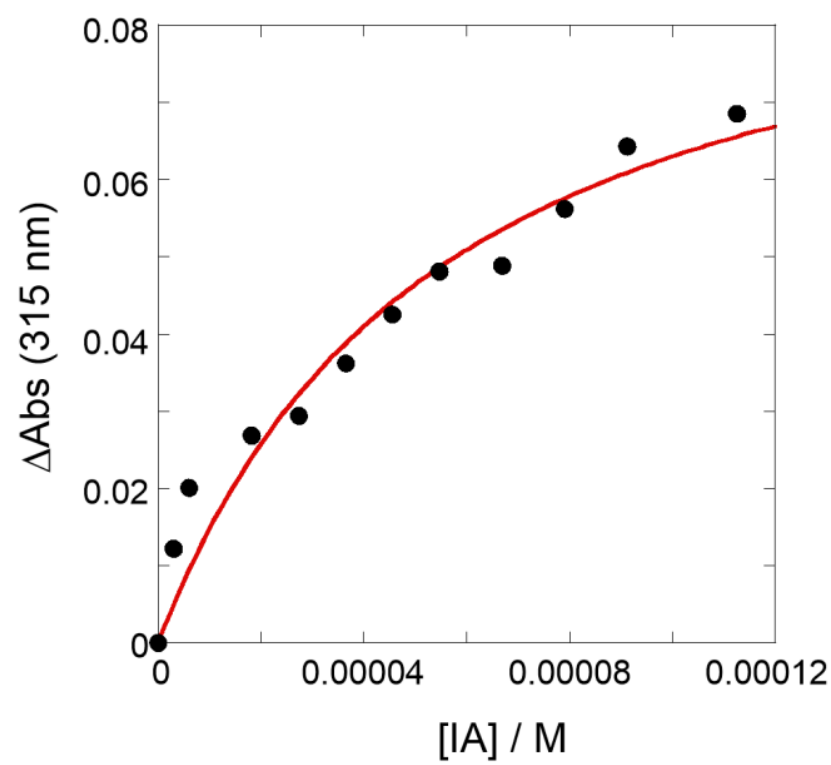

Figure S36. (a) UV/vis spectral changes of a chloroform solution ( $<2$ vol.\% DMF) of $\mathbf{P}(5.03 \mu \mathrm{M}$ in monomer unit, black) upon gradual addition of IA (3.0-112 $\mu \mathrm{M}$, colored lines) at $25{ }^{\circ} \mathrm{C}$. (b) Non-linear least-squares fitting, assuming 1:1 stoichiometry, to determine the binding constant monitored (b) at $320 \mathrm{~nm}\left(K=24600 \pm 7200 \mathrm{M}^{-1}\right)$, (c) at $325 \mathrm{~nm}\left(K=25300 \pm 9300 \mathrm{M}^{-1}\right)$, and (d) at $315 \mathrm{~nm}\left(K=20000 \pm 6100 \mathrm{M}^{-1}\right)$ at $25^{\circ} \mathrm{C}$. 
(a)

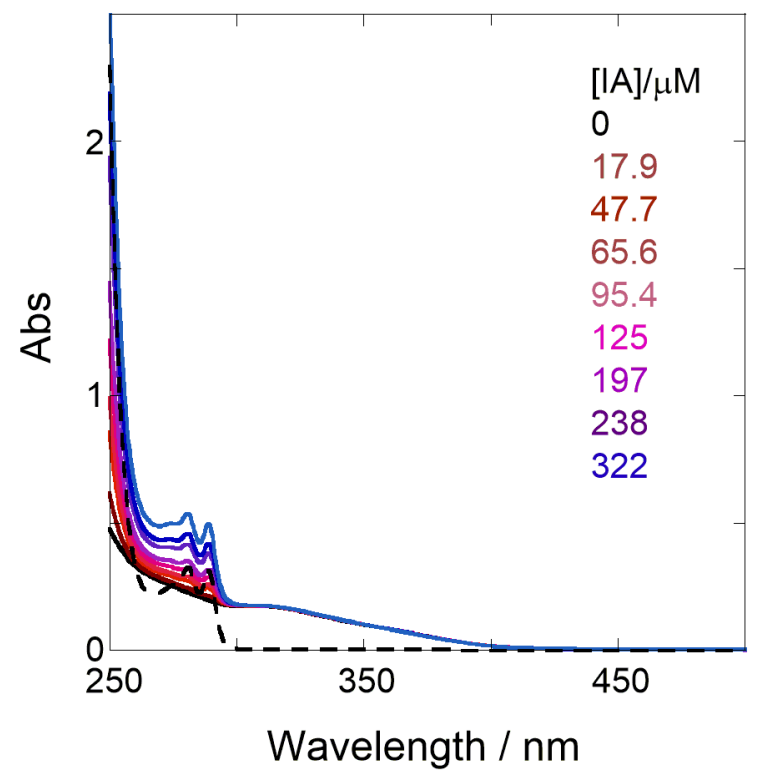

(b)

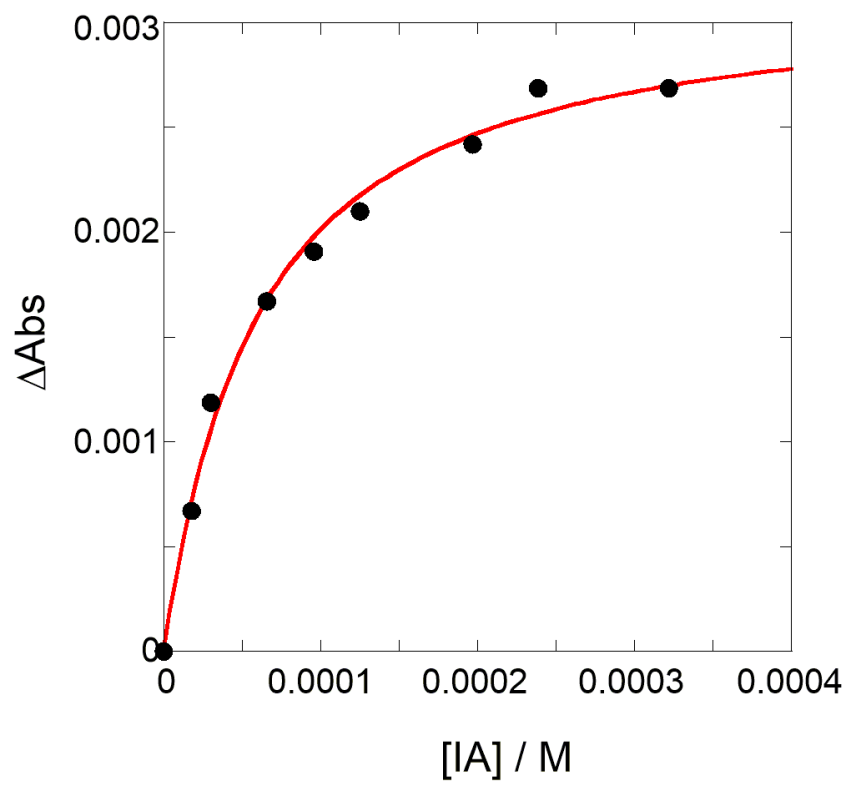

Figure S37. (a) UV/vis spectral changes of a chloroform solution ( $<2$ vol.\% DMF) of $\mathbf{P}(10.1 \mu \mathrm{M}$ in monomer unit, black) upon gradual addition of IA (17.9-322 $\mu \mathrm{M}$, colored lines) at $30{ }^{\circ} \mathrm{C}$; the dotted line shows the UV spectrum of IA only. (b) Non-linear least-squares fitting, assuming 1:1 stoichiometry, to determine the binding constant at $30{ }^{\circ} \mathrm{C}$.

(a)

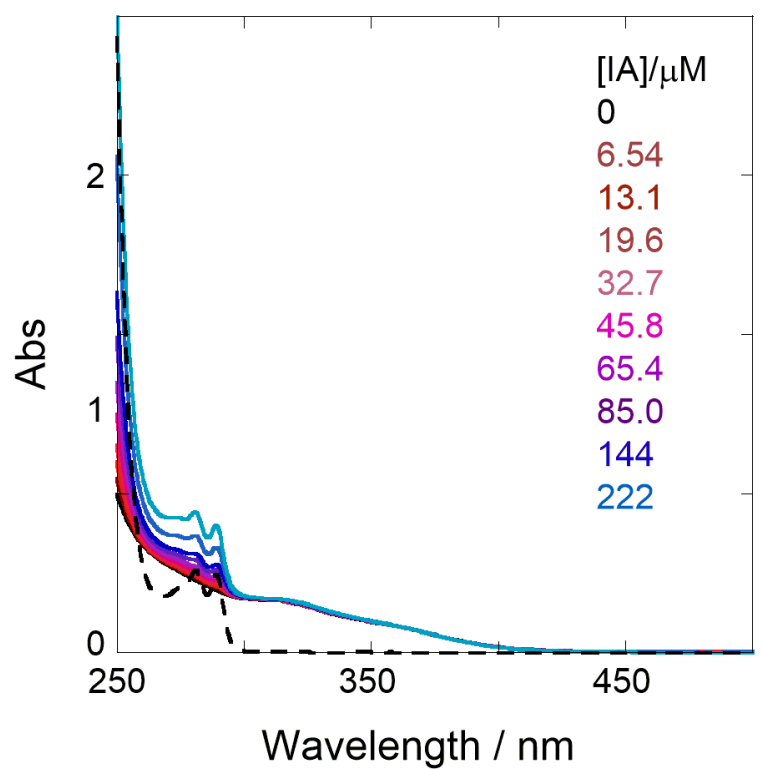

(b)

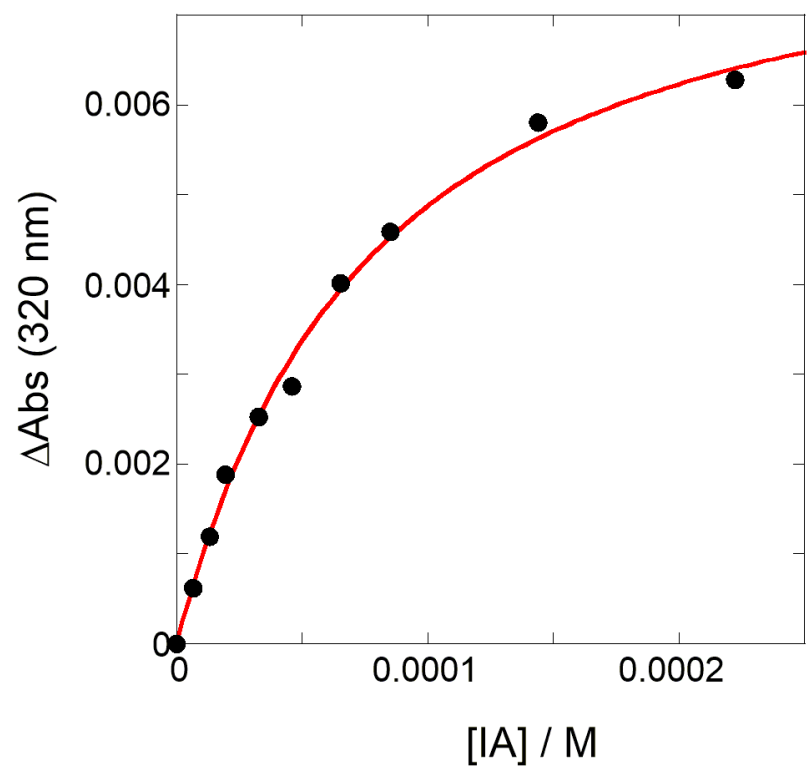

Figure S38. (a) UV/vis spectral changes of a chloroform solution $(<2$ vol.\% DMF) of $\mathbf{P}(10.4 \mu \mathrm{M}$ in monomer unit, black) upon gradual addition of IA $\left(6.54-222 \mu \mathrm{M}\right.$, colored lines) at $35^{\circ} \mathrm{C}$; the dotted line shows the UV spectrum of IA only. (b) Non-linear least-squares fitting, assuming 1:1 stoichiometry, to determine the binding constant at $35^{\circ} \mathrm{C}$. 
(a)

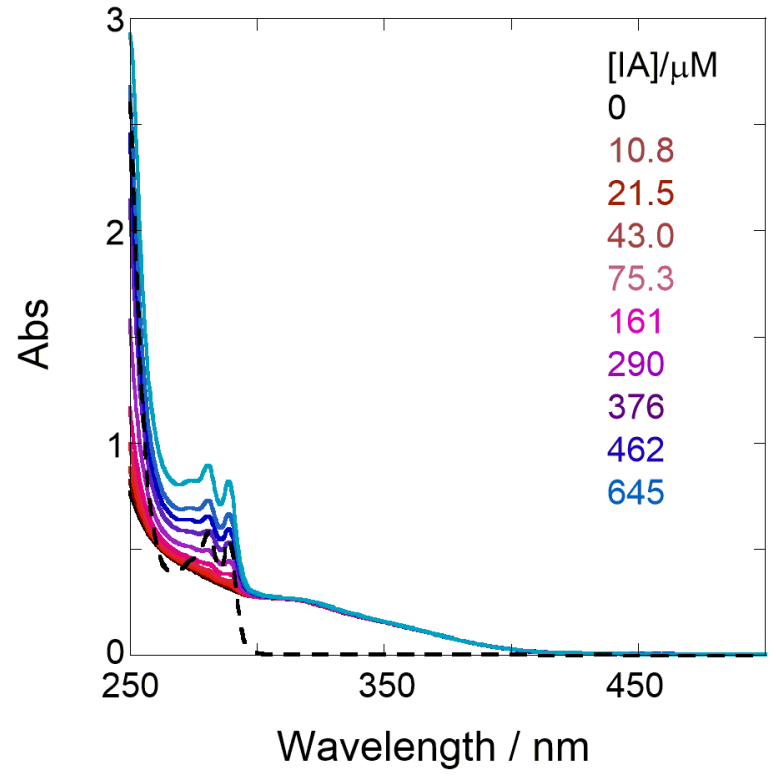

(b)

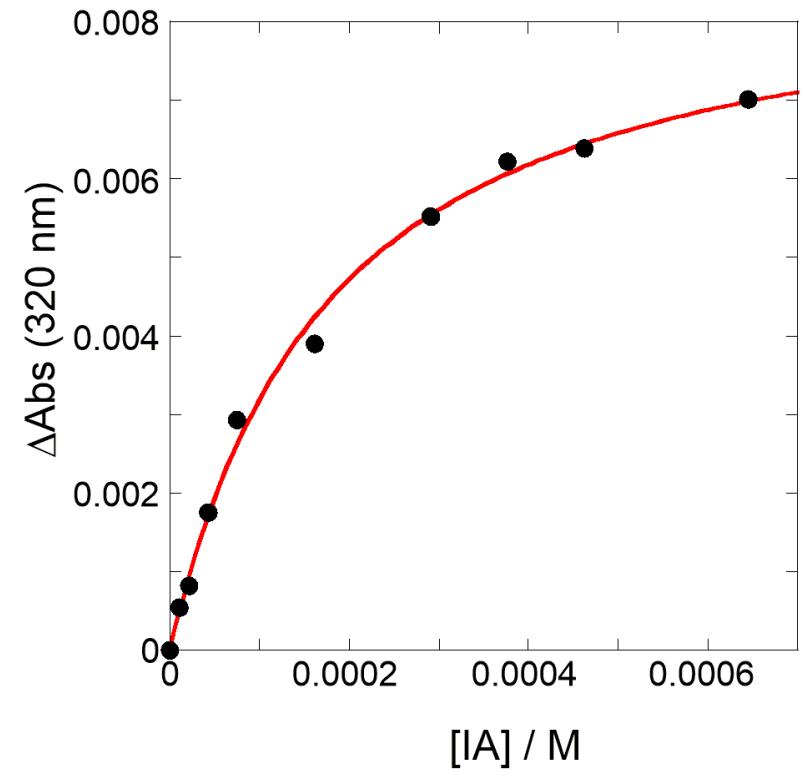

Figure S39. (a) UV/vis spectral changes of a chloroform solution ( $<2$ vol.\% DMF) of $\mathbf{P}(16.5 \mu \mathrm{M}$ in monomer unit, black) upon gradual addition of IA (10.8-645 $\mu \mathrm{M}$, colored lines) at $40{ }^{\circ} \mathrm{C}$; the dotted line shows the UV spectrum of IA only. (b) Non-linear least-squares fitting, assuming 1:1 stoichiometry, to determine the binding constant at $40{ }^{\circ} \mathrm{C}$.

(a)

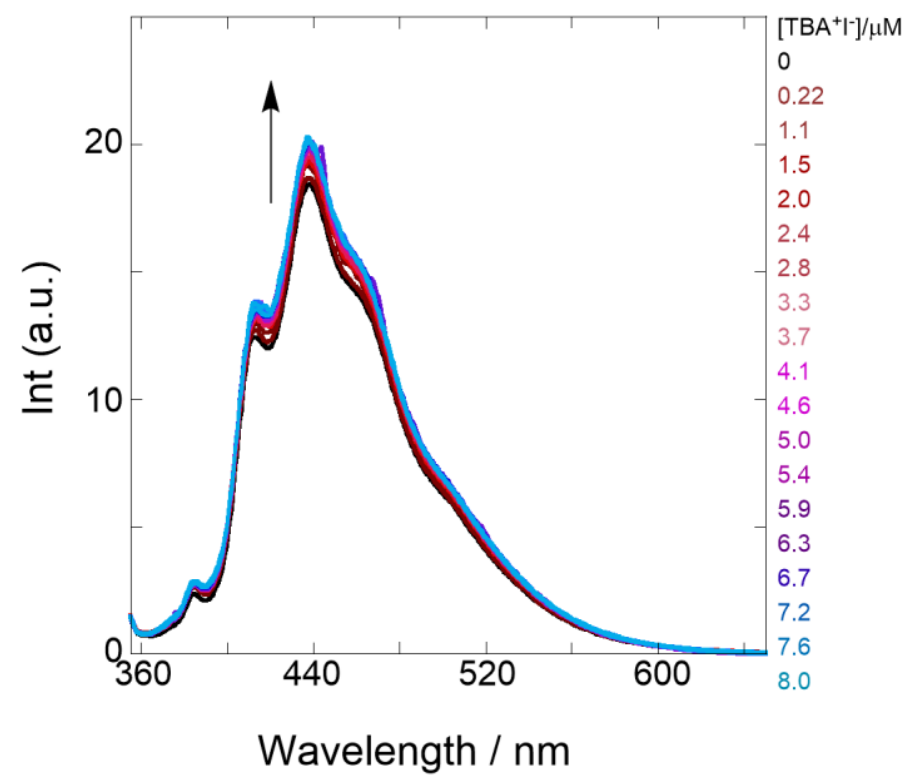

(b)

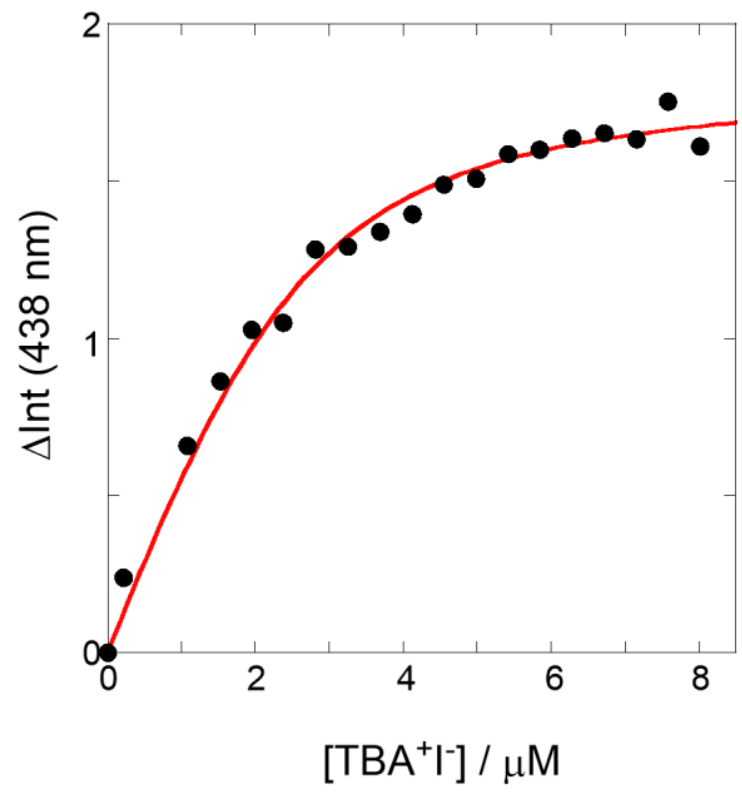

Figure S40. (a) Fluorescence spectral changes, excited at $345 \mathrm{~nm}$ (isosbestic point), of a chloroform solution of $\mathbf{P}\left(2.51 \mu \mathrm{M}\right.$ in monomer unit, black) upon gradual addition of $\mathrm{TBA}^{+} \mathrm{I}^{-}(0.22-8.0 \mu \mathrm{M}$, colored lines) at $25{ }^{\circ} \mathrm{C}$. (b) Non-linear least-squares fitting, assuming 1:1 stoichiometry, to determine the binding constant at $25^{\circ} \mathrm{C}$. 
Discussion: The fluorescence increase is highly likely to originate from a suppression of photoinduced electron transfer (PET)-based quenching from the amines in the thiourea moieties to the polythiophene reporter upon complexation of the dicarboxylates. As shown in Figure S41a, the fluorescence lifetime decays of the sensor $\mathbf{P}$ were reasonably fitted to a sum of two exponential functions to give two lifetimes of $0.3 \mathrm{~ns}$ and $1.3 \mathrm{~ns}$ (Table S2), both of which may be assigned to excited PT species with long and short conjugation lengths. These values were almost unchanged upon the complexation of $\mathrm{TBA}^{+} \mathrm{I}^{-}$, indicating that the suppression of the "intra-complex" fluorescence quenching through PET was extremely fast, rather static. The superimposable normalized fluorescence spectra of Figure S41b also reinforced this fluorescence enhancement process.

Table S2. Fluorescence Lifetimes of $P$ in the Absence and Presence of Tetrabutylammonium Isophthalate $^{a}$

\begin{tabular}{|c|c|c|c|c|c|c|}
\hline Solution & $n^{b}$ & $\tau_{1} / \mathrm{ns}$ & $A_{1}$ & $\tau_{2} / \mathrm{ns}$ & $A_{2}$ & $\chi^{2}$ \\
\hline $\mathbf{P}$ & 2 & 0.3 & 0.30 & 1.3 & 0.70 & 1.1 \\
\hline $\mathbf{P}+\mathrm{TBA}^{+} \mathrm{I}^{-}$ & 2 & 0.2 & 0.30 & 1.3 & 0.70 & 1.3 \\
\hline
\end{tabular}

${ }^{a}$ Fluorescence lifetime $\left(\tau_{1} / \mathrm{ns}\right)$ and relative abundance $\left(A_{\mathrm{i}}\right)$ of each component were determined by the single-photon-counting method in a non-degassed solution at room temperature; $[\mathbf{P}]=2.51 \mu \mathrm{M}$ in monomer unit, $\left[\mathrm{TBA}^{+} \mathrm{I}^{-}\right]=8.0 \mu \mathrm{M} .{ }^{b}$ Number of components.

(a)

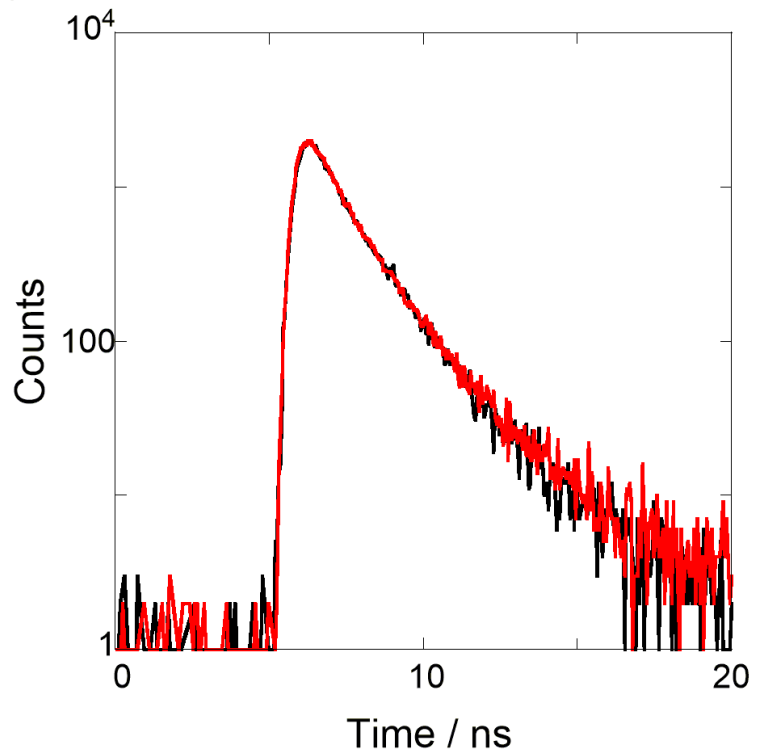

(b)

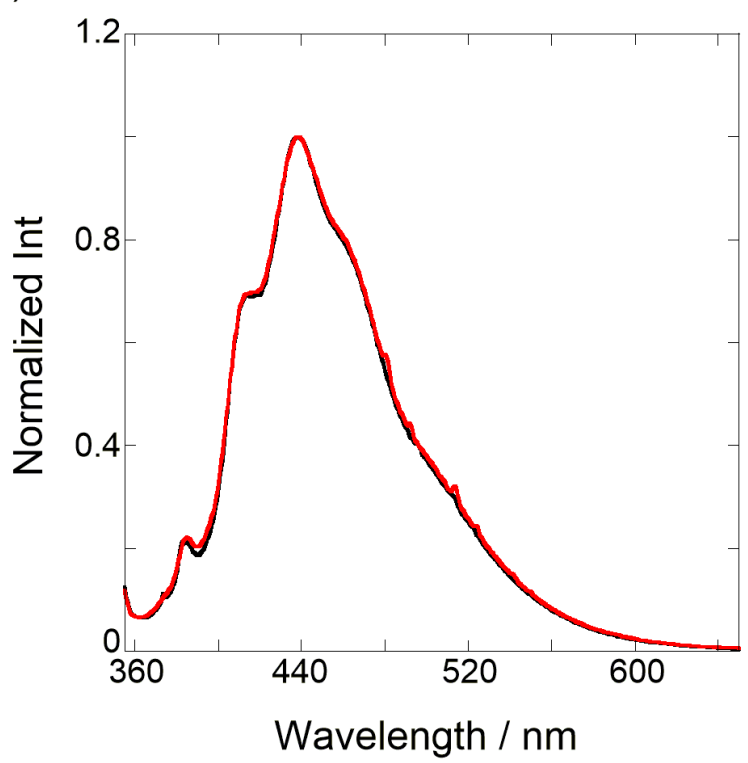

Figure S41. (a) Time-correlated fluorescence decays $\left(\lambda_{\mathrm{ex}} 340 \mathrm{~nm}, \lambda_{\mathrm{em}} 437 \mathrm{~nm}\right)$ and (b) normalized fluorescence spectra $\left(\lambda_{\mathrm{ex}} 345 \mathrm{~nm}\right)$ of a chloroform solution of $\mathbf{P}(2.51 \mu \mathrm{M}$ in monomer unit) in the absence (black) and presence (red) of $\mathrm{TBA}^{+} \mathrm{I}^{-}(8.0 \mu \mathrm{M})$ at room temperature. 
(a)

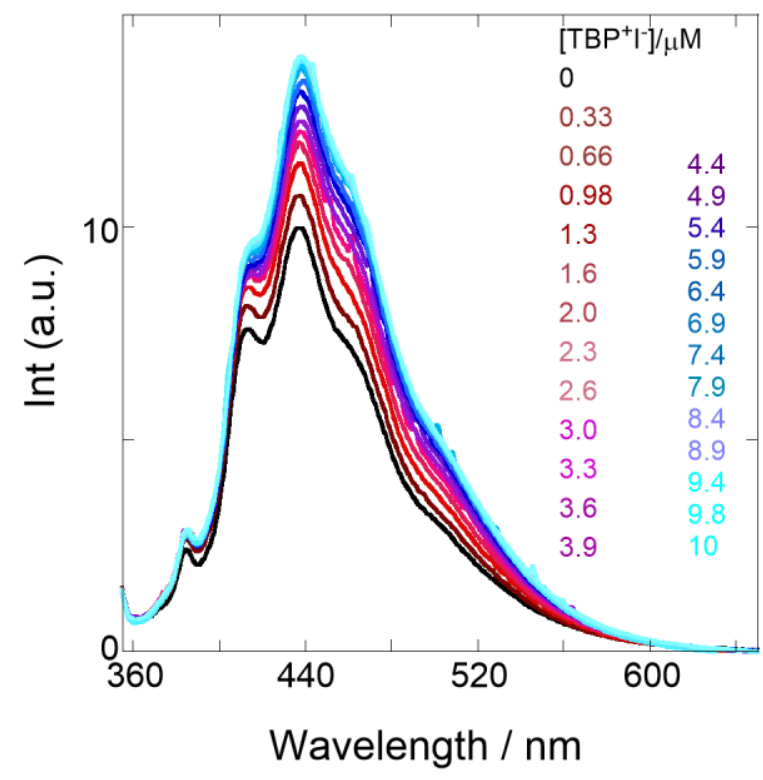

(b)

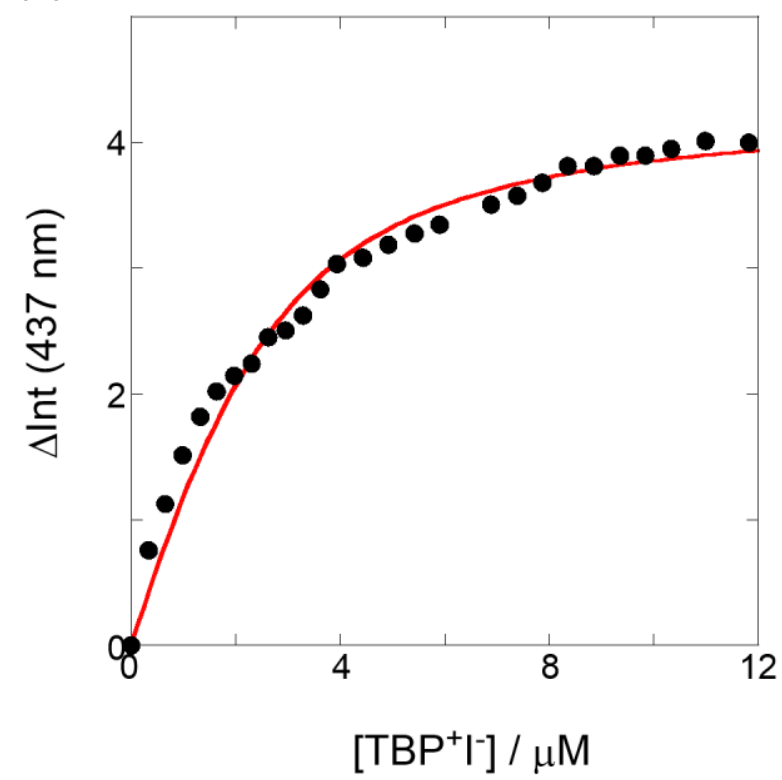

Figure S42. (a) Fluorescence spectral changes, excited at $345 \mathrm{~nm}$ (isosbestic point), of a chloroform solution of $\mathbf{P}\left(2.38 \mu \mathrm{M}\right.$ in monomer unit, black) upon gradual addition of $\mathrm{TBP}^{+} \mathrm{I}^{-}(0.33-10 \mu \mathrm{M}$, colored lines) at $25{ }^{\circ} \mathrm{C}$. (b) Non-linear least-squares fitting, assuming 1:1 stoichiometry, to determine the binding constant at $25^{\circ} \mathrm{C}$.

Table S3. Binding Constants $(K)$ and Thermodynamic Parameters for 1:1 Complexation of the Analytes with the Monomer (M) and Polythiophene (P) Sensors in $\mathrm{CHCl}^{a}$

\begin{tabular}{|c|c|c|c|c|c|c|}
\hline Host & Guest & Temp. $/{ }^{\circ} \mathrm{C}$ & $K / \mathrm{M}^{-1 b}$ & $\Delta H^{\circ} / \mathrm{kJ} \mathrm{mol}^{-1}$ & $T \Delta S^{\circ} / \mathrm{kJ} \mathrm{mol}^{-1}$ & $\Delta G^{\circ} / \mathrm{kJ} \mathrm{mol}^{-1}$ \\
\hline \multirow[t]{12}{*}{$\mathbf{M}$} & PA & 15 & $3250 \pm 488$ & & & \\
\hline & & 20 & $1060 \pm 144$ & & & \\
\hline & & 25 & $511 \pm 17.5$ & -90.8 & -74.6 & -16.2 \\
\hline & & 35 & $256 \pm 25.0$ & & & \\
\hline & IA & 15 & $11000 \pm 1230$ & & & \\
\hline & & 25 & $4350 \pm 245$ & -88.4 & -68.2 & -20.2 \\
\hline & & 30 & $1690 \pm 197$ & & & \\
\hline & & 35 & $1060 \pm 54.0$ & & & \\
\hline & $\mathrm{TBA}^{+} \mathrm{I}^{-}$ & 20 & $174000 \pm 32200$ & & & \\
\hline & & 25 & $159000 \pm 11300$ & -22.1 & 7.42 & -29.5 \\
\hline & & 30 & $130000 \pm 24500$ & & & \\
\hline & & 35 & $114000 \pm 18500$ & & & \\
\hline
\end{tabular}




\begin{tabular}{|c|c|c|c|c|c|c|}
\hline & $\mathrm{TBP}^{+} \mathrm{I}^{-}$ & 25 & $198000 \pm 39600$ & \multirow[t]{3}{*}{-15.2} & \multirow[t]{3}{*}{15.0} & \multirow[t]{3}{*}{-30.2} \\
\hline & & 35 & $152000 \pm 39500$ & & & \\
\hline & & 40 & $151000 \pm 25400$ & & & \\
\hline \multirow[t]{10}{*}{$\mathbf{P}$} & PA & 25 & $7540 \pm 1020$ & \multirow[t]{4}{*}{40.6} & \multirow[t]{4}{*}{62.5} & \multirow[t]{4}{*}{-21.9} \\
\hline & & 30 & $8650 \pm 1480$ & & & \\
\hline & & 35 & $10800 \pm 1720$ & & & \\
\hline & & 40 & $16800 \pm 2590$ & & & \\
\hline & \multirow[t]{4}{*}{ IA } & 25 & $24600 \pm 7230$ & \multirow[t]{4}{*}{-69.3} & \multirow[t]{4}{*}{-43.9} & \multirow[t]{4}{*}{-25.4} \\
\hline & & 30 & $18700 \pm 2180$ & & & \\
\hline & & 35 & $14400 \pm 1330$ & & & \\
\hline & & 40 & $6020 \pm 607$ & & & \\
\hline & $\mathrm{TBA}^{+} \mathrm{I}^{-}$ & 25 & $1780000 \pm 355000^{c}$ & $d$ & $d$ & -35.7 \\
\hline & $\mathrm{TBP}^{+} \mathrm{I}^{-}$ & 25 & $1070000 \pm 164000^{c}$ & $d$ & $d$ & -34.4 \\
\hline & $\begin{array}{l}\text { ation ex } \\
\text { mproved } \\
\text { JV/vis t } \\
\text { omer uni }\end{array}$ & & $\begin{array}{l}\text { for phthalic acids we } \\
\text { The entropy and Gi } \\
\text { aless noted otherwise } \\
\text { ined via fluorescence }\end{array}$ & $\begin{array}{l}\mathrm{d} \text { in } \mathrm{CH} \\
\text { changes }\end{array}$ & $\begin{array}{l}\text { aining I } \\
\text { are lis } \\
\mathbf{P} \text { were } \\
\text { ing to la }\end{array}$ & $\begin{array}{l}2 \text { vol.\%) } \\
\text { etermined } \\
\text { ined as a } \\
\text { ues. }\end{array}$ \\
\hline
\end{tabular}




\section{Comparison Titration Curves}

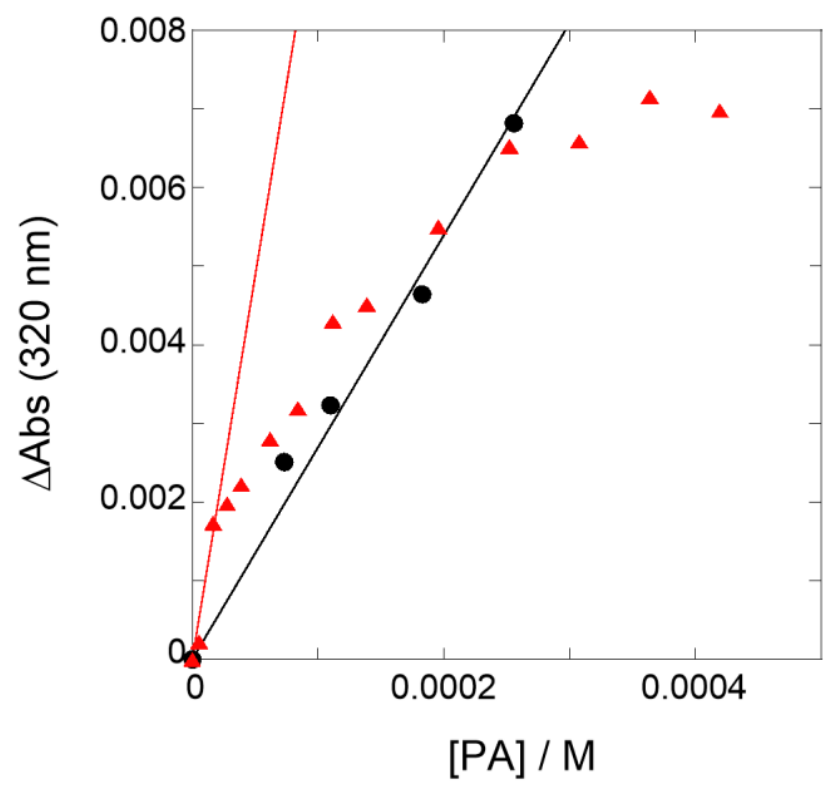

Figure S43. UV/vis titration data of $\mathbf{M}$ (black circle) or $\mathbf{P}$ (red triangle) upon gradual addition of PA at $25{ }^{\circ} \mathrm{C}$ : red slope 96 ; black slope 27. 


\section{Waveform Separation Spectra}

(a)

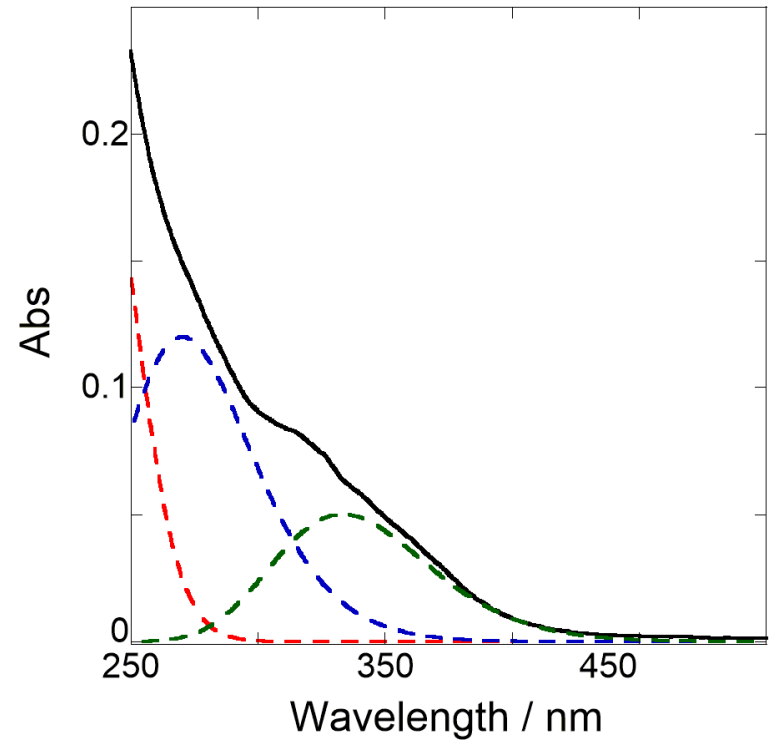

(b)

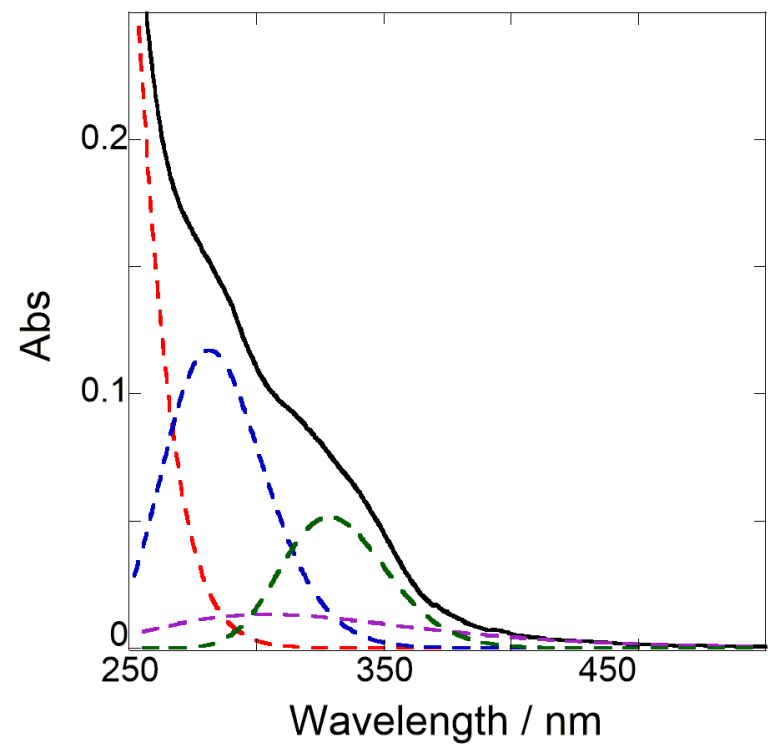

Figure S44. Results of waveform separation (dotted line) and UV/vis (solid line) spectra of a chloroform solution of $\mathbf{P}\left(5.03 \mu \mathrm{M}\right.$ in monomer unit) in the absence (a) and presence (b) of $\mathrm{TBA}^{+} \mathrm{I}^{-}$ $(18 \mu \mathrm{M})$. 


\section{Theoretical Calculations of Oligomer Analogs}

(a)

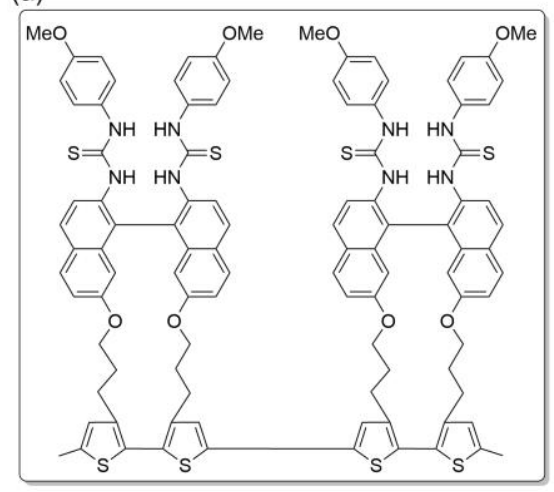

(b)

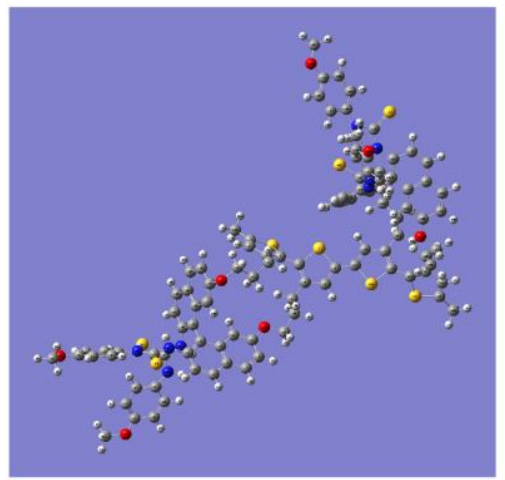

(c)

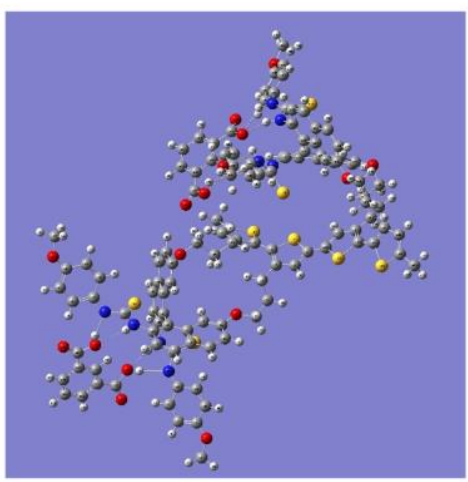

Figure S45. (a) Chemical structure of the 4-mer analog; the oxyethylene chains and the chloro-groups were omitted and both ends at $\alpha$ positions were methylated for simplified calculations. Optimized structures of the 4-mer analog using Gaussian 16 calculation (HF/6-31G) in the absence (b) and presence (c) of isophthalate; counter cation was omitted for clarity. The number of imaginary frequencies was not involved during the calculation due to a high running cost.

\section{Summary of Structure Optimization}

the 4-mer analog

Z-matrices

$\begin{array}{lrrrrrrrrrr}\mathrm{C} & 0 & 1 & 0 & 1 & 0 & 1 & 0 & 0 & 0 & \# \\ \mathrm{C} & 1.368407 & 1 & 0 & 1 & 0 & 1 & 1 & 0 & 0 & \# \\ \mathrm{C} & 1.435248 & 1 & 115.6902 & 1 & 0 & 1 & 2 & 1 & 0 & \# \\ \mathrm{C} & 1.372136 & 1 & 113.6339 & 1 & -1.20032 & 1 & 3 & 2 & 1 & \# \\ \mathrm{~S} & 1.820114 & 1 & 110.1952 & 1 & 0.926722 & 1 & 1 & 2 & 3 & \# \\ \mathrm{C} & 1.441936 & 1 & 130.4967 & 1 & -179.535 & 1 & 4 & 3 & 2 & \# \\ \mathrm{C} & 1.371006 & 1 & 130.1385 & 1 & 62.42603 & 1 & 6 & 4 & 3 & \# \\ \mathrm{C} & 1.443844 & 1 & 113.178 & 1 & 178.1936 & 1 & 7 & 6 & 4 & \# \\ \mathrm{C} & 1.358762 & 1 & 115.727 & 1 & -0.90404 & 1 & 8 & 7 & 6 & \# \\ \mathrm{~S} & 1.811894 & 1 & 110.5874 & 1 & 0.37791 & 1 & 9 & 8 & 7 & \# \\ \mathrm{C} & 6.286694 & 1 & 116.3149 & 1 & 175.5128 & 1 & 3 & 2 & 1 & \# \\ \mathrm{C} & 1.376333 & 1 & 152.9448 & 1 & 118.353 & 1 & 11 & 3 & 2 & \# \\ \mathrm{C} & 1.414865 & 1 & 121.4495 & 1 & 66.56252 & 1 & 12 & 11 & 3 & \# \\ \mathrm{C} & 1.4368 & 1 & 118.8964 & 1 & 1.613794 & 1 & 13 & 12 & 11 & \# \\ \mathrm{C} & 1.418246 & 1 & 118.4546 & 1 & -1.7529 & 1 & 14 & 13 & 12 & \# \\ \mathrm{C} & 1.384474 & 1 & 121.2312 & 1 & -0.37909 & 1 & 15 & 14 & 13 & \# \\ \mathrm{C} & 1.419155 & 1 & 122.3236 & 1 & -179.032 & 1 & 13 & 12 & 11 & \# \\ \mathrm{C} & 1.368821 & 1 & 121.6935 & 1 & -179.64 & 1 & 17 & 13 & 12 & \#\end{array}$




\begin{tabular}{|c|c|c|c|c|c|c|c|c|c|c|}
\hline $\mathrm{C}$ & 1.426007 & 1 & 120.6932 & 1 & 0.535531 & 1 & 18 & 17 & 13 & \# \\
\hline $\mathrm{C}$ & 1.403957 & 1 & 119.3638 & 1 & 0.257893 & 1 & 19 & 18 & 17 & \# \\
\hline $\mathrm{C}$ & 4.353578 & 1 & 118.5596 & 1 & -117.28 & 1 & 20 & 19 & 18 & \# \\
\hline $\mathrm{C}$ & 1.3781 & 1 & 0.533358 & 1 & 177.6848 & 1 & 21 & 20 & 19 & \# \\
\hline $\mathrm{C}$ & 1.418693 & 1 & 120.8142 & 1 & 102.6592 & 1 & 22 & 21 & 20 & \# \\
\hline $\mathrm{C}$ & 1.434569 & 1 & 118.9597 & 1 & 0.242463 & 1 & 23 & 22 & 21 & \# \\
\hline $\mathrm{C}$ & 1.419941 & 1 & 118.7554 & 1 & -2.25341 & 1 & 24 & 23 & 22 & \# \\
\hline $\mathrm{C}$ & 1.373532 & 1 & 120.9377 & 1 & 2.070498 & 1 & 25 & 24 & 23 & \# \\
\hline $\mathrm{C}$ & 1.434604 & 1 & 121.9865 & 1 & 178.8731 & 1 & 23 & 22 & 21 & \# \\
\hline $\mathrm{C}$ & 1.391776 & 1 & 119.2901 & 1 & -177.031 & 1 & 27 & 23 & 22 & \# \\
\hline $\mathrm{C}$ & 1.419241 & 1 & 121.2899 & 1 & -0.67421 & 1 & 28 & 27 & 23 & \# \\
\hline $\mathrm{C}$ & 1.370083 & 1 & 119.9841 & 1 & -0.86404 & 1 & 29 & 28 & 27 & \# \\
\hline $\mathrm{C}$ & 1.512873 & 1 & 121.6027 & 1 & 176.0817 & 1 & 3 & 2 & 1 & \# \\
\hline $\mathrm{O}$ & 1.389351 & 1 & 116.3893 & 1 & -176.715 & 1 & 16 & 15 & 14 & \# \\
\hline $\mathrm{O}$ & 1.398745 & 1 & 118.5943 & 1 & 75.56607 & 1 & 21 & 20 & 19 & \# \\
\hline $\mathrm{N}$ & 1.395859 & 1 & 122.3421 & 1 & 174.941 & 1 & 19 & 18 & 17 & \# \\
\hline $\mathrm{N}$ & 1.423015 & 1 & 118.3032 & 1 & 176.1388 & 1 & 28 & 27 & 23 & \# \\
\hline $\mathrm{C}$ & 1.396754 & 1 & 131.1768 & 1 & 18.62276 & 1 & 34 & 19 & 18 & \# \\
\hline $\mathrm{C}$ & 1.375508 & 1 & 126.6563 & 1 & 132.5697 & 1 & 35 & 28 & 27 & \# \\
\hline $\mathrm{N}$ & 1.361841 & 1 & 109.6028 & 1 & -159.532 & 1 & 36 & 34 & 19 & \# \\
\hline$S$ & 1.726288 & 1 & 123.8637 & 1 & 22.19053 & 1 & 36 & 34 & 19 & \# \\
\hline $\mathrm{N}$ & 1.360622 & 1 & 112.3812 & 1 & 172.2677 & 1 & 37 & 35 & 28 & \# \\
\hline S & 1.743332 & 1 & 121.5216 & 1 & -7.61244 & 1 & 37 & 35 & 28 & \# \\
\hline $\mathrm{C}$ & 1.417398 & 1 & 132.2846 & 1 & -172.686 & 1 & 38 & 36 & 34 & \# \\
\hline $\mathrm{C}$ & 1.423464 & 1 & 132.9071 & 1 & 171.2026 & 1 & 40 & 37 & 35 & \# \\
\hline $\mathrm{C}$ & 1.398793 & 1 & 125.8368 & 1 & 12.24701 & 1 & 42 & 38 & 36 & \# \\
\hline $\mathrm{C}$ & 1.396562 & 1 & 120.4634 & 1 & 178.4298 & 1 & 44 & 42 & 38 & \# \\
\hline $\mathrm{C}$ & 1.394879 & 1 & 121.0035 & 1 & -0.01204 & 1 & 45 & 44 & 42 & \# \\
\hline $\mathrm{C}$ & 1.402882 & 1 & 118.7021 & 1 & -0.1797 & 1 & 46 & 45 & 44 & \# \\
\hline $\mathrm{C}$ & 1.382926 & 1 & 120.4808 & 1 & 0.176785 & 1 & 47 & 46 & 45 & \# \\
\hline $\mathrm{C}$ & 1.408733 & 1 & 116.04 & 1 & 172.9192 & 1 & 43 & 40 & 37 & \# \\
\hline $\mathrm{C}$ & 1.383164 & 1 & 121.1757 & 1 & 178.5292 & 1 & 49 & 43 & 40 & \# \\
\hline $\mathrm{C}$ & 1.402906 & 1 & 120.3942 & 1 & -0.05282 & 1 & 50 & 49 & 43 & \# \\
\hline $\mathrm{C}$ & 1.395807 & 1 & 118.6645 & 1 & 0.078683 & 1 & 51 & 50 & 49 & \# \\
\hline $\mathrm{C}$ & 1.395387 & 1 & 121.0211 & 1 & -0.00297 & 1 & 52 & 51 & 50 & \# \\
\hline $\mathrm{C}$ & 1.468291 & 1 & 119.4346 & 1 & -175.978 & 1 & 32 & 16 & 15 & \# \\
\hline $\mathrm{C}$ & 1.479073 & 1 & 115.1049 & 1 & 125.438 & 1 & 33 & 21 & 20 & \# \\
\hline $\mathrm{C}$ & 1.528013 & 1 & 106.198 & 1 & -163.705 & 1 & 54 & 32 & 16 & \# \\
\hline
\end{tabular}




\begin{tabular}{|c|c|c|c|c|c|c|c|c|c|c|}
\hline $\mathrm{C}$ & 1.536927 & 1 & 111.2873 & 1 & -74.5865 & 1 & 55 & 33 & 21 & $\#$ \\
\hline $\mathrm{C}$ & 1.51747 & 1 & 123.757 & 1 & 0.084229 & 1 & 7 & 6 & 4 & $\#$ \\
\hline $\mathrm{H}$ & 1.082931 & 1 & 121.882 & 1 & -180 & 1 & 2 & 1 & 5 & \# \\
\hline $\mathrm{H}$ & 1.081439 & 1 & 122.652 & 1 & 178.9747 & 1 & 8 & 7 & 6 & $\#$ \\
\hline $\mathrm{H}$ & 1.080415 & 1 & 73.85354 & 1 & -5.49056 & 1 & 11 & 3 & 2 & \# \\
\hline $\mathrm{H}$ & 1.084935 & 1 & 119.9165 & 1 & -113.187 & 1 & 12 & 11 & 3 & $\#$ \\
\hline $\mathrm{H}$ & 1.084887 & 1 & 118.7236 & 1 & 0.238757 & 1 & 17 & 13 & 12 & \# \\
\hline $\mathrm{H}$ & 1.077748 & 1 & 120.6714 & 1 & -178.76 & 1 & 18 & 17 & 13 & \# \\
\hline $\mathrm{H}$ & 1.084636 & 1 & 118.6723 & 1 & -177.652 & 1 & 25 & 24 & 23 & $\#$ \\
\hline $\mathrm{H}$ & 1.08267 & 1 & 120.2767 & 1 & -178.371 & 1 & 26 & 25 & 24 & $\#$ \\
\hline $\mathrm{H}$ & 1.079912 & 1 & 118.9094 & 1 & 179.8644 & 1 & 29 & 28 & 27 & \# \\
\hline $\mathrm{H}$ & 1.084287 & 1 & 120.0842 & 1 & -179.655 & 1 & 30 & 29 & 28 & \# \\
\hline $\mathrm{H}$ & 1.09068 & 1 & 110.8765 & 1 & 174.6839 & 1 & 31 & 3 & 2 & \# \\
\hline $\mathrm{H}$ & 1.099613 & 1 & 109.0447 & 1 & 56.06979 & 1 & 31 & 3 & 2 & $\#$ \\
\hline $\mathrm{H}$ & 1.022401 & 1 & 113.5392 & 1 & -166.041 & 1 & 34 & 19 & 18 & \# \\
\hline $\mathrm{H}$ & 1.022891 & 1 & 113.6928 & 1 & -31.7278 & 1 & 35 & 28 & 27 & \# \\
\hline $\mathrm{H}$ & 1.030045 & 1 & 114.792 & 1 & 13.42869 & 1 & 38 & 36 & 34 & \# \\
\hline $\mathrm{H}$ & 1.021347 & 1 & 114.846 & 1 & -10.3445 & 1 & 40 & 37 & 35 & $\#$ \\
\hline $\mathrm{H}$ & 1.077507 & 1 & 119.5557 & 1 & -2.3439 & 1 & 44 & 42 & 38 & \# \\
\hline $\mathrm{H}$ & 1.081597 & 1 & 118.2393 & 1 & 179.8742 & 1 & 45 & 44 & 42 & \# \\
\hline $\mathrm{H}$ & 1.081856 & 1 & 118.2709 & 1 & -179.791 & 1 & 47 & 46 & 45 & \# \\
\hline $\mathrm{H}$ & 1.084481 & 1 & 119.6292 & 1 & 179.9428 & 1 & 48 & 47 & 46 & \# \\
\hline $\mathrm{H}$ & 1.085683 & 1 & 119.7031 & 1 & -1.4373 & 1 & 49 & 43 & 40 & \# \\
\hline $\mathrm{H}$ & 1.081491 & 1 & 121.2878 & 1 & -179.986 & 1 & 50 & 49 & 43 & \# \\
\hline $\mathrm{H}$ & 1.081205 & 1 & 120.7629 & 1 & -179.975 & 1 & 52 & 51 & 50 & \# \\
\hline $\mathrm{H}$ & 1.076944 & 1 & 119.9991 & 1 & 179.133 & 1 & 53 & 52 & 51 & $\#$ \\
\hline $\mathrm{H}$ & 1.097159 & 1 & 109.986 & 1 & 75.13543 & 1 & 54 & 32 & 16 & $\#$ \\
\hline $\mathrm{H}$ & 1.09687 & 1 & 110.5262 & 1 & -45.2453 & 1 & 54 & 32 & 16 & $\#$ \\
\hline $\mathrm{H}$ & 1.092158 & 1 & 104.6633 & 1 & 165.2423 & 1 & 55 & 33 & 21 & $\#$ \\
\hline $\mathrm{H}$ & 1.095602 & 1 & 110.2698 & 1 & 48.38235 & 1 & 55 & 33 & 21 & \# \\
\hline $\mathrm{H}$ & 1.0939 & 1 & 109.7286 & 1 & -59.4381 & 1 & 56 & 54 & 32 & $\#$ \\
\hline $\mathrm{H}$ & 1.096384 & 1 & 108.329 & 1 & -177.33 & 1 & 56 & 54 & 32 & \# \\
\hline $\mathrm{H}$ & 1.094684 & 1 & 109.0959 & 1 & 49.85248 & 1 & 57 & 55 & 33 & $\#$ \\
\hline $\mathrm{H}$ & 1.094647 & 1 & 108.4219 & 1 & -66.9207 & 1 & 57 & 55 & 33 & \# \\
\hline $\mathrm{H}$ & 1.100119 & 1 & 108.8177 & 1 & 113.9036 & 1 & 58 & 7 & 6 & $\#$ \\
\hline $\mathrm{H}$ & 1.092474 & 1 & 108.4323 & 1 & -1.92789 & 1 & 58 & 7 & 6 & \# \\
\hline $\mathrm{C}$ & 2.437423 & 1 & 147.9238 & 1 & -179.112 & 1 & 51 & 50 & 49 & $\#$ \\
\hline $\mathrm{H}$ & 1.089988 & 1 & 135.0042 & 1 & -0.77384 & 1 & 93 & 51 & 50 & \# \\
\hline
\end{tabular}




\begin{tabular}{|c|c|c|c|c|c|c|c|c|c|}
\hline $\mathrm{H}$ & 1.096537 & 1 & 95.16705 & 1 & 123.9789 & 1 & 93 & 51 & 50 \\
\hline $\mathrm{H}$ & 1.09663 & 1 & 95.57926 & 1 & -126.021 & 1 & 93 & 51 & 50 \\
\hline $\mathrm{C}$ & 2.436221 & 1 & 93.33685 & 1 & -179.842 & 1 & 46 & 45 & 44 \\
\hline $\mathrm{H}$ & 1.09036 & 1 & 135.2725 & 1 & -179.865 & 1 & 97 & 46 & 45 \\
\hline $\mathrm{H}$ & 1.097007 & 1 & 95.50769 & 1 & -54.5605 & 1 & 97 & 46 & 45 \\
\hline $\mathrm{H}$ & 1.096952 & 1 & 95.19636 & 1 & 55.24312 & 1 & 97 & 46 & 45 \\
\hline $\mathrm{O}$ & 1.382923 & 1 & 115.9607 & 1 & -179.95 & 1 & 51 & 50 & 49 \\
\hline $\mathrm{O}$ & 1.387021 & 1 & 125.3348 & 1 & 179.7347 & 1 & 46 & 45 & 44 \\
\hline $\mathrm{H}$ & 1.080256 & 1 & 119.1822 & 1 & -78.3386 & 1 & 22 & 21 & 20 \\
\hline $\mathrm{H}$ & 1.080719 & 1 & 120.3791 & 1 & -178.321 & 1 & 15 & 14 & 13 \\
\hline $\mathrm{C}$ & 7.687866 & 1 & 106.9665 & 1 & -173.088 & 1 & 1 & 2 & 3 \\
\hline $\mathrm{C}$ & 1.357727 & 1 & 83.99005 & 1 & -126.181 & 1 & 105 & 1 & 2 \\
\hline $\mathrm{C}$ & 1.445235 & 1 & 115.8308 & 1 & -6.72085 & 1 & 106 & 105 & 1 \\
\hline $\mathrm{C}$ & 1.368042 & 1 & 113.4194 & 1 & -1.13903 & 1 & 107 & 106 & 105 \\
\hline S & 1.812376 & 1 & 27.41648 & 1 & 68.78008 & 1 & 105 & 1 & 2 \\
\hline $\mathrm{C}$ & 1.443291 & 1 & 131.6143 & 1 & -177.61 & 1 & 108 & 107 & 106 \\
\hline $\mathrm{C}$ & 1.374798 & 1 & 130.2136 & 1 & 64.72993 & 1 & 110 & 108 & 107 \\
\hline $\mathrm{C}$ & 1.434329 & 1 & 113.2842 & 1 & 173.5386 & 1 & 111 & 110 & 108 \\
\hline $\mathrm{C}$ & 1.368825 & 1 & 115.8327 & 1 & -0.15617 & 1 & 112 & 111 & 110 \\
\hline S & 1.816877 & 1 & 110.2505 & 1 & 0.080235 & 1 & 113 & 112 & 111 \\
\hline $\mathrm{C}$ & 6.244787 & 1 & 113.1774 & 1 & 177.7997 & 1 & 107 & 106 & 105 \\
\hline $\mathrm{C}$ & 1.375744 & 1 & 152.9639 & 1 & 115.8123 & 1 & 115 & 107 & 106 \\
\hline $\mathrm{C}$ & 1.415278 & 1 & 121.4484 & 1 & 70.54192 & 1 & 116 & 115 & 107 \\
\hline $\mathrm{C}$ & 1.436085 & 1 & 118.9037 & 1 & 1.792213 & 1 & 117 & 116 & 115 \\
\hline $\mathrm{C}$ & 1.418261 & 1 & 118.444 & 1 & -1.58729 & 1 & 118 & 117 & 116 \\
\hline $\mathrm{C}$ & 1.383739 & 1 & 121.2166 & 1 & -0.90532 & 1 & 119 & 118 & 117 \\
\hline $\mathrm{C}$ & 1.41886 & 1 & 122.3453 & 1 & -178.645 & 1 & 117 & 116 & 115 \\
\hline $\mathrm{C}$ & 1.368792 & 1 & 121.6633 & 1 & 179.784 & 1 & 121 & 117 & 116 \\
\hline $\mathrm{C}$ & 1.425906 & 1 & 120.7558 & 1 & 0.651694 & 1 & 122 & 121 & 117 \\
\hline $\mathrm{C}$ & 1.40369 & 1 & 119.3873 & 1 & 0.42134 & 1 & 123 & 122 & 121 \\
\hline $\mathrm{C}$ & 4.360237 & 1 & 120.9608 & 1 & -116.516 & 1 & 124 & 123 & 122 \\
\hline $\mathrm{C}$ & 1.380125 & 1 & 0.941809 & 1 & -23.6559 & 1 & 125 & 124 & 123 \\
\hline $\mathrm{C}$ & 1.418083 & 1 & 120.8985 & 1 & -96.1522 & 1 & 126 & 125 & 124 \\
\hline $\mathrm{C}$ & 1.435089 & 1 & 118.9215 & 1 & -0.34549 & 1 & 127 & 126 & 125 \\
\hline $\mathrm{C}$ & 1.41917 & 1 & 118.7332 & 1 & -1.01709 & 1 & 128 & 127 & 126 \\
\hline $\mathrm{C}$ & 1.372963 & 1 & 121.0579 & 1 & 1.242351 & 1 & 129 & 128 & 127 \\
\hline C & 1.435767 & 1 & 122.1355 & 1 & 177.712 & 1 & 127 & 126 & 125 \\
\hline $\mathrm{C}$ & 1.392341 & 1 & 119.3348 & 1 & -177.707 & 1 & 131 & 127 & 126 \\
\hline
\end{tabular}




\begin{tabular}{|c|c|c|c|c|c|c|c|c|c|c|}
\hline $\mathrm{C}$ & 1.418986 & 1 & 121.3151 & 1 & -0.41633 & 1 & 132 & 131 & 127 & \# \\
\hline $\mathrm{C}$ & 1.37003 & 1 & 119.9451 & 1 & -0.17961 & 1 & 133 & 132 & 131 & \# \\
\hline $\mathrm{C}$ & 1.512487 & 1 & 120.9988 & 1 & 176.5487 & 1 & 107 & 106 & 105 & \# \\
\hline $\mathrm{O}$ & 1.389741 & 1 & 116.2743 & 1 & -175.664 & 1 & 120 & 119 & 118 & \# \\
\hline $\mathrm{O}$ & 1.396549 & 1 & 117.9703 & 1 & 72.91118 & 1 & 125 & 124 & 123 & \# \\
\hline $\mathrm{N}$ & 1.397877 & 1 & 122.3691 & 1 & 176.1943 & 1 & 123 & 122 & 121 & \# \\
\hline $\mathrm{N}$ & 1.424432 & 1 & 118.2033 & 1 & 175.5161 & 1 & 132 & 131 & 127 & \# \\
\hline $\mathrm{C}$ & 1.397336 & 1 & 131.346 & 1 & 17.83041 & 1 & 138 & 123 & 122 & \# \\
\hline $\mathrm{C}$ & 1.375366 & 1 & 126.9149 & 1 & 132.9611 & 1 & 139 & 132 & 131 & \# \\
\hline $\mathrm{N}$ & 1.362937 & 1 & 110.1424 & 1 & -160.625 & 1 & 140 & 138 & 123 & \# \\
\hline$S$ & 1.727557 & 1 & 123.774 & 1 & 20.97932 & 1 & 140 & 138 & 123 & \# \\
\hline $\mathrm{N}$ & 1.35974 & 1 & 112.4898 & 1 & 170.3799 & 1 & 141 & 139 & 132 & \# \\
\hline S & 1.746449 & 1 & 121.6176 & 1 & -9.87531 & 1 & 141 & 139 & 132 & \# \\
\hline $\mathrm{C}$ & 1.418554 & 1 & 132.4348 & 1 & -171.777 & 1 & 142 & 140 & 138 & \# \\
\hline $\mathrm{C}$ & 1.42265 & 1 & 132.8209 & 1 & 172.0106 & 1 & 144 & 141 & 139 & \# \\
\hline $\mathrm{C}$ & 1.398365 & 1 & 125.8741 & 1 & 12.55919 & 1 & 146 & 142 & 140 & \# \\
\hline $\mathrm{C}$ & 1.396892 & 1 & 120.4713 & 1 & 178.2375 & 1 & 148 & 146 & 142 & \# \\
\hline $\mathrm{C}$ & 1.394726 & 1 & 121.0219 & 1 & 0.034749 & 1 & 149 & 148 & 146 & \# \\
\hline $\mathrm{C}$ & 1.402695 & 1 & 118.6872 & 1 & -0.17601 & 1 & 150 & 149 & 148 & $\#$ \\
\hline $\mathrm{C}$ & 1.38325 & 1 & 120.4653 & 1 & 0.152167 & 1 & 151 & 150 & 149 & \# \\
\hline $\mathrm{C}$ & 1.408822 & 1 & 116.1211 & 1 & 173.5782 & 1 & 147 & 144 & 141 & \# \\
\hline $\mathrm{C}$ & 1.383018 & 1 & 121.1464 & 1 & 178.7593 & 1 & 153 & 147 & 144 & \# \\
\hline $\mathrm{C}$ & 1.403091 & 1 & 120.3873 & 1 & -0.09546 & 1 & 154 & 153 & 147 & \# \\
\hline $\mathrm{C}$ & 1.395773 & 1 & 118.6816 & 1 & 0.041435 & 1 & 155 & 154 & 153 & \# \\
\hline $\mathrm{C}$ & 1.395547 & 1 & 121.0169 & 1 & 0.047973 & 1 & 156 & 155 & 154 & \# \\
\hline $\mathrm{C}$ & 1.466274 & 1 & 119.6017 & 1 & -172.527 & 1 & 136 & 120 & 119 & \# \\
\hline $\mathrm{C}$ & 1.477509 & 1 & 116.7596 & 1 & 131.3766 & 1 & 137 & 125 & 124 & \# \\
\hline $\mathrm{C}$ & 1.527986 & 1 & 106.1243 & 1 & -163.286 & 1 & 158 & 136 & 120 & \# \\
\hline $\mathrm{C}$ & 1.535564 & 1 & 111.0684 & 1 & -81.7818 & 1 & 159 & 137 & 125 & \# \\
\hline $\mathrm{C}$ & 1.516779 & 1 & 123.3296 & 1 & -5.26785 & 1 & 111 & 110 & 108 & \# \\
\hline $\mathrm{H}$ & 1.083389 & 1 & 122.0452 & 1 & 173.0009 & 1 & 106 & 105 & 1 & \# \\
\hline $\mathrm{H}$ & 1.08093 & 1 & 122.8554 & 1 & -178.302 & 1 & 112 & 111 & 110 & \# \\
\hline $\mathrm{H}$ & 1.08061 & 1 & 72.19913 & 1 & -4.52143 & 1 & 115 & 107 & 106 & \# \\
\hline $\mathrm{H}$ & 1.084978 & 1 & 119.9481 & 1 & -108.975 & 1 & 116 & 115 & 107 & \# \\
\hline $\mathrm{H}$ & 1.084905 & 1 & 118.8056 & 1 & -0.08555 & 1 & 121 & 117 & 116 & \# \\
\hline $\mathrm{H}$ & 1.07746 & 1 & 120.5426 & 1 & -178.508 & 1 & 122 & 121 & 117 & \# \\
\hline $\mathrm{H}$ & 1.084438 & 1 & 118.817 & 1 & -179.044 & 1 & 129 & 128 & 127 & \# \\
\hline $\mathrm{H}$ & 1.081967 & 1 & 119.6865 & 1 & -179.049 & 1 & 130 & 129 & 128 & \# \\
\hline
\end{tabular}




\begin{tabular}{|c|c|c|c|c|c|c|c|c|c|}
\hline $\mathrm{H}$ & 1.07982 & 1 & 119.0073 & 1 & -179.867 & 1 & 133 & 132 & 131 \\
\hline $\mathrm{H}$ & 1.084209 & 1 & 120.0934 & 1 & 179.7468 & 1 & 134 & 133 & 132 \\
\hline $\mathrm{H}$ & 1.090663 & 1 & 111.202 & 1 & 175.1214 & 1 & 135 & 107 & 106 \\
\hline $\mathrm{H}$ & 1.099675 & 1 & 109.039 & 1 & 56.64266 & 1 & 135 & 107 & 106 \\
\hline $\mathrm{H}$ & 1.022014 & 1 & 113.3669 & 1 & -167.772 & 1 & 138 & 123 & 122 \\
\hline $\mathrm{H}$ & 1.023034 & 1 & 113.4918 & 1 & -31.9768 & 1 & 139 & 132 & 131 \\
\hline $\mathrm{H}$ & 1.030366 & 1 & 114.8276 & 1 & 13.96351 & 1 & 142 & 140 & 138 \\
\hline $\mathrm{H}$ & 1.021429 & 1 & 114.8165 & 1 & -10.2466 & 1 & 144 & 141 & 139 \\
\hline $\mathrm{H}$ & 1.077367 & 1 & 119.5954 & 1 & -2.67055 & 1 & 148 & 146 & 142 \\
\hline $\mathrm{H}$ & 1.081632 & 1 & 118.226 & 1 & 179.9068 & 1 & 149 & 148 & 146 \\
\hline $\mathrm{H}$ & 1.081855 & 1 & 118.2782 & 1 & -179.804 & 1 & 151 & 150 & 149 \\
\hline $\mathrm{H}$ & 1.084436 & 1 & 119.6177 & 1 & 179.9983 & 1 & 152 & 151 & 150 \\
\hline $\mathrm{H}$ & 1.085691 & 1 & 119.7147 & 1 & -1.15898 & 1 & 153 & 147 & 144 \\
\hline $\mathrm{H}$ & 1.08148 & 1 & 121.2877 & 1 & -179.985 & 1 & 154 & 153 & 147 \\
\hline $\mathrm{H}$ & 1.081263 & 1 & 120.7637 & 1 & -179.935 & 1 & 156 & 155 & 154 \\
\hline $\mathrm{H}$ & 1.077275 & 1 & 120.1097 & 1 & 179.2779 & 1 & 157 & 156 & 155 \\
\hline $\mathrm{H}$ & 1.097189 & 1 & 109.9279 & 1 & 75.61293 & 1 & 158 & 136 & 120 \\
\hline $\mathrm{H}$ & 1.096696 & 1 & 110.7148 & 1 & -44.8709 & 1 & 158 & 136 & 120 \\
\hline $\mathrm{H}$ & 1.092362 & 1 & 104.8631 & 1 & 158.3195 & 1 & 159 & 137 & 125 \\
\hline $\mathrm{H}$ & 1.09215 & 1 & 109.9956 & 1 & 41.03939 & 1 & 159 & 137 & 125 \\
\hline $\mathrm{H}$ & 1.093896 & 1 & 109.6128 & 1 & -60.2867 & 1 & 160 & 158 & 136 \\
\hline $\mathrm{H}$ & 1.095945 & 1 & 108.4271 & 1 & -178.2 & 1 & 160 & 158 & 136 \\
\hline $\mathrm{H}$ & 1.094864 & 1 & 109.442 & 1 & 52.28691 & 1 & 161 & 159 & 137 \\
\hline $\mathrm{H}$ & 1.095056 & 1 & 108.0668 & 1 & -64.5456 & 1 & 161 & 159 & 137 \\
\hline $\mathrm{H}$ & 1.09932 & 1 & 109.4849 & 1 & 122.463 & 1 & 162 & 111 & 110 \\
\hline $\mathrm{H}$ & 1.093223 & 1 & 107.8734 & 1 & 6.202465 & 1 & 162 & 111 & 110 \\
\hline $\mathrm{C}$ & 2.437137 & 1 & 147.9156 & 1 & -179.182 & 1 & 155 & 154 & 153 \\
\hline $\mathrm{H}$ & 1.090004 & 1 & 135.0197 & 1 & -0.72674 & 1 & 197 & 155 & 154 \\
\hline $\mathrm{H}$ & 1.096549 & 1 & 95.17725 & 1 & 124.0526 & 1 & 197 & 155 & 154 \\
\hline $\mathrm{H}$ & 1.096626 & 1 & 95.54611 & 1 & -125.949 & 1 & 197 & 155 & 154 \\
\hline $\mathrm{C}$ & 2.436001 & 1 & 93.31573 & 1 & -179.851 & 1 & 150 & 149 & 148 \\
\hline $\mathrm{H}$ & 1.090387 & 1 & 135.2982 & 1 & -179.814 & 1 & 201 & 150 & 149 \\
\hline $\mathrm{H}$ & 1.097019 & 1 & 95.46082 & 1 & -54.5485 & 1 & 201 & 150 & 149 \\
\hline $\mathrm{H}$ & 1.09699 & 1 & 95.2184 & 1 & 55.24025 & 1 & 201 & 150 & 149 \\
\hline $\mathrm{O}$ & 1.382951 & 1 & 115.9456 & 1 & -179.95 & 1 & 155 & 154 & 153 \\
\hline $\mathrm{O}$ & 1.387258 & 1 & 125.323 & 1 & 179.7668 & 1 & 150 & 149 & 148 \\
\hline $\mathrm{H}$ & 1.080201 & 1 & 119.0334 & 1 & 82.69255 & 1 & 126 & 125 & 124 \\
\hline $\mathrm{H}$ & 1.080324 & 1 & 120.4013 & 1 & -178.138 & 1 & 119 & 118 & 117 \\
\hline
\end{tabular}




$\begin{array}{lrrrrrrrrrr}\mathrm{C} & 1.498329 & 1 & 146.382 & 1 & 43.67124 & 1 & 105 & 1 & 2 & \# \\ \mathrm{H} & 1.096727 & 1 & 111.2946 & 1 & -47.2992 & 1 & 209 & 105 & 1 & \# \\ \mathrm{H} & 1.096628 & 1 & 111.4065 & 1 & 73.10444 & 1 & 209 & 105 & 1 & \# \\ \mathrm{H} & 1.094808 & 1 & 109.6894 & 1 & -167.079 & 1 & 209 & 105 & 1 & \# \\ \mathrm{C} & 1.498083 & 1 & 128.833 & 1 & -179.64 & 1 & 9 & 8 & 7 & \# \\ \mathrm{H} & 1.096527 & 1 & 111.3748 & 1 & -120.874 & 1 & 213 & 9 & 8 & \# \\ \mathrm{H} & 1.094685 & 1 & 109.6009 & 1 & -1.14599 & 1 & 213 & 9 & 8 & \# \\ \mathrm{H} & 1.096803 & 1 & 111.3377 & 1 & 118.6373 & 1 & 213 & 9 & 8 & \# \\ & 0 & 0 & 0 & 0 & 0 & 0 & 0 & 0 & 0 & 0\end{array}$

Total energy $=-8123.401946$ Hartree 
the 4-mer analog with isophthalate Z-matrices

\begin{tabular}{|c|c|c|c|c|c|c|c|c|c|}
\hline $\mathrm{C}$ & 0 & 1 & 0 & 1 & 0 & 1 & 0 & 0 & 0 \\
\hline $\mathrm{C}$ & 1.444623 & 1 & 0 & 1 & 0 & 1 & 1 & 0 & 0 \\
\hline $\mathrm{C}$ & 1.34794 & 1 & 117.0334 & 1 & 0 & 1 & 2 & 1 & 0 \\
\hline $\mathrm{C}$ & 1.466866 & 1 & 112.5363 & 1 & -2.39239 & 1 & 3 & 2 & 1 \\
\hline S & 1.815938 & 1 & 110.0976 & 1 & 10.70058 & 1 & 4 & 3 & 2 \\
\hline $\mathrm{C}$ & 1.365682 & 1 & 130.3187 & 1 & -169.994 & 1 & 4 & 3 & 2 \\
\hline $\mathrm{C}$ & 1.42731 & 1 & 131.1938 & 1 & 33.50475 & 1 & 6 & 4 & 3 \\
\hline $\mathrm{C}$ & 1.399024 & 1 & 112.6663 & 1 & 179.9325 & 1 & 7 & 6 & 4 \\
\hline $\mathrm{C}$ & 1.37134 & 1 & 115.7115 & 1 & -2.89929 & 1 & 8 & 7 & 6 \\
\hline$S$ & 1.768845 & 1 & 112.1133 & 1 & -1.55098 & 1 & 9 & 8 & 7 \\
\hline $\mathrm{C}$ & 6.45143 & 1 & 116.1474 & 1 & 164.2117 & 1 & 3 & 2 & 1 \\
\hline $\mathrm{C}$ & 1.363209 & 1 & 153.7748 & 1 & 123.5658 & 1 & 11 & 3 & 2 \\
\hline $\mathrm{C}$ & 1.413804 & 1 & 121.2906 & 1 & 60.99141 & 1 & 12 & 11 & 3 \\
\hline $\mathrm{C}$ & 1.414369 & 1 & 118.9774 & 1 & 4.45311 & 1 & 13 & 12 & 11 \\
\hline $\mathrm{C}$ & 1.417807 & 1 & 118.731 & 1 & -3.24253 & 1 & 14 & 13 & 12 \\
\hline $\mathrm{C}$ & 1.366144 & 1 & 120.5081 & 1 & -1.6691 & 1 & 15 & 14 & 13 \\
\hline $\mathrm{C}$ & 1.419179 & 1 & 121.1573 & 1 & -172.647 & 1 & 13 & 12 & 11 \\
\hline $\mathrm{C}$ & 1.354163 & 1 & 120.327 & 1 & 172.5855 & 1 & 17 & 13 & 12 \\
\hline $\mathrm{C}$ & 1.4178 & 1 & 120.0344 & 1 & 3.096251 & 1 & 18 & 17 & 13 \\
\hline $\mathrm{C}$ & 1.369103 & 1 & 121.837 & 1 & 3.652159 & 1 & 19 & 18 & 17 \\
\hline $\mathrm{C}$ & 4.309164 & 1 & 111.2808 & 1 & -123.513 & 1 & 20 & 19 & 18 \\
\hline $\mathrm{C}$ & 1.361742 & 1 & 1.245666 & 1 & -28.7866 & 1 & 21 & 20 & 19 \\
\hline $\mathrm{C}$ & 1.419369 & 1 & 120.4435 & 1 & -97.6782 & 1 & 22 & 21 & 20 \\
\hline $\mathrm{C}$ & 1.413659 & 1 & 118.665 & 1 & -1.5155 & 1 & 23 & 22 & 21 \\
\hline $\mathrm{C}$ & 1.419253 & 1 & 119.0948 & 1 & -2.5493 & 1 & 24 & 23 & 22 \\
\hline $\mathrm{C}$ & 1.361764 & 1 & 121.2 & 1 & 3.716588 & 1 & 25 & 24 & 23 \\
\hline $\mathrm{C}$ & 1.435094 & 1 & 121.587 & 1 & 173.6445 & 1 & 23 & 22 & 21 \\
\hline $\mathrm{C}$ & 1.370963 & 1 & 118.3777 & 1 & -170.994 & 1 & 27 & 23 & 22 \\
\hline $\mathrm{C}$ & 1.416 & 1 & 121.7397 & 1 & -5.19341 & 1 & 28 & 27 & 23 \\
\hline $\mathrm{C}$ & 1.356512 & 1 & 120.1981 & 1 & 2.083989 & 1 & 29 & 28 & 27 \\
\hline $\mathrm{C}$ & 1.51728 & 1 & 122.5872 & 1 & 169.4865 & 1 & 3 & 2 & 1 \\
\hline $\mathrm{O}$ & 1.381674 & 1 & 116.983 & 1 & -172.7 & 1 & 16 & 15 & 14 \\
\hline $\mathrm{O}$ & 1.380863 & 1 & 117.9642 & 1 & 74.46468 & 1 & 21 & 20 & 19 \\
\hline $\mathrm{N}$ & 1.440387 & 1 & 116.56 & 1 & -175.774 & 1 & 19 & 18 & 17 \\
\hline $\mathrm{N}$ & 1.443209 & 1 & 122.2637 & 1 & 175.7358 & 1 & 28 & 27 & 23 \\
\hline $\mathrm{C}$ & 1.333055 & 1 & 123.5605 & 1 & 66.51658 & 1 & 34 & 19 & 18 \\
\hline
\end{tabular}




\begin{tabular}{|c|c|c|c|c|c|c|c|c|c|c|}
\hline $\mathrm{C}$ & 1.330644 & 1 & 124.3555 & 1 & -106.23 & 1 & 35 & 28 & 27 & \# \\
\hline $\mathrm{N}$ & 1.408904 & 1 & 112.2564 & 1 & -168.896 & 1 & 36 & 34 & 19 & \# \\
\hline S & 1.70743 & 1 & 126.4722 & 1 & 4.481402 & 1 & 36 & 34 & 19 & \# \\
\hline $\mathrm{N}$ & 1.405922 & 1 & 112.4057 & 1 & -165.388 & 1 & 37 & 35 & 28 & \# \\
\hline S & 1.70667 & 1 & 127.0937 & 1 & 7.353886 & 1 & 37 & 35 & 28 & \# \\
\hline $\mathrm{C}$ & 1.273789 & 1 & 129.6843 & 1 & -144.035 & 1 & 38 & 36 & 34 & \# \\
\hline $\mathrm{C}$ & 1.271806 & 1 & 130.3764 & 1 & -132.603 & 1 & 40 & 37 & 35 & \# \\
\hline $\mathrm{C}$ & 1.478003 & 1 & 125.906 & 1 & 10.23466 & 1 & 42 & 38 & 36 & \# \\
\hline $\mathrm{C}$ & 1.340503 & 1 & 120.6442 & 1 & -172.736 & 1 & 44 & 42 & 38 & \# \\
\hline $\mathrm{C}$ & 1.443449 & 1 & 119.8169 & 1 & -1.04877 & 1 & 45 & 44 & 42 & \# \\
\hline $\mathrm{C}$ & 1.440589 & 1 & 120.9986 & 1 & -3.65909 & 1 & 46 & 45 & 44 & \# \\
\hline $\mathrm{C}$ & 1.337206 & 1 & 119.6966 & 1 & 3.240316 & 1 & 47 & 46 & 45 & \# \\
\hline $\mathrm{C}$ & 1.472378 & 1 & 126.0839 & 1 & 7.532278 & 1 & 43 & 40 & 37 & \# \\
\hline $\mathrm{C}$ & 1.336564 & 1 & 120.2126 & 1 & -177.302 & 1 & 49 & 43 & 40 & \# \\
\hline $\mathrm{C}$ & 1.447461 & 1 & 120.4189 & 1 & -0.15416 & 1 & 50 & 49 & 43 & \# \\
\hline $\mathrm{C}$ & 1.434642 & 1 & 121.0804 & 1 & -1.86088 & 1 & 51 & 50 & 49 & \# \\
\hline $\mathrm{C}$ & 1.342621 & 1 & 119.0125 & 1 & 1.39566 & 1 & 52 & 51 & 50 & \# \\
\hline $\mathrm{C}$ & 1.434798 & 1 & 121.7532 & 1 & 178.8923 & 1 & 32 & 16 & 15 & \# \\
\hline $\mathrm{C}$ & 1.447202 & 1 & 118.909 & 1 & 121.3569 & 1 & 33 & 21 & 20 & \# \\
\hline $\mathrm{C}$ & 1.51862 & 1 & 108.3543 & 1 & -169.264 & 1 & 54 & 32 & 16 & \# \\
\hline $\mathrm{C}$ & 1.531535 & 1 & 110.6358 & 1 & -68.0697 & 1 & 55 & 33 & 21 & \# \\
\hline $\mathrm{C}$ & 1.507962 & 1 & 125.8901 & 1 & 10.68828 & 1 & 7 & 6 & 4 & \# \\
\hline $\mathrm{H}$ & 1.071584 & 1 & 120.1971 & 1 & 171.2274 & 1 & 2 & 1 & 5 & \# \\
\hline $\mathrm{H}$ & 1.067948 & 1 & 123.4161 & 1 & -178.944 & 1 & 8 & 7 & 6 & \# \\
\hline $\mathrm{H}$ & 1.069851 & 1 & 74.62818 & 1 & -3.03898 & 1 & 11 & 3 & 2 & \# \\
\hline $\mathrm{H}$ & 1.073033 & 1 & 119.7801 & 1 & -116.569 & 1 & 12 & 11 & 3 & \# \\
\hline $\mathrm{H}$ & 1.072621 & 1 & 119.2567 & 1 & -4.28302 & 1 & 17 & 13 & 12 & \# \\
\hline $\mathrm{H}$ & 1.070812 & 1 & 120.9672 & 1 & -175.865 & 1 & 18 & 17 & 13 & \# \\
\hline $\mathrm{H}$ & 1.073231 & 1 & 118.7646 & 1 & -177.723 & 1 & 25 & 24 & 23 & \# \\
\hline $\mathrm{H}$ & 1.072304 & 1 & 120.7211 & 1 & 178.4607 & 1 & 26 & 25 & 24 & \# \\
\hline $\mathrm{H}$ & 1.071375 & 1 & 118.8933 & 1 & -178.444 & 1 & 29 & 28 & 27 & \# \\
\hline $\mathrm{H}$ & 1.072687 & 1 & 120.2832 & 1 & -179.821 & 1 & 30 & 29 & 28 & \# \\
\hline $\mathrm{H}$ & 1.076064 & 1 & 111.2957 & 1 & 177.6236 & 1 & 31 & 3 & 2 & \# \\
\hline $\mathrm{H}$ & 1.085375 & 1 & 109.1053 & 1 & 59.5955 & 1 & 31 & 3 & 2 & \# \\
\hline $\mathrm{H}$ & 0.998391 & 1 & 118.5022 & 1 & -105.445 & 1 & 34 & 19 & 18 & \# \\
\hline $\mathrm{H}$ & 0.999129 & 1 & 117.4402 & 1 & 87.47337 & 1 & 35 & 28 & 27 & \# \\
\hline $\mathrm{H}$ & 1.956542 & 1 & 110.934 & 1 & 32.31139 & 1 & 38 & 36 & 34 & \# \\
\hline $\mathrm{H}$ & 2.143962 & 1 & 107.4033 & 1 & 43.75063 & 1 & 40 & 37 & 35 & \# \\
\hline
\end{tabular}




\begin{tabular}{|c|c|c|c|c|c|c|c|c|c|c|}
\hline $\mathrm{H}$ & 1.068882 & 1 & 118.806 & 1 & 7.510902 & 1 & 44 & 42 & 38 & \# \\
\hline $\mathrm{H}$ & 1.069718 & 1 & 120.6947 & 1 & 179.7211 & 1 & 45 & 44 & 42 & \# \\
\hline $\mathrm{H}$ & 1.069749 & 1 & 117.4504 & 1 & -177.5 & 1 & 47 & 46 & 45 & \# \\
\hline $\mathrm{H}$ & 1.07375 & 1 & 120.9392 & 1 & 179.1536 & 1 & 48 & 47 & 46 & \# \\
\hline $\mathrm{H}$ & 1.069421 & 1 & 118.772 & 1 & 2.120737 & 1 & 49 & 43 & 40 & \# \\
\hline $\mathrm{H}$ & 1.069672 & 1 & 122.8296 & 1 & 179.6948 & 1 & 50 & 49 & 43 & \# \\
\hline $\mathrm{H}$ & 1.069557 & 1 & 120.1557 & 1 & -178.494 & 1 & 52 & 51 & 50 & \# \\
\hline $\mathrm{H}$ & 1.073929 & 1 & 120.7932 & 1 & -179.151 & 1 & 53 & 52 & 51 & \# \\
\hline $\mathrm{H}$ & 1.082583 & 1 & 109.5592 & 1 & 70.49137 & 1 & 54 & 32 & 16 & \# \\
\hline $\mathrm{H}$ & 1.083489 & 1 & 109.9422 & 1 & -48.8067 & 1 & 54 & 32 & 16 & \# \\
\hline $\mathrm{H}$ & 1.077448 & 1 & 104.5255 & 1 & 172.1733 & 1 & 55 & 33 & 21 & \# \\
\hline $\mathrm{H}$ & 1.077985 & 1 & 109.8124 & 1 & 56.52578 & 1 & 55 & 33 & 21 & \# \\
\hline $\mathrm{H}$ & 1.083052 & 1 & 109.2677 & 1 & -56.7353 & 1 & 56 & 54 & 32 & \# \\
\hline $\mathrm{H}$ & 1.08519 & 1 & 107.3663 & 1 & -172.734 & 1 & 56 & 54 & 32 & \# \\
\hline $\mathrm{H}$ & 1.083165 & 1 & 109.5713 & 1 & 50.71124 & 1 & 57 & 55 & 33 & \# \\
\hline $\mathrm{H}$ & 1.082657 & 1 & 108.3275 & 1 & -64.8061 & 1 & 57 & 55 & 33 & \# \\
\hline $\mathrm{H}$ & 1.088296 & 1 & 105.0648 & 1 & 104.5502 & 1 & 58 & 7 & 6 & \# \\
\hline $\mathrm{H}$ & 1.076333 & 1 & 110.7571 & 1 & -8.85662 & 1 & 58 & 7 & 6 & \# \\
\hline $\mathrm{C}$ & 2.490654 & 1 & 143.228 & 1 & 178.5991 & 1 & 51 & 50 & 49 & \# \\
\hline $\mathrm{H}$ & 1.072002 & 1 & 127.4129 & 1 & -0.76591 & 1 & 93 & 51 & 50 & \# \\
\hline $\mathrm{H}$ & 1.076805 & 1 & 96.68857 & 1 & 122.757 & 1 & 93 & 51 & 50 & \# \\
\hline $\mathrm{H}$ & 1.076825 & 1 & 96.03965 & 1 & -123.653 & 1 & 93 & 51 & 50 & \# \\
\hline $\mathrm{C}$ & 2.494956 & 1 & 95.77358 & 1 & 176.4462 & 1 & 46 & 45 & 44 & \# \\
\hline $\mathrm{H}$ & 1.072133 & 1 & 127.0084 & 1 & -178.51 & 1 & 97 & 46 & 45 & \# \\
\hline $\mathrm{H}$ & 1.076879 & 1 & 95.63555 & 1 & -56.32 & 1 & 97 & 46 & 45 & \# \\
\hline $\mathrm{H}$ & 1.076841 & 1 & 97.60751 & 1 & 57.42981 & 1 & 97 & 46 & 45 & \# \\
\hline $\mathrm{O}$ & 1.28192 & 1 & 115.3336 & 1 & 178.8154 & 1 & 51 & 50 & 49 & \# \\
\hline $\mathrm{O}$ & 1.280715 & 1 & 123.2285 & 1 & 177.6773 & 1 & 46 & 45 & 44 & \# \\
\hline $\mathrm{H}$ & 1.069503 & 1 & 118.6078 & 1 & 79.52484 & 1 & 22 & 21 & 20 & \# \\
\hline $\mathrm{H}$ & 1.068891 & 1 & 120.8065 & 1 & -178.67 & 1 & 15 & 14 & 13 & \# \\
\hline $\mathrm{C}$ & 7.550113 & 1 & 105.6602 & 1 & 167.638 & 1 & 1 & 2 & 3 & \# \\
\hline $\mathrm{C}$ & 1.362331 & 1 & 82.77645 & 1 & -136.351 & 1 & 105 & 1 & 2 & \# \\
\hline $\mathrm{C}$ & 1.414342 & 1 & 115.6467 & 1 & -7.91584 & 1 & 106 & 105 & 1 & \# \\
\hline $\mathrm{C}$ & 1.397396 & 1 & 112.8826 & 1 & -1.82115 & 1 & 107 & 106 & 105 & \# \\
\hline S & 1.779167 & 1 & 29.24685 & 1 & 55.06478 & 1 & 105 & 1 & 2 & \# \\
\hline $\mathrm{C}$ & 1.392575 & 1 & 130.0418 & 1 & 179.1213 & 1 & 108 & 107 & 106 & \# \\
\hline $\mathrm{C}$ & 1.446692 & 1 & 129.7309 & 1 & 44.30997 & 1 & 110 & 108 & 107 & \# \\
\hline $\mathrm{C}$ & 1.357814 & 1 & 112.296 & 1 & -178.952 & 1 & 111 & 110 & 108 & \# \\
\hline
\end{tabular}




\begin{tabular}{|c|c|c|c|c|c|c|c|c|c|c|}
\hline $\mathrm{C}$ & 1.340057 & 1 & 128.0237 & 1 & 169.7631 & 1 & 1 & 2 & 3 & \# \\
\hline$S$ & 1.799128 & 1 & 118.9083 & 1 & -138.118 & 1 & 110 & 108 & 107 & \# \\
\hline $\mathrm{C}$ & 6.556172 & 1 & 124.2313 & 1 & 147.7705 & 1 & 107 & 106 & 105 & $\#$ \\
\hline $\mathrm{C}$ & 1.363366 & 1 & 163.4584 & 1 & 161.4005 & 1 & 115 & 107 & 106 & \# \\
\hline $\mathrm{C}$ & 1.409809 & 1 & 121.495 & 1 & 14.29437 & 1 & 116 & 115 & 107 & \# \\
\hline $\mathrm{C}$ & 1.415664 & 1 & 119.6747 & 1 & -1.62367 & 1 & 117 & 116 & 115 & \# \\
\hline $\mathrm{C}$ & 1.411423 & 1 & 117.7205 & 1 & 5.515543 & 1 & 118 & 117 & 116 & \# \\
\hline $\mathrm{C}$ & 1.373099 & 1 & 120.9071 & 1 & -5.81503 & 1 & 119 & 118 & 117 & \# \\
\hline $\mathrm{C}$ & 1.415946 & 1 & 120.0173 & 1 & 176.9254 & 1 & 117 & 116 & 115 & \# \\
\hline $\mathrm{C}$ & 1.358657 & 1 & 121.1007 & 1 & -178.002 & 1 & 121 & 117 & 116 & \# \\
\hline $\mathrm{C}$ & 1.402345 & 1 & 120.3092 & 1 & -2.73229 & 1 & 122 & 121 & 117 & \# \\
\hline $\mathrm{C}$ & 1.405503 & 1 & 121.1914 & 1 & 0.893319 & 1 & 123 & 122 & 121 & \# \\
\hline $\mathrm{C}$ & 4.307136 & 1 & 94.87509 & 1 & -131.233 & 1 & 119 & 118 & 117 & \# \\
\hline $\mathrm{C}$ & 1.369949 & 1 & 27.61534 & 1 & -25.9211 & 1 & 125 & 119 & 118 & $\#$ \\
\hline $\mathrm{C}$ & 1.40947 & 1 & 120.757 & 1 & -145.181 & 1 & 126 & 125 & 119 & \# \\
\hline $\mathrm{C}$ & 1.42914 & 1 & 118.2468 & 1 & -6.91537 & 1 & 127 & 126 & 125 & \# \\
\hline $\mathrm{C}$ & 1.389462 & 1 & 119.181 & 1 & 0.147775 & 1 & 128 & 127 & 126 & \# \\
\hline $\mathrm{C}$ & 1.382159 & 1 & 121.0325 & 1 & 4.409945 & 1 & 129 & 128 & 127 & \# \\
\hline $\mathrm{C}$ & 1.428241 & 1 & 123.6644 & 1 & 177.9401 & 1 & 127 & 126 & 125 & \# \\
\hline $\mathrm{C}$ & 1.504752 & 1 & 114.3113 & 1 & -161.437 & 1 & 131 & 127 & 126 & \# \\
\hline $\mathrm{C}$ & 1.461467 & 1 & 118.4227 & 1 & -34.8862 & 1 & 132 & 131 & 127 & \# \\
\hline $\mathrm{C}$ & 1.326305 & 1 & 118.6349 & 1 & 23.35703 & 1 & 133 & 132 & 131 & \# \\
\hline $\mathrm{C}$ & 1.504297 & 1 & 120.9084 & 1 & 167.3505 & 1 & 107 & 106 & 105 & \# \\
\hline $\mathrm{O}$ & 1.378782 & 1 & 116.9736 & 1 & -177.895 & 1 & 120 & 119 & 118 & \# \\
\hline $\mathrm{O}$ & 1.381487 & 1 & 93.13327 & 1 & 132.4484 & 1 & 125 & 119 & 118 & \# \\
\hline $\mathrm{N}$ & 1.419077 & 1 & 116.7784 & 1 & -175.887 & 1 & 123 & 122 & 121 & \# \\
\hline $\mathrm{N}$ & 1.263142 & 1 & 122.5565 & 1 & 135.6212 & 1 & 132 & 131 & 127 & \# \\
\hline $\mathrm{C}$ & 1.376197 & 1 & 122.8864 & 1 & 84.30229 & 1 & 138 & 123 & 122 & \# \\
\hline $\mathrm{C}$ & 1.380358 & 1 & 135.4501 & 1 & -3.20665 & 1 & 139 & 132 & 131 & \# \\
\hline $\mathrm{N}$ & 1.321574 & 1 & 114.0629 & 1 & -165.664 & 1 & 140 & 138 & 123 & \# \\
\hline$S$ & 1.749178 & 1 & 119.8813 & 1 & 16.12397 & 1 & 140 & 138 & 123 & \# \\
\hline $\mathrm{N}$ & 1.311695 & 1 & 113.388 & 1 & 111.8179 & 1 & 141 & 139 & 132 & \# \\
\hline$S$ & 1.75611 & 1 & 116.8337 & 1 & -75.2125 & 1 & 141 & 139 & 132 & \# \\
\hline $\mathrm{C}$ & 1.430321 & 1 & 127.7178 & 1 & -176.727 & 1 & 142 & 140 & 138 & \# \\
\hline $\mathrm{C}$ & 1.423429 & 1 & 132.3176 & 1 & 171.9713 & 1 & 144 & 141 & 139 & \# \\
\hline $\mathrm{C}$ & 1.382093 & 1 & 122.5357 & 1 & -56.384 & 1 & 146 & 142 & 140 & \# \\
\hline $\mathrm{C}$ & 1.389209 & 1 & 120.2823 & 1 & -177.459 & 1 & 148 & 146 & 142 & \# \\
\hline $\mathrm{C}$ & 1.386591 & 1 & 119.8878 & 1 & 0.83968 & 1 & 149 & 148 & 146 & \# \\
\hline
\end{tabular}




\begin{tabular}{|c|c|c|c|c|c|c|c|c|c|c|}
\hline $\mathrm{C}$ & 1.391169 & 1 & 119.9196 & 1 & 0.15479 & 1 & 150 & 149 & 148 & $\#$ \\
\hline $\mathrm{C}$ & 1.378978 & 1 & 119.8767 & 1 & -0.67172 & 1 & 151 & 150 & 149 & $\#$ \\
\hline $\mathrm{C}$ & 1.384063 & 1 & 125.7366 & 1 & 20.94786 & 1 & 147 & 144 & 141 & \# \\
\hline $\mathrm{C}$ & 1.393 & 1 & 120.0932 & 1 & 179.3415 & 1 & 153 & 147 & 144 & $\#$ \\
\hline $\mathrm{C}$ & 1.384011 & 1 & 120.4891 & 1 & -0.36591 & 1 & 154 & 153 & 147 & $\#$ \\
\hline $\mathrm{C}$ & 1.393028 & 1 & 119.5434 & 1 & -0.83543 & 1 & 155 & 154 & 153 & $\#$ \\
\hline $\mathrm{C}$ & 1.377126 & 1 & 120.0571 & 1 & 0.665356 & 1 & 156 & 155 & 154 & \# \\
\hline $\mathrm{C}$ & 1.441427 & 1 & 121.0629 & 1 & -178.772 & 1 & 136 & 120 & 119 & $\#$ \\
\hline $\mathrm{C}$ & 1.449778 & 1 & 117.9599 & 1 & 93.11056 & 1 & 137 & 125 & 119 & $\#$ \\
\hline $\mathrm{C}$ & 1.516504 & 1 & 108.0538 & 1 & 172.4501 & 1 & 158 & 136 & 120 & $\#$ \\
\hline $\mathrm{C}$ & 1.52852 & 1 & 111.3059 & 1 & -55.5575 & 1 & 159 & 137 & 125 & \# \\
\hline $\mathrm{C}$ & 1.520157 & 1 & 124.8228 & 1 & 9.496954 & 1 & 111 & 110 & 108 & $\#$ \\
\hline $\mathrm{H}$ & 1.070502 & 1 & 121.9635 & 1 & 171.5926 & 1 & 106 & 105 & 1 & \# \\
\hline $\mathrm{H}$ & 1.07125 & 1 & 122.5003 & 1 & -178.12 & 1 & 112 & 111 & 110 & $\#$ \\
\hline $\mathrm{H}$ & 1.068829 & 1 & 76.32023 & 1 & -4.74366 & 1 & 115 & 107 & 106 & \# \\
\hline $\mathrm{H}$ & 1.072527 & 1 & 119.7627 & 1 & -165.669 & 1 & 116 & 115 & 107 & $\#$ \\
\hline $\mathrm{H}$ & 1.072701 & 1 & 118.8399 & 1 & 1.875632 & 1 & 121 & 117 & 116 & \# \\
\hline $\mathrm{H}$ & 1.069681 & 1 & 121.468 & 1 & -179.046 & 1 & 122 & 121 & 117 & $\#$ \\
\hline $\mathrm{H}$ & 1.071284 & 1 & 119.2991 & 1 & -175.833 & 1 & 129 & 128 & 127 & \# \\
\hline $\mathrm{H}$ & 1.072304 & 1 & 120.5959 & 1 & 178.4845 & 1 & 130 & 129 & 128 & $\#$ \\
\hline $\mathrm{H}$ & 1.069278 & 1 & 117.2353 & 1 & -159.805 & 1 & 133 & 132 & 131 & \# \\
\hline $\mathrm{H}$ & 1.071113 & 1 & 121.5756 & 1 & 178.4546 & 1 & 134 & 133 & 132 & $\#$ \\
\hline $\mathrm{H}$ & 1.072863 & 1 & 111.9817 & 1 & 162.513 & 1 & 135 & 107 & 106 & \# \\
\hline $\mathrm{H}$ & 1.083882 & 1 & 109.4328 & 1 & 42.76354 & 1 & 135 & 107 & 106 & $\#$ \\
\hline $\mathrm{H}$ & 1.014476 & 1 & 114.7341 & 1 & -77.0373 & 1 & 138 & 123 & 122 & \# \\
\hline $\mathrm{H}$ & 3.007291 & 1 & 97.87828 & 1 & 99.83515 & 1 & 157 & 156 & 155 & $\#$ \\
\hline $\mathrm{H}$ & 1.012098 & 1 & 118.0934 & 1 & -0.34233 & 1 & 142 & 140 & 138 & $\#$ \\
\hline $\mathrm{H}$ & 1.023653 & 1 & 114.1355 & 1 & -1.60802 & 1 & 144 & 141 & 139 & \# \\
\hline $\mathrm{H}$ & 1.069636 & 1 & 120.1237 & 1 & 3.234616 & 1 & 148 & 146 & 142 & $\#$ \\
\hline $\mathrm{H}$ & 1.070161 & 1 & 119.0678 & 1 & -179.51 & 1 & 149 & 148 & 146 & \# \\
\hline $\mathrm{H}$ & 1.070663 & 1 & 118.5169 & 1 & 179.1244 & 1 & 151 & 150 & 149 & $\#$ \\
\hline $\mathrm{H}$ & 1.072847 & 1 & 120.106 & 1 & 179.1176 & 1 & 152 & 151 & 150 & \# \\
\hline $\mathrm{H}$ & 1.066309 & 1 & 120.6196 & 1 & -0.46153 & 1 & 153 & 147 & 144 & $\#$ \\
\hline $\mathrm{H}$ & 1.070787 & 1 & 118.7131 & 1 & -179.784 & 1 & 154 & 153 & 147 & \# \\
\hline $\mathrm{H}$ & 1.070819 & 1 & 118.6243 & 1 & -177.702 & 1 & 156 & 155 & 154 & $\#$ \\
\hline $\mathrm{H}$ & 1.073379 & 1 & 119.8219 & 1 & -176.507 & 1 & 157 & 156 & 155 & \# \\
\hline $\mathrm{H}$ & 1.081455 & 1 & 109.6264 & 1 & 53.43186 & 1 & 158 & 136 & 120 & $\#$ \\
\hline $\mathrm{H}$ & 1.082896 & 1 & 109.4299 & 1 & -65.8694 & 1 & 158 & 136 & 120 & \# \\
\hline
\end{tabular}




\begin{tabular}{|c|c|c|c|c|c|c|c|c|c|c|}
\hline $\mathrm{H}$ & 1.077444 & 1 & 104.0766 & 1 & -176.578 & 1 & 159 & 137 & 125 & $\#$ \\
\hline $\mathrm{H}$ & 1.083689 & 1 & 109.3265 & 1 & 67.57506 & 1 & 159 & 137 & 125 & $\#$ \\
\hline $\mathrm{H}$ & 1.083351 & 1 & 108.4703 & 1 & -58.3843 & 1 & 160 & 158 & 136 & \# \\
\hline $\mathrm{H}$ & 1.084429 & 1 & 108.1917 & 1 & -174.582 & 1 & 160 & 158 & 136 & $\#$ \\
\hline $\mathrm{H}$ & 1.083028 & 1 & 108.7576 & 1 & 62.80891 & 1 & 161 & 159 & 137 & $\#$ \\
\hline $\mathrm{H}$ & 1.082134 & 1 & 108.9863 & 1 & -52.9033 & 1 & 161 & 159 & 137 & $\#$ \\
\hline $\mathrm{H}$ & 1.08672 & 1 & 107.0702 & 1 & 108.9031 & 1 & 162 & 111 & 110 & \# \\
\hline $\mathrm{H}$ & 1.079056 & 1 & 110.3944 & 1 & -5.67298 & 1 & 162 & 111 & 110 & $\#$ \\
\hline $\mathrm{C}$ & 2.450216 & 1 & 95.24478 & 1 & -176.125 & 1 & 155 & 154 & 153 & $\#$ \\
\hline $\mathrm{H}$ & 1.075213 & 1 & 133.3921 & 1 & -178.844 & 1 & 197 & 155 & 154 & $\#$ \\
\hline $\mathrm{H}$ & 1.082079 & 1 & 97.94009 & 1 & -51.9322 & 1 & 197 & 155 & 154 & \# \\
\hline $\mathrm{H}$ & 1.081694 & 1 & 94.00609 & 1 & 58.94557 & 1 & 197 & 155 & 154 & $\#$ \\
\hline $\mathrm{C}$ & 2.448682 & 1 & 94.53577 & 1 & 179.1533 & 1 & 150 & 149 & 148 & \# \\
\hline $\mathrm{H}$ & 1.075452 & 1 & 133.6618 & 1 & 179.6892 & 1 & 201 & 150 & 149 & $\#$ \\
\hline $\mathrm{H}$ & 1.081679 & 1 & 95.56718 & 1 & -56.0946 & 1 & 201 & 150 & 149 & \# \\
\hline $\mathrm{H}$ & 1.081727 & 1 & 96.14923 & 1 & 54.74389 & 1 & 201 & 150 & 149 & $\#$ \\
\hline $\mathrm{O}$ & 1.364638 & 1 & 124.5729 & 1 & 179.2389 & 1 & 155 & 154 & 153 & \# \\
\hline $\mathrm{O}$ & 1.366294 & 1 & 124.2719 & 1 & 179.931 & 1 & 150 & 149 & 148 & $\#$ \\
\hline $\mathrm{H}$ & 1.064321 & 1 & 117.7142 & 1 & 31.78497 & 1 & 126 & 125 & 119 & \# \\
\hline $\mathrm{H}$ & 1.068733 & 1 & 120.9343 & 1 & 171.9279 & 1 & 119 & 118 & 117 & $\#$ \\
\hline $\mathrm{C}$ & 1.489987 & 1 & 149.0589 & 1 & -139.974 & 1 & 105 & 1 & 113 & \# \\
\hline $\mathrm{H}$ & 1.083256 & 1 & 111.2426 & 1 & -48.0388 & 1 & 209 & 105 & 1 & $\#$ \\
\hline $\mathrm{H}$ & 1.083999 & 1 & 110.8171 & 1 & 71.69745 & 1 & 209 & 105 & 1 & \# \\
\hline $\mathrm{H}$ & 1.08042 & 1 & 110.2959 & 1 & -168.426 & 1 & 209 & 105 & 1 & \# \\
\hline $\mathrm{C}$ & 1.48758 & 1 & 126.6136 & 1 & 178.6207 & 1 & 9 & 8 & 7 & \# \\
\hline $\mathrm{H}$ & 1.085456 & 1 & 110.1768 & 1 & -109.019 & 1 & 213 & 9 & 8 & $\#$ \\
\hline $\mathrm{H}$ & 1.080172 & 1 & 109.9976 & 1 & 10.84981 & 1 & 213 & 9 & 8 & $\#$ \\
\hline $\mathrm{H}$ & 1.082417 & 1 & 111.4626 & 1 & 131.6826 & 1 & 213 & 9 & 8 & \# \\
\hline $\mathrm{C}$ & 5.524145 & 1 & 115.2601 & 1 & 62.13421 & 1 & 213 & 9 & 8 & $\#$ \\
\hline $\mathrm{C}$ & 1.389957 & 1 & 101.038 & 1 & 62.52342 & 1 & 217 & 213 & 9 & \# \\
\hline $\mathrm{C}$ & 1.390315 & 1 & 120.4011 & 1 & -52.1659 & 1 & 218 & 217 & 213 & $\#$ \\
\hline $\mathrm{C}$ & 1.384064 & 1 & 119.351 & 1 & 0.136629 & 1 & 219 & 218 & 217 & \# \\
\hline $\mathrm{C}$ & 1.39088 & 1 & 120.5902 & 1 & 1.17341 & 1 & 220 & 219 & 218 & $\#$ \\
\hline $\mathrm{C}$ & 1.383778 & 1 & 50.57545 & 1 & -57.5395 & 1 & 217 & 213 & 9 & $\#$ \\
\hline $\mathrm{H}$ & 1.071527 & 1 & 115.8689 & 1 & -166.215 & 1 & 217 & 213 & 9 & $\#$ \\
\hline $\mathrm{H}$ & 1.071295 & 1 & 120.9657 & 1 & 127.3006 & 1 & 218 & 217 & 213 & \# \\
\hline $\mathrm{H}$ & 1.070018 & 1 & 118.164 & 1 & 177.7651 & 1 & 220 & 219 & 218 & $\#$ \\
\hline $\mathrm{H}$ & 1.07192 & 1 & 120.6727 & 1 & -99.3922 & 1 & 222 & 217 & 213 & \# \\
\hline
\end{tabular}




$\begin{array}{rrrrrrrrrrr}\mathrm{C} & 1.471951 & 1 & 120.7206 & 1 & 175.1347 & 1 & 221 & 220 & 219 & \# \\ \mathrm{O} & 1.220062 & 1 & 124.25 & 1 & -150.884 & 1 & 227 & 221 & 220 & \# \\ \mathrm{C} & 1.500545 & 1 & 120.4378 & 1 & 177.1333 & 1 & 219 & 218 & 217 & \# \\ \mathrm{O} & 1.245534 & 1 & 117.9815 & 1 & -17.5851 & 1 & 229 & 219 & 218 & \# \\ \mathrm{O} & 1.355494 & 1 & 118.278 & 1 & 26.01854 & 1 & 227 & 221 & 220 & \# \\ \mathrm{O} & 1.281205 & 1 & 117.7252 & 1 & 164.3376 & 1 & 229 & 219 & 218 & \# \\ \mathrm{C} & 5.123996 & 1 & 113.0307 & 1 & 21.55164 & 1 & 38 & 36 & 34 & \# \\ \mathrm{C} & 1.392769 & 1 & 146.5407 & 1 & 146.2931 & 1 & 233 & 38 & 36 & \# \\ \mathrm{C} & 1.388435 & 1 & 119.7709 & 1 & -177.688 & 1 & 234 & 233 & 38 & \# \\ \mathrm{C} & 1.387745 & 1 & 120.2448 & 1 & -0.36915 & 1 & 235 & 234 & 233 & \# \\ \mathrm{C} & 1.393254 & 1 & 119.7434 & 1 & -1.24777 & 1 & 236 & 235 & 234 & \# \\ \mathrm{C} & 1.383222 & 1 & 93.59438 & 1 & -33.2416 & 1 & 233 & 38 & 36 & \# \\ \mathrm{H} & 1.072146 & 1 & 119.6506 & 1 & 1.577944 & 1 & 234 & 233 & 38 & \# \\ \mathrm{H} & 1.070792 & 1 & 119.8675 & 1 & 179.3985 & 1 & 235 & 234 & 233 & \# \\ \mathrm{H} & 1.072148 & 1 & 120.5425 & 1 & 179.3762 & 1 & 236 & 235 & 234 & \# \\ \mathrm{H} & 1.064696 & 1 & 119.6504 & 1 & -1.57822 & 1 & 238 & 233 & 38 & \# \\ \mathrm{C} & 1.466236 & 1 & 25.2157 & 1 & 146.6576 & 1 & 233 & 38 & 36 & \# \\ \mathrm{O} & 1.216872 & 1 & 126.8335 & 1 & 166.907 & 1 & 243 & 233 & 38 & \# \\ \mathrm{C} & 1.464978 & 1 & 121.3994 & 1 & 179.8897 & 1 & 237 & 236 & 235 & \# \\ \mathrm{O} & 1.217959 & 1 & 126.9257 & 1 & -16.6853 & 1 & 245 & 237 & 236 & \# \\ \mathrm{O} & 1.356488 & 1 & 112.8827 & 1 & 165.475 & 1 & 245 & 237 & 236 & \# \\ \mathrm{O} & 1.3553 & 1 & 112.8888 & 1 & -12.7674 & 1 & 243 & 233 & 38 & \# \\ & 0 & 0 & 0 & 0 & 0 & 0 & 0 & 0 & 0 & 0\end{array}$

Total energy $=-9334.987231$ Hartree 


\section{Comparison ${ }^{1} \mathrm{H}$ NMR Spectra}

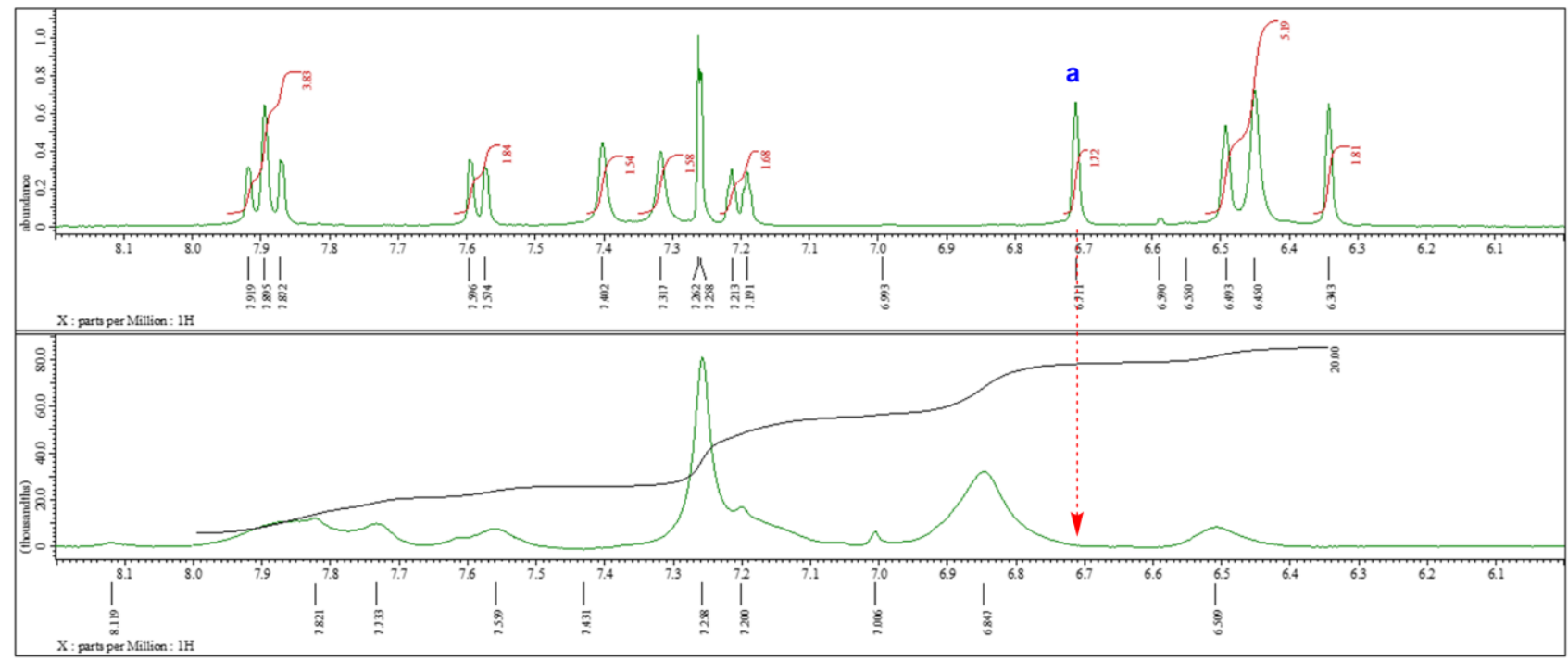

$\delta / \mathrm{ppm}$

Figure S46. Enlarged ${ }^{1} \mathrm{H}$ NMR spectra (400 MHz) of $\mathbf{M}$ (top) and $\mathbf{P}$ (bottom).

Discussion: The reactive $\alpha$ proton of the bithiophene monomer in $\mathbf{M}$ completely disappeared in $\mathbf{P}$, indicating that the polymerization occurred efficiently without some oligomers, especially dimers, as main products. 